\author{
Universidade de São Paulo \\ Instituto de Física
}

\title{
Montagem de uma pinça óptica e sua \\ calibração: estudo teórico e simulação computacional de sua força
}

Ricardo Rovere de Santi

Orientador: Prof. Dr. Adriano Mesquita Alencar

Versão corrigida

A versão original se encontra disponível na bliblioteca do instituto de fisca

Dissertação de mestrado apresentada ao Instituto de Física como requisito parcial para a obtenção do título de Mestre em Ciências.

Banca Examinadora:

Prof. Dr. Adriano Mequita Alencar (IF-USP)

Prof. Dr. Henrique Takachi Moriya (EPUSP)

Prof. Dra . Isis Vasconcelos de Brito(Senac)

São Paulo 


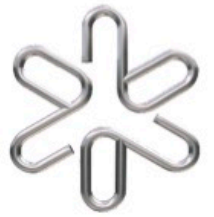

UNIVERSIDADE DE SÃO PAULO

INSTITUTO DE FÍSICA

PROGRAMA DE PÓS-GRADUAÇÃO EM FÍSICA

\section{Montagem de uma pinça óptica e calibração: estudo teórico e simulação computacional de sua força}

\author{
Dissertação de Mestrado
}

\section{Ricardo Rovere de Santi}

\author{
Adumol/tancer. \\ Orientador: Prof. Dr. Adriano Mesquita Alencar \\ Laboratório de Microrreologia e Fisiologia Molecular \\ Departamento de Física Geral \\ Instituto de Física - Universidade de São Paulo
}


Dedico essa obra aos meus pais e minha família que sempre me amaram e me apoiaram. 


\section{Agradecimentos}

Eu gostaria de agradecer as pessoas que tornaram esse trabalho possível, 1-Primeiro meus pais e famílias por sempre acreditarem em mim, e me apoiarem.

2- Minha Dra. Isis Vaconceslos de Brito, por me ajudar a construir a pinça óptica a qual não poderia ter feito sem ela, além de me ajudar diversas vezes durante meu mestrado, a qual eu não poderia estar concluído sem a ajuda dela.

3- Meu Orientador Adriano Mesquita Alencar por me orientar durante todo o processo apesar de estar bem ocupado durante meu mestrado.

4-Ao técnico do laboratório Antonio Carlos Bloise Junio, o técnico do laboratório, LabM², a qual eu faço parte, e por me ajudar em diversas situações durante a montagem da pinça óptica, principalmente por criar o Microcontrolador que utilizei para fazer a calibração da pinça óptica.

5- A Prof. ${ }^{a}$ Ligia Ferreira Gomes, membro do laboratório $\mathrm{LabM}^{2}$, por me ajudar durante todo o processo e por sua ajuda durante uma um problema familiar que tive, além de indicar a Camila a pessoa que revisou o português da minha dissertação.

6- A estudante de doutorado e membros do laboratório LabM² ,Jennifer Adriane dos Santos, por me ajudar em diversas vezes durante meu mestrado, me ajudando com na montagem de algumas figuras.

7-A estudante de doutorado Adriana Valério, ex-membro do laboratório, por me ajudar nas disciplinas do mestrado alem de me ajudar a operar o programa que usei na calibração da pinça óptica apressar de dela estar muito ocupada.

8-Ao estudante de doutorando Yan Borges Barreto, membro do laboratório LabM², que me ajudou em algumas disciplinas da pós-graduação, e no exame de proficiência em inglês.

9- Ao pós doutorando, Dr. Alexandre Barros de Almeida, por me ajudar durante alguns problemas que tive com computador do laboratório, e com alguns de meus programas.

10-Ao professor doutor Antonio Álvaro Rainha Neves da UFABC, por revisar partes da minha dissertação me ajudar a entender a teoria da força óptica e os probelams com o experimento, além de ter me ajudar muito no desenvolvimento dos programas da simulações das forças que não teria conseguido concluir sem ajuda dele .

11-Ao professor Doutor Carlos Lenz Cesar da Unicamp, por me ajudar a conseguir fazer pinça óptica funcionar, quando nós estávamos empacadas na sua construção. Alem de me ajudar com entendimento de vários aspectos do funcionamento experimental da pinça óptica. 
12- Ao Professor Doutor Vito Vanin da USP, por me ajudar durante o desenvolvimento dos programas utilizados na calibração da pinça óptica.

13-Aos funcionários da CPG por terem me ajudado com burocracia do projeto de mestrado e pela prorrogação do prazo do projeto.

14- Ao técnico de informática, José Valdir Spadacini, por me ajudar com alguns problemas técnicos.

15-Ao estudante de doutorado Arthur Prado Camargo, membros do $\mathrm{LabM}^{2}$, por me ajudar com confecção de alguns documentos e textos.

16- Aos membros da secretaria de física geral, pela ajuda durante a minha estada na USP.

17- Por revisar o português da minha dissertação a aluna de doutorado Camila Machado da escola de psicologia.

18-A Olenca dona do da casa do airb, que facilitou minha estadia em São Paulo em quanto eu trabalhava na pinça óptica.

19- A Capes por providenciar uma bolsa temporária para mim, e FAPESP por financiar o laboratório $\mathrm{LabM}^{2} \mathrm{e}$ seus equipamentos.

20- E por Fim todos os funcionários da USP, que fazem funcionar essa que é a maior universidade do Brasil. 
Não há no intelecto que não esteja primeiro nos sentidos, com exceção do próprio intelecto.

Gottfried Wilhelm Leibniz 


\section{Resumo}

A pinça óptica tem se tornado uma importante ferramenta na análise de sistemas biológicos, podendo ser utilizada, desde o estudo de moléculas de DNA, ao estudo de microorganismos, permitindo que sejam medidas as suas propriedades físicas e reológicas, ou manipulando sua posição para estudar seu comportamento. O presente projeto de pesquisa consistiu em montar um aparato de pinça óptica para aplicar em técnicas microscópicas, tais como microrreologia. Estudamos o fundamento teórico da pinça e realizamos a análise numérica através de simulações da força no regime de Lorenz - Mie. Esse trabalho permitirá a adaptação de uma pinça óptica ao sistema de Microscopia de Força e Tração (TFM), para o estudo mais amplo de propriedades mecânicas de células vivas.

Palavras-Chaves: Pinça óptica, GLMT,simulação computacional de força óptica no regime de Lorenz - Mie 


\section{Abstract}

Optical tweezers have become an important tool in the analysis of biological systems, and can be used from the study of DNA and molecules to the study of microorganisms, allowing their physical and rheological properties to be measured, or manipulating their position to study their behavior. The present research project consisted of mounting an optical tweezers apparatus to apply on microscopic techniques, such as microrheology. It was intended to study the theoretical basis of the tweezers and to perform the numerical analysis through simulations of the force under the Lorenz-Mie regime. This work will allow the construction of an optical tweezers coupled to Traction Force Microscopy (TFM) in the future, for the broader study of mechanical properties of living cells.

Keywords: optical tweezer, GLMT, Computer simulation of optical force in the Lorenz - Mie regime 


\section{Sumário}

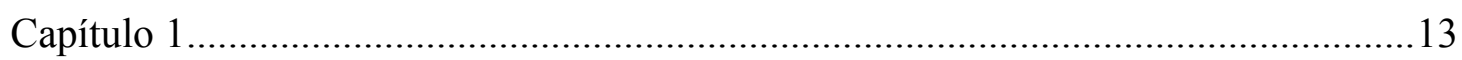

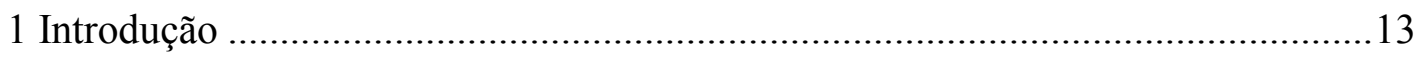

1.1 Introdução do conceito de pressão de radiação ..............................................13

1.2 Algumas aplicações da pinça óptica........................................................... 14

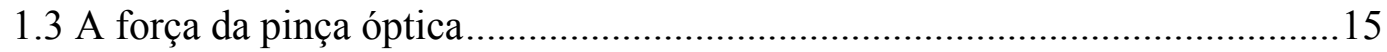

1.4 Construção de pinça óptica e calibração e comparação numérica usando a

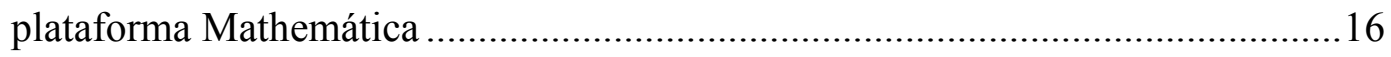

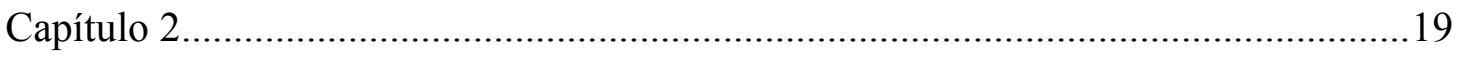

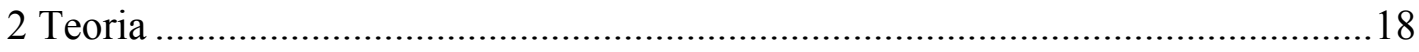

2.1 Introdução à teoria da força da pinça óptica................................................. 19

2.2 Teoria da força em regime optico ou deRayleigh .......................................25

2.3 Teoria da força para regime optico...........................................................25

2.4 Teorias eletromagnéticas de (Lorenz - Mie) para .........................................34

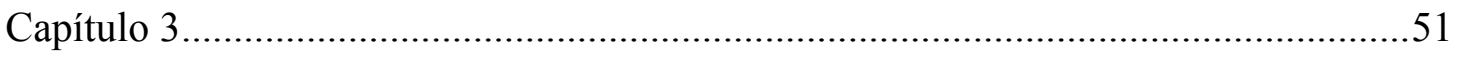

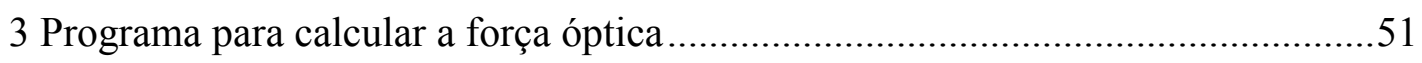

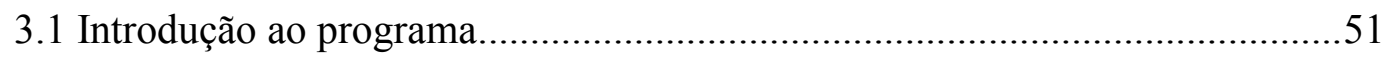

3.2 Simulação da força axial sem deslocamento focal ........................................51

3.3 Simulação da força axial com deslocamento focal .........................................53

3.4 Cálculos da constante elástica $\mathrm{k}$ a partir da força simulada axial ...................55

3.5 Simulações da força radial sem deslocamento focal ........................................55

3.6 Simulações da força radial com deslocamento focal.......................................58

3.7 Cálculo da constante elástica $\mathrm{k}$ a partir da força simulada Radial ..................59

3.8 O programa utilizado na análise dos dados do movimento Browniano ..........60

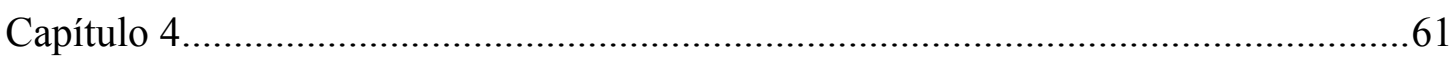

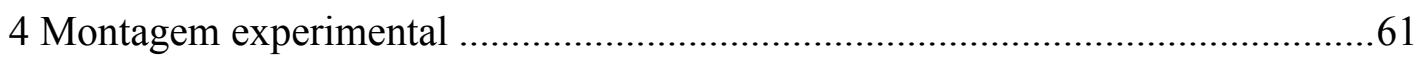


4.1 O esquema da pinça

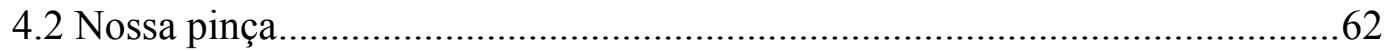

4.3 Método de montagem e problemas que podem ocorrer .................................66

4.4 Calibrações em x, y ou calibração radial.........................................................69

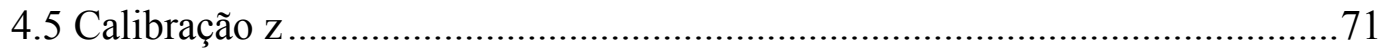

4.6 Representação em matriz dos componentes ópticos da pinça .........................71

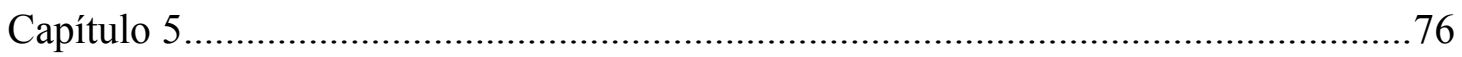

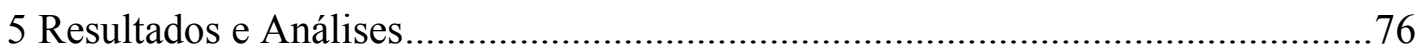

5.1 Resultado do experimento de máxima velocidade para força radial...............76

5.2. Resultado da simulação da força para o caso axial .......................................79

5.3 Resultados do experimento do movimento Browniano .................................81

5.4 Resultados experimentais do experimento de força viscosa comparada com as simulações do

5.5 Resultado das simulações para ou axial com e sem distância focal, com o deslocamento focal igual ao caso experimental radial

5.6 Discussão sobre as dificuldades de simular as forças ópticas no regime de Mie

Capítulo 6 .99

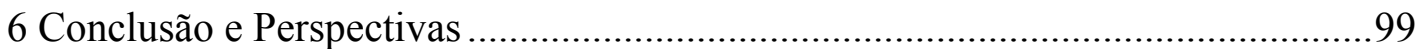

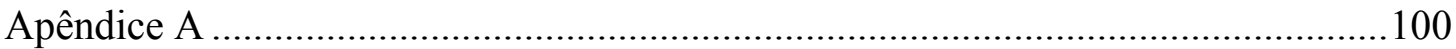

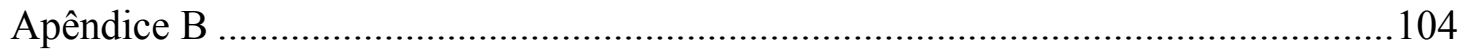

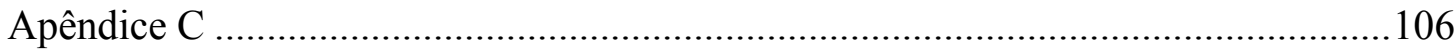

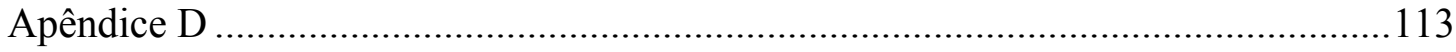

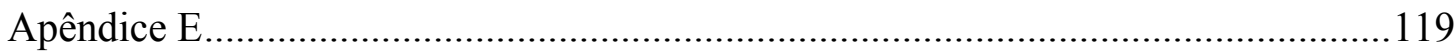

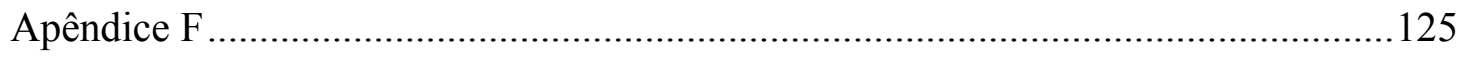

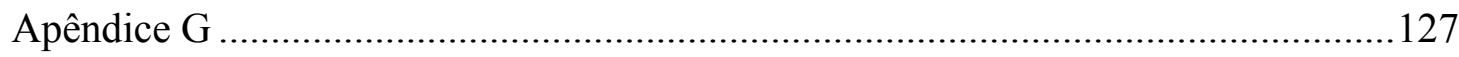




\section{Índice de Figuras}

Figura 2.1: Representação do momento do feixe que, ao ser refratado pela partícula, exerce uma força e sofre recuo ao transferir momento para ela

Figura 2.2: Raios refratados para uma força dominante gradiente

Figura 2.3: Raios refratados resudando uma força dominante de esplhamento.

Figura 2.4: Diagramas das forças de espalhamento e sua direçao

Figura 2.5: Diagramas da força optica ao refletir e refratar infinitas vezes na dentro da microesfera.

Figura 2.6: representaçao de um raio de luz refratado numa esfera sem aberraçao esferica. 26

Figura 2.7: Desenho ilustrado o fenômeno de aberaçâo esferica.

Figura 2.8: representaçao de um raio de luz refratado numa esfera com aberraçao

esferica.

Figura 2.9:Reprentaçao da onda eltromgnetica atravessando oleo e o vidro, e atigindo a as particulas na amostra.

Figura 2.10: Diagrama da mudaçao de cordenadas para cordenads esfericas para cilindricas da onda eletromagnetica.

Figura 4.1(a)Diagramas do percusso do laser dentro do mcroscopio(b)e(c) fotos da pinça otica.

Figura 4.2: Primeira foto da pinça otica com legendas para seus componentes.

Figura 4.3: Segunda fotos da pinça otica com legendas para seus componentes.

Figura 4.4: Fotos do hardowino liguado ao motor de passo

Figura 4.5: Fotos da pinça otica com legendas para seus

componentes. 
Figura 4.6: Fotos de uma microesferas pressa pela armadilha optica com as reflexões do laser.

Figura 4.7: Diagrama do percusso do

laser.

Figura 5.1(a): Foto do painel de controle do programa

Tracker. 76

Figura B.1: Diagrama para deduçao do ângulo maximo de refraçao .98

Figura 2.1, 2.22 .3 e 2.10 retiradas e adaptadas de A. A. R. Neves. Força óptica em pinças ópticas: estudo teórico e experimental. 2006. 169 p. Tese (doutorado) - Universidade Estadual de Campinas, Instituto de Física Gleb Wataghin, Campinas, SP.

Figura 2.6 e 2.8 e B.1 foram retiradas e adaptadas de P. S. Alves. Teoria de calibração de pinça óptica. 2012. 74 p. Dissertação (mestrado) - Universidade Federal de Viçosa, Viçosa, MG.

A Figura 2.5 foi retirada e adaptada de Ashkin, A. (1992). Forces of a single-beam gradient laser trap on a dielectric sphere in the ray optics regime. Biophysical Journal, 61(2), 569-582. 


\section{Capítulo 1}

\section{Introdução}

\subsection{Introdução do conceito de pressão de radiação}

O aprisionamento óptico é baseado nas teorias de pressão de radiação que foram observadas e propostas, pela primeira vez, pelo astrônomo alemão Johannes Kepler, que, em 1609, observou que a cauda dos cometas estava sempre na direção contrária ao Sol. Além de propor esse fenômeno, Kepler também propôs uma aplicação tecnológica na qual seria possível viajar ao espaço ou da Terra à Lua usado esse princípio, que, atualmente, se tornou realidade com o advento das velas solares. Porém, apenas em meados do século XIX, foi desenvolvido um modelo com a descrição da luz como uma onda eletromagnética, por Helmholtz Maxwell. Em 1873, Maxwell postulou que a luz, enquanto onda eletromagnética, ao ser refletida e espalhada, exerceria uma força sobre um objeto, deduzindo uma equação para tal.

Essa força foi posteriormente medida por Lebedev, em 1901, confirmando a teoria de Maxwell. Quase um século depois, mais especificamente, em 1970 ${ }^{[1]}$, Arthur Askins demonstrou que partículas dielétricas podem ser aprisionadas por apenas 2 feixes de luz e manipuladas por eles, sendo esse trabalho complementado teoricamente posterimente ${ }^{[17]}$. Depois, em $1986^{[2]}$, introduziu outro grande avanço ao conseguir com único feixe altamente focalizado, projetado por uma objetiva de grande abertura numérica, aprisionar uma partícula e movê-la em 3 dimensões. Essa técnica passou a ser conhecida como pinça óptica. A partir desse momento a pinça óptica foi então utilizada em diversos estudos, principalmente na área de Biologia depois de $1987^{[24]}$, quando Ashkin demonstrou ser possível, utilizando laser no comprimento de onda do infra-vermelho para manipular materiais como células sem danificálas. 


\subsection{Algumas aplicações da pinça óptica}

Um dos possíveis usos da pinça óptica se dá na área de microfluídica ${ }^{[19]}$, onde estudos mais recentes a utilizaram como medidor de velocidade em sistemas microfluídicos ${ }^{[20]}$, para medir peso de gotas nesse sistema ${ }^{[18]}$ ou controlar sólidos imersos em micro fluxo ${ }^{[21]}$. Outro trabalho em desenvolvimento propõe utilizar a pinça óptica no estudo de um sistema de microfluídica que simularia o tubo de extração de petróleo a altas profundidades ${ }^{[22]}$, entre outro que estudo a supençao das microesfera pelo laser para ser utilizadonos outro estudos ${ }^{[15]}$ , e o uso de chips de microfluidica para melhorar a manipulação da célula pela pinça ${ }^{[19]}$, sendo a microfluidica uma das áreas de auaçao do nosso laboratório ${ }^{[23]}$.

$\mathrm{Na}$ Biologia, a pinça óptica tem inúmeras aplicações. Ela pode ser utilizada para determinar a força do flagelo de uma bactéria ou protozoário, como, por exemplo, do Trypanosoma cruzi ${ }^{[26]}$; para inserir DNA no núcleo de uma célula ${ }^{[25]}$; ou mesmo para inserir microesferas no citoplasma de uma célula viva e movê-la. Realizando esse mesmo procedimento, pode-se mensurar a força de resistência que o citoplasma exerce, determinando sua deformabilidade. Esse processo geralmente é feito utilizando vários componentes, como nano partículas de metal, quantum dots ${ }^{[3]}$, microesferas de poliestireno e sílica ${ }^{[4]}$, ou com o uso das próprias partículas granuladas de lipídio da célula. Esta aplicação em células vivas foi realizada para estudar a difusão no citoplasma ${ }^{[6]}$, sendo feita em fibroblastos embrionários de ratos para estudar a viscosidade citoplasmática. Também foram realizados estudos de aplicação da pinça óptica para compreender as propriedades mecânicas e dinâmicas de motores moleculares ${ }^{[7]}$, além de ter sido aplicada para a caracterização mecânica e de forças em células vivas ${ }^{[8]}$.

Alem de utilizar a pinça, para estudar a dinâmica de força dos componetes internos ${ }^{[9]}$ da celula como mirotubos ${ }^{[10]}$, a sua difuçao interna ${ }^{[5]}$, e entre outras, uso delas com outras técnicas, como fluorescência ${ }^{[11]}$.

Essas são algumas das aplicações recentes, sendo possível notar que o uso da pinça contribui grandemente com o estudo das propriedades mecânicas de células.

A pesquisa aqui proposta foi realizada no laboratório de Microrreologia do Instituto de Física. Construir uma pinça óptica consiste em um processo longo e complexo, e, até o momento, não consta na literatura sua acoplagem a um sistema de Microscopia de Força e Tração (TFM). Desta forma, esse estudo buscou construir uma pinça simples e avaliar sua força teórica através do desenvolvimento de um programa para essa simulação. Durante a realização do presente estudo, realizamos os estudos teóricos, fizemos simulações computacionais, e montamos a pinça óptica e o 
sistema foi finalizado e calibrado. Este estudo permitirá futuros desenvolvimentos da técnica e associações desta com outras de medição de propriedades mecânicas de células vivas, como o TFM.

\subsection{A força da pinça óptica}

A teoria desenvolvida para explicar a força que a pinça óptica exerce sobre uma partícula é chamada de "teria geral de Lorenz-Mie", ou GLTM, em inglês. No entanto, por sua complexidade matemática, se usam outros modelos para tal. Para regimes onde o comprimento de onda é muito menor que diâmetro da partícula, utiliza-se o modelo de partícula dielétrica em um campo eletromagnético. Por outro lado, quando o diâmetro da partícula é muito maior que comprimento de onda do laser, utilizamos o modelo geométrico de força, desenvolvido por Ashkin e Ross. Para os materiais biológicos se utiliza o modelo de Lorenz-Mie. Todos esses modelos serão abordados no capítulo 2 deste trabalho, onde o estudo detalhado das teorias é realizado.

Para que se possa ter uma idéia dos valores de força envolvidos no mecanismo celular, que são da ordem de pico a femtonewtons, como, por exemplo: as forças motoras da célula cinesina e miosina, que variam de 7 a $9 \mathrm{pN}^{[4],[12],[13],[14],[16]}$; as forças envolvidas na deformação da membrana viscoelástica podem ser da ordem de dezenas de femtonewtons, onde o peso da célula menor é cerca de $10^{-15} \mathrm{Kg}^{[13],[12]}$ e uma força de 1 piconewton equivaleria a uma aceleração da ordem de $1000 \mathrm{~m} / \mathrm{s}^{2^{[12],[13][16]}}$. Desta forma, devemos considerar que trabalhar com força em femtonewtos pode ocasionar problemas, pois os ruídos ópticos costumam deturpar a força e, para valores iguais ou abaixo $20 \mathrm{fN}^{[4],[16]}$ são necessárias técnicas especiais para tal. Outro fato a ser destacado é o de que, mesmo usando o laser no comprimento do infravermelho, para forças superiores a 200 piconewtons ${ }^{[4],[16]}$, podem ocorrer danos térmicos ao material biológico. 


\subsection{Construções da pinça óptica, calibração e comparação numérica usando a plataforma Mathemática.}

No presente trabalho construímos um arranjo simplificado de pinça óptica. O propósito foi o de demonstrar a possibilidade de construção de um desenho experimental simples, controlado por um sistema eletrônico desenvolvido para esse fim. Para esta construção, utilizamos um laser de $532 \mathrm{~nm}$ de estado sólido (Solid State Laser - Coherent - $532 \mathrm{~nm}-1 \mathrm{~W}$ ) com potência ajustável e uma mesa óptica com controle pneumático de vibração (Newport). Calibramos o laser usando microesferas de poliestireno de 2,4 $\mu \mathrm{m}$ de diâmetro, com indice de refraçao de 1,59. Foi desenvolvido um programa em Mathemática para calcular a força da pinça óptica a partir de pinças já existentes ${ }^{[1]}$, usando o modelo de GLMT, que será melhor apresentado no capítulo 2, e comparamos com os valores experimentais. 


\section{Referências}

1. A. Ashkin, "Acceleration and trapping of particles by radiation pressure," Phys. Rev. Lett., vol. 24, no. 4, pp. 156-159, 1970.

2. A. Ashkin, J. M. Dziedzic, J. E. Bjorkholm, and S. Chu, "Observation of a single-beam gradient force optical trap for dielectric particles,"Opt. Lett.,vol. 11, no. 5, pp. 288$290,1986$.

3. S. Courty, C. Luccardini, Y. Bellaiche, G. Cappello, and M. Dahan, "Tracking individual kinesin motors in living cells using single quantum-dot imaging," Nano letters,vol. 6, no.7, pp. 1491-1495, 2006.

4. D. C. Appleyard, K. Y. Vandermeulen, H. Lee, and M. J. Lang, "Optical trapping for undergraduates," American Journal of Physics, vol. 75, pp. 5-14, 2007.

5. A. Caspi, R. Granek, and M. Elbaum, "Diffusion and directed motion in cellular transport," Phys. Rev. E Stat. Nonlin. Soft Matter Phys., vol. 66, pp. 011916, 2002.

6. M. Guo, A. J. Ehrlicher, S. Mahammad, H. Fabich, M. H. Jensen, J. R. Moore, J. J. Fredberg, R. D. Goldman, and D. A. Weitz, "The role of vimentin intermediate filaments in cortical and cytoplasmic mechanics," Biophysical journal,vol. 105, no.7, pp. 15621568, 2013.

7. K. Norregaard, L. Jauffred, K. Berg-Sørensenb and L. B. Oddershede, "Optical manipulation of single molecules in the living cell," Phys. Chem. Chem. Phys., vol. 16, pp. 12614-12624, 2014.

8. Y. Wu, D. Sun, and W. J. Huang, "Mechanical force characterization in manipulating live cells with optical tweezers," J. Biomech., vol. 44, no. 4, pp. 741-746, 2011.

9. B. H. Blehm, T. A. Schroer, K. M. Trybus, Y. R. Chemla and P. R. Selvin, "In vivo optical trapping indicates kinesin's stall force is reduced by dynein during intracellular transport," Proc. Natl Acad. Sci. USA,vol. 110, pp. 3381-3386, 2013.

10. A. G. Hendricks, E.L. Holzbaur, and Y.E. Goldman, "Force measurements on cargoes in living cells reveal collective dynamics of microtubule motors," Proc. Natl. Acad. Sci. USA.,vol. 109, pp. 18447-18452, 2012.

11. J. Ries and P. Schwille, "New concepts for fluorescence correlation spectroscopy on membranes," Phys. Chem. Chem. Phys., vol. 10, pp. 3487-3497, 2008.

12. A. A. R. Neves. Força óptica em pinças ópticas: estudo teórico e experimental. 2006. 169 p. Tese (doutorado) - Universidade Estadual de Campinas, Instituto de Física Gleb Wataghin, Campinas, SP.

13. B. E. A. Saleh, and M. C. Teich, Fundamentals of photonics. Wiley, 2ed., 2006.

14. Tunerblade laser optics,F.R Duarte, CRC Press, 2ed, 2017

15. R. S. Conroy, B. T. Mayers, D. V. Vezenov, D. B. Wolfe, M. G. Prentiss, and G. M. Whitesides, "Optical waveguiding in suspensions of dielectric particles,"Applied Optics, vol44, no. 36, pp. 7853-7857, 2005.

16. K. C. Neuman, and S. M. Block, "Optical trapping," Rev SciInstrum, vol. 75, no. 9, pp. 2787-2809, 2004. 
17. C. Rosen, "La levitation optique de spheres," Canadian Journal of Physics,vol. 57, pp.1260, 1979.

18. Y. Jin, A. Orth, E. Schonbrun, and K. B. Crozier, "Measuring the pressures across microfluidic droplets with an optical tweezer,"Optics Express, vol. 20, no. 22, pp. 24450, 2012.

19. X. Wang, S. Chen, M. Kong, Z. Wang, K. D. Costa, R. A. Li, and D. Sun, "Enhanced cell sorting and manipulation with combined optical tweezer and microfluidic chip technologies,'Lab on a Chip, vol. 11, no. 21, pp. 3656-3662, 2011.

20. C. L. Lin, I. Wang, B. Dollet, and P. L. Baldeck, "Velocimetry microsensors driven by linearly polarized optical tweezers,”Optics Letters, vol. 31, no. 3, pp. 329-331, 2006.

21. A. L. Birkbeck, S. Zlatanovic, S. C.Esener, and M.Ozkan, "Laser-tweezer-controlled solid immersion microscopy in microfluidic systems,"Optics Letters, vol. 30, no. 20, pp. 2712-2714, 2005.

22. A. T. da Cunha Lima, I. C. da Cunha Lima, and M. P. de Almeida,"Analysis of turbulence power spectra and velocity correlations in a pipeline with obstructions,'International Journal of Modern Physics C, vol. 28, no. 2, pp. 1750019, 2017.

23. J. E. R. Cervantes. Estudo experimental e computacional sobre a geração de macroemulsões e outros sistemas mediante dispositivos microfluídicos. 2017. 114 p. Dissertação (mestrado) - Universidade de São Paulo, Instituto de Física, São Paulo, SP.

24. A. Ashkin, J. M.Dziedzic, and T. Yamane, "Optical Trapping and Manipulation of Single Cells Using Infrared-Laser Beams,’Nature, vol. 330, no. 6150, pp. 769-771, 1987.

25. M. Waleed, S. Hwang, J. Kim, I. Shabbir, S. Shin, and Y. Lee, "Single-cell optoporation and transfection using femtosecond laser and optical tweezers," Biomed. Opt. Express, vol. 4, pp. 1533-1547, 2013.

26. E. Stellamanns, S. Uppaluri, A. Hochstetter, N. Heddergott, M. Engstler, and T. Pfohl, "Optical trapping reveals propulsion forces, power generation and motility efficiency of the unicellular parasites Trypanosomabruceibrucei,"Scientific Reports, vol. 4, pp. 1-7, 2014. 


\section{Capítulo 2}

\section{Teoria}

\subsection{Introdução à teoria da força da pinça óptica}

Desde o primeiro aprisionamento de uma microesfera através de um feixe de luz, realizado em 1986 por Ashkin e colaboradores, a pinça óptica tem apresentado grande utilidade em diversas áreas, principalmente na Biologia e no estudo de nano partículas. A primeira idéia de que a luz possa prender uma partícula está no conceito de que a luz pode ser representada como uma partícula, um fóton, que possui um momento específico calculado por:

$$
p=-\hbar c ̧ \frac{d}{d x}=\frac{h}{\lambda}=n \frac{E_{f}}{c}
$$

onde $\lambda$ representa o comprimento da onda, ç representa uma constante de normalização, e $E_{f}$ a energia do fóton com frequência $f$. A luz que colide com uma esfera transfere momento a ela, e, ao atingir o centro espalhador, recebe impulso na direção da bissetriz do ângulo entre o fóton incidente e o refratado, como se ocorresse um choque entre partículas, como esquematizado na Figura 2.1.

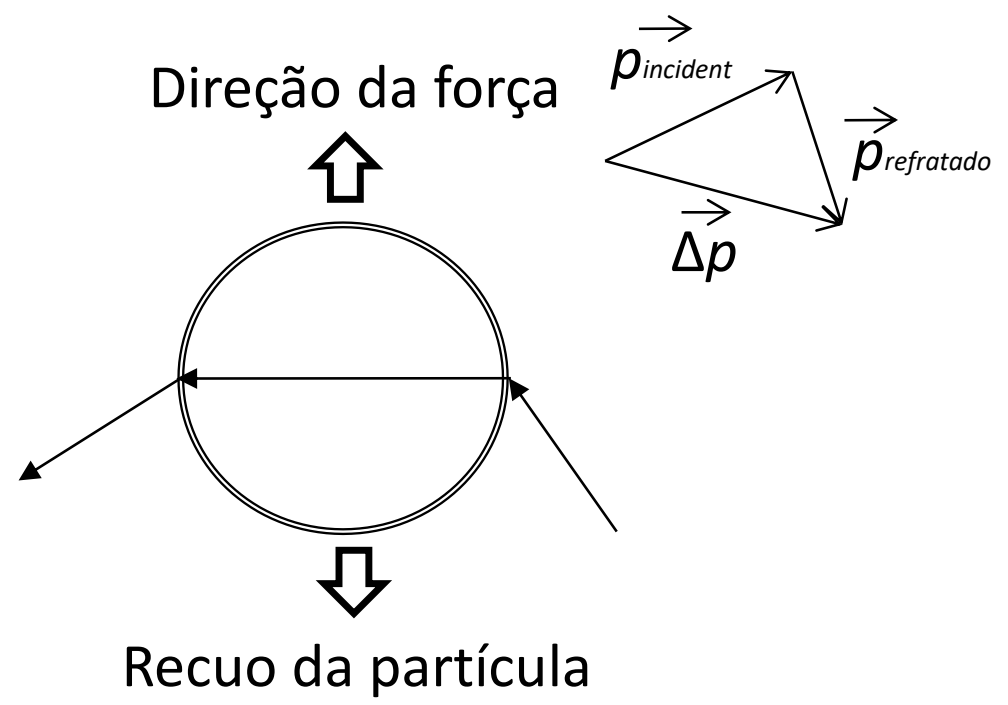

Figura 2.1: Representação do momento do feixe que, ao ser refratado pela partícula, exerce uma força e sofre recuo ao transferir momento para ela.

De acordo com a segunda lei de Newton, a variação de momento resulta numa força na direção desse vetor, ou seja, podemos escrever que o módulo da força para uma reflexão total da luz é: 


$$
F=\frac{\Delta p}{\Delta t}=2 n_{2} \frac{Q P}{c}
$$

onde $\mathrm{P}$ representa a potência do laser, $n_{2}$ representa o índice de refração do meio, e Q representa o fator de qualidade de transferência da potência. Para $n_{2}=1$, o índice do ar, temos força da ordem de $10 \mathrm{nN}$, no entanto, por conta do fator de qualidade $\mathrm{Q}$ ser, na maioria das vezes, 0,2, a força da armadilha é da ordem de 0,01 a $100 \mathrm{pN}$, onde Q depende da abertura numérica da objetiva, do índice de refração relativo, do comprimento, polarização e da geometria da partícula. Logo, considerando a pinça óptica sendo composta por 2 raios que atravessam uma esfera como apresentado nas Figuras 2.2 e 2.3 , onde $n_{1}$, que representa o índice de refração da esfera, e $n_{2}$, o do meio, e sendo esse raios simétricos, quando $n_{1}>n_{2}$ tende a trazer a esfera para o foco do laser, capturando-a, e, quando $n_{1}<n_{2}$, tende a

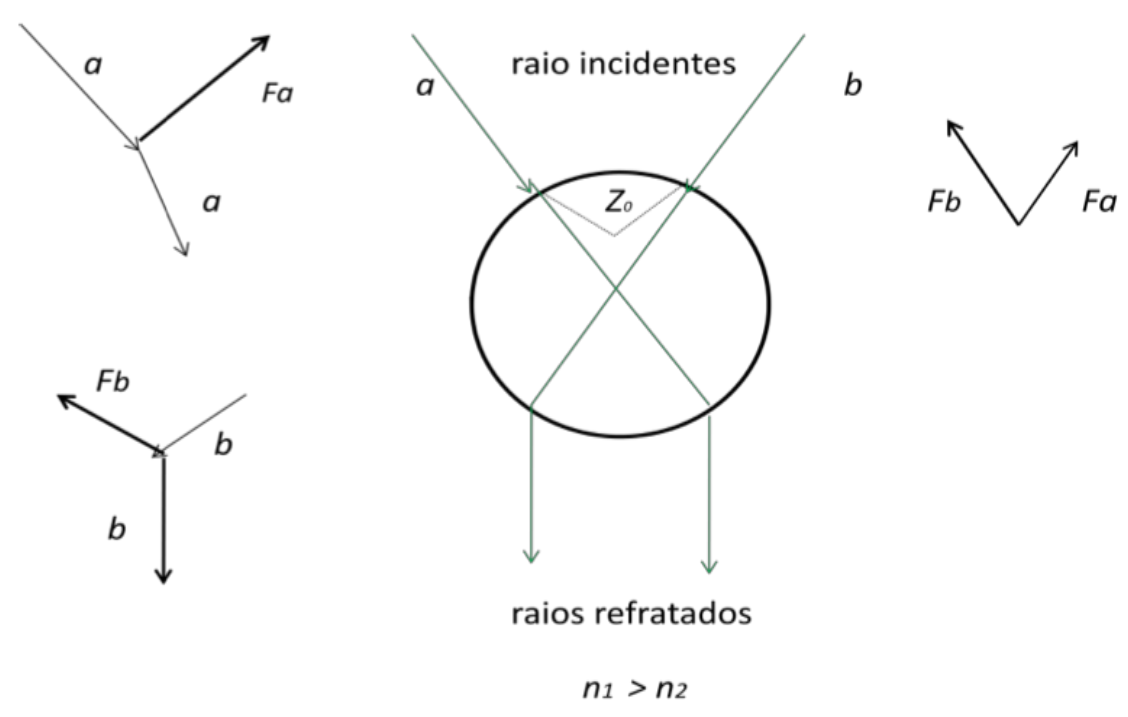

expulsar a esfera para fora do foco.

Figura 2.2: Representação dos raios refratados ao passar por uma esfera dielétrica com índice de refração da esfera maior que o índice de refração do meio. 

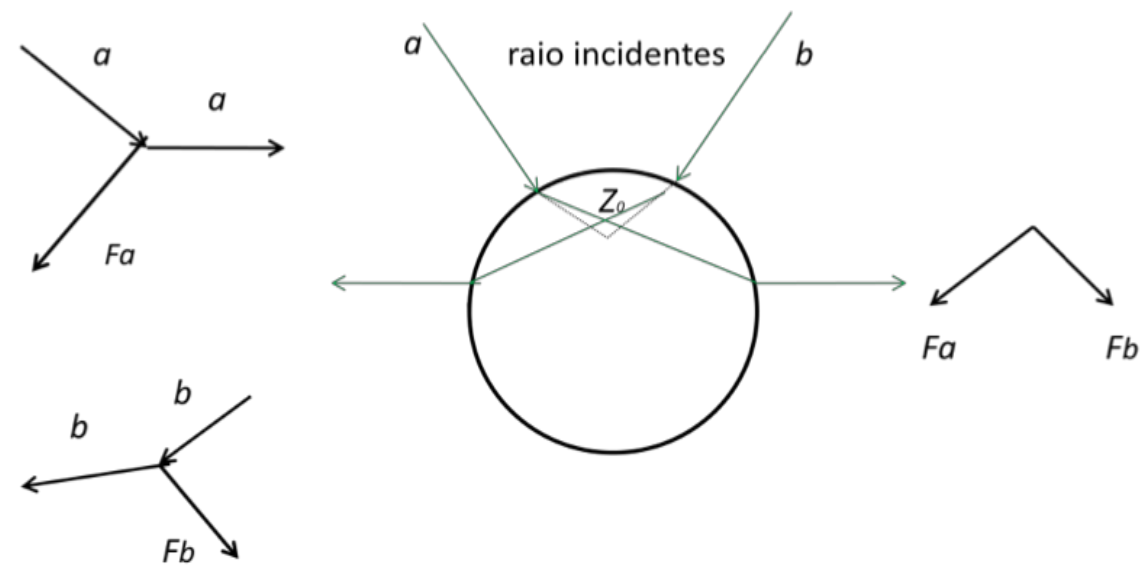

raios refratados

$$
n_{1}<n_{2}
$$

Figura 2.3: Representação dos dois feixes de laser sendo refratados pela esfera com índice de refração do meio maior que o índice de refração da esfera.

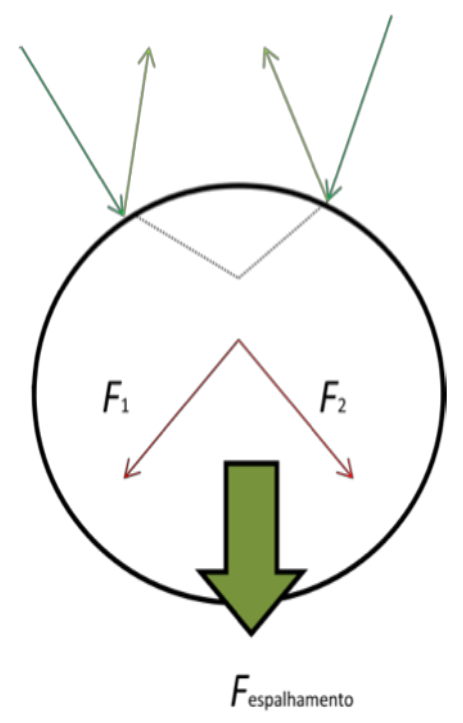

Figura 2.4: Representação da força de espalhamento que é representada pela somatória da força de recuo $F_{1}$ e $F_{2}$ somada à força resultante da absorção de parte dos raios refletidos, representados pela cor verde claro na imagem. 
Outro fato a salientar é o de que, além da força exercida sobre a esfera pela refração, que chamamos de força de gradiente, a força exercida sobre reflexão e pela absorção da luz do laser, chamada de força de espalhamento, tende a empurrar a esfera do foco, logo, a pinça óptica só pode existir quando a força do gradiente é maior que força de espalhamento.

Para calcular a real força exercida existem três teorias que relacionam as dimensões da partícula com o comprimento de onda da luz. Essas teorias são: Rayleigh, para partículas de diâmetro $d<<\lambda$; teoria de Lorenz-Mie, para partículas com $d \cong \lambda$; e a teoria de raios ópticos, para $d>>\lambda$. A teoria de Lorenz - Mie é considerada a mais geral, podendo ser usada para descrever a força nas três situações, porém, dada sua complexidade, optamos por explicar as forças nos outros dois regimes, utilizando sua teria de força original para facilitar o entendimento. A teoria descrita nessa seção se aplica ao regime óptico, sendo que, nos dois outros regimes temos outra teoria para a força de espalhamento e gradiente, que será apresentada nas próximas seções.

\subsection{Teoria da força em regime $(d \ll \lambda)$ ou Rayleigh}

O regime de Rayleigh é considerado quando o diâmetro da partícula aprisionada é muito menor que o comprimento de onda do laser. Portanto, para se considerar esse regime, a seguinte relação matemática deve ser válida $2 k d\left(\mu_{n}-1\right) \ll 1$, onde $k=\frac{2 \pi}{\lambda}$ representa o número de onda no meio, $d$ o raio da partícula, e $\mu_{n}=n_{1} / n_{2}$, onde $n_{2}$ e $n_{1}$ representam os índices de refração do meio e da partícula, respectivamente. Além disso, vamos considerar aqui a partícula como uma esfera. Mesmo outras partículas e moléculas, com formato não esférico, quando consideradas como uma esfera podem apresentar boa aproximação. Considerando também que material é dielétrico, como a maioria das microesferas que utilizamos, esse torna-se um sistema clássico de uma partícula dielétrica imersa num campo eletromagnético. É necessário ainda considerar a força de espalhamento de Mie descrita na literatura ${ }^{[3][5][6][12]}$, onde a luz atua como um "fluído", aplicando um momento na partícula; essa derivação de Mie é diferente da que será apresentada na Secção 2.4, sobre o cálculo da força de Mie, contudo, não a descreveremos aqui. Todavia, o leitor pode segui-la pelas referências ${ }^{[6][12]}$. Essas duas forças são chamadas de força de gradiente e força de espalhamento, onde a força de gradiente fixa a esfera no plano focal, enquanto a de espalhamento, com sentido e direção do vetor $\vec{k}$, tende a retirá-la. Para situações ideais, a força de espalhamento tem que ser bem menor do que a de gradiente para adequada fixação.

A força do gradiente, já deduzida ${ }^{[4]}$, representa a força que um campo eletromagnético aplica numa partícula dielétrica e é dada pela força de Lorenz: 


$$
\vec{F}_{G}=(\vec{p} \cdot \nabla) \vec{E}+\frac{\partial \vec{p}}{\partial t} \times \vec{B}
$$

onde $\vec{p}=\vec{d} q$ é o vetor do momento do dipolo, que também pode ser escrito como $\vec{p}=\vec{E} \tau$, sendo $\vec{E}$ e $\vec{B}$ campos elétrico e magnético, respectivamente.

Utilizando a identidade vetorial $(\vec{E} \cdot \nabla) \vec{E}=\nabla\left(\frac{E^{2}}{2}\right)-\vec{E} \times \nabla \times \vec{E}$, e a equação de Maxwell

$$
\vec{E} \times \nabla+\frac{\partial \vec{B}}{\partial t}=0
$$

Podemos, então, reescrever a Equação (2.3) como

$$
\vec{F}_{G}=\tau\left[\nabla\left(\frac{E^{2}}{2}\right)+\frac{\partial}{\partial t}(\vec{E} \times \vec{B})\right]
$$

Supondo que partícula não tenha uma movimentação, ou que seja muito pequena, num feixe contínuo de laser não há variação no tempo, logo, a derivada temporal se anula e, então, a força óptica é proporcional ao gradiente de intensidade do campo elétrico. Desta forma, a Equação (2.5) fica

$$
\left\langle\vec{F}_{G}\right\rangle=\tau \nabla\left\langle E^{2}\right\rangle / 2
$$

Considerando que a esfera dielétrica não possua nenhuma carga livre, nem dentro ou fora, só há a ação do campo (Figura 2.5), então, acatamos que o campo é uma onda que satisfaz a equação de Laplace, que trataremos nas Seções 2.4.1 e 2.4.2, onde as equações de ondas são de simetria axial, o que nos fornece que o valor da constante de dipolo elétrico, $\tau \mathrm{e}^{[3],[4]}$,

$$
\tau=4 \pi n_{2} d^{3}\left(n_{1}^{2}-n_{2}^{2}\right) /\left(n_{1}^{2}+2 n_{2}^{2}\right)
$$

onde $d$ representa o raio da partícula.

Nessa equação, se $n_{1}>n_{2}$ a força é positiva ou atrativa, se $n_{1}<n_{2}$ a força será negativa ou repulsiva, além de ser proporcional ao volume da partícula. Contudo, para determinar a força de espalhamento, consideramos que a densidade do pseudomomento ${ }^{[8]}$ eletromagnético do raio de luz é:

$$
G=\langle\vec{S}\rangle_{t} / c^{2}
$$

onde $\langle\vec{S}\rangle$ é o vetor de Pointing médio no tempo, logo, a força de espalhamento pode ser representada por ${ }^{[3],[5],[8]}$ :

$$
\vec{F}_{e s p}=\left(\frac{\langle\vec{S}\rangle_{t}}{c}\right) n_{2} C_{p r}
$$


onde $C_{p r}$ representa o coeficiente da secção de choque da pressão da onda eletromagnética ao colidir na esfera e se espalhar, que foi determinada por Mie, e, após sua dedução, obtêm-se a seguinte expressão ${ }^{[3][5][6][12]}$,

$$
C_{p r}=\frac{8}{3} \pi k^{4}\left(n_{1}^{2}-n_{2}^{2}\right) /\left(n_{1}^{2}+2 n_{2}^{2}\right) d^{6}
$$

sendo, então a Equação 2.9 análoga a Equação 2.2, onde temos que $\langle\vec{S}\rangle$ seria a potência $P$, e $C_{p r}$ o fator de qualidade Q. Aqui, a força seria provocada por uma onda colidindo com o campo elétrico análogo, como onda sonora colidindo com objeto material. $E^{2}$ será uma função multipolo igual às descritas mais à frente nas Equações 2.118 e 2.119, e, sendo que, os coeficientes de expansão de espalhamento serão muito maiores em valores do que os coeficientes de forma de onda, resultando em uma equação diferente de força, equação essa, próxima às Equações 2.131 e 2.132, às quais não serão apresentadas em detalhes, pois não foi realizado nenhum experimento utilizando a força nesse regime.

Logo, para pinça prender uma partícula, a condição necessária é $\left\langle\vec{F}_{G, Z}\right\rangle-\vec{F}_{e s p}>0$, condição essa, apresentada no funcionamento da pinça óptica.

Desta maneira, as dificuldades experimentais para obtenção desse regime se dão na má focalização de um raio tão pequeno, e no alinhamento que deve ser bem sensível, dadas as distâncias nano métricas. Tais condições dificultam muito a construção de uma armadilha para partículas menores que $100 \mathrm{~nm}{ }^{[2]}$. Além disso, a partícula presa por laser depende de sua polarizabilidade, onde, para se prender uma partícula de $10 \mathrm{~nm}$ de raio é necessário um potencial inicial muito maior do que para prender uma partícula de $1 \mu m^{[2]}$. Para partículas de $200 \mathrm{~nm}$, a força regime de Mie exerce uma força não desprezível, causando diferenças consideráveis nos valores da força obtidas experimentalmente para laser com comprimentos de ondas visíveis, ou seja, para valores acima desse valor o tratamento de Rayleigh não mais se aplica.

Pode-se também aplicar outras técnicas para aprimorar as armadilhas, como reduzir a temperatura do meio, além de aplicar alto vácuo, para tentar minimizar os efeitos de outras forças na partícula e, assim, obter uma melhor medida da força no regime de Rayleigh. Para partículas menores que $5 \mathrm{~nm}$, são utilizados aprimoramentos ${ }^{[2]}$, como ressonância de plasma e iônica, ou intensidade dependente do índice de refração, para aumentar à constante dielétrica, fazendo com que laser com potências muito grandes não sejam necessários. Essas medidas são difíceis de aplicar, e, portanto, a maioria das medidas experimentais da força nesse regime acaba desviando do esperado. 


\subsection{Teorias para regime óptico $(\mathrm{d} \gg \lambda)$}

Ashkin e Rose foram os primeiros a determinar a força que age sobre uma partícula no regime óptico, onde, nesse regime, cada raio se propaga de forma individual, com sua potência, intensidade, direção e estado de polarização, sendo sua propagação uniforme se o material tiver índice de refração homogêneo ao longo de seu comprimento. Além disso, cada raio possui características de uma onda de comprimento zero, podendo mudar sua direção toda vez que refletida e refratada, podendo, também, mudar sua polarização de acordo com a equação de Fresnel.

Desta forma, usamos esse modelo em que a força da armadilha óptica será igual à força de cada raio individual. Nesta situação, a direção da força é calculada através da trajetória dos raios, sendo que os raios de estrutura e polarização indefinida entram na objetiva de grande abertura numérica, e focalizam um ponto unidimensional, como esquematizado na Figura 2.6, sem aberrações esféricas. Vamos considerar que o raio atinge a bead com ângulo $\alpha$, como apresentado nas Figuras 2.5 e 2.6, estando este se propagando ao longo do eixo " $y, z$ ", e considerando zero as contribuições para o eixo $x$. Negligenciamos também o fato de que o ponto focal não é um ponto unidimensional, mas sim com dimensão finita em 3 dimensões, ou seja, usamos que para o limite ${ }^{[8]}$ de $\lambda / 2 n_{2}$, muito menor que raio $d$ da esfera, o ponto focal da esfera é unidimensional idealizado. Além disso, no ponto focal em que os raios convergem, as direções e momento dos raios continuam em linha reta, sendo que a força se dá pela interação com a matéria que é a reflexão e a refração do feixe de luz.

Ao considerar um raio com potência $P$ que atinge uma esfera dielétrica, como na Figura 2.5, podemos deduzir os componentes da força que raio aplica na esfera ${ }^{[10]}$, onde, o raio inicialmente age com uma força igual à descrita na Equação (2.2) e depois é refratado e refletido infinitas vezes. Dependo do ângulo $\alpha$ de incidência, podemos calcular para as duas componentes $y$, nas duas forças:

$$
\begin{aligned}
& F_{z}=\frac{n_{2} P}{c}-\left[\frac{n_{2} P R}{c} \cos (\pi+2 \alpha)+\sum_{n=0}^{\infty} \frac{n_{2} P T^{2} R^{n}}{c} \cos \left(\alpha^{\prime}+n \beta^{\prime}\right)\right] \\
& F_{y}=-\left[\frac{n_{2} P R}{c} \operatorname{sen}(\pi+2 \alpha)+\sum_{n=0}^{\infty} \frac{n_{2} P T^{2} R^{n}}{c} \operatorname{sen}(\alpha+n \beta)\right]
\end{aligned}
$$

Agora, considerando que a força no eixo $x$ é zero, podemos somar as força num plano complexo $F_{t o t}=F_{z}+i F_{y}$, desta forma, somando as equações:

$$
F_{\text {tot }}=\frac{n_{2} P}{c}[1+R \cos 2 \alpha]+i \frac{n_{2} P}{c} R \operatorname{sen} \alpha \frac{n_{2} P}{c} T^{2} \sum_{n=0}^{\infty} R^{n} e^{i\left(\alpha^{\prime}+n \beta^{\prime}\right)}
$$

A soma de série geométrica simples tem o fator de 


$$
F_{\text {tot }}=\frac{n_{2} P}{c}[1+R \cos 2 \alpha]+i \frac{n_{2} P}{c} R \operatorname{sen} \alpha-\frac{n_{2} P}{c} T^{2} e^{i \alpha^{\prime}}\left[\frac{1}{1-R e^{i \beta^{\prime}}}\right]
$$

onde os valores de $\alpha^{\prime}=2 \alpha-2 \beta$, e $\beta^{\prime}=\pi-2 \beta$, chegando à expressão:

$$
F_{\text {tot }}=\frac{n_{2} P}{c}\left[1+e^{i 2 \alpha}-T^{2}\left[\frac{e^{i 2(\alpha-\beta)}}{1+R e^{i 2 \beta}}\right]\right]=\frac{n_{2} P}{c} Q_{T}
$$

Nessa expressão, $T$ e $R$ são os coeficientes de transmissão e reflexão na esfera, que mudam de acordo com material que a constitui, e os coeficientes dependem dos ângulos $\alpha, \beta$ e do tipo de incidência no raio da esfera: se são transversal magnético (TM) ou transversal elétrico (TE). Assim, calculando a média para os dois casos, temos:

$$
\begin{array}{r}
R(\alpha, \beta)=\frac{1}{2}\left[\frac{\operatorname{sen}(\alpha-\beta)}{\operatorname{sen}(\alpha+\beta)}\right]^{2}+\frac{1}{2}\left[\frac{\tan (\alpha-\beta)}{\tan (\alpha+\beta)}\right]^{2} \\
\mathrm{~T}(\alpha, \beta)=1-R(\alpha, \beta)
\end{array}
$$

Logo, ainda nos falta determinar a direção e o sentido da força em relação as coordenadas cartesianas, que serão abordados na próxima seção.

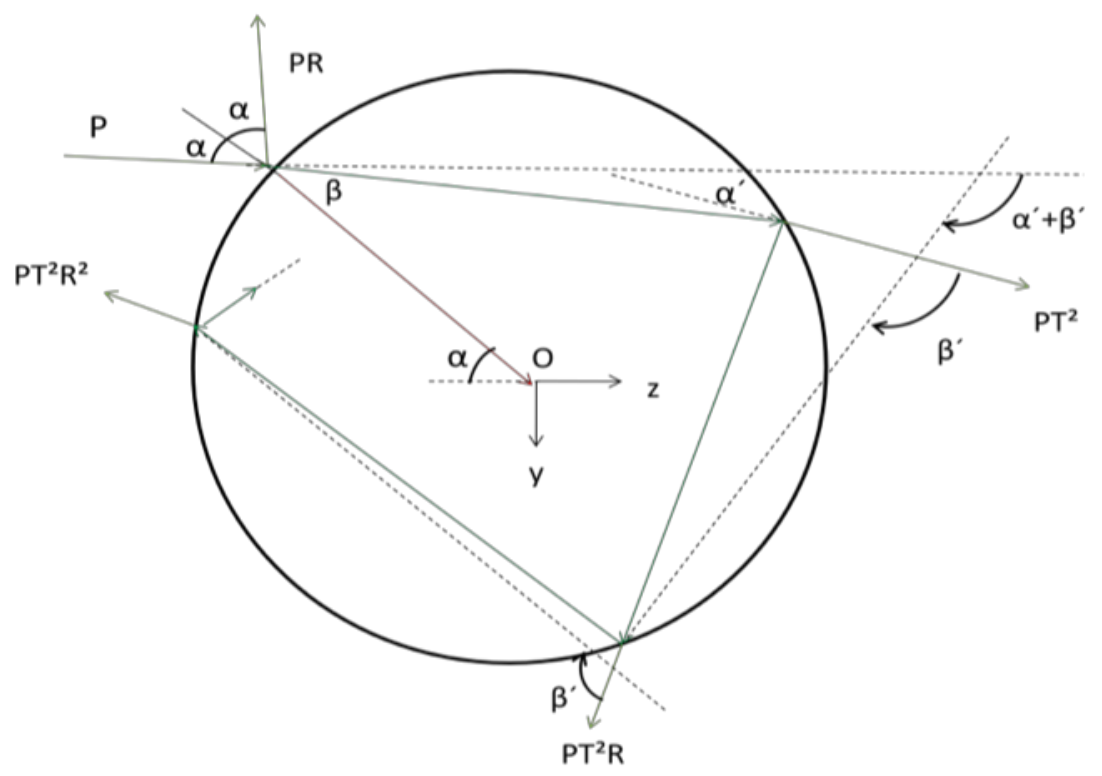

Figura 2.5: Percurso do raio de luz ao entrar na esfera onde a força é dada pela potência coeficiente de reflexão e transmissão. 
2.3.1 Determinar os ângulos e versores da força sem considerar as aberrações esféricas.

Uma vez que foi possível determinar as direções em que o feixe entra na esfera e seus ângulos, sendo esse processo já demonstrado em outros trabalhos ${ }^{[9],[17],[18]}$, e, considerando a esfera anterior e sua relação ao novo eixo cartesiano $\mathrm{z}, \mathrm{x}^{\prime}$, onde $\mathrm{o}$ raio atinge a esfera no ângulo $\alpha$ e na direção dos eixos anteriores, temos, então, a figura abaixo.

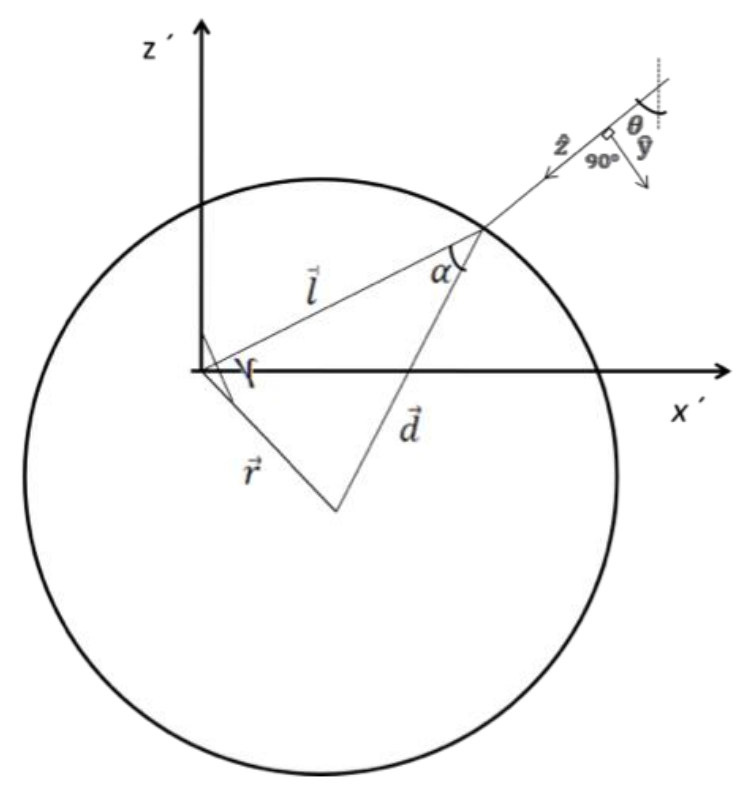

Figura 2.6: Representação de feixe de laser focado no interior da esfera sem aberrações esféricas.

Primeiramente, para determinar a força é preciso considerar que todos os raios que saem da objetiva atingem a esfera, o que não acontece, pois existe o fenômeno de aberração esférica. Neste, os raios de luz atingem a esfera em uma região do eixo z, e não num simples ponto, onde cada um destes raios possuem um ângulo de incidência diferente.

Logo, deduzimos primeiramente a força numa esfera sem aberração e, posteriormente, na Secção 2.3.2, a deduziremos para o sistema com aberração. Ao considerar uma esfera como a apresentada na Figura 2.7, temos que o raio se concentra num ponto após percorrer uma distância $\vec{l}$, 
na microesfera, sendo $\vec{d}$ o raio da esfera e $\vec{r}$ a distância em que os raios convergem no centro da microesfera. Considerando que não haja deslocamento em $x^{\prime}$, ou seja, não há nenhuma força externa atuando sobre a microesfera como, por exemplo, fluxo de água, a partícula estaria somente movendo-se em relação ao eixo z', em decorrência da força óptica. Assim, considerando os vetores escritos em coordenadas esféricas temos:

$$
\vec{l}=l(\operatorname{sen} \theta \cos \phi+\operatorname{sen} \theta \sin \phi+l \cos \theta)
$$

Escolhemos um plano $y^{\prime}=0$ para $\vec{r}$

$$
\vec{r}=r(\operatorname{sen} \gamma, 0, \cos \gamma)
$$

Sendo então $\vec{d}=|\vec{l}-\vec{r}|$ então temos que

$$
l^{2}+r^{2}-2 l r(\operatorname{sen} \gamma \operatorname{sen} \theta \cos \phi+\cos \gamma \cos \theta)=d^{2}
$$

Resolvendo essa equação de segundo grau chegamos em,

$$
l=\left[d^{2}-r^{2}+r^{2}(\operatorname{sen} \gamma \operatorname{sen} \theta \cos \phi+\cos \gamma \cos \theta)\right]^{\frac{1}{2}}+r(\operatorname{sen} \gamma \operatorname{sen} \theta \cos \phi+\cos \gamma \cos \theta)
$$

Da Figura 2.6 podemos reescrever o ângulo

$$
r^{2}=l^{2}+d^{2}-2 d l \cos \alpha
$$

onde,

$$
\alpha=\operatorname{arcos}\left[\frac{l^{2}+d^{2}-r^{2}}{2 l d}\right]^{-1}
$$

Então, usamos a lei de Snell-Decartes para calcular o ângulo $\beta$.

$$
n_{2} \sin \alpha=n_{1} \sin \beta
$$

Onde $n_{1}$ e $n_{2}$ são os mesmos da secção anterior, ou seja, o índice da esfera e do meio. Nessa equação expressamos $\alpha$ e $\beta$ em função de $\theta, \phi$, e, dessa forma, o módulo da força de único raio sobre a microesfera fica em função de paramentos únicos. Para determinar a direção e o sentido da força precisamos detrminar seus componentes em cada direção, para isso, consideramos a força como zero no eixo $\mathrm{x}$ e fixamos que a força atua apenas em " $z, y$. ."

$$
F=F_{z}+i F_{y}
$$

Logo,

$$
\vec{F}=\frac{n_{2} P}{c}\left[\operatorname{Re}\left(Q_{t}\right) \hat{z}+\operatorname{Im}\left(Q_{t}\right) \hat{y}\right]
$$

onde $P$ representa a potência do laser, e c a velocidade da luz. Por fim, temos que escrever os vetores unitários $\hat{z}, \hat{y}$ em função dos parâmetros conhecidos. Note que $\hat{z}$ está na mesma direção de $\vec{l}$, mas com sentido oposto, 


$$
\hat{z}=(-\operatorname{sen} \theta \cos \phi-\operatorname{sen} \theta \operatorname{sen} \phi-\cos \theta)
$$

O vetor $\vec{r}$ está no ponto $y^{\prime}=0$

$$
\hat{y}=\frac{\hat{z} \times(\vec{r} \times \hat{z})}{|\hat{z} \times(\vec{r} \times \hat{z})|}
$$

Podemos considerar a força de cada raio como a diferencial em relação aos ângulos $\theta, \phi, \log 0$, para sabemos a força do laser na esfera, integramos a Equação 2.26 em relação à esses ângulos $\phi, \theta$ que variam entre $0<\phi<2 \pi$ e $0<\theta<\theta_{m}$, onde $\theta_{m}=62,46^{[9],] 18]}$ é o ângulo limite da interfase água/vidro e, para valores acima, os raios são refletidos.

\subsection{2 Ângulos e versores para o caso com aberração esférica}

(a) Sem aberração

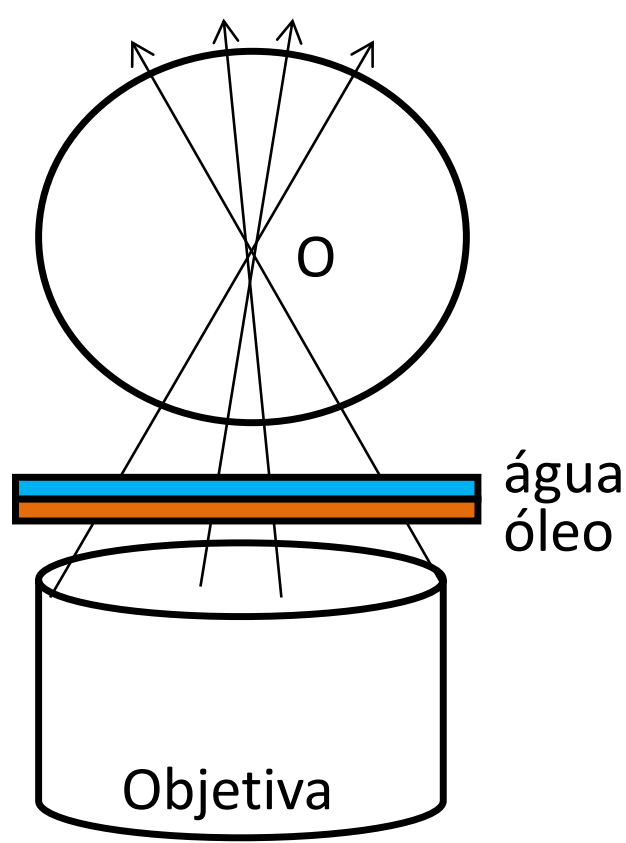

(b) Com aberração

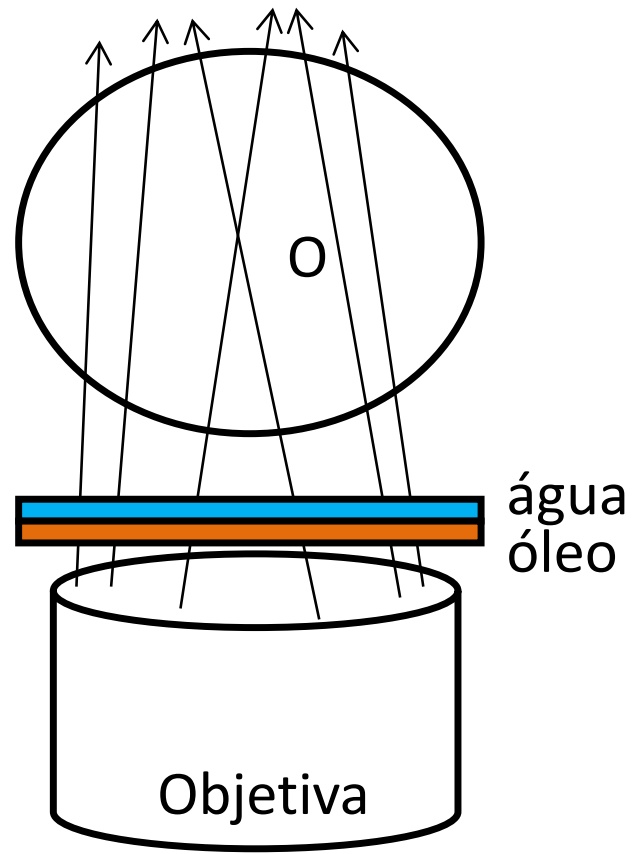

Figura 2.7: Comparação entre o foco do laser ao passar por meio de óleo e água nos casos (a) sem aberração, e (b) com aberração esférica.

Agora, consideremos como aberrações esféricas quando nem todos os raios atingem a esfera e, além disso, dos que a atingem, nem todos a atingem em seu foco, sendo, então, uma aberração focal. Outro problema apresentado nesta situação é $\theta_{m}$, o ângulo limite da interface água/vidro, que não é o mesmo da situação sem aberração. Contudo, precisamos desse ângulo 
pois, integramos o raio em relação a ele, logo, para determiná-lo, é utilizado o processo descrito no Apêndice B.

Quanto ao problema da distância focal, podemos contorná-lo pensado num sistema como o apresentado na Figura 2.8. Com esse sistema, podemos escrever a força de cada raio para o respectivo sistema, como fizemos no caso anterior. Nesse sistema começamos a definir dois eixos, que serão os mesmo da Figura $2.6, \hat{z}, \hat{y}$ que definem as direções do raio incidente, posteriormente, definimos novos eixo particulares $\hat{z}^{\prime \prime}, \hat{y}^{\prime \prime}$ que representam a direção do feixe refratado pela água ou óleo e o eixo perpendicular a ele. O vetor $\vec{r}^{\prime \prime}$ que representa o vetor que une a origem do novo centro de coordenadas da partícula ao centro da esfera, $\vec{l}^{\prime \prime}$ é a distância entre o ponto onde raio atinge a esfera e o novo centro do sistema de coordenadas da partícula, $\vec{d}^{\prime \prime}$ que representa o raio da esfera onde $\left|\vec{d}^{\prime \prime}\right|=d$, o novo ângulo $\gamma^{\prime \prime}$ que é o ângulo entre o vetor $\vec{r}^{\prime \prime}$ em relação ao eixo z', $\vec{b}$ que é o vetor da distância de um centro de coordenadas ao outro, e, por fim, $h$ que representa a distância do centro da esfera ao ponto que o raio deixa a objetiva.

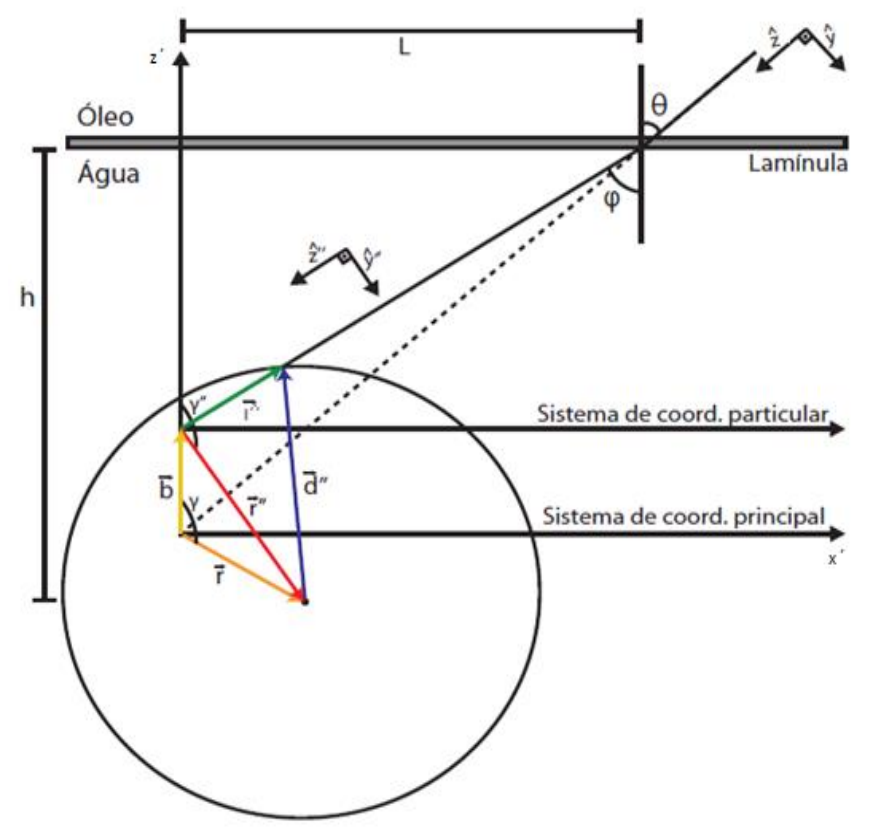

Figura 2.8: Representações de esfera atingida por feixes em outros focos, sendo o foco deslocado. A partir dos novos vetores podemos inferir a direção da nova força devida aberração esférica. Figura adaptada a partir de 
Sendo assim, a partir da Figura 2.9, acima, podemos extrair as equações.

$$
\begin{aligned}
& \tan \varphi=\frac{L}{h-b-r \cos (\pi-\gamma)} \\
& \tan \theta=\frac{L}{h-r \cos (\pi-\gamma)}
\end{aligned}
$$

Dividido a Equação 2.29 pela Equação 2.30, temos:

$$
\frac{\operatorname{sen} \theta \cos \varphi}{\cos \theta \operatorname{sen} \varphi}=\frac{1}{1+\frac{b}{h-b+r \cos \gamma}}
$$

Usando lei de Snell-Decartes, temos que:

$$
\frac{n_{2} \cos \varphi}{n_{3} \cos \theta}=\frac{1}{1+\frac{b}{h-b r \cos \gamma}}=1-\frac{b}{h+r \cos \gamma}
$$

onde $n_{3}$ é o índice de refração do vidro e $n_{2}$ o índice de refração da água. A partir da Equação 2.32 podemos escrever equação do modulo do vetor $\vec{b}$,

$$
\vec{b}=\left(1-\frac{n_{2} \cos \varphi}{n_{3} \cos \theta}\right)(h+r \cos \gamma) \hat{z}
$$

Usando a lei de Snell-Descartes podemos eliminar a dependência em $\varphi$.

$$
\vec{b}=\left(1-\sqrt{\frac{\left(\frac{n_{2}}{n_{1}}\right)^{2}-\sin ^{2} \theta}{1-\sin ^{2} \theta}}\right)(h+r \cos \gamma) \hat{z}
$$

No sistema de coordenadas particulares, analogamente ao caso sem aberração, podemos escrever

$$
\begin{gathered}
l^{\prime \prime}=\left[d^{2}-r^{\prime \prime 2}+r^{\prime \prime 2}\left(\operatorname{sen} \gamma^{\prime \prime} \operatorname{sen} \theta \cos \phi+\cos \gamma^{\prime \prime} \cos \theta\right)\right]^{\frac{1}{2}}+ \\
\mathrm{r}^{\prime \prime}\left(\operatorname{sen} \gamma^{\prime \prime} \operatorname{sen} \theta \cos \phi+\cos \gamma^{\prime \prime} \cos \theta\right) \\
\alpha=\cos \left[\frac{l^{\prime \prime 2}+d^{2}-r^{\prime \prime 2}}{2 l^{\prime \prime} d}\right]^{-1}
\end{gathered}
$$

Agora podemos escrever $\vec{r}^{\prime \prime}$ e $\gamma^{\prime \prime}$ em função de $\vec{r}$ e $\gamma$

$$
\vec{r}^{\prime \prime}=\vec{r}-\vec{b}
$$

Em módulo,

$$
r^{\prime \prime}=\sqrt{r^{2}+b^{2}-2 r b \cos \gamma} .
$$

Fazendo o produto escalar de $\vec{r}^{\prime \prime} \operatorname{com} \vec{b}$ da equação temos:

$$
\vec{r}^{\prime \prime} \cdot \vec{b}=\vec{r} \cdot \vec{b}-\vec{b} \cdot \vec{b}
$$

e desta equação e a partir da 2.37 determinamos $\gamma^{\prime \prime}$

$$
\cos \gamma^{\prime \prime}=\frac{r \cos \gamma-b}{\vec{r}^{\prime \prime}}
$$

Então, análogo ao caso sem aberração, basta escrever os vetores unitários $\hat{z}^{\prime \prime}$ e $\hat{y}^{\prime \prime}$ : 


$$
\begin{aligned}
& \hat{z}^{\prime \prime}=(-\operatorname{sen} \varphi \cos \phi,-\operatorname{sen} \varphi \cos \phi,-\cos \varphi) \\
& \text {, e } \\
& \hat{y}^{\prime \prime}=\frac{\hat{z^{\prime \prime} \times\left(\vec{r}^{\prime \prime} \times \hat{z}^{\prime \prime}\right)}}{\left|\hat{z^{\prime \prime} \times\left(\vec{r}^{\prime \prime} \times z^{\prime \prime}\right)}\right|}
\end{aligned}
$$

A partir desse resultado podemos calcular a força total sobre a microesfera considerando a Equação 2.36. Considerando a força devido a um raio, a equação diferencial da força total do feixe é dada por:

$$
d \vec{F}=\frac{n_{2}}{c}\left[\operatorname{Re}\left(Q_{z}\right) \hat{z}^{\prime \prime}+\operatorname{Im}\left(Q_{z}\right) \hat{y}^{\prime \prime}\right] d P
$$

Agora, é necessário escrever $d P$ em função de parâmetros conhecidos, como a intensidade do feixe $I A=P$, onde $A$ é a área do laser que fornece a potência. A intensidade do feixe varia de padrão, mas, como a maior parte dos lasers são gaussianos, então:

$$
I(\rho)=I_{0} \exp \left(\frac{-\rho^{2}}{2 \sigma^{2}}\right)
$$

Sendo $\sigma$ a largura da entrada da objetiva.

Sabendo que $d P=I d A$ e para a área consideramos coordenadas cilíndricas, $d A=\rho d \rho d \phi$, também usando a Equação 2.44, podemos escrever:

$$
d P=I_{0} \exp \left(\frac{-\rho^{2}}{2 \sigma^{2}}\right) \rho d \phi d \rho
$$

Integrando a equação em relação a $0<\phi<2 \pi$, $e 0<\rho<\infty$

$$
P=\int_{0}^{2 \pi} \int_{0}^{\infty} I_{0} \exp \left(\frac{-\rho^{2}}{2 \sigma^{2}}\right) \rho d \phi d \rho=2 \pi \sigma^{2} I_{0}
$$

que depende da potência do laser. Assim, com as condições de Abbe ${ }^{[16]}$ que estabelecem que:

$$
\rho=f \operatorname{sen} \theta,
$$

Sendo $f$ a distância focal da objetiva, que usamos na Equação 2.46

$$
d P=\frac{P}{2 \pi \sigma^{2}} \exp \left(\frac{-f^{2} \operatorname{sen} \theta^{2}}{2 \sigma^{2}}\right) d \phi f^{2} \operatorname{sen} \theta \cos \theta d \theta
$$

Agora, podemos expressar o potencial local, ou seja, o qual a microesfera é submetida, onde integramos nos limites de $0<\theta<\theta_{M}, 0<\phi<2 \pi$. Nesse caso, $\theta_{M}$ representa o ângulo máximo de refração (Apêndice B), onde, para ângulos maiores, o feixe é totalmente refletido pela lâmina. Desta maneira, chegamos à expressão final da potência em função da distância focal e do ângulo de incidência máximo do feixe na lâmina ou recipiente da amostra.

$$
P_{\text {local }}=P\left[1-\exp \left(\frac{-f^{2} \operatorname{sen} \theta_{M}{ }^{2}}{2 \sigma^{2}}\right)\right]
$$

Logo, podemos escrever a potência total em função da posição. 


$$
d P=\frac{\frac{P}{2 \pi \sigma^{2}} \exp \left(\frac{-f^{2} \operatorname{sen} \theta^{2}}{2 \sigma^{2}}\right) T(\theta, \phi) d \phi f^{2} \operatorname{sen} \theta \cos \theta d \theta}{\left[1-\exp \left(\frac{-f^{2} \operatorname{sen} \theta_{M}{ }^{2}}{2 \sigma^{2}}\right)\right]}
$$

De posse desses resultados, podemos calcular a força total do feixe sobre a microesfera:

$$
\vec{F}=\frac{n_{2}}{c} \int_{0}^{2 \pi} \int_{0}^{\theta_{M}}\left[\operatorname{Re}\left(Q_{z}\right) \hat{z}^{\prime \prime}+\operatorname{Im}\left(Q_{z}\right) \hat{y}^{\prime \prime}\right] d P
$$

Onde, para caso real, temos que considerar o coeficiente de transferência $T(\theta, \phi)$, que expressa a porcentagem perdida de potência da luz ao atravessar o vidro.

\subsection{Teorias eletromagnéticas de (Lorenz - Mie) para $\lambda \cong d$}

\subsubsection{A formulação das equações de ondas eletromagnéticas e algumas probabilidades}

A teoria para explicar o efeito de uma onda eletromagnética sobre uma partícula, para qualquer comprimento, incluído o comprimento da partícula $d \cong \lambda$, só foi desenvolvida entre o final do século XIX e começo do século XX, por Lorenz. No entanto, esse trabalho foi amplamente ignorado por ter sido escrito em dinamarquês e por não usar o formalismo de Maxwell, que passou a ser padrão a partir de 1864, sendo apenas reconhecido então em 1980 por estudiosos da época ${ }^{[1]}$. O trabalho lido pela maior parte da comunidade científica foi o do físico alemão Gustavo Mie, que o desenvolveu para explicar a absorção e o espalhamento de partículas coloidais de ouro suspensas em água. Como essa é uma teoria geral, o motivo de usarmos 3 teorias para 3 situações diferentes se deve ao fato de que a teoria de Mie é muito complexa, sendo que, no limite $d \ll \lambda$, e $d \gg \lambda$, ela possui as mesma previsões que outras duas teorias, então, por facilidade, utilizamos a teoria de Mie somente em regimes onde as duas outras teorias não são suficientes. Esse regime corresponde à região entre os dois limites. No entanto, a teoria de Lorenz-Mie só pode ser aplicada para ondas planas.

Com o advento do laser e com a possibilidade de luz altamente focalizada, foi necessária a expansão da teoria de ondas esféricas, ou a chamada teoria GLMT, teoria essa que apresenta grande dificuldade matemática, já sendo desenvolvida em outras teses e artigos. Nessa dissertação, não mostraremos todo seu desenvolvimento, utilizando-nos de apenas uma parte. Sua explicação completa pode ser consultada nas referências ${ }^{[1][13][16]}$.que sao as outras teses artigos citados anteriormente.

Vamos considerar uma partícula com tamanho e forma definidos, nesse caso, uma esfera, iluminada por uma onda eletromagnética. Considerando também que a partícula possui seu próprio campo eletromagnético vindo da sua densidade eletrônica $\left(\vec{E}_{\text {int }}, \vec{H}_{\text {int }}\right)$, e, que a 
luz é descrita como uma superposição de campos eletromagnéticos, sendo esse os incidentes $\left(\vec{E}_{i}, \vec{H}_{i}\right)$. Os campos incidentes, depois de colidirem com a esfera, se transformam em campos espalhados $\left(\vec{E}_{e}, \vec{H}_{e}\right)$, então, utilizando o princípio de conservação de carga, postulamos as seguintes relações, onde dos campos sobrepostos $\left(\vec{E}_{1} \vec{H}_{1}\right)$ que são:

$$
\begin{aligned}
& \overrightarrow{E_{1}}=\vec{E}_{e}+\vec{E}_{i} \\
& \vec{H}_{1}=\vec{H}_{e}+\vec{H}_{i}
\end{aligned}
$$

onde os campos totais são $\vec{E}=\vec{E}_{1}+\vec{E}_{\text {int }}, \vec{H}=\vec{H}_{1}+\vec{H}_{\text {int }}$.

Considerando que cada um desses campos descritos é tridimensional e descrito em coordenadas esféricas $(\vec{r}, \theta, \phi)$ com $\vec{k}$ sendo o vetor de onda apropriada do meio, nesse caso, a água. Onde os campos eletromagnéticos satisfazem as relações de Maxwell:

$$
\begin{array}{r}
\nabla \cdot \vec{E}=0 \\
\nabla \cdot \vec{H}=0 \\
\nabla \times \vec{E}=i k Z \vec{H} \\
\nabla \times \vec{H}=-i k \vec{E} / Z
\end{array}
$$

E $Z$ representa a impedância da meio definida como $\sqrt{\mu / \varepsilon}$, em que $\mu$ e $\varepsilon$ são contínuos.

Dessa maneira, aplicando rotacional nas equações:

$$
\begin{gathered}
\nabla \times(\nabla \times \vec{E})=i k Z \nabla \times \vec{H}=k^{2} \vec{E} \\
\nabla \times(\nabla \times \vec{H})=-i\left(\frac{k}{Z}\right) \nabla \times \vec{E}=k^{2} \vec{H}
\end{gathered}
$$

Usando a identidade vetorial, nas equações:

$$
\nabla \times(\nabla \times \vec{A})=\nabla(\nabla \cdot \vec{A})-\nabla^{2} \vec{A}
$$

onde $\vec{A}$ é um campo vetorial qualquer. Na equação acima chegamos à equação de Helmholtz:

$$
\nabla^{2} \vec{E}+k^{2} \vec{E}=0 \quad \nabla^{2} \vec{H}+k^{2} \vec{H}=0
$$

onde o campo eletromagnético da luz será um qualquer que satisfaça as condições de onda acima. 
Logo, aplicaremos a condição de onda transversais, em que temos que os campos satisfazem $\vec{r} \cdot \vec{L}=0=\vec{r} \cdot \vec{E}$, havendo duas condições: os campos de ondas transversais magnéticos (TM) e elétricos (TE).

$$
\begin{array}{ll}
\vec{E}^{T E}=\vec{L} \psi & \vec{H}^{T E}=\frac{-i}{k Z} \nabla \times \vec{L} \psi \\
\vec{H}^{T M}=\vec{L} \psi & \vec{E}^{T M}=\frac{i Z}{k} \nabla \times \vec{L} \psi
\end{array}
$$

Podemos escrever e separa as soluções em

$$
\begin{array}{r}
\psi_{n m}^{(T M)}=\sum_{n, m} z_{n}^{(1)}(k r) Y_{n m}(\phi, \theta) \\
\psi_{n m}^{(T E)}=\sum_{n, m} z_{n}^{2}(k r) Y_{n m}(\phi, \theta) \\
\psi_{n m}=\sum_{n, m}\left(A_{n m} z_{n}^{1}(k r)+B_{n m} z_{n}^{2}(k r)\right) Y_{n m}(\phi, \theta)
\end{array}
$$

onde $z_{n}^{i}(k r)$ pode ser qualquer uma das funções de Bessel. Nesse caso, considerando as partículas como esferas, que podem ser tanto esféricas normais $j_{n}(k r)$, a qual apresenta como função esférica $y_{n}(k r)$ ou a combinação linear delas, as funções de Hankel $h_{n}^{1}(k r), h_{n}^{2}(k r)$, que serão definidas adiante, dependerão das condições do contorno. Por exemplo, para condição de $k r \rightarrow 0$, as funções de Hankel divergem para o campo no interior da esfera e, desta maneira, somente as equações de Bessel esféricas são permitidas. Portanto, definido o campo em relações as duas partes da função de onda teremos, onde os campos elétricos e magnéticos são escritos como:

$$
\begin{aligned}
& \vec{E}_{n m}^{(T E)}=\vec{L} \psi_{n m}^{(T E)}=z_{n}^{(2)}(k r) \vec{L} Y_{n m}(\phi, \theta) \\
& \vec{H}_{n m}^{(T E)}=\frac{-i}{k Z} \nabla \times\left[z_{k r}^{(2)} \vec{L} Y_{n m}(\phi, \theta)\right] \\
& \vec{E}_{n m}^{(T M)}=\vec{L} \psi_{n m}^{(T M)}=z_{n}^{(1)}(k r) \vec{L} Y_{n m}(\phi, \theta) \\
& \vec{H}_{n m}^{(T M)}=\frac{i Z}{K} \nabla \times\left[z_{k r}^{(1)} \vec{L} Y_{n m}(\phi, \theta)\right]
\end{aligned}
$$

Esses conjuntos de equações formam um conjunto completo de equações de Maxwell em uma região livre de fontes. Sendo que podemos escrever $\vec{L} Y_{n m}$ como o vetor harmônico esférico.

$$
\vec{X}_{n m}(\theta, \phi)=\frac{1}{\sqrt{n(n+1)}} \vec{L} Y_{n m}(\theta, \phi)
$$

Sendo esse vetor ortogonal ${ }^{[1]}$, a aplicação dessas definições ao problema da esfera com campo eletomagnético (EM) incidente gera campos que podem ser escritos como:

$$
\vec{E}=E_{0} \sum_{n, m}\left[\frac{i}{k} A_{n m} \nabla \times z_{n}(k r) \vec{X}_{n m}(\theta, \phi)+B_{n m} z_{n}(k r) \vec{X}_{n m}(\theta, \phi)\right.
$$




$$
\vec{H}=H_{0} \sum_{n m}\left[\frac{-i}{k} B_{n m} \nabla \times z_{n}(k r) \vec{X}_{n m}(\theta, \phi)+A_{n m} z_{n}(k r) \vec{X}_{n m}(\theta, \phi)\right.
$$

onde as amplitudes estão relacionadas pela impedância do meio, $H_{0}=Z E_{0}$, e $A_{n m}$ e $B_{n m}$ que são as amplitudes das diversas ondas parciais, além de especificar a quantidade de multipolos. Para determinar esses coeficientes de espaço, multiplicamos por $\vec{r}$, onde temos que:

$$
\begin{aligned}
& \vec{E} \cdot \vec{r}=E_{0} \sum_{n, m}\left[\frac{i}{k} A_{n m} \vec{r} \cdot \nabla \times z_{n}(k r) \vec{X}_{n m}(\theta, \phi)+B_{n m} \vec{r} \cdot z_{n}(k r) \vec{X}_{n m}(\theta, \phi)\right] \\
& \vec{H} \cdot \vec{r}=H_{0} \sum_{n m}\left[\frac{-i}{k} B_{n m} \vec{r} \cdot \nabla \times z_{n}(k r) \vec{X}_{n m}(\theta, \phi)+A_{n m} \vec{r} \cdot z_{n}(k r) \vec{X}_{n m}(\theta, \phi)\right]
\end{aligned}
$$

onde temos a relação $\vec{r} \cdot X_{n m}(\theta, \phi) \approx \vec{r} \cdot \vec{L}=0$, o que nos deixa com:

$$
\vec{r} \cdot \nabla \times z_{n}(k r) X_{n m}(\theta, \phi)=\vec{r} \cdot\left(\nabla z_{n}(k r) X_{n m}(\theta, \phi)\right)+z_{n}(k r) \vec{r} \cdot\left(\nabla \times X_{n m}(\theta, \phi)\right)
$$

O primeiro termo da equação é nulo, pois ele só depende da função radial $z_{n}(k r)$, logo, o produto escalar geral é um componente perpendicular, portanto, zero. Assim, nos resta apenas a outra parte da equação, onde, usando as relações $\vec{r} \cdot(\nabla \times \vec{L})=i L^{2}$ e $L^{2} Y_{n m}=n(n+$ 1) $Y_{n m}(\phi, \theta)$, temos que:

$$
\vec{r} \cdot \nabla \times z_{n}(k r) X_{n m}(\theta, \phi)=z_{n}(k r) i \sqrt{n(n+1)} Y_{n m}(\phi, \theta)
$$

Lembrando que, o harmônico esférico não depende da variável radial, então, a componente radial do produto escalar do componente esférico é nulo, como sua derivada. Desta forma, podemos escrever a equação como:

$$
\begin{gathered}
\vec{E} \cdot \vec{r}=-E_{0} \sum_{n, m} \frac{1}{k} A_{n m} \sqrt{n(n+1)} z_{n}(k r) Y_{n m}(\theta, \phi) \\
\vec{H} \cdot \vec{r}=H_{0} \sum_{n, m} \frac{1}{k} B_{n m} \sqrt{n(n+1)} z_{n}(k r) Y_{n m}(\theta, \phi)
\end{gathered}
$$

Multiplicando por $Y_{n m}^{*}(\theta, \phi)$ e trocando as variáveis n por n' e integrando em relação ao ângulo sólido $d \Omega$ :

$$
\begin{aligned}
\int Y_{n m}^{*}(\theta, \phi) \vec{E} & \cdot \vec{r} d \Omega= \\
& =-E_{0} \sum_{n, m} \frac{1}{k} A_{n^{\prime} m^{\prime}} \sqrt{n^{\prime}\left(n^{\prime}+1\right)} z_{n^{\prime}}(k r) \int Y_{n m}^{*}(\theta, \phi) Y_{n m}(\theta, \phi) d \Omega \\
& =-E_{0} \sum_{n, m} \frac{1}{k} A_{n^{\prime} m^{\prime}} \sqrt{n^{\prime}\left(n^{\prime}+1\right)} z_{n^{\prime}}(k r) \delta_{\mathrm{n}, \mathrm{n}^{\prime}} \delta_{\mathrm{m}, \mathrm{m}^{\prime}} \\
& =-E_{0} \frac{1}{k} A_{n m} \sqrt{n(n+1)} z_{n}(k r)
\end{aligned}
$$




$$
\int Y_{n m}^{*}(\theta, \phi) \vec{H} \cdot \vec{r} d \Omega=H_{0} \frac{1}{k} B_{n m} \sqrt{n(n+1)} z_{n}(k r)
$$

Logo, podemos determinar os componentes múltiplos de campo eletromagnético qualquer com as relações:

$$
\begin{aligned}
& A_{n m} Z_{n}(k r)=\frac{-k}{E_{0} \sqrt{n(n+1)}} \int Y_{n m}^{*}(\theta, \phi) \vec{E} \cdot \vec{r} d \Omega \\
& B_{n m} z_{n}(k r)=\frac{k}{H_{0} \sqrt{n(n+1)}} \int Y_{n m}^{*}(\theta, \phi) \vec{H} \cdot \vec{r} d \Omega
\end{aligned}
$$

Sendo essas equações utilizadas para determinar os coeficientes $A_{n m}, B_{n m}$, no entanto, são necessárias as expressões para campos que não depende destes coeficientes. Na Seção 2.4.2, apresentaremos os coeficientes do campo de incidência, que, a partir do campo calculado para essa onda e das Equações 2.82 e 2.83, é possível chegar esses coeficientes. As obtenções dos coeficientes dos demais campos envolvidos na força eletromagnética utilizaram outro processo para sua determinação e estão apresentados no Apêndice A.

2.4.2 Determinação das 3 ondas eletromagnéticas e apresentação de seus coeficientes de contorno

Utilizando as Equações 2.72, 2.73, 2.80 e 2.81 podemos escrever cada campo, o espalhado, o interno da partícula e o incidente, como funções com coeficientes múltiplos, conforme explicado na Seç̧ão 2.4.1, onde cada um terá seu próprio coeficiente de contorno. Além das funções radiais serem descritas como funções esféricas de Bessel $j_{n}$ e Hankel $h_{n}^{1}$, cujas definições estão descritas na literatura ${ }^{[13][14]}$. Inicialmente utilizaremos as equações para campo incidente, tendo:

$$
\begin{aligned}
& \vec{E}_{l}=E_{0} \sum_{n, m}\left[\frac{i}{k_{1}} G_{n m}^{T M} \nabla \times j_{n}\left(k_{1} r\right) \vec{X}_{n m}(\theta, \phi)+G_{n m}^{T E} j_{n}\left(k_{1} r\right) \vec{X}_{n m}(\theta, \phi)\right. \\
& \vec{H}_{l}=\frac{E_{0}}{z_{1}} \sum_{n, m}\left[\frac{-i}{k_{1}} G_{n m}^{T E} \nabla \times j_{n}\left(k_{1} r\right) \vec{X}_{n m}(\theta, \phi)+G_{n m}^{T M} j_{n}\left(k_{1} r\right) \vec{X}_{n m}(\theta, \phi)\right]
\end{aligned}
$$

Para o campo depois de espalhado, temos:

$$
\begin{gathered}
\vec{E}_{e}=E_{0} \sum_{n, m}\left[\frac{i}{k_{1}} a_{n m} \nabla \times h_{n}^{1}\left(k_{1} r\right) \vec{X}_{n m}(\theta, \phi)+b_{n m} h_{n}^{1}\left(k_{1} r\right) \vec{X}_{n m}(\theta, \phi)\right] \\
\vec{H}_{e}=\frac{H_{0}}{z_{1}} \sum_{n, m}\left[\frac{-i}{k_{1}} b_{n m} \nabla \times h_{n}^{1}\left(k_{1} r\right) \vec{X}_{n m}(\theta, \phi)+a_{n m} h_{n}^{1}\left(k_{1} r\right) \vec{X}_{n m}(\theta, \phi)\right]
\end{gathered}
$$

E, por último, as ondas do campo interno da partícula, onde, para que tenhamos um valor finito na origem adotamos $k_{2}=2 \pi n_{1} / \lambda$, sendo o número de onda dentro da esfera, e o índice relativo $M=\frac{n_{1}}{n_{2}}$. 


$$
\begin{aligned}
& \vec{E}_{\text {int }}=E_{0} \sum_{n, m}\left[\frac{i}{k_{2}} c_{n m} \nabla \times j_{n}\left(k_{2} r\right) \vec{X}_{n m}(\theta, \phi)+d_{n m} j_{n}\left(k_{2} r\right) \vec{X}_{n m}(\theta, \phi)\right] \\
& \vec{H}_{\text {int }}=\frac{E_{0}}{z_{2}} \sum_{n, m}\left[\frac{-i}{k_{2}} d_{n m} \nabla \times j_{n}\left(k_{2} r\right) \vec{X}_{n m}(\theta, \phi)+c_{n m} j_{n}\left(k_{2} r\right) \vec{X}_{n m}(\theta, \phi)\right]
\end{aligned}
$$

As equações dos campos dependem das equações de Bessel e da equação de Henkel, em sua forma esférica, as quais são expressas por $^{[14],[15]}$

$$
\begin{gathered}
j_{n}(k r)=2^{n}(k r)^{n} \sum_{s=0}^{\infty} \frac{(-1)^{s}(s+n) !}{s !(2 s+2 n+1) !}(k r)^{2 s} \text { onde para }=0 \rightarrow j_{0}(k r)= \\
\sum_{s=0}^{\infty} \frac{(-1)^{s}}{(2 s+1) !}(k r)^{2 s}=\frac{\operatorname{sen}(k r)}{k r}
\end{gathered}
$$

As equações de Bessel esférica obedecem à seguinte propriedade ${ }^{[14][15]}$ :

$$
\begin{gathered}
j_{n}(k r)=(-k r)^{n}\left(\frac{1}{k r} \frac{d}{d r}\right)^{n} \frac{\operatorname{sen} k r}{k r} \\
y_{n}(k r)=-(-k r)^{n}\left(\frac{1}{x} \frac{d}{d x}\right)^{n} \frac{\cos x}{x}
\end{gathered}
$$

onde $y_{n}$ é a equação esférica de Bessel em sua segunda forma, utilizando-a nessa teoria para determinar a equação de Henkel define-se:

$$
\begin{aligned}
& h_{n}^{1}(k r)=j_{n}(k r)+i y_{n}(k r) \\
& h_{n}^{2}(k r)=j_{n}(k r)-i y_{n}(k r)
\end{aligned}
$$

Assumimos a equação de Henkel como segunda na onda de espalhamento, pois consideramos que no espalhamento, a onda se divida nos dois tipos, logo, sua solução seria uma combinação linear entre as ondas esféricas de Bessel. Sendo que as equações de Henkel estão na sua forma assintóptica $(k r \rightarrow \infty)$,

$$
\begin{gathered}
h_{n}^{1}(k r) \sim \frac{(-i)^{n} e^{i k r}}{i k r} \\
\operatorname{com} r \gg n^{2}, \\
h_{n}^{2}(k r) \sim-\frac{i^{n} e^{-i k r}}{i k r} \quad(2.95
\end{gathered}
$$

Além disso, introduzindo outras duas formas da equação de Bessel, onde a primeira é a forma assintóptica, com:

$$
j_{n}=\frac{(-i)^{n+1} e^{i k r}}{2 k r}+\frac{(i)^{n+1} e^{-i k r}}{2 k r}
$$

Por fim, a funções de Riccati-Bessel,

$$
\psi_{n}=\rho j_{n}(\rho) \quad \xi_{n}(\rho)=\rho h_{n}^{(1)}(\rho)
$$

estando essas funções e suas sucessivas deduções desenvolvidas no Apêndice A. 
Desta maneira, obtemos os coeficientes $a_{n}, b_{n}$ em função de $a_{n m}, b_{n m}$ onde $x=k_{2} d$, que são chamados de Coeficientes de Mie:

$$
\begin{aligned}
& -a_{n}=\frac{a_{n m}}{G_{n m}^{T M}}=\frac{M \psi_{n}(M x) \psi_{n}{ }^{\prime}(x)-\psi_{n}^{\prime}(M x) \psi_{n}(x)}{\psi_{n}^{\prime}(M x) \xi_{n}(x)-M \psi_{n}(M x) \xi_{n}^{\prime}(x)} \\
& -b_{n}=\frac{b_{n m}}{G_{n m}^{T E}}=\frac{M \psi_{n}^{\prime}(M x) \psi_{n}(x)-\psi_{n}(M x) \psi_{n}^{\prime}(x)}{\psi_{n}(M x) \xi_{n}^{\prime}(x)-M \psi_{n}^{\prime}(M x) \xi_{n}(x)} \\
& -c_{n}=\frac{c_{n m}}{G_{n m}^{T E}}=\frac{\psi_{n}(x) \xi_{n}^{\prime}(x)-\psi_{n}^{\prime}(x) \xi_{n}(x)}{\psi_{n}^{\prime}(M x) \xi_{n}(x)-M \psi_{n}(M x) \xi_{n}^{\prime}(x)} \\
& -d_{n}=\frac{d_{n m}}{G_{n m}^{T E}}=\frac{\psi_{n}^{\prime}(x) \xi_{n}(x)-\psi_{n}(x) \xi_{n}(x)}{\psi_{n}(M x) \xi_{n}^{\prime}(x)-M \psi_{n}^{\prime}(M x) \xi_{n}(x)}
\end{aligned}
$$

Essas equações características são independentes do feixe incidente. Os coeficientes do campo espalhado e do campo no interior da partícula são independes do índice M devido á simetria esférica do espalhador. Caso a esfera fosse deformada, os modos azimutais não seriam mais degenerados. Definimos os coeficientes negativos por definição na literatura ${ }^{[1]}$. Faz-se necessário abordar também o modo de domínio dos coeficientes, onde, se a condição $\frac{\xi_{n}^{\prime}(x)}{\xi_{n}(x)}=\frac{\psi_{n}^{\prime}(M x)}{M \psi_{n}(M x)}$ for satisfeita então temos que $d_{n}$ e $a_{n}$ são dominantes, se $\frac{\xi_{n}^{\prime}(x)}{\xi_{n}(x)}=\frac{M \psi_{n}^{\prime}(M x)}{\psi_{n}(M x)}$ então $b_{n}$ e $c_{n}$. As frequências que duas condições são satisfeitas constitui uma condição chamada de MDR (do inglês, morphology dependent resonance).

E, por último, faltou determinar as funções $G_{n m}^{T M}, G_{n m}^{T E}$. Escolhemos não realizar essa dedução por sua extensão e, também, pois a dedução completa pode ser encontrada em outras referências ${ }^{[1]][6][12][16]}$. Uilizamos essas equações, juntamente com os coeficientes de Mie, nas Equações 2.84-2.87 pra chegarmos nas expresses dos campos E, H:

$$
\begin{gathered}
G_{n m}^{T M}=4 \pi i^{n-m} e^{-i m \phi_{0}} i k f e^{-i k f} \sqrt{\frac{n_{0}}{n_{3}}} \sqrt{\frac{2 n+1(n-m) !}{4 \pi n(n+1)(n+m) !}}\left[I_{1}+I_{2}\right] \\
G_{n m}^{T E}=-4 \pi i^{n-m} e^{-i m \phi_{0}} i k f e^{-i k f} \sqrt{\frac{n_{0}}{n_{3}}} \sqrt{\frac{2 n+1(n-m) !}{4 \pi n(n+1)(n+m) !}}\left[I_{3}+I_{4}\right] \\
\int_{0}^{\theta_{\max }} d \alpha \sqrt{\cos \alpha^{\prime \prime \prime}} e^{-\frac{f^{2} \operatorname{sen}^{2} \alpha^{\prime \prime \prime}}{\omega_{0}^{2}}} e^{-i z_{0} \cos \alpha^{\prime \prime \prime}} m^{2} P_{n}^{m}\left(\cos \alpha^{\prime \prime \prime}\right) \frac{J_{m}\left(k p_{0} \operatorname{sen} \alpha^{\prime \prime \prime}\right)}{k p_{0} \operatorname{sen} \alpha^{\prime \prime \prime}} \\
\left.\operatorname{sen}^{2} \alpha P^{\prime m}\left(\cos \alpha^{\prime \prime \prime}\right) J^{\prime}{ }_{m}\left(k \rho_{0} \operatorname{sen} \alpha^{\prime \prime \prime}\right)\right\} \cos \phi_{0} \\
I_{2}=\int_{0}^{\theta_{\max }} d \alpha \sqrt{\cos \alpha^{\prime \prime \prime}} e^{-\frac{f^{2} \operatorname{sen}^{2} \alpha^{\prime \prime \prime}}{\omega_{0}^{2}}} e^{-i z_{0} \cos \alpha^{\prime \prime \prime}} i m P_{n}^{m}\left(\cos \alpha^{\prime \prime \prime}\right) J_{m}^{\prime}\left(k \rho_{0} \operatorname{sen} \alpha^{\prime \prime \prime}\right)- \\
\left.\operatorname{sen}^{2} \alpha^{\prime \prime \prime}\left(\cos \alpha^{\prime \prime \prime}\right) \frac{J_{m}\left(k p_{0} \operatorname{sen} \alpha\right)}{k p_{0} \operatorname{sen} \alpha^{\prime \prime \prime}}\right\} \operatorname{sen} \phi_{0}
\end{gathered}
$$




$$
\begin{aligned}
& I_{3}=\int_{0}^{\theta_{\max }} d \alpha \sqrt{\cos \alpha^{\prime \prime \prime}} e^{-\frac{f^{2} \operatorname{sen}^{2} \alpha^{\prime \prime \prime}}{\omega_{0}^{2}}} e^{-i z_{0} \cos \alpha^{\prime \prime}} m^{2 P_{n}^{m}}\left(\cos \alpha^{\prime \prime \prime}\right) \frac{J_{m}\left(k p_{0} \operatorname{sen} \alpha^{\prime \prime \prime}\right)}{k p_{0} \operatorname{sen} \alpha^{\prime \prime}}- \\
& \left.\operatorname{sen}^{2} \alpha P_{n}^{\prime m}\left(\cos \alpha^{\prime \prime \prime \prime}\right) J_{m}^{\prime}\left(k \rho_{0} \operatorname{sen} \alpha^{\prime \prime \prime \prime}\right)\right\} \operatorname{sen} \phi_{0}
\end{aligned}
$$

$$
\begin{aligned}
& I_{4}=\int_{0}^{\theta_{\max }} d \alpha \sqrt{\cos \alpha^{\prime \prime \prime}} e^{-\frac{f^{2} \operatorname{sen}^{2} \alpha^{\prime \prime \prime}}{\omega_{0}^{2}}} e^{-i z_{0} \cos \alpha^{\prime \prime \prime}} i m P_{n}^{m}\left(\cos \alpha^{\prime \prime \prime}\right) J_{m}^{\prime}\left(k \rho_{0} \operatorname{sen} \alpha^{\prime \prime \prime}\right)- \\
& \left.\operatorname{sen}^{2} \alpha\left(\cos \alpha^{\prime \prime \prime}\right) \frac{J_{m}\left(k p_{0} \operatorname{sen} \alpha^{\prime \prime \prime}\right)}{k p_{0} \operatorname{sen} \alpha^{\prime \prime \prime}}\right\} \cos \phi_{0}
\end{aligned}
$$

Como podemos perceber, foram introduzidas novas constantes e variáveis, sendo a primeira $n_{3}$, que representa o índice de refração do vidro da lâmina, e $n_{0}$, o índice de refração do ar, água ou óleo, ou seja, do meio onde o feixe entra ao sair da objetiva. Podemos utilizar água ou óleo para realização dos experimentos e devido acoplamento da direção do laser. Devido à caracteróstica da objetiva que tínhamos em nosso laboratório, utilizamos óleo de imersão de índice similar ao índice de refração do vidro utilizado para a construção das lamínulas de microscopia. Também, $f$ representa a distância focal da objetiva usada e $\omega_{0}$ a largura do feixe na entrada da objetiva.

Para esses coeficientes, há uma mudança de coordenadas cilíndricas dada pela Equação 2.104, sendo a função integrada de um ângulo 0 até $\theta_{\max }$ de refração, análogo ao ângulo $\theta_{M}$ das seções anteriores e do Apêndice $\mathrm{B}$, mas diferente nesse caso, onde seu valor é $\theta_{\max }=\operatorname{sen}^{-1}\left(\frac{N A}{n_{2}}\right)$, com NA representando a abertura numérica da objetiva usada.

$$
\begin{gathered}
\rho \cos \varphi=r \operatorname{sen} \theta \cos \phi-\rho_{0} \cos \phi_{0} \\
\rho \operatorname{sen} \varphi=r \operatorname{sen} \theta \operatorname{sen} \phi-\rho_{0} \operatorname{sen} \phi_{0} \\
z=r \cos \theta-z_{0}
\end{gathered}
$$

Mudamos, também, as variáveis de integração para distinguir entre a coordenadas do sistema esférico com as da objetiva de $(\theta, \phi) \rightarrow\left(\alpha^{\prime \prime \prime}, \beta^{\prime \prime \prime}\right)$. Na Figura 2.9 representamos as ondas que saem da objetiva, atravessando os meios e sendo refratadas, e, na Figura 2.10 representamos a mudança de coordenada do meio esférico para cilíndrico do feixe de laser incidente. Sendo que o campo está em função $\beta^{\prime \prime \prime}$ e as coordenadas em relação a $\beta^{\prime \prime}$ são eliminadas por uma transformação não demonstrada no presente trabalho ${ }^{[1]}$. 


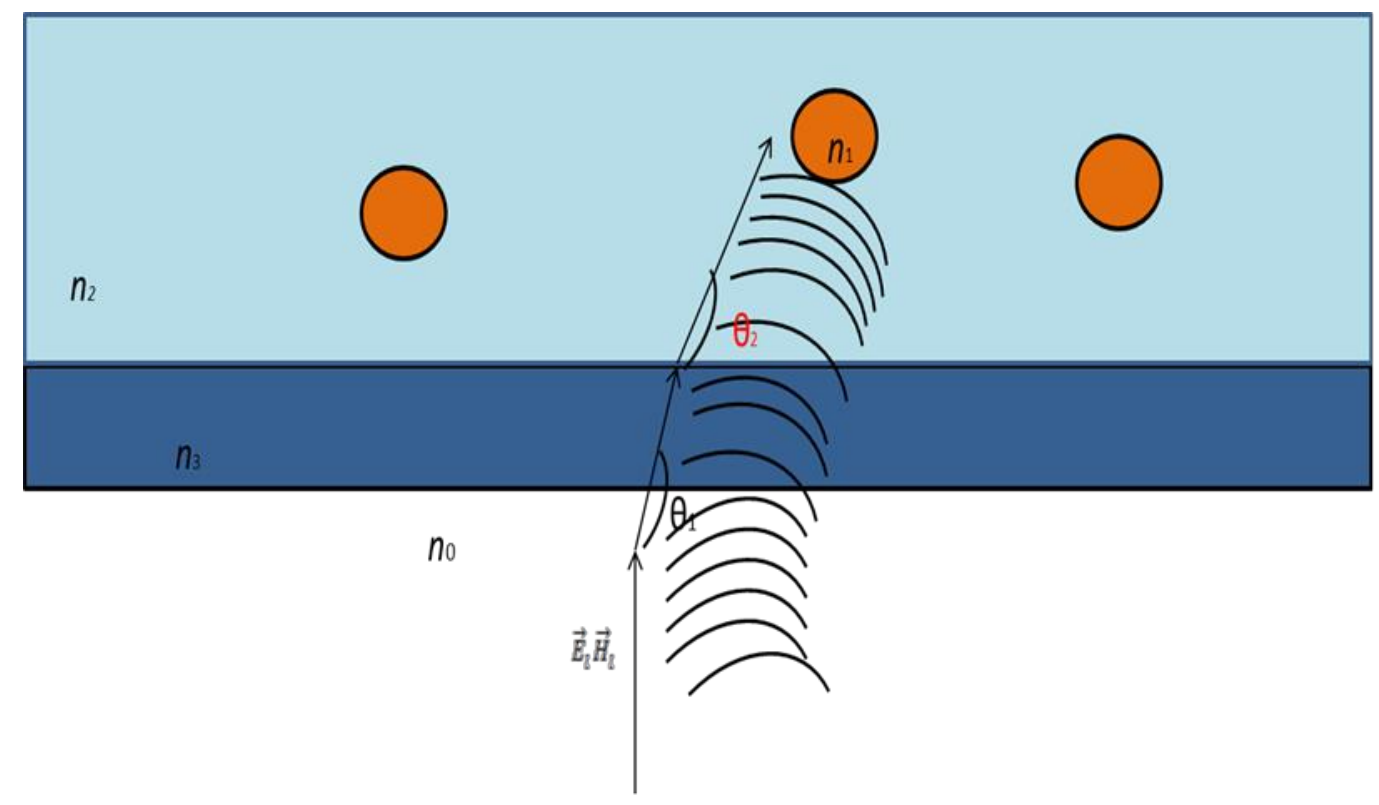

Figura 2.9: Propagação das ondas que saem da objetiva.

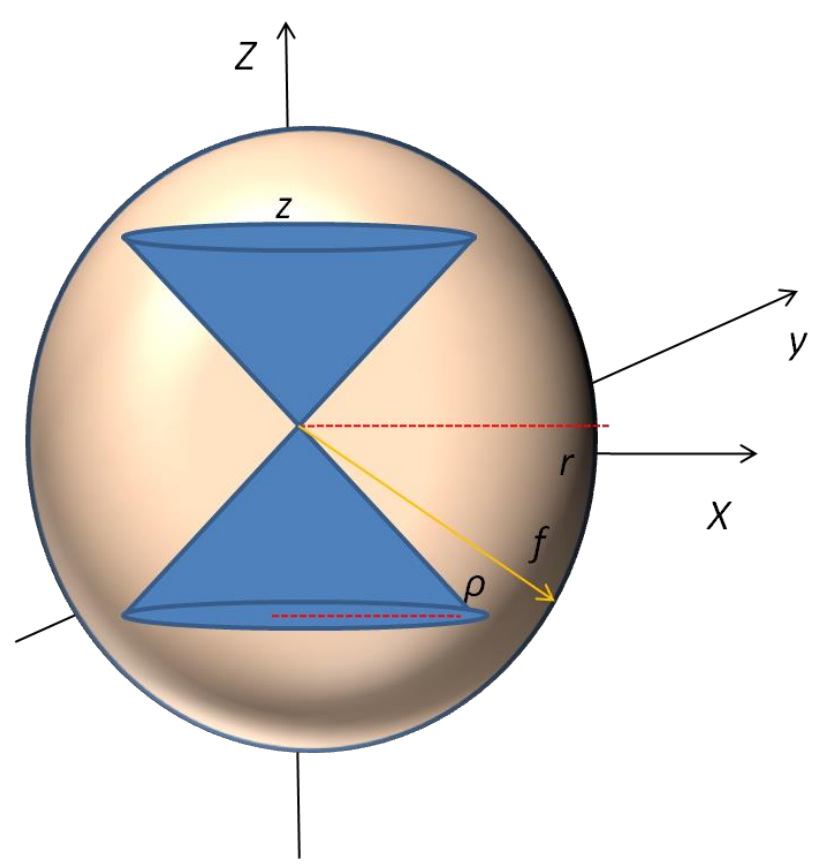

Figura 2.10: Descrição da mudança de variáveis esféricas para cônicas da onda incidente

Outro fenômeno a relatar que altera as Equações 2.98 à 2.103 que é o deslocamento focal. Como no anterior só irei apresentar as equações finais, onde sua dedução completa se encontra em outras referências ${ }^{[1]}$. 
Nossa objetiva encontra-se imersa em óleo, o qual possui índice de refração similar ao da lamínula. No entanto, o conjunto óleo/lamínula/água provoca uma profundidade aparente chamada de deslocamento focal $d f$. Esse deslocamento é dado por ${ }^{[19][20]}$

$$
d f=\frac{\Delta f}{1-\frac{n_{2}}{n_{3}} \sqrt{1-\left(\frac{L}{2 f}\right)^{2}\left[\left(\frac{n_{2}}{n_{2}}\right)^{2}-1\right]}}
$$

Esse efeito introduz mudanças nas Equações 2.110 e 2.111, cujas deduções podem ser encontradas na literatura ${ }^{[1][21],}$

$$
G_{n m}^{T M}=4 \pi i^{n-m} e^{-i m \phi_{0}} i k f e^{-i k f} \sqrt{\frac{n_{0} n_{2}}{n_{3}}} \sqrt{\frac{2 n+1(n-m) !}{4 \pi n(n+1)(n+m) !}}\left[I^{\prime}{ }_{1} \cos \phi_{0}+i m I^{\prime}{ }_{2} \operatorname{sen} \phi_{0}\right]
$$

$$
G_{n m}^{T E}=-4 \pi i^{n-m} e^{-i m \phi_{0}} i k f e^{-i k f} \sqrt{\frac{n_{0} n_{2}}{n_{3}}} \sqrt{\frac{2 n+1(n-m) !}{4 \pi n(n+1)(n+m) !}}\left[I^{\prime}{ }_{1} \operatorname{sen} \phi_{0}-i m I^{\prime}{ }_{2} \operatorname{sen} \phi_{0}\right]
$$

$$
\int_{0}^{\theta_{\max }} d \alpha \sqrt{\cos \alpha^{\prime \prime \prime}} e^{-\frac{f^{2} \operatorname{sen}^{2} \alpha^{\prime \prime \prime}}{\omega_{0}^{2}}} e^{i k_{2}\left(d-z_{0}\right) \cos \alpha_{2}{ }^{\prime \prime}} e^{-i d k \cos \alpha^{\prime \prime \prime}}\left(t^{s} m^{2} P_{n}^{m}\left(\cos \alpha^{\prime \prime \prime}\right) \frac{J_{m}\left(k p_{0} \operatorname{sen} \alpha^{\prime \prime \prime}\right)}{k p_{0} \operatorname{sen} \alpha^{\prime \prime \prime}}-\right.
$$

$$
t^{p} \operatorname{sen}^{2} \alpha P_{n}^{\prime m}\left(\cos \alpha^{\prime \prime \prime}\right) J_{m}^{\prime}\left(k \rho_{0} \operatorname{sen} \alpha^{\prime \prime \prime}\right)
$$

$$
\begin{gathered}
\int_{0}^{\theta_{\max }} d \alpha \sqrt{\cos \alpha^{\prime \prime \prime}} e^{-\frac{f^{2} \operatorname{sen}^{2} \alpha^{\prime \prime \prime}}{\omega_{0}^{2}}} e^{i k_{2}\left(d-z_{0}\right) \cos \alpha_{2}{ }^{\prime \prime \prime}} e^{-i d k \cos \alpha^{\prime \prime \prime}}\left(t^{s} P_{n}^{m}\left(\cos \alpha^{\prime \prime \prime}\right) J_{m}^{\prime}\left(k \rho_{0} \operatorname{sen} \alpha^{\prime \prime \prime}\right)-\right. \\
\left.\operatorname{sen}^{2} \alpha^{\prime \prime \prime} t^{p}\left(\cos \alpha^{\prime \prime \prime}\right) \frac{J_{m}\left(k p_{0} \operatorname{sen} \alpha\right)}{k p_{0} \operatorname{sen} \alpha^{\prime \prime}}\right)
\end{gathered}
$$

Onde, $t^{s}, t^{p}$ são equações referentes a reflexão da onda dados por:

$$
\begin{array}{r}
t^{s}=\frac{2 \cos \theta}{\cos \theta+\left(\frac{n_{2}}{n_{3}}\right) \cos \theta_{2}} \\
t^{p}=\frac{2 \cos \theta}{\cos \theta_{2}+\left(\frac{n_{2}}{n_{3}}\right) \cos \theta} \\
\cos \theta_{2}=\sqrt{1-\left(\frac{n_{3}}{n_{2}}\right)^{2} \operatorname{sen}^{2} \theta}, \quad k_{2}=k\left(\frac{\sin \alpha}{\sin \alpha_{2}}\right)
\end{array}
$$


Logo, essas modificações alteram o valor das integrais e dos coeficientes ea, e sendo se os indice de refreçao $n_{3}, n_{2}$ são iguas, temos, novamente, o caso das das Equaçoes 2.98 e 2.99 sem deslocamento focal. Essas equações também dão a forma da onda, logo, com esse novos fatores, essa forma é modificada, ou seja, o deslocamento focal altera a forma da onda incidente na amostra.

\subsection{Força eletromagnética em uma esfera}

Para determinar a força exercida pela pressão de radiação numa esfera dielétrica, com o comprimento de onda do laser da mesma ordem do diâmetro $d$ da esfera, em um meio não absorvedor e uma esfera não absorvedora, sendo essa condição uma aproximação, temos que a força óptica é dada pelo tensor de stress de Maxwell ${ }^{[4]}[1]$.

$$
F_{i}=\oint T_{i j} n_{j} d A=\frac{1}{2} \operatorname{Re} \oint\left[\varepsilon E_{i} E_{j}+\mu H_{i} H_{j}-\frac{1}{2}\left(\varepsilon E_{i} \cdot E_{j}+\mu H_{i} \cdot H_{j}\right) \delta_{i j} n_{j} d A\right]
$$

onde, usadas as aproximações assintópticas para funções de Bessel esférica e de Henkel, e integrando o tensor de estresse numa esfera com o limite de $k r \rightarrow \infty$, desprezamos os termos da equação de Henkel e Bessel para $\frac{1}{r^{s}}$ onde $s>2$. Sendo assim, considerando as duas soluções, montamos um tensor $v \sim{ }_{n}(k r)$, onde:

$$
v_{n}(k r)=\left[\begin{array}{c}
\frac{(-i)^{n+1} e^{i k r}}{2 k r}+\frac{(i)^{n+1} e^{-i k r}}{2 k r} \\
\frac{(-i)^{n+1} e^{i k r}}{k r}
\end{array}\right]
$$

Onde os temos de $v_{n}(k r)$ e $v_{n}^{\prime}(k r)$ permanecem e apenas descartamos os termos $v_{n}(k r) / k r$, portanto

$$
\begin{aligned}
& \nabla \times\left(v_{n}(k r) \vec{X}_{n m}(\theta, \phi)\right)=\nabla v_{n}(k r) \times \vec{X}_{n m}(\theta, \phi)+v_{n}(k r) \nabla \times \vec{X}_{n m}(\theta, \phi)= \\
& k v_{n}^{\prime}(k r)\left[\hat{r} \times \vec{X}_{n m}(\theta, \phi)\right]+v_{n}(k r) \nabla \times \vec{X}_{n m}(\theta, \phi) \cong k v_{n}^{\prime}(k r)\left[\hat{r} \times \vec{X}_{n m}(\theta, \phi)\right]
\end{aligned}
$$

Se desprezarmos o segundo termo, por se encaixar na condição de $v_{n}(k r) / k r$, ficaremos então com:

$$
\nabla \times\left(v_{n}(k r) \vec{X}_{n m}(\theta, \phi)\right)=\left[\begin{array}{c}
i k\left[\frac{(-i)^{n+1} e^{i k r}}{2 k r}-\frac{(i)^{n+1} e^{-i k r}}{2 k r}\right] \\
i k \frac{(-i)^{n+1} e^{i k r}}{k r}
\end{array}\right] \hat{r} \times \vec{X}_{n m}(\theta, \phi)
$$

Dessa forma, o campo total elétrico segundo o tensor, em relação aos campos incidentes e espalhados, ficará: 
$E_{0} \sum_{n, m}\left[\frac{i}{k_{1}} G_{n m}^{T M} \nabla \times j_{n}\left(k_{1} r\right) \vec{X}_{n m}(\theta, \phi)+G_{n m}^{T E} j_{n}\left(k_{1} r\right) \vec{X}_{n m}(\theta, \phi)\right]-\left[\sum_{n, m}\left[\frac{i}{k_{1}} a_{n} G_{n m}^{T M} \nabla \times\right.\right.$ $\left.\left.h_{n}^{1}\left(k_{1} r\right) \vec{X}_{n m}(\theta, \phi)+b_{n} G_{n m}^{T E} h_{n}^{1}\left(k_{1} r\right) \vec{X}_{n m}(\theta, \phi)\right]\right]=E_{0} \frac{e^{i k r}}{2 k r} \sum_{n, m}(-i)^{n+1}[(1-$ $\left.\left.2 b_{n}\right) G_{n m}^{T E} \vec{X}_{n m}-\left(1-2 a_{n}\right) G_{n m}^{T M} \hat{r} \times \vec{X}_{n m}\right]+E_{0} \frac{e^{-i k r}}{2 k r} \sum_{n, m}(i)^{n+1}\left[G_{n m}^{T E} \vec{X}_{n m}+G_{n m}^{T M} \hat{r} \times\right.$ $\left.\vec{X}_{n m}\right]$

A contribuição do campo elétrico para uma esfera é $k r \rightarrow \infty$, denominando $k_{1}$ de $k$ para facilitar a escrita da equação, logo, fazendo o produto vetorial com o seu conjugada onde temos os novos coeficientes $a_{p}, b^{*}{ }_{p}, G_{p q}^{T E *}, G_{p q}^{T M *}$, com os índice $p$ e $q$ que se relacionam a $n \mathrm{e}$ $m$ nas equações final da força óptica.

$$
\begin{array}{r}
\varepsilon \vec{E} \cdot \vec{E}^{*}=-\frac{\varepsilon\left|E_{0}\right|^{2}}{2(k r)^{2}}\left\{\sum _ { n , p , q , m } ( i ) ^ { p - n } \left[\left(b_{n}+b_{p}^{*}-2 b_{n} b_{p}^{*}\right) G_{n m}^{T E} G_{p q}^{T E *}+\left(a_{n}+a_{p}^{*}-\right.\right.\right. \\
\left.\left.2 a_{n} a_{p}^{*}\right) G_{n m}^{T M} G_{p q}^{T M *}\right]\left(\vec{X}_{n, m} \cdot \vec{X}_{p q}^{*}\right)+\left[\left(a_{n}+b_{p}^{*}-2 a_{n} b_{p}^{*}\right) G_{n m}^{T M} G_{p q}^{T E *}-\left(b_{n}+a_{p}^{*}-\right.\right. \\
\left.\left.2 b_{n} a_{p}^{*}\right) G_{n m}^{T E} G_{p q}^{T M *}\right] \vec{X}_{n, m} \cdot\left(\hat{r} \times \vec{X}_{p q}^{*}\right)-2 \operatorname{Re}\left[e ^ { i 2 k r } \sum _ { n , p , q , m } ( - i ) ^ { n - p } \left[\left(b_{n} G_{n m}^{T E} G_{p q}^{T E *}-\right.\right.\right. \\
\left.\left.\left.a_{n} G_{n m}^{T M} G_{p q}^{T M *}\right) \vec{X}_{n, m} \vec{X}_{p q}^{*}-\left(b_{n} G_{n m}^{T E} G_{p q}^{T M *}-a_{n} G_{n m}^{T M} G_{p q}^{T E *}\right) \vec{X}_{n, m} \cdot\left(\hat{r} \times \vec{X}_{p q}^{*}\right)\right]\right\}
\end{array}
$$

Utilizando $\quad$ os $\quad$ vetores $\left(\hat{r} \times \vec{X}_{n m}(\theta, \phi)\right) \cdot\left(\hat{r} \times \vec{X}_{p, q}^{*}(\theta, \phi)\right)=\vec{X}_{n, m} \cdot \vec{X}_{p q}^{*} \quad$ e $\vec{X}_{p, q}^{*} \cdot\left(\hat{r} \times X_{n, m}\right)=-\vec{X}_{n, m} \cdot\left(\hat{r} \times \vec{X}_{p q}^{*}\right)$. Nos termos que contém apenas $a_{n}$ ou $b_{n}$ os mesmos foram subtraídos, pois correspondem ao termo do feixe incidente. Desta forma, repetindo para campo magnético, temos:

$$
\vec{H}=
$$

$$
\begin{gathered}
H_{0} \sum_{n, m}\left[\frac{-i}{k_{1}} G_{n m}^{T M} \nabla \times j_{n}\left(k_{1} r\right) \vec{X}_{n m}(\theta, \phi)+G_{n m}^{T E} j_{n}\left(k_{1} r\right) \vec{X}_{n m}(\theta, \phi)\right]\left[\sum _ { n , m } \left[\frac{i}{k_{1}} b_{n} G_{n m}^{T E} \nabla \times\right.\right. \\
\left.\left.h_{n}^{1}\left(k_{1} r\right) \vec{X}_{n m}(\theta, \phi)-a_{n} G_{n m}^{T E} h_{n}^{1}\left(k_{1} r\right) \vec{X}_{n m}(\theta, \phi)\right]\right]
\end{gathered}
$$

E, o produto escalar dos campos magnéticos é: 


$$
\begin{array}{r}
\varepsilon \vec{H} \cdot \overrightarrow{H^{*}}=-\frac{\varepsilon\left|E_{0}\right|^{2}}{2(k r)^{2}}\left\{\sum _ { n , m , p , q } ( - i ) ^ { n - p } \left[\left(a_{n}+a_{p}^{*}-2 a_{n} a_{p}^{*}\right) G_{n m}^{T M} G_{p q}^{T M *}+\left(b_{n}+b_{p}^{*}-\right.\right.\right. \\
\left.\left.2 b_{n} b_{p}^{*}\right) G_{n m}^{T E} G_{p q}^{T E *}\right]\left(\vec{X}_{n, m} \cdot \vec{X}_{p q}^{*}\right)+\left[\left(a_{n}+b_{p}^{*}-2 a_{n} b_{p}^{*}\right) G_{n m}^{T M} G_{p q}^{T E *}-\left(b_{n}+a_{p}^{*}-\right.\right. \\
\left.\left.2 b_{n} a_{p}^{*}\right) G_{n m}^{T E} G_{p q}^{T M *}\right] \vec{X}_{n, m} \cdot\left(\hat{r} \times \vec{X}_{p q}^{*}\right)-2 \operatorname{Re}\left[e ^ { i 2 k r } \sum _ { n , p , q , m } ( - i ) ^ { n + p } \left[\left(a_{n} G_{n m}^{T M} G_{p q}^{T M *}-\right.\right.\right. \\
\left.\left.\left.b_{n} G_{n m}^{T E} G_{p q}^{T E *}\right) \vec{X}_{n, m} \cdot \vec{X}_{p q}^{*}-\left(b_{n} G_{n m}^{T E} G_{p q}^{T M *}+a_{n} G_{n m}^{T M} G_{p q}^{T E *}\right) \vec{X}_{n, m} \cdot\left(\hat{r} \times \vec{X}_{p q}^{*}\right)\right]\right\}
\end{array}
$$

Somando os dois campos e depois integrado de acordo com o tensor de Maxwell, obtemos a seguinte expressão:

$$
\begin{aligned}
& F_{i}=\frac{-\varepsilon\left|E_{0}\right|^{2}}{4 k^{2}}[R E] \sum_{n, m, p, q}(-i)^{n-p}\left[\left(a_{n}+a_{p}^{*}-2 a_{n} a_{p}^{*}\right) G_{n m}^{T M} G_{p q}^{T M *}\right. \\
&\left.+\left(b_{n}+b_{p}^{*}-2 b_{n} b_{p}^{*}\right) G_{n m}^{T E} G_{p q}^{T E *}\right] \int\left[\begin{array}{c}
\operatorname{sen} \theta \cos \phi \\
\operatorname{sen} \theta \operatorname{sen} \phi \\
\cos \phi
\end{array}\right] \vec{X}_{n, m} \cdot \vec{X}_{p q}^{*} d \Omega \\
&+\sum_{n, m, p, q}(-i)^{n-p}\left[\left(a_{n}+b_{n}^{*}-2 a_{n} b_{p}^{*}\right) G_{n m}^{T M} G_{p q}^{T E *}\right. \\
&\left.\left.-\left(b_{n}+a_{p}^{*}-2 b_{n} a_{p}^{*}\right) G_{n m}^{T E} G_{p q}^{T M *}\right)\right] \int\left[\begin{array}{c}
\operatorname{sen} \theta \cos \phi \\
\operatorname{sen} \theta \operatorname{sen} \phi \\
\cos \phi
\end{array}\right] \vec{X}_{n, m} \cdot\left(\hat{r} \times \vec{X}_{p q}^{*}\right) e^{i \phi} d \Omega
\end{aligned}
$$

E, utilizando o fato que

$$
\begin{gathered}
\sum_{n, m, p, q}(-i)^{n-p}\left[\left(a_{n}+a_{p}^{*}-2 a_{n} a_{p}^{*}\right) G_{n m}^{T M} G_{p q}^{T M *}\right. \\
\left.+\left(b_{n}+b_{p}^{*}-2 b_{n} b_{p}^{*}\right) G_{n m}^{T E} G_{p q}^{T E *}\right] \int \vec{X}_{n, m} \cdot \vec{X}_{p q}^{*} d \Omega= \\
\sum_{n, m, p, q}(-i)^{p-n}\left[\left(a_{p}+a_{n}^{*}-2 a_{p} a_{n}^{*}\right) G_{p q}^{T M} G_{n m}^{T M *}\right. \\
\left.+\left(b_{p}+b_{n}^{*}-2 b_{p} b_{n}^{*}\right) G_{p q}^{T E} G_{n m}^{T E *}\right] \int \vec{X}_{p, q} \cdot \vec{X}_{n m}^{*} d \Omega= \\
\sum_{n, m, p, q}(-i)^{n-p}\left[( a _ { n } + a _ { p } ^ { * } - 2 a _ { n } a _ { p } ^ { * } ) G _ { n m } ^ { T M } G _ { p q } ^ { T M * } \left(b_{n}+b_{p}^{*}-\right.\right. \\
\left.\left.\left.2 b_{n} b_{p}^{*}\right) G_{n m}^{T E} G_{p q}^{T E *}\right] \int \vec{X}_{n, m} \cdot \vec{X}_{p q}^{*} d \Omega\right\}^{*}
\end{gathered}
$$

E, portanto 


$$
\begin{gathered}
\sum_{n, m, p, q}(-i)^{n-p}\left[\left(a_{n}+a_{p}^{*}-2 a_{n} a_{p}^{*}\right) G_{n m}^{T M} G_{p q}^{T M *}+\left(b_{n}+b_{p}^{*}-2 b_{n} b_{p}^{*}\right) G_{n m}^{T E} G_{p q}^{T E *}\right] \int \vec{X}_{n, m} \\
\vec{X}_{p q}^{*} d \Omega=\text { real }
\end{gathered}
$$

No caso em que $\mathrm{C}$ é um número real, então temos que:

$$
\left(\begin{array}{l}
R e \\
I m
\end{array}\right) C e^{i \phi}=\left(\begin{array}{l}
C \cos \phi \\
C \operatorname{sen} \phi
\end{array}\right)
$$

Logo,

$$
\begin{aligned}
& \left(\begin{array}{l}
F_{x} \\
F_{y}
\end{array}\right)=\frac{-\varepsilon\left|E_{0}\right|^{2}}{4 k^{2}}\left[\begin{array}{l}
R e \\
I m
\end{array}\right] \sum_{n, m, p, q}(-i)^{n-p}\left[\left(a_{n}+a_{p}^{*}-2 a_{n} a_{p}^{*}\right) G_{n m}^{T M} G_{p q}^{T M *}+\left(b_{n}+b_{p}^{*}-\right.\right. \\
& \left.\left.2 b_{n} b_{p}^{*}\right) G_{n m}^{T E} G_{p q}^{T E *}\right] \int \operatorname{sen} \theta \vec{X}_{n, m} \cdot \vec{X}_{p q}^{*} e^{i \phi} d \Omega+ \\
& \sum_{n, m, p, q}(-i)^{n-p}\left[\left(a_{n}+b_{p}^{*}-2 a_{n} b_{p}^{*}\right) G_{n m}^{T M} G_{p q}^{T E *}-\right. \\
& \left.\left.\left(b_{n}+a_{p}^{*}-2 b_{n} a_{p}^{*}\right) G_{n m}^{T E} G_{p q}^{T M *}\right)\right] \int \operatorname{sen} \theta \vec{X}_{n, m}^{T} \cdot\left(\hat{r} \times \vec{X}_{p q}^{*}\right) e^{i \phi} d \Omega
\end{aligned}
$$

$$
\begin{array}{r}
F_{Z}=\frac{-\varepsilon\left|E_{0}\right|^{2}}{4 k^{2}} R e \sum_{n, m, p, q}(-i)^{n-p}\left[\left(a_{n}+a_{p}^{*}-2 a_{n} a_{p}^{*}\right) G_{n m}^{T M} G_{p q}^{T M *}+\left(b_{n}+b_{p}^{*}-\right.\right. \\
\left.\left.2 b_{n} b_{p}^{*}\right) G_{n m}^{T E} G_{p q}^{T E *}\right] \int \cos \theta \vec{X}_{n, m} \cdot \vec{X}_{p q}^{*} d \Omega+ \\
\sum_{n, m, p, q}(-i)^{n-p}\left[\left(a_{n}+b_{n}^{*}-2 a_{n} b_{p}^{*}\right) G_{n m}^{T E} G_{p q}^{T E *}-\right. \\
\left.\left(b_{n}+a_{p}^{*}-2 b_{n} a_{p}^{*} G_{n m}^{T E} G_{p q}^{T E *}\right)\right] \int \cos \theta \vec{X}_{n, m} \cdot\left(\hat{r} x \vec{X}_{p q}^{*}\right) d \Omega(2.126)
\end{array}
$$

A fim de solucionar o problema para determinar a força e avaliar as integrais nas expressões, usaremos as relações numéricas abaixo para deixar a integral numa forma mais simples. A dedução dessas relações não será desenvolvida no presente trabalho podendo ser consultada na literatura, sendo o seu valor ${ }^{[1][13]}$ :

$$
\begin{aligned}
& I_{1}=\int \cos \theta \vec{X}_{n, m} \cdot \vec{X}_{p q}^{*} d \Omega= \\
& \delta_{m, q} \frac{\delta_{p+1, m}}{(p+1)} \sqrt{\frac{p(p+2)}{(2 p+1)(2 p+3)}(p+m+1)(p-m+1)}+ \\
& \delta_{m, q} \frac{\delta_{n+1, m}}{(n+1)} \sqrt{\frac{n(n+2)}{(2 n+1)(2 n+3)}(n+m+1)(n-m+1)} \\
& I_{2}=\int \cos \theta \vec{X}_{n, m} \cdot\left(\hat{r} \times \vec{X}_{p q}^{*}\right) d \Omega=\delta_{q, m} \delta_{n, p} i m \frac{1}{n(n+1)} \\
& I_{3}=\int \operatorname{sen} \theta \vec{X}_{n, m} \cdot \vec{X}_{p q}^{*} e^{i \phi} d \Omega=\delta_{\mathrm{q}, \mathrm{m}+1} \frac{\delta_{\mathrm{n}, \mathrm{p}+1}}{(\mathrm{p}+1)} \sqrt{\frac{\mathrm{p}(\mathrm{p}+2)}{(2 \mathrm{p}+1)(2 \mathrm{p}+3)}(\mathrm{p}-\mathrm{m}+1)(\mathrm{p}-\mathrm{m})}+ \\
& \delta_{\mathrm{q}, \mathrm{m}+1} \frac{\delta_{\mathrm{p}, \mathrm{n}+1}}{(\mathrm{n}+1)} \sqrt{\frac{\mathrm{n}(\mathrm{n}+2)}{(2 \mathrm{n}+1)(2 \mathrm{n}+3)}(\mathrm{n}+\mathrm{m}+2)(\mathrm{n}-\mathrm{m}+1)}
\end{aligned}
$$




$$
I_{4}=\int \operatorname{sen} \theta \vec{X}_{n, m} \cdot\left(\hat{r} \times \vec{X}_{p q}^{*}\right) e^{i \phi} d \Omega=\delta_{q, m+1} \delta_{n, p} \frac{i}{n(n+1)} \sqrt{(n-m)(n+m+1)}
$$

Substituído nas forças longitudinal e transversal, obtemos as expressões finais para força no regime de Lorenz - Mie.

$$
F_{Z}=
$$$$
\frac{-\varepsilon\left|E_{0}\right|^{2}}{4 k^{2}} \sum_{n=1} \frac{1}{n+1}\left\{\sqrt { \frac { \mathrm { n } ( \mathrm { n } + 2 ) } { ( 2 \mathrm { n } + 1 ) ( 2 \mathrm { n } + 3 ) } } \sum _ { m = - n } ^ { n } \sqrt { ( \mathrm { n } + \mathrm { m } + 1 ) ( \mathrm { n } - \mathrm { m } + 1 ) } \operatorname { R e } \left[i \left(a_{n}+a_{n+1}^{*}-\right.\right.\right.
$$$$
\left.\left.2 a_{n} a_{n+1}^{*}\right) G_{n m}^{T M} G_{n+1, m}^{T M *}\right]+
$$

$$
\begin{aligned}
\operatorname{Re}\left[i\left(b_{n}+b_{n+1}^{*}-2 b_{n} b_{n+1}^{*}\right) G_{n m}^{T E} G_{n+1 m}^{T E *}\right]+ & \frac{1}{n} \sum_{m=-n}^{n} m \operatorname{Re}\left[i \left(a_{n}+b_{n}^{*}-\right.\right. \\
& \left.\left.\left.2 a_{n} b_{n}^{*}\right) G_{n m}^{T E} G_{n m}^{T E *}\right]\right\}
\end{aligned}
$$

$$
\begin{aligned}
& \left(\begin{array}{l}
F_{x} \\
F_{y}
\end{array}\right)=\frac{-\varepsilon\left|E_{0}\right|^{2}}{4 k^{2}}\left[\begin{array}{l}
R e \\
I m
\end{array}\right] \sum_{n=1} \frac{1}{n+1}\left\{\sqrt { \frac { \mathrm { n } ( \mathrm { n } + 2 ) } { ( 2 \mathrm { n } + 1 ) ( 2 \mathrm { n } + 3 ) } } \sum _ { m = - n } ^ { n } \sqrt { ( \mathrm { n } + \mathrm { m } + 1 ) ( \mathrm { n } - \mathrm { m } + 1 ) } \left[\left(a_{n+1}+\right.\right.\right. \\
& \left.a_{n}^{*}-2 a_{n+1} a_{n}^{*}\right) G_{n+1,-(m+1)}^{T M} G_{n,-m}^{T M *}+\left(a_{n}+a_{n+1}^{*}-2 a_{n} a_{n+1}^{*}\right) G_{n m}^{T M} G_{n+1, m+1}^{T M *}+ \\
& \left.\left(b_{n+1}+b_{n}^{*}-2 b_{n+1} b_{n}^{*}\right) G_{n+1,-(m+1)}^{T E} G_{n-m}^{T E *}+\left(b_{n}+b_{n+1}^{*}-2 b_{n} b_{n+1}^{*}\right) G_{n m}^{T E} G_{n+1, m+1}^{T E *}\right]- \\
& \frac{1}{n} \sum_{m=-n}^{n} \sqrt{(\mathrm{n}+\mathrm{m}+1)(\mathrm{n}-\mathrm{m}+1)} \sqrt{(\mathrm{n}+\mathrm{m}+1)(\mathrm{n}-\mathrm{m}+1)}\left[\left(a_{n}+b_{n}^{*}-\right.\right. \\
& \left.2 a_{n} b_{p}^{*}\right) G_{n m}^{T M} G_{n, m+1}^{T E *}-\left(b_{n}+a_{n}^{*}-2 b_{n} a_{n}^{*} G_{n m}^{T E} G_{n, m+1}^{T M *}\right)
\end{aligned}
$$

Logo, para determinar o coeficiente de choque usamos a equação

$$
c_{i}=c F_{i}
$$

Desta maneira, as equações ficam:

$$
\begin{gathered}
c_{z}=\frac{1}{2 k^{2}} \sum_{n=1} \frac{1}{n+1}\left\{\sqrt { \frac { \mathrm { n } ( \mathrm { n } + 2 ) } { ( 2 \mathrm { n } + 1 ) ( 2 \mathrm { n } + 3 ) } } \sum _ { m = - n } ^ { n } \sqrt { ( \mathrm { n } + \mathrm { m } + 1 ) ( \mathrm { n } - \mathrm { m } + 1 ) } \operatorname { R e } \left[i \left(a_{n}+\right.\right.\right. \\
\left.\left.a_{n+1}^{*}-2 a_{n} a_{p}^{*}\right) G_{n m}^{T M} G_{n+1, m}^{T M *}\right]+\operatorname{Re}\left[i\left(b_{n}+b_{n+1}^{*}-2 b_{n} b_{n+1}^{*}\right) G_{n m}^{T E} G_{n+1 m}^{T E *}\right]+ \\
\left.\frac{1}{n} \sum_{m=-n}^{n} m \operatorname{Re}\left[i\left(a_{n}+b_{n}^{*}-2 a_{n} b_{n}^{*}\right) G_{n m}^{T E} G_{n m}^{T E *}\right]\right\} \\
\left(\begin{array}{c}
c_{x} \\
c_{y}
\end{array}\right)=\frac{1}{4 k^{2}}\left[\begin{array}{c}
R e \\
I m
\end{array}\right] \sum_{n=1} \frac{1}{n+1}\left\{\sqrt { \frac { \mathrm { n } ( \mathrm { n } + 2 ) } { ( 2 \mathrm { n } + 1 ) ( 2 \mathrm { n } + 3 ) } } \sum _ { m = - n } ^ { n } \sqrt { ( \mathrm { n } + \mathrm { m } + 1 ) ( \mathrm { n } - \mathrm { m } + 1 ) } \left[\left(a_{n+1}+\right.\right.\right. \\
\left.a_{n}^{*}-2 a_{n+1} a_{n}^{*}\right) G_{n+1,-(m+1)}^{T M} G_{n,-m}^{T M *}+\left(a_{n}+a_{n+1}^{*}-2 a_{n} a_{n+1}^{*}\right) G_{n m}^{T M} G_{n+1, m+1}^{T M *}+ \\
\left.\left(b_{n+1}+b_{n}^{*}-2 b_{n+1} b_{n}^{*}\right) G_{n+1,-(m+1)}^{T E} G_{n-m}^{T E *}+\left(b_{n}+b_{n+1}^{*}-2 b_{n} b_{n+1}^{*}\right) G_{n m}^{T E} G_{n+1, m+1}^{T E *}\right]- \\
\frac{1}{n} \sum_{m=-n}^{n} \sqrt{(\mathrm{n}+\mathrm{m}+1)(\mathrm{n}-\mathrm{m}+1)} \sqrt{(\mathrm{n}+\mathrm{m}+1)(\mathrm{n}-\mathrm{m}+1)}\left[\left(a_{n}+b_{n}^{*}-\right.\right. \\
\left.\left.2 a_{n} b_{p}^{*}\right) G_{n m}^{T M} G_{n, m+1}^{T E *}-\left(b_{n}+a_{n}^{*}-2 b_{n} a_{n}^{*}\right) G_{n m}^{T E} G_{n, m+1}^{T M *}\right\}(2.135)
\end{gathered}
$$


Os coeficientes de choque do feixe foram colocados nessa forma, pois se torna mais fácil para simulá-los computacionalmente, como será demonstrado no próximo capítulo. 


\section{Referências}

[1] A. A. R. Neves. Força óptica em pinças ópticas: estudo teórico e experimental. 2006. 169 p. Tese (doutorado) - Universidade Estadual de Campinas, Instituto de Física Gleb Wataghin, Campinas, SP.

[2] K. Neuman, and S. Block, "Optical trapping,"The Review of Scientific Instruments, vol. 75, no. 9, pp. 2787-809, 2004.

[3] K. Svoboda, and S. Block, "Biological Applications of Optical Forces,"Annu Rev BiophBiom, vol. 23, no. 1, pp. 247-285, 1994.

[4] J. D. Jackson, Classical electrodynamics. Wiley, 2 ed., 1999.

[5] K. Visscher, and G. J. Brankenhoff, "Theoretical study of optically induced forces on spherical particles in a single beam trap.I: Rayleight scatterers," Optik, vol. 89, pp. 174-180, 1992.

[6] B. Richards, and E. Wolf, "Electromagnetic Diffraction in Optical Systems. II. Structure of the Image Field in an Aplanatic System,"Proceedings of the Royal Society A: Mathematical, Physical and Engineering Sciences, vol. 253, no. 1274, pp. 358-379, 1959.

[7]H. T. M. Van Der Voort, and G. J.Brakenhoff, "3-D image formation in high-aperture fluorescence confocal microscopy: A numerical analysis," J Microsc, vol. 158, no. 1, pp. 4354, 1990.

[8] S. M. Mansfild, and G.Kino,"Solid imersion microscope,"Appl Phys Lett, vol. 57, pp. 2615-2616, 1990.

[9] P. S. Alves. Teoria de calibração de pinça óptica. 2012. 74 p. Dissertação (mestrado) Universidade Federal de Viçosa, Viçosa, MG.

[10] A. Ashkin, "Forces of a single-beam gradient laser trap on a dielectric sphere in the ray optics regime," Biophysical Journal, vol. 61, no. 2, pp. 569-582, 1992.

[11] H. Kogelnik, and T. Li, "Laser beams and resonators," Appl. Opt., vol. 54, no. 10, pp. $1550-1567,1966$.

[12] M. Kerker, The scattering of light and other electromagnetic radiations. New York: Academia Press, 1969.

[13] G. Gouesbet, B. Maheu, and G. Grehan, "Light scattering from a sphere arbitrarily locatedin a Gaussian beam, using Bromwich formulation,"J Opt Soc Am A, vol. 5, no. 9, pp. 1427-1443,1988.

[14] G. B. Arkefen, H. J. Weber, and F. E. Harris, Mathematical Methods for Physicists: a comprehensive guide. Elsevier, 7 ed., 2013.

[15] K. F. Riley, M. P. Hobson and S. J. Bence, Mathematical methods for physics and engineering: a comprehensive guide. Cambridge: Cambridge University Press, 3 ed., 2019.

[16] P. A. M. Neto, and H. M Nussenzveig, “Theory of Optical Tweezer," Europhys Let, vol. 50, no. 5, pp. 702-708, 1999. 
[17] M. S. Rocha. Pinça óptica: experimento e teoria. 2004. 98 p.Dissertação (mestrado) Universidade Federal de Minas Gerais, Belo Horizonte, MG.

[18] N. B. Viena. Pinça óptica e aplicações. 2002. Tese (doutorado) - Universidade Federal de Minas Gerais, Belo Horizonte, MG.

[19] L. Novotny, and B. Hecht, Principles of Nano-Optics.Cambridge University Press, 2006.

[20] T. D. Visser, and S. H.Wiersma, "Diffraction of converging electromagnetic waves," $J$ Opt Soc Am A, vol. 9, no. 11, pp. 2034-2047, 1992.

[21] A. A. R. Neves, A. Fontes, C. L. Cesar, A. Camposeo, R. Cingolani, and D. Pisignano, "Axial optical trapping efficiency through a dielectric interface,"Physical Review E Statistical, Nonlinear, and Soft Matter Physics", vol. 76, no. 6, pp. 1-8, 2007.

[22] B. E. A. Saleh, and M. C. Teich, Fundamentals of photonics.Wiley, 2ed., 2006. 


\section{Capítulo3}

\section{Programa para calcular a força óptica}

\subsection{Introdução ao programa}

Nesse capítulo, abordaremos o programa usado para simular a pinça óptica, sendo ele feito a partir do modelo usado por NEVES $(2006)^{[1]}$, tendo o autor fornecido sua ajuda para desenvolver o programa aqui descrito. Este programa está apresentando nos Apêndices C, D E, além de estar dividido em 4 partes: cálculo da força axial com ou sem deslocamento focal, e cálculo da força radial com ou sem deslocamento focal. Esse programa foi feito na plataforma computacional de cálculo analítico "Mathematica", versão 11.3. Nesse capítulo, serão discutidos os processos matemáticos e computacionais para obtenção das equações simuladas no Apêndice, sendo que, essas transformações matemáticas se dão devido ao problema computacional para simular as expressões. Além disso, descreveremos o programa obtido através da derivação das integrais 2.106 a 2.109 que são usadas para calcular a constante $k$, sendo que esse programa foi desenvolvido a partir do programa apresentado no Apêndice, que, até onde sabemos, não estão presentes na literatura as simulações feitas da constante $k$.

\subsection{Simulação da força axial sem deslocamento focal}

O primeiro programa da força axial sem deslocamento focal está descrito no Apêndice $\mathrm{C}$, onde, considerando uma força só na direção $z_{0}$, e para $x_{0}=y_{0}=0$, e lembrando-se da definição das Equações 2.110 e 2.111 temos: $\rho_{0}=\sqrt{x_{0}^{2}+y_{0}^{2}}$ e $\phi_{0}=\tan ^{-1}\left[\frac{y_{0}}{x_{0}}\right]$, desta forma, as equações de Bessel somem, e, $m= \pm 1$ e $n$ vai de 0 à infinito, porém, como não podemos calcular até infinito, usamos uma relação de truncamento para determinar esse número, o detalhamento dessa expressão e sua explicação podem ser consultadas no Apêndice C. Assim, no limite, temos que as equações de Bessel ficam com a forma de:

$$
\lim _{k \rho_{0} \rightarrow 0} \frac{J_{m}\left(k p_{0} \operatorname{sen} \alpha^{\prime \prime}\right)}{k p_{0} \operatorname{sen} \alpha^{\prime \prime \prime}}=\frac{\left(k p_{0} \operatorname{sen} \alpha^{\prime \prime}\right)^{m-1}}{2^{n} m !}=\left\{\begin{array}{c} 
\pm\left(\frac{1}{2}\right) \text { para } m= \pm 1 \\
0 \text { para }|m| \neq 1
\end{array}\right.
$$




$$
\lim _{k \rho_{0} \rightarrow 0} \frac{J_{m}^{\prime}\left(k p_{0} \operatorname{sen} \alpha^{\prime \prime \prime}\right)}{1}=\frac{m\left(k p_{0} \operatorname{sen} \alpha^{\prime \prime \prime}\right)^{m-1}}{2^{n} m !}=\left\{\begin{array}{c} 
\pm\left(\frac{1}{2}\right) \text { para } m= \pm 1 \\
0 \text { para }|m| \neq 1
\end{array}\right.
$$

Essa relação não será demonstrada, porém, pode ser consultada nas referências [3] e [4]. Logo, aplicado essa condição e a mudança de variável $\cos \alpha^{\prime \prime \prime}=x$, nas Equações 2.112 a 2.115, chegamos a uma única integral. E, utilizando a relação $\operatorname{sen}^{2} \alpha^{\prime \prime \prime}\left(P_{n}^{\prime m}\left(\cos \left(\alpha^{\prime \prime \prime}\right)\right)=\right.$ $\left.m \cos \left(\alpha^{\prime \prime \prime}\right)\right) P_{n}^{m}\left(\cos \left(\alpha^{\prime \prime \prime}\right)\right)+(m+n)(-m+n+1) \operatorname{sen} \alpha P_{n}^{m-1}\left(\cos \left(\alpha^{\prime \prime \prime}\right)\right)$, chegamos à integral:

$$
I(n)=\int_{\theta_{\max }}^{1} d \alpha \sqrt{x} e^{-\frac{f^{2}\left(1-x^{2}\right)}{\omega_{0}^{2}}} e^{-i z_{0} x}\left(P_{n}^{m}(x)-\sqrt{1-x^{2}}(n+1) n P_{n}(x)\right)
$$

Que é calculada no programa e está apresentada no Apêndice C.

Agora, aplicado as mesmas condições da integral na equação da forma do campo 2.110 e 2.111, chegamos nas relações da forma do campo incidente quando está apenas na direção axial.

$$
\begin{aligned}
& G_{n \pm 1}^{T M}= \pm i 4 \pi i^{n-m} \frac{i k f e^{-i k f}}{n(n+1)} \sqrt{\frac{n_{0}}{n_{3}}} \sqrt{\frac{2 n+1}{4 \pi} I(n)} \\
& G_{n \pm 1}^{T E}=4 \pi i^{n-m} \frac{i k f e^{-i k f}}{n(n+1)} \sqrt{\frac{n_{0}}{n_{3}}} \sqrt{\frac{2 n+1}{4 \pi}} I(n)
\end{aligned}
$$

Vendo que eles são ortogonais, temos que $G_{n \pm 1}^{T M} G_{n \pm 1}^{T E *}=0$, logo, aplicado essas condições na Equação 2.131, retirado as partes que não depende de n, chegamos na equação:

$$
c_{z}=\sum_{n=1} \frac{1}{(n+1)} \operatorname{Re}\left[i\left(a_{n}+a_{n+1}^{*}-2 a_{n} a_{p}^{*}\right) I(n) I^{*}(n)\right]+\operatorname{Re}\left[i \left(b_{n}+b_{n+1}^{*}-\right.\right.
$$

$\left.\left.2 b_{n} b_{n+1}^{*}\right) I(n) I^{*}(n)\right]$

onde, aplicando as constantes, chegamos à equação para força resultante. Com $\varepsilon E^{2}{ }_{0}=$ $P / c \pi w^{2}{ }_{0} n_{2}{ }^{[2]}$, sendo $P$ a potência do laser em seu centro.

$$
\begin{gathered}
F_{z}=\pi(f * k)^{2}\left(\frac{1}{2}\right) \sqrt{\frac{n_{0}}{n_{3}}} \varepsilon E_{0}^{2} \sum_{n=1} \frac{1}{(n+1)} \operatorname{Re}\left[i\left(a_{n}+a_{n+1}^{*}-2 a_{n} a_{p}^{*}\right) I(n) I^{*}(n)\right]+\operatorname{Re}\left[i \left(b_{n}+\right.\right. \\
\left.\left.b_{n+1}^{*}-2 b_{n} b_{n+1}^{*}\right) I(n) I^{*}(n)\right]
\end{gathered}
$$

O resultado dessa simulação será apresentado no Capítulo 4 juntamente com os dados experimentais. 


\subsection{Simulação da força axial com deslocamento focal}

Essa simulação completa está descrita no Apêndice C.

Se considerarmos as Equações 2.120 e 2.121 e aplicarmos as mesmas condições das Equações 3.1 a 3.3, temos as novas integrais.

$I^{\prime}(n)=$

$\int_{\theta_{\max }}^{1} \sqrt{x}\left(P_{n}^{1}(z(x))(t s(x)-\operatorname{tp}(x) z(x))-\right.$

$\left.n(n+1)\left(\frac{n 3}{n 2}\right) \sqrt{1-x^{2}} t p(x) P_{n}(z(x))\right) \exp \left(f \omega^{2}\left(-\left(1-x^{2}\right)\right)-i(k l x+k 2(x o-l) z(x))\right.$

Onde $x_{2}=\cos \left(\alpha_{2}\right)=\sqrt{1-\left(\frac{n_{3}}{n_{2}}\right)^{2}\left(1-x^{2}\right)}$ da Equação 2.124, e $t^{p}, t^{s}$ são as equações 2.122 e 2.123 e $k_{2}$ e o mesmo da Equação 2.124 .

Então, essa integral será única, pois as condições 3.1 e 3.2 são satisfeitas. Logo, aplicamos essa nova integral à Equação 2.134 e chegamos a uma Equação análoga à 3.6.

$$
\begin{gathered}
{c^{\prime}}_{z}=\sum_{n=1} \frac{1}{(n+1)^{2}} \sqrt{\frac{n}{n+1}} \operatorname{Re}\left[i\left(a_{n}+a_{n+1}^{*}-2 a_{n} a_{p}^{*}\right) I(n) I^{*}(n)\right]+\operatorname{Re}\left[i \left(b_{n}+b_{n+1}^{*}-\right.\right. \\
\left.2 b_{n} b_{n+1}^{*}\right) I^{\prime}(n) I^{\prime *}(n)
\end{gathered}
$$

Sendo que, as equações de forma da onda 3.4 e 3.5 assumem a forma:

$$
\begin{aligned}
& G_{n \pm 1}^{T M}= \pm i 4 \pi i^{n-m} \frac{i k f e^{-i k f}}{n(n+1)} \sqrt{\frac{n_{0} n_{2}}{n_{3}}} \sqrt{\frac{2 n+1}{4 \pi}} I^{\prime}(n), \\
& G_{n \pm 1}^{T E}=4 \pi i^{n-m} \frac{i k f e^{-i k f}}{n(n+1)} \sqrt{\frac{n_{0} n_{2}}{n_{3}}} \sqrt{\frac{2 n+1}{4 \pi}} I^{\prime}(n) .
\end{aligned}
$$

E, a força fica na forma de:

$$
\begin{gathered}
F_{z}^{\prime}=\left(\frac{1}{2}\right) \pi(f * k)^{2} \sqrt{\frac{n_{0} n_{2}}{n_{3}}} \varepsilon E^{2}{ }_{0} \sum_{n=1} \frac{1}{(n+1)} \operatorname{Re}\left[i\left(a_{n}+a_{n+1}^{*}-2 a_{n} a_{p}^{*}\right) I(n) I^{*}(n)\right]+ \\
\operatorname{Re}\left[i\left(b_{n}+b_{n+1}^{*}-2 b_{n} b_{n+1}^{*}\right) I^{\prime}(n) I^{\prime *}(n)\right.
\end{gathered}
$$

Essa força também foi calculada e seu gráfico será apresentado no Capítulo 5.

\subsection{Cálculos da constante elástica $k$ a partir da força simulada axial}

Como não tínhamos o equipamento para calibragem da pinça no eixo $z$, o cálculo da constante elástica $k$ não pode ser comparado, mas o cálculo foi feito mesmo assim. Seria necessário derivar a força final 3.7 e 3.12, no entanto, no programa, a componente $z_{0}$ é definida como variável discreta e não contínua, uma vez que a mesma se torna 
computacionalmente grande se a tratarmos como contínua. Assim sendo, as Equações 3.6 e 3.9 foram derivadas em relação à $z_{0}$, onde, o que depende dessa variável são as integrais 3.3 e 3.8 que são derivadas em relação a $z_{0}=0$, assim:

$$
\frac{\partial I\left(z_{0}=0\right)}{\partial z_{0}}=\int_{\theta_{\max }}^{1} d x\left(i k x^{\frac{3}{2}} e^{-f \omega^{2}\left(1-x^{2}\right)}\left((1-x) P_{n}^{1}(x)-n(n+1) \sqrt{1-x^{2}} P_{n}(x)\right)\right.
$$

e

$$
\begin{array}{r}
\frac{\partial I_{z}^{\prime}\left(z_{0}=0\right)}{\partial z_{0}}= \\
\int_{\theta_{\text {max }}}^{1} d x i \mathrm{k} 2 \sqrt{x} z(x) \exp \left(\mathrm{f} \omega^{2}\left(-\left(1-x^{2}\right)\right)-i(k l x-\mathrm{k} 2 \lg (x))\right)\left(P_{n}^{1}(z(x))(\operatorname{ts}(x)-\right. \\
\left.\operatorname{tp}(x) z(x))-n(n+1)\left(\frac{\mathrm{n} 3}{\mathrm{n} 2}\right) \sqrt{1-x^{2}} \operatorname{tp}(x) P_{n}(z(x))\right)
\end{array}
$$

Os valores para diferentes potências estão apresentados no Capítulo 5.

\subsection{Simulações da força radial sem deslocamento focal}

O programa usado é semelhante ao da força axial e consta no Apêndice D. Agora, as integrais são submetidas às condições de $z_{0}=0$, além do que veremos no caso experimental, onde a força varia em relação aos eixos. Primeiro, variamos $x_{0}>0 \mathrm{e}$, depois, variamos $x_{0}<0$. O mesmo foi feito para $y_{0}$. Aplicado essas condições nas Equações 2.110 a 2.115, com a relação a equação $\tan ^{-1} \frac{y_{0}}{x_{0}}=\phi_{0}$, temos os valores assumidos por $\phi_{0}$ são $\phi_{0}=$ $0, \pi$ ou $\pm \pi / 2$, assim, as integrais são separadas em duas:

$$
\begin{gathered}
\int_{0}^{\theta_{\max }} d \alpha \sqrt{\cos \alpha^{\prime \prime \prime}} e^{-\frac{f^{2} \operatorname{sen}^{2} \alpha^{\prime \prime \prime}}{\omega_{0}^{2}}}\left\{m^{2} P_{n}^{m}\left(\cos \alpha^{\prime \prime \prime}\right) \frac{J_{m}\left(k p_{0} \operatorname{sen} \alpha^{\prime \prime \prime}\right)}{k p_{0} \operatorname{sen} \alpha^{\prime \prime}}-\right. \\
\left.\operatorname{sen}^{2} \alpha P_{n}^{\prime m}\left(\cos \alpha^{\prime \prime \prime}\right) J_{m}^{\prime}\left(k \rho_{0} \operatorname{sen} \alpha^{\prime \prime \prime}\right)\right\} \\
\int_{0}^{\theta_{\max }} d \alpha \sqrt{\cos \alpha^{\prime \prime \prime}} e^{-\frac{f^{2} \operatorname{sen}^{2} \alpha^{\prime \prime \prime}}{\omega_{0}^{2}}}\left\{i m P_{n}^{m}\left(\cos \alpha^{\prime \prime \prime}\right) J_{m}^{\prime}\left(k \rho_{0} \operatorname{sen} \alpha^{\prime \prime \prime}\right)-\right. \\
\left.\sin ^{2} \alpha^{\prime \prime \prime}\left(\cos \alpha^{\prime \prime \prime}\right) \frac{J_{m}\left(k p_{0} \operatorname{sen} \alpha^{\prime \prime}\right)}{k p_{0} \operatorname{sen} \alpha^{\prime \prime \prime}}\right\}
\end{gathered}
$$

Onde, a parte da exponencial com $z_{0}$ se anula. Logo, aplicando as mesmas mudanças de variáveis e aproximações usadas na parte axial, mais uma aproximação para a derivada da função de Bessel para o cálculo da integral que não depende do tempo, obtemos: 


$$
J_{m}^{\prime}\left(k p_{0} \operatorname{sen} \alpha^{\prime \prime \prime}\right)=m \frac{J_{m}\left(k p_{0} \operatorname{sen} \alpha^{\prime \prime \prime}\right)}{k p_{0} \operatorname{sen} \alpha^{\prime \prime \prime}}-j_{m+1}\left(k p_{0} \operatorname{sen} \alpha^{\prime \prime \prime}\right)
$$

Com isso, chegamos nas integrais:

$$
\begin{gathered}
I_{1}=\int_{\theta_{\text {max }}}^{1} d x \sqrt{x} e^{-\frac{f^{2}\left(1-x^{2}\right)}{\omega_{0}^{2}}}\left\{m P _ { n } ^ { m } ( x ) \left(\frac{x J_{m+1}\left(k p_{0} \sqrt{1-x^{2}}\right)}{\sqrt{1-x^{2}}} \frac{m J_{m}\left(k p_{0} \sqrt{1-x^{2}}\right)}{k p_{0}(x+1)}-(m+\right.\right. \\
\left.n)(-m+n+1) \operatorname{sen}^{2} \alpha P_{n}^{m-1}(x) \frac{m J_{m}\left(k p_{0} \sqrt{1-x^{2}}\right)}{k p_{0}(x+1)} J_{m+1}\left(k p_{0} \sqrt{1-x^{2}}\right)\right\} \\
I_{2}=\int_{\theta_{\max }}^{1} d x \sqrt{x} e^{-\frac{f^{2}\left(1-x^{2}\right)}{\omega_{0}^{2}}}\left\{P_{n}^{m}(x)\left(\frac{m J_{m}\left(k p_{0} \sqrt{1-x^{2}}\right)}{k p_{0}(x+1)}-\frac{J_{m+1}\left(k p_{0} \sqrt{1-x^{2}}\right)}{\sqrt{1-x^{2}}}\right)-(m+n)(-m+\right. \\
\left.n+1) \operatorname{sen}^{2} \alpha P_{n}^{m-1}(x) \frac{J_{m}\left(k p_{0} \sqrt{1-x^{2}}\right)}{k p_{0}(x+1)}\right\}
\end{gathered}
$$

Então, iremos calcular a nova forma dos coeficientes 2.110 e 2.111 que, sendo aplicadas as condições descritas nesse texto, tornam-se:

$$
\begin{gathered}
G_{n m}^{T M}=4 \pi i^{n-m} i k f e^{-i k f} X^{m+1} \sqrt{\frac{n_{0}}{n_{3}}} \sqrt{\frac{2 n+1(n-m) !}{4 \pi n(n+1)(n+m) !}} I_{1}\left(n, n, z_{0}=0\right) \\
G_{n m}^{T E}=i m 4 \pi i^{n-m} X^{m+1} i k f e^{-i k f} \sqrt{\frac{n_{0}}{n_{3}}} \sqrt{\frac{2 n+1(n-m) !}{4 \pi n(n+1)(n+m) !}} I_{2}\left(n, m, z_{0}=0\right) \\
X=\left\{\begin{array}{c}
1 x_{0}>0 \\
-1 x_{0}<0
\end{array}\right. \\
G_{n m}^{T M}=i m 4 \pi i^{n-m} i k f e^{-i k f} Y^{m+1} \sqrt{\frac{n_{0}}{n_{3}}} \sqrt{\frac{2 n+1(n-m) !}{4 \pi n(n+1)(n+m) !}} I_{2}\left(n, n, z_{0}=0\right) \\
G_{n m}^{T E}=-4 \pi i^{n-m} Y^{m+1} i k f e^{-i k f} \sqrt{\frac{n_{0}}{n_{3}}} \sqrt{\frac{2 n+1(n-m) !}{4 \pi n(n+1)(n+m) !}} I_{1}\left(n, m, z_{0}=0\right) \\
Y=\left\{\begin{array}{c}
i y_{0}>0 \\
-i y_{0}<0
\end{array}\right.
\end{gathered}
$$

Agora, para calcular a pressão de radiação, substituímos essas equações na Equação 2.135, e, com essa equação chegamos ao valor da força apresentado pela Equação 2.132, utilizando, para tal, o programa que consta no Apêndice C. 


\subsection{Simulações da força radial com deslocamento focal}

Da mesma forma que no movimento axial temos que, para o radial, considerando as Equações 2.1202 .121 e as condições da Secção 3.5, temos que a novas integrais para $\rho_{0}=0$ são:

$$
\begin{aligned}
& I_{1}^{\prime}=\int_{\theta_{\max }}^{1} \sqrt{x} \exp \left(f \omega^{2}\left(-\left(1-x^{2}\right)\right)-i k l x\right. \\
& +i k 2 l z(x))\left(\frac{m^{2}(t s(x)-t p(x) z(x)) J_{m}\left(k \sqrt{1-x^{2}} \rho_{0}\right) P_{n}^{m}(z(x))}{k\left(1-x^{2}\right) \rho_{0}}\right. \\
& +m \operatorname{tp}(x) z(x) J_{m+1}\left(k \sqrt{1-x^{2}} \rho_{0}\right) P_{n}^{m}(z(x))-\sqrt{1-x^{2}}(-m+n+1)(m \\
& \left.+n) \operatorname{tp}(x)\left(\frac{m J_{m}\left(k \sqrt{1-x^{2}} \rho_{0}\right)}{k \sqrt{1-x^{2} \rho_{0}}}-J_{m+1}\left(k \sqrt{1-x^{2} \rho_{0}}\right)\right) P_{n}^{m-1}(z(x))\right)
\end{aligned}
$$

$I_{2}^{\prime}=$

$\sqrt{x} \exp \left(\mathrm{f} \omega^{2}\left(-\left(1-x^{2}\right)\right)-i k l x+i \mathrm{k} 2 l z(x)\right)\left(-\frac{(-m+n+1)(m+n) \operatorname{tp}(x) J_{m}\left(k \sqrt{1-x^{2}} \rho_{0}\right) P_{n}^{m-1}(z(x))}{k \rho_{0}}-\right.$

$$
\begin{aligned}
& \quad \frac{m \operatorname{tp}(x) z(x) J_{m}\left(k \sqrt{1-x^{2}} \rho_{0}\right) P_{n}^{m}(z(x))}{k\left(1-x^{2}\right) \rho_{0}}+\frac{m \operatorname{ts}(x) J_{m}\left(k \sqrt{1-x^{2}} \rho_{0}\right) P_{n}^{m}(z(x))}{k \sqrt{1-x^{2} \rho_{0}}}- \\
& \left.\operatorname{ts}(x) J_{m+1}\left(k \sqrt{1-x^{2}} \rho_{0}\right) P_{n}^{m}(z(x))\right)
\end{aligned}
$$

Como as Equações 3.19 a 3.22 só depende de $m, n, k, f$, e dos índices de refração e das integrais, as equações dos coeficientes da forma da onda só terão mudança no índice de refração. Agora, considerando o índice de refração da água, as novas integrais resultam nas expressões:

$$
G_{n m}^{T M}=4 \pi i^{n-m} i k f e^{-i k f} X^{m+1} \sqrt{\frac{n_{0} n_{2}}{n_{3}}} \sqrt{\frac{2 n+1(n-m) !}{4 \pi n(n+1)(n+m) !}} I_{1}\left(n, m, t^{S}, t^{p}, z_{0}=0\right)
$$

$$
\begin{gathered}
G_{n m}^{T E}=i m 4 \pi i^{n-m} X^{m+1} i k f e^{-i k f} \sqrt{\frac{n_{0} n_{2}}{n_{3}}} \sqrt{\frac{2 n+1(n-m) !}{4 \pi n(n+1)(n+m) !}} I_{2}\left(n, m, t^{s}, t^{p}, z_{0}=0\right) \\
X=\left\{\begin{array}{cc}
1 & x_{0}>0 \\
-1 & x_{0}<0
\end{array}\right.
\end{gathered}
$$




$$
\begin{gathered}
G_{n m}^{T M}=i m 4 \pi i^{n-m} i k f e^{-i k f} Y^{m+1} \sqrt{\frac{n_{0} n_{2}}{n_{3}}} \sqrt{\frac{2 n+1(n-m) !}{4 \pi n(n+1)(n+m) !}} I_{2}\left(n, n, t^{S}, t^{p} z_{0}=0\right) \\
G_{n m}^{T E}=-4 \pi i^{n-m} Y^{m+1} i k f e^{-i k f} \sqrt{\frac{n_{0} n_{2}}{n_{3}}} \sqrt{\frac{2 n+1(n-m) !}{4 \pi n(n+1)(n+m) !}} I_{1}\left(n, m, t^{S}, t^{p} z_{0}=0\right) \\
Y=\left\{\begin{array}{cc}
i & y_{0}>0 \\
-i & y_{0}<0
\end{array}\right.
\end{gathered}
$$

Essas novas equações de força foram simuladas através do programa encontrado no Apêndice E, e, o resultado será apresentado no Capítulo 5.

\subsection{Cálculo da constante elástica $\mathbf{k}$ a partir da força simulada Radial}

Como feito para as forças axiais, as integrais foram derivadas em relação a $\rho_{0}$, obtendo:

$$
\begin{aligned}
& \frac{\partial I_{1}\left(\rho_{0} \approx 0\right)}{\partial \rho_{0}}= \\
& \sqrt{x} \exp \left(\mathrm{f} \omega^{2}\left(-\left(1-x^{2}\right)\right)-i k l x+i \mathrm{k} 2 l z(x)\right)\left(\frac{m \mathrm{n} 3(-m+n+1)(m+n) \operatorname{tp}(x) J_{m}\left(k \sqrt{1-x^{2}} \rho_{0}\right) P_{n}^{m-1}(z(x))}{k \mathrm{n} 2 \rho_{0}^{2}}-\right. \\
& \left.\frac{m^{2}(\operatorname{ts}(x)-\operatorname{tp}(x) z(x)) J_{m}\left(k \sqrt{1-x^{2}} \rho_{0}\right) P_{n}^{m}(z(x))}{k \rho_{0}^{2}\left(1-x^{2}\right)}\right)
\end{aligned}
$$

$\left.\frac{m \operatorname{tp}(x) z(x) J_{m}\left(k \sqrt{1-x^{2}} \rho_{0}\right) P_{n}^{m}(z(x))}{k \rho_{0}^{2}\left(1-x^{2}\right)}-\frac{m \operatorname{ts}(x) J_{m}\left(k \sqrt{1-x^{2}} \rho_{0}\right) P_{n}^{m}(z(x))}{k \rho_{0}^{2} \sqrt{1-x^{2}}}\right)$

Essas são as novas equações para caso sem deslocamento focal, onde aplicamos as Equações 3.19 a 3.22 e a substituímos na equação da força radial 2.132, isso nos dá o valor de $k$ radial. Esse valor, calculado para diferentes potências, será mostrado no Capítulo 5, onde usamos a mesma relação $3.16 \mathrm{e}$, desconsiderando e derivando em relação a $\rho_{0}$ no denominador. Logo, como $\rho_{0}$ é da ordem de mícrons, seu valor será $10^{6}$ maior em relação à parte da derivada de Bessel. Portanto, foi possível desprezar essa parte, o que resulta nessa expressão da derivada mostrada acima (equação 3.30). 
Para o caso das integrais derivadas em relação ao fenômeno não focal, das Equações 3.17 e 3.18 , essa equação deriva em relação a $\rho_{0}$, temos:

$$
\begin{array}{r}
\frac{\partial I_{1}^{\prime}\left(\rho_{0} \approx 0\right)}{\partial \rho_{0}} \\
\sqrt{x} e^{-\mathrm{f} \omega^{2}\left(1-x^{2}\right)}\left(\frac{m^{2} P_{n}^{m}(x) J_{m+1}\left(k \sqrt{1-x^{2}} \rho_{0}\right) x\left(J_{m-1}\left(k \sqrt{1-x^{2}} \rho_{0}\right)-J_{m+1}\left(k \sqrt{1-x^{2}} \rho_{0}\right)\right)}{2 \rho_{0}(x+1)}+\right. \\
\frac{m^{2} x P_{n}^{m}(x) J_{m}\left(k \sqrt{1-x^{2}} \rho_{0}\right)\left(J_{m}\left(k \sqrt{1-x^{2}} \rho_{0}\right)-J_{m+2}\left(k \sqrt{1-x^{2}} \rho_{0}\right)\right)}{2 \rho_{0}(x+1)} \\
\frac{\frac{m^{2} x P_{n}^{m}(x) J_{m}\left(k \sqrt{1-x^{2}} \rho_{0}\right) J_{m+1}\left(k \sqrt{1-x^{2}} \rho_{0}\right)}{\rho_{0}^{2} \sqrt{1-x^{2}} k(x+1)}}{(m+n) m(-m+n+1) P_{n}^{m-1}(x)\left(J_{m-1}\left(k \sqrt{1-x^{2}} \rho_{0}\right)-J_{m+1}\left(k \sqrt{1-x^{2}} \rho_{0}\right)\right) J_{m+1}\left(k \sqrt{1-x^{2}} \rho_{0}\right)} \\
\left.\frac{2 \rho_{0}}{2 \rho_{0}} \rho_{0}\right)\left(J_{m}\left(k \sqrt{1-x^{2}} \rho_{0}\right)-J_{m+2}\left(k \sqrt{1-x^{2}} \rho_{0}\right)\right) \\
\frac{(m+n) m(-m+n+1) P_{n}^{m-1}(x) J_{m}\left(k \sqrt{1-x^{2}}\right)}{k \rho_{0}^{2} \sqrt{1-x^{2}}}
\end{array}
$$

$$
\begin{array}{r}
\frac{\partial I^{\prime}{ }_{2}\left(\rho_{0} \approx 0\right)}{\partial \rho_{0}}= \\
\sqrt{x} e^{-\mathrm{f} \omega^{2}\left(1-x^{2}\right)}\left(-\frac{(-m+n+1)(m+n) P_{n}^{m-1}(x)\left(J_{m-1}\left(k \sqrt{1-x^{2}} \rho_{0}\right)-J_{m+1}\left(k \sqrt{1-x^{2}} \rho_{0}\right)\right)}{2 \rho_{0}}+\right. \\
\frac{(-m+n+1)(m+n) P_{n}^{m-1}(x) J_{m}\left(k \sqrt{1-x^{2}} \rho_{0}\right)}{k \rho_{0}^{2} \sqrt{1-x^{2}}}+P_{n}^{m}(x)\left(-\frac{m J_{m}\left(k \sqrt{1-x^{2}} \rho_{0}\right)}{\rho_{0}^{2} k(x+1)}-\frac{1}{2} k\left(J_{m}\left(k \sqrt{1-x^{2}} \rho_{0}\right)-\right.\right. \\
\left.\left.\left.J_{m+2}\left(k \sqrt{1-x^{2}} \rho_{0}\right)\right)+\frac{m \sqrt{1-x^{2}}\left(J_{m-1}\left(k \sqrt{1-x^{2}} \rho_{0}\right)-J_{m+1}\left(k \sqrt{1-x^{2}} \rho_{0}\right)\right)}{2 \rho_{0}(x+1)}\right)\right)
\end{array}
$$

Essas são equações usadas na Equação 2.132 e fornecem a constante $k$ com deslocamento focal. O valor dessa constante para diferentes valores de potência está demonstrado no apítulo 5. Onde, diferente do caso axial, as integrais divergem em $\rho_{0}=0$, sendo necessário calcular a função para pontos específicos, que serão explicados no Capítulo 5 . A equação da derivada de Bessel é diferente do caso com deslocamento focal, pois essa última gerou erro no programa não sendo possível resolver a tempo, logo, usei a relação matemática $j_{n}^{\prime}(x)=\frac{1}{2}\left(J_{n-1}(x)-\right.$ $\left.J_{n+1}(x)\right)$ nas Equações 3.17 e 3.18 para obter as Equações 3.31 e 3.32 . 


\subsection{O programa utilizado na análise dos dados do movimento Browniano}

Para análise do movimento browniano, utilizamos o programa desenvolvido por mim com auxílio do professor Vito Vanin e do meu orientador Adriano Alencar. Este programa está descrito no Apêndice F. Através dele, aumentamos o tamanho dos pixels brancos e delimitamos as bordas das partes em branco que foram aumentadas. Esse aumento se deve ao fato de o programa modificar os valores de intensidade de cor de uma parte próxima a máxima branca, o que na escala seria 255 em forma circular. Posteriormente, alteramos os valores das cores para seu extremo, ou seja, uma parte branca com valor de 255 é transformada em uma parte preta de valor 0 , e, em outra etapa, exportamos todos frames que compõem o vídeo para uma pasta onde eles estão em formato bmp. Depois, utilizamos um programa de renomeação de nome de arquivos gratuito chamado "Advance Reanemer" para deixar os frames com nome adequado para serem analisados no programa desenvolvido em $\mathrm{C}++$ pelo nosso laboratório. Este programa toma a posição em pixel das beads pretas bem definidas, desta forma, obtemos então, uma tabela da posição em pixel do centro das as microesferas e, a partir daí, é possível utilizá-las para calcular o valor de deslocamento médio do tempo do centro. 


\section{Referências}

[1]A. A. R. Neves. Força óptica em pinças ópticas: estudo teórico e experimental. 2006. 169 p. Tese (doutorado) - Universidade Estadual de Campinas, Instituto de Física GlebWataghin, Campinas, SP.

[2] Y. Harada, and T. Asakura, "Radiation forces on a dielectric sphere in the Rayleigh scattering regime,"Optics Communications, vol. 124, pp. 529-541, 1996.

[3] G. B. Arkefen, H. J. Weber, and F. E. Harris, Mathematical Methods for Physicists: a comprehensive guide. Elsevier, 7 ed., 2013.

[4]K. F. Riley, M. P. Hobson and S. J. Bence, Mathematical methods for physics and engineering: a comprehensive guide. Cambridge: Cambridge University Press, 3 ed., 2019. 


\section{Capítulo 4}

\section{Montagem experimental}

\subsection{O esquema da pinça}

A pinça óptica ${ }^{[1][5]}$, em sua forma mais simples, pode ser montada a partir de um microscópio invertido, sendo constituída de um laser de potência mínima de $10 \mathrm{~mW}$, um conjunto de componentes ópticos, um sensor de captura conectado ao computador e uma objetiva de grande abertura numérica e ampliação. Todos esses componentes devem estar sobre uma mesa óptica para evitar possíveis vibrações, além de ser necessário um motor piezelétrico ou de passo para movimentar a amostra e calibrar adequadamente a pinça. Fora de sua forma mais simples, a pinça óptica é feita com vários lasers para perturbar o objeto pinçado, além de também serem usados sistemas de calibração em $z$, e induzir modos de ressonância de Mie MDR. Algumas pinças ópticas já construídas por outros grupos estão acopladas a outras ferramentas, como, por exemplo, equipamentos de espectroscopia de florescência e espectofotômetro de luz. Nosso grupo pretende associar a construção de nossa pinça ao equipamento de Microscopia de Força e Tarção ou Tracion Force Microscopy (TFM), no futuro.

Decorrente de fatores únicos na montagem e calibração da pinça, sua montagem e manuseio se tornam difíceis para profissionais quem não são da área. Este fator proporcionou o aparecimento de pinça ópticas comerciais, como a da Thorlabs, Lumicks, entre outras, pois as pinças ópticas podem ser aplicadas a uma grande gama de estudos em diversas áreas, como biologia, medicina, arqueologia, nanoengenharia, entre outras, sendo necessária sua utilização por profissionais de diferentes formações. Dentre as desvantagens da pinça óptica comercial encontram-se o preço, além da dificuldade em acoplá-la a outros instrumentos que podem trabalhar em conjunto com a pinça. Esses pontos estimularam nosso grupo de pesquisa a desenvolver esse estudo e realizar a montagem de um arranjo próprio de pinça. 


\subsection{Nossa pinça}

Na Figura 4.1 temos o esquema experimental da pinça óptica montada nesse trabalho. Primeiramente, o feixe é expandido por um sistema de lentes biconvexas, sendo a primeira de $1.5 \mathrm{~cm}$ e a segunda de $5 \mathrm{~cm}$ de distância focal, respectivamente. A primeira encontra-se acoplada a um suporte com carrinho que permite movê-la em y e $z$, e, a segunda mantém-se fixa em um suporte que permite movê-la em $x, y$.

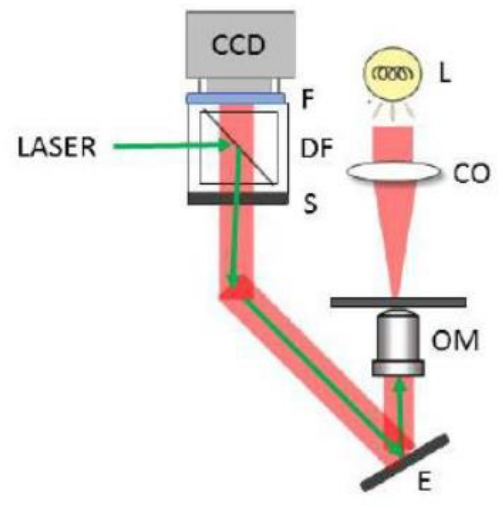

(a)

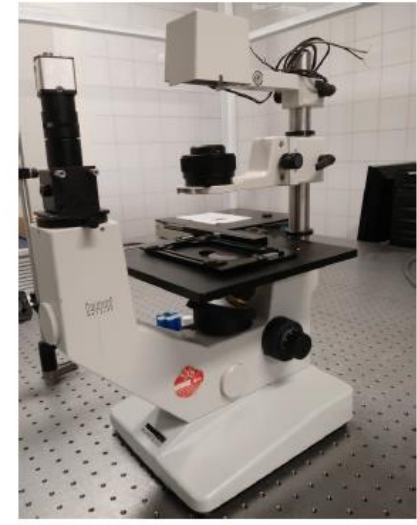

(b)

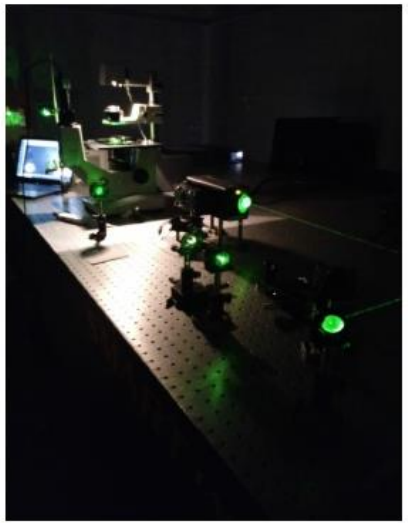

(c)

Figura 4.1: Diagrama da montagem da pinça óptica. O laser tem sua direção representada pela seta verde em (a). Este entra pela direita no cubo divisor de feixes e passa pelo prisma, sendo então refletido pelo espelho E para a objetiva OM. Esta permite a focalização do feixe no plano da amostra. Uma porção do feixe é refletida na superfície inferior da lamínula que contém a amostra e chega até a câmera CCD. Seguindo esse mesmo caminho, a luz branca segue para a câmera CCD, posicionada acima do cubo divisor de feixes. Neste esquema, CO corresponde à condensadora e L à lâmpada que ilumina a amostra. Em (b), temos a imagem do microscópio utilizado para a montagem e em (c), temos a imagem da pinça em funcionamento.

Posteriormente, o laser segue caminho até chegar a um cubo divisor de feixes que o desvia para um prisma presente no interior do microscópio adaptado. Um espelho contido no interior do microscópio o direciona para a objetiva de 100 x (Zeiss Jena), onde é focalizado no plano da superfície da amostra. Acima da amostra há uma condensadora e uma fonte de iluminação (lâmpada de $200 \mathrm{~W}$ ). A luz proveniente dessa fonte faz o percurso inverso ao do laser até o cubo e o atravessa até chegar a um tubo contendo um polarizador, uma lâmina retardadora, um filtro para luz verde e uma câmera DC Thorlabs conectada via USB a um computador que possui o software us 480 viewer, que serve para operar a câmera. Os filtros e 
o polarizador são utilizados para diminuir a incidência da luz proveniente do laser em relação à luz da lâmpada, para uma melhor observação da amostra. Para mover a amostra, adaptamos ao sistema mecânico já existente no microscópio um sistema de controle por motor de passo. Esse sistema permite o controle micrométrico nas direções do plano da amostra. A movimentação em $z$ é feita através dos parafusos de controle do microscópio. O sistema de movimentação controlado por um microntrolador ATmega 328 (plataforma IDE do Arduino) permitiu controlar a velocidade de deslocamento da microesfera em de mícron por segundo. Essa adaptação será abordada posteriormente na Secção 4.4.3.

Para comportar as microesferas, usamos vários modelos de suporte, no entanto, o suporte com melhor resultado foi uma lamínula com um anel plástico preso a ela com esmalte. Esse anel permitia a criação de um poço no qual era possível mover um pouco em $z$ as microesferas, ajudando a focalizá-las. Como as lamínulas são muito pequenas para que o suporte do microscópio pudesse movê-las, utilizamos uma impressora 3D para criar um retângulo com um orifício no meio e com outro nas laterais da espessura da lamínula. No entanto, não conseguimos prendê-la, pois a lamínula era curvada pela objetiva imersa em óleo, o que obrigou a colocação de fitas adesivas em forma de uma pirâmide para colar a lamínula, de modo que as fitas funcionassem como um amortecedor para quando a objetiva colasse na lamínula a impedia de curva-se. Em nossos experimentos utilizamos 6 fitas de comprimento 10,7 e $5 \mathrm{~cm}$, dobradas ao meio e coladas nas duas extremidades da cavidade da lamínula. A seguir, estão apresentadas fotos do aparato como um todo e do suporte feito na impressora com as fitas (Figuras 4.2 a 4.5 ).

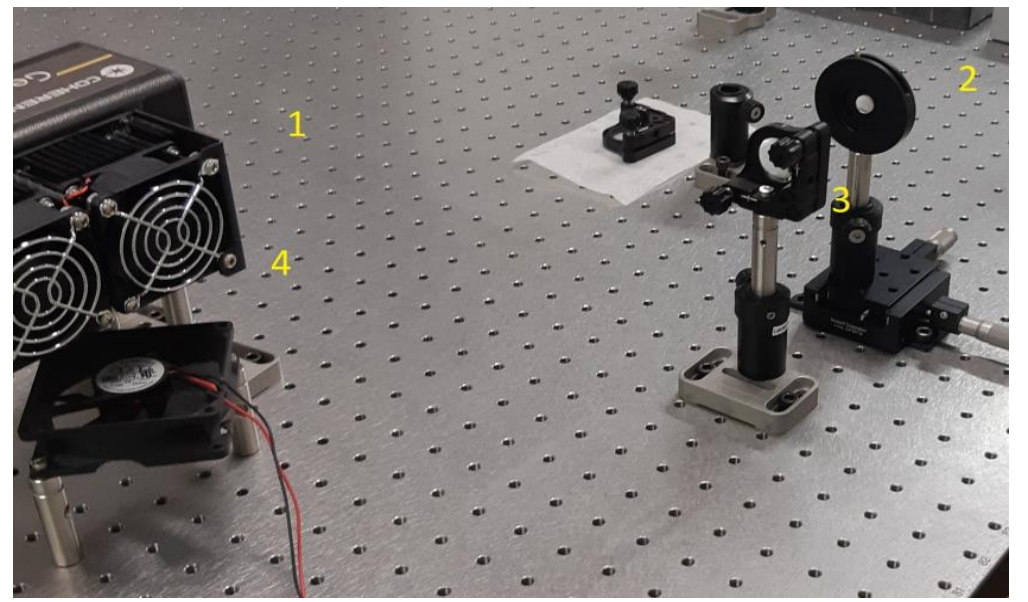

Figura 4.2: O número (1) indica o laser, (2) e (3) são as lentes expansoras de $f 1$ e $f 2$ respectivamente, e (4) indica o cooler utilizado para resfriar o laser. 


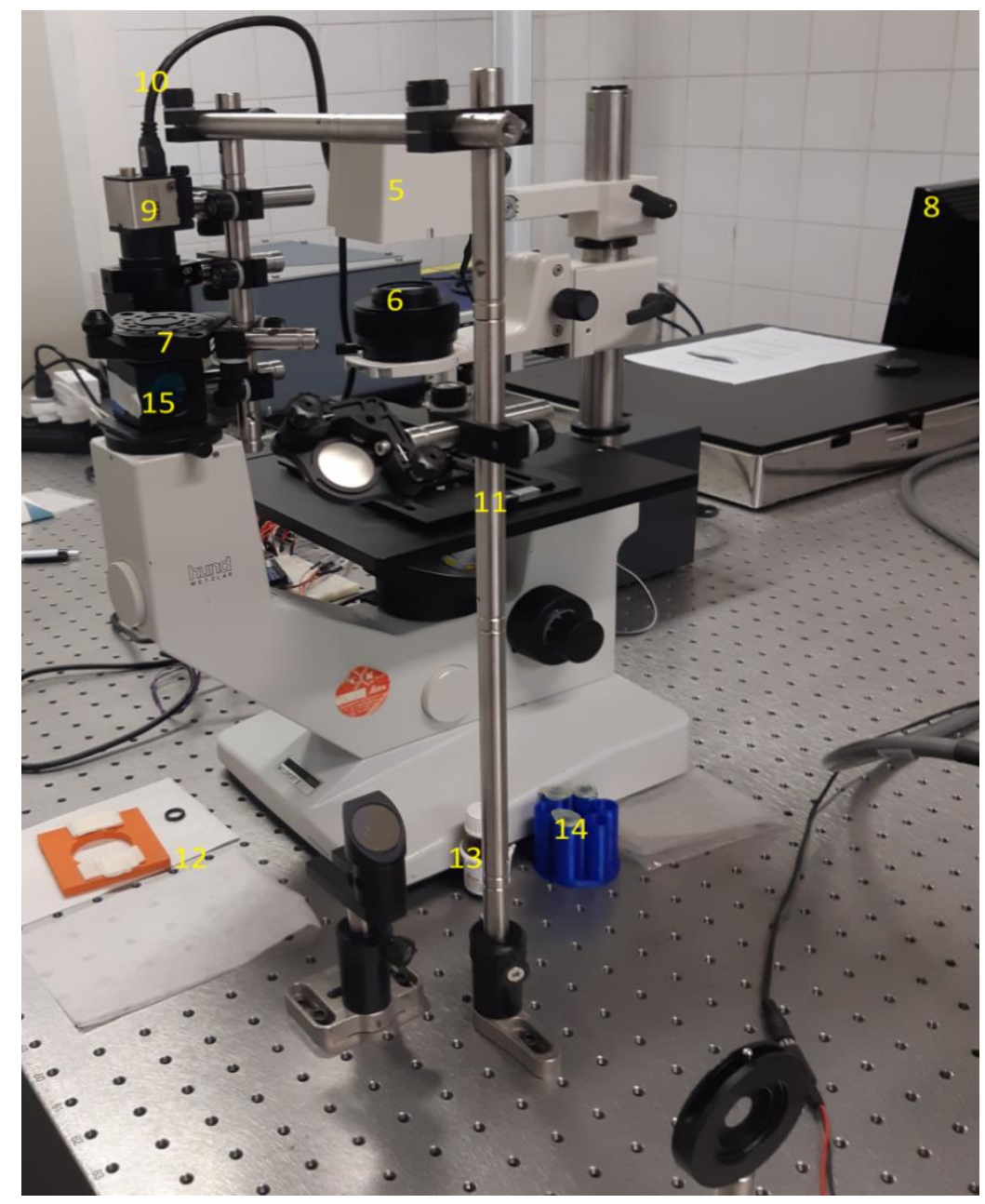

Figura 4.3: O número (5) indica a lâmpada que ilumina amostra; (6) a condensadora; (7) indicam polarizador e a lâmina de $1 / 4$ de onda que, juntos, permitem bloquear o laser; (8) o computador que controla a potência da luz; (9) a câmera com os filtros de luz ligada ao notebook; (10) o cabo USB que liga a câmera no notebook; (11) o suporte onde estão os espelho M3 e M4; (12) o suporte que utilizamos como porta amostras; (13) o óleo de imersão; (14) a solução de microesferas; (15) o cubo divisor de feixes. 


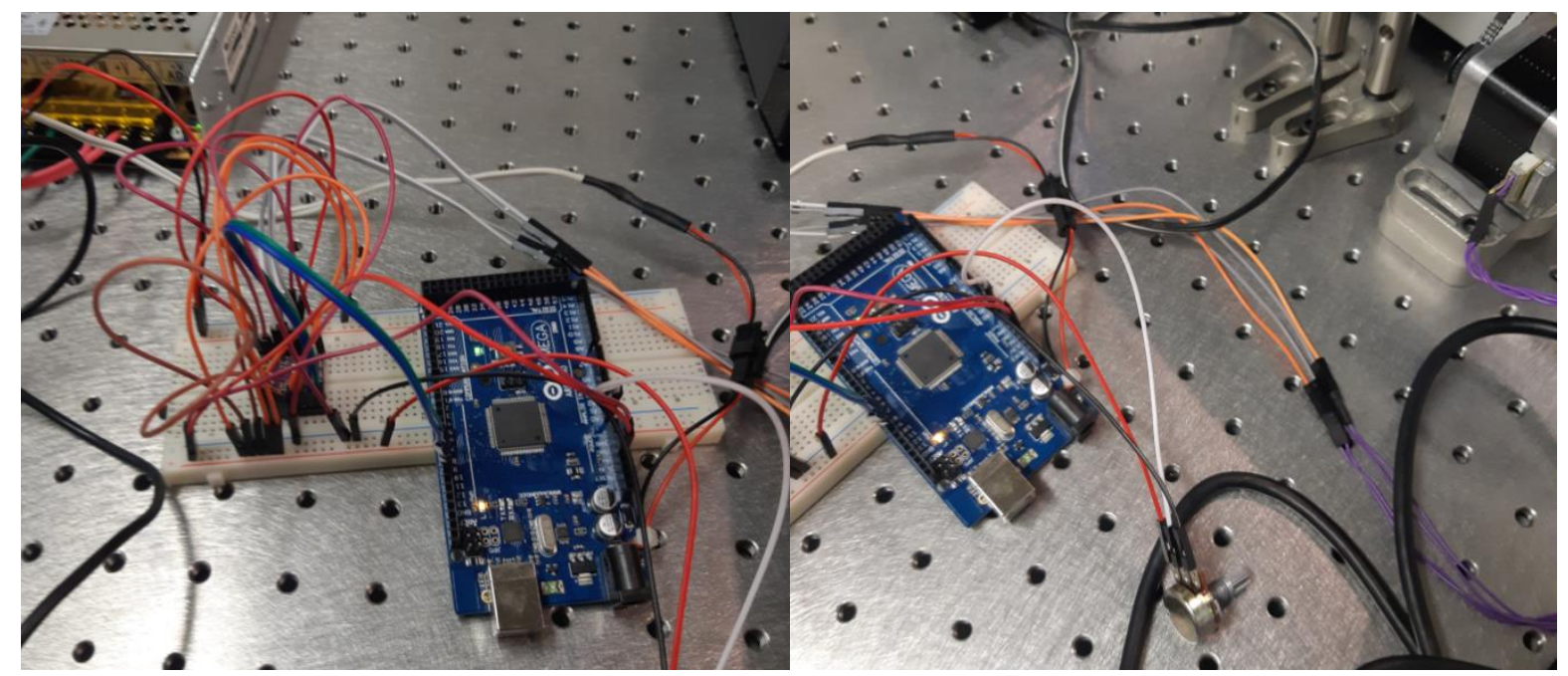

Figura 4.4: As imagens mostram: à esquerda, o microcontrolador utilizado no sistema de controle de posição da amostra e, à direita, o microcontrolador ligado ao motor de passo

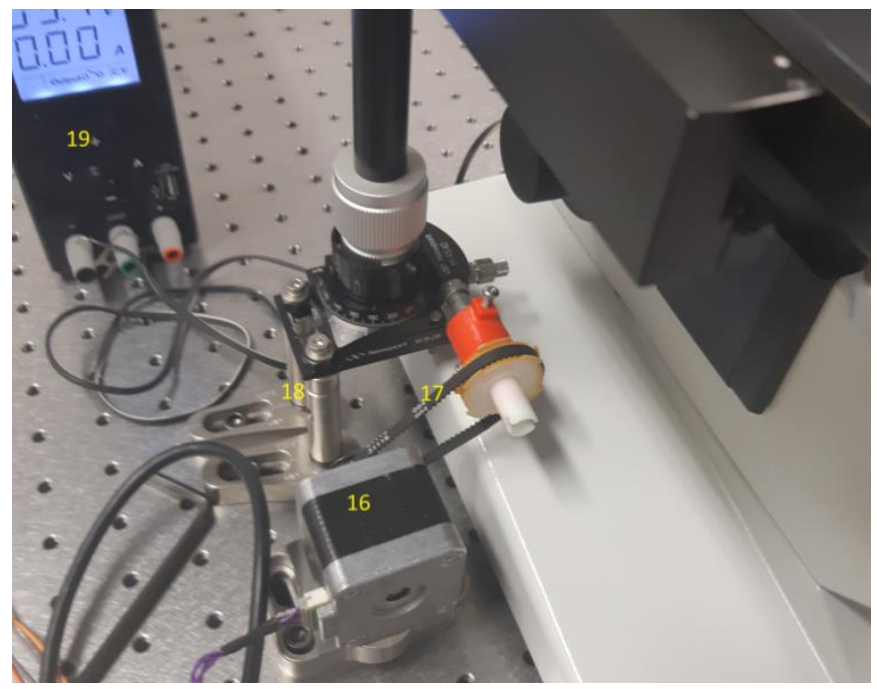

Figura 4.5: (16) motor de passo conectado ao microcontrolador através de um fio; (17) correia presa a um suporte plástico feito em impressora 3D. Esse suporte foi encaixado no parafuso micrométrico para rotacionar a alavanca que move a platina do microscópio; (18) suporte que conecta (17) à mesa óptica. (19) Fonte utilizada em ambas às montagens para alimentar a lâmpada que ilumina a amostra. Esse motor de passo foi adaptado ao sistema pelo técnico de nosso laboratório, Antônio Bloise, e a pós-doc Isis Brito. Através dele, podemos ter certeza de que a velocidade será a mesma no deslocamento das microesferas pela pinça óptica. 


\subsection{Método de montagem e problemas que podem ocorrer}

Para facilitar a explicação, os procedimentos experimentais foram apresentados em itens. Esses procedimentos citam as distâncias representadas na Figura 4.10.

(i) Inicialmente o laser foi fixado sobre uma messa óptica com sistema de amortecimento de vibrações. Aposição acima da mesa foi medida e marcada para ser utilizada como referência para alinhamento. Também tomamos o cuidado para que o laser sempre atingisse o centro dos espelhos para que não houvesse variação do ângulo de inclinação do feixe de forma a não alterar o formato final da matriz óptica.

(ii) Em seguida, o laser passa pelas lentes $f 1$ e $f 2$, de distâncias focais $1,5 \mathrm{~cm}$ e $5 \mathrm{~cm}$, respectivamente. Essas lentes foram posicionadas de forma a manter a distância de 6,5 cm uma da outra, ou seja, elas foram mantidas a uma distância que correspondia à soma das suas distâncias focais. A lente $f 1$ está sobre um carrinho e é possível movimentá-la em relação aos eixos z e y. A lente $f 2$ está fixada a um suporte fixo que permitia ajustes, sendo possível movê-la em relação ao eixo x,y. Usamos essas duas lentes para calibrar o tamanho do feixe adequado para prender as microesferas.

(iii) Posteriormente, alinhamos o laser de forma que o mesmo percorresse as distâncias D5, D6 e D7, que são mostradas na Figura 4. 7 da Secção 4.6. O feixe de luz sempre deve atingir o centro dos espelhos, pois caso ele atinja outro local, ele será refletido com ângulo de inclinação diferente do desejado, e isso fará com que pinça não funcione corretamente. Durante este processo, sempre utilizamos a altura de referência para determinar a direção do laser: altura em que o laser deve chegar no divisor de feixe que direciona o laser para o objetiva.

(iv) Para um alinhamento ainda mais preciso do laser, colocamos um espelho no lugar da amostra, ou seja, acima da objetiva, e o alinhamos de forma que o feixe refletido pelo espelho esteja na mesma posição do feixe que deixa o sistema de lentes. Posteriormente, só ajustamos a posição do laser pelo espelho M4 (Figura 4.7, Seção 4.6), pelo prisma e pela posição da lente $f 1$, pois queremos restringir ao máximo os graus de liberdade. Depois, marcamos um ponto de referência no porta amostras para conhecer o alinhamento da objetiva. Para tanto, movemos a objetiva no eixo $z$, garantindo a centralização do feixe. 
(v) Por fim, adicionamos uma pequena gota de óleo de imersão sobre a objetiva e a deslocamos até formar uma fina camada entre a saída da objetiva e uma lamínula $\mathrm{n}^{\circ}$ 0. Uma porção do feixe será transmitida pela amostra, mas uma parte será refletida pela primeira superfície da lamínula. Essa reflexão é direcionada à CCD e atenuada utilizando um sistema de polarizadores e lâmina retardadora. Além de um filtro próprio para o comprimento de onda do laser. Para garantir o alinhamento, conferimos se o padrão de reflexão se mantém circular à medida que dês focalizamos a lente. Para a armadilha óptica funcionar, o padrão de reflexão tem que ter o menor diâmetro possível. Isso pode ser conseguido ajustando de forma sutil a distância entre as lentes $f 1$ e $f 2$, o que permite alterar a divergência do feixe no plano de entrada da objetiva. Por fim, ligamos a lâmpada de iluminação e colocamos uma pequena gota de solução de microesferas de poliestireno, sendo possível ver através do computador que a amostra estava presa pela armadilha óptica.

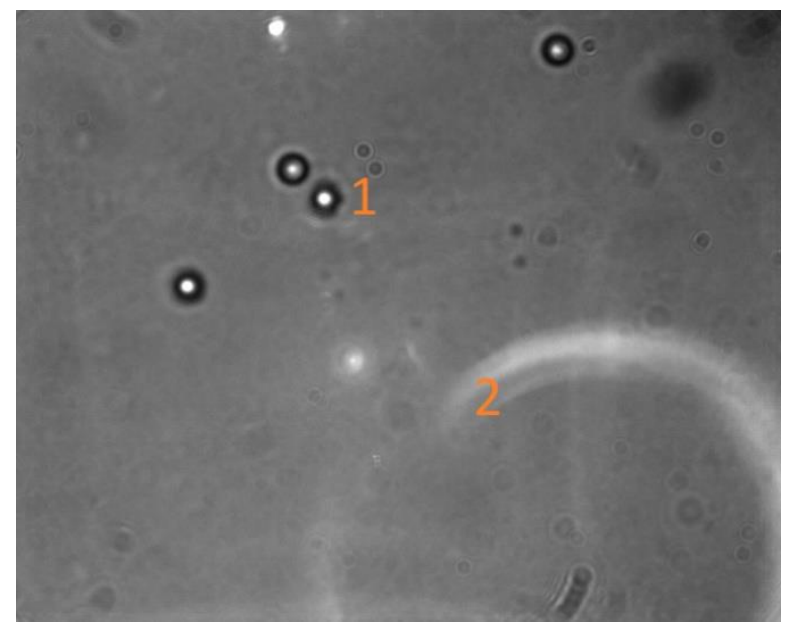

Figura 4.6: O número (1) indica a micro esfera aprisionada e (2) indica a reflexão não desejada do laser.

Os problemas que estão relacionados a cada um dos processos acima descritos são:

(1) Não se pode esquecer que todo o percurso o laser deve estar em linha reta e rente, estando os feixes sempre em paralelo. Qualquer alteração faria com que o laser entrasse com um ângulo diferente do eixo da objetiva e, assim, teríamos um ponto desfocalizado.

(2) A distância focal das lentes utilizadas deve ser pequena, ou seja, a distância entre as duas lentes deve ser pequena em comparação ao resto do caminho. Isso permite que uma pequena variação da distância entre as lentes gere uma maior variação na 
divergência do laser. O professor Dr. Carlos Lens, da UNICAMP foi essencial nessa etapa, pois nos orientou a utilizar os tipos certos de lentes.

(3) Todos os espelhos e lentes devem estar adequadamente limpos para que não haja interferência na armadilha.

(4) O cubo gera reflexões indesejadas na superfície da câmera. O sistema de polarizador e lâmina retardadora permite atenuar essa reflexão. Esse processo é essencial, pois a única porção de feixe que deve ser observada na imagem é a reflexão do laser na primeira face da lamínula.

(5) Como o laser tem uma intensidade e potência muito maior do que a iluminação da amostra pela luz da lâmpada torna-se difícil observar as microesferas presas pela armadilha. Por isso, adicionamos um filtro que permite atenuar a luz na faixa do comprimento de onda do laser. A lâmpada utilizada deve, junto com a condensadora, possibilitar uma imagem clara da amostra.

(6) A temperatura da sala deve ser baixa durante a realização dos experimentos, pois o laser e a lâmpada aquecem a água, aumentado muito o movimento das microesferas. O processo de aprisionar a amostra se torna ainda mais difícil se a temperatura da sala estiver acima de $25^{\circ} \mathrm{C}$. A temperatura ideal indicada é abaixo $\operatorname{dos} 20^{\circ} \mathrm{C}$.

(7) Se a intenção é fazer a captura das microesferas, devemos lembrar que a solução não pode ser muito concentrada, pois haverá muito espalhamento de luz devido ao grande número de microesferas no fundo do poço. Além disso, seu movimento freqüentemente faz com que elas grudem umas nas outras. Ao contrário, uma solução muito diluída gera dificuldade em encontrar microesferas. Além disso, com passar do tempo, a água em que a amostra está suspensa vai secando e essas micro esferas acabam se prendendo no fundo. Para resolver esses problemas foram preparadas várias soluções para serem testadas e, também, foi adicionada água após 15 minutos de exposição para repor aquela que evapora. Após vários testes, encontramos um volume médio ideal de solução, da ordem 30 microlitros. 


\subsection{Calibrações em x, y ou calibração radial.}

Para realizar a calibração radial, inicialmente adaptamos um estágio circular móvel encaixado a uma tampa e o colamos a um dos modos da alavanca que movimenta o suporte do microscópio em $x$. Para realizar a calibração usamos 3 métodos ${ }^{[4][5]}$ diferentes, cada um deles será descrito individualmente a seguir.

\subsubsection{Calibrações radiais com velocidade de escape}

Para realizar a calibração da velocidade de escape, prendemos uma bead e embutimos a ela uma velocidade utilizando o sistema de deslocamento a motor de passo. A amostra é movida, mas uma das microesferas permanece presa pela armadilha até o momento em que ela se solta. Todo o processo é filmando e gravado em um computador. O motor de passo permite imprimir uma velocidade constante, a qual foi determinada através do software livre Tracker. Usando a taxa de captura da câmera como referência, calculamos e velocidade de escape e, através dela, pudemos inferir a força através da equação Stokes para fluídos.

$$
6 \pi \mu R v_{\text {escape }}=F_{\text {optica }}
$$

Onde $\mu$ representaa viscosidade da água, $R$ o raio da microesfera, e $v_{\text {escape }}$ a velocidade de escape determinada através da análise anterior. Desta forma, através desses valores podemos determinar a força óptica em função da velocidade de escape para diferentes potências. Realizamos esse procedimento para $9 \mathrm{~mW}, 50 \mathrm{~mW}, 67 \mathrm{~mW}$ e $100 \mathrm{~mW}$. Esses dados serão apresentados no Capítulo 5.

\subsubsection{Calibrações radiais com movimento quadrado médio}

Para potências de ordem inferior a $30 \mathrm{~mW}$, observamos o efeito do movimento Browniano, que possui equação de energia igual, em valor numérico, à energia livre de Helmhotz para essa mesma partícula.

$$
\langle H\rangle=(1 / 2) k_{b} T
$$

onde $k_{b}$ representa a constante de Boltzmann e $T$ a temperatura do fluído. Desta maneira,essa seria a energia da microesfera na água. A energia associada à força óptica pode ser aproximada por um potencial harmônico nas regiões próximas ao centro do foco, o que nos permite escrever que, para uma partícula presa com movimento Browniano que tem suas energias equivalentes, tem-se:

$$
\langle H\rangle=\left(\frac{1}{2}\right) k_{b} T=\left(\frac{1}{2}\right) k\left\langle x^{2}\right\rangle
$$


Sabendo a temperatura e a velocidade quadrática mediana, podemos determinar a força óptica:

$$
F_{\text {optica }}=k x
$$

Para esse cálculo experimental, utilizamos a câmera para filmar durante algum tempo o movimento Browniano das microesferas e também utilizamos um programa no "Mathemática", de minha autoria, que altera a coloração das esferas para preto com bordas bem definidas (mais informações sobre esse programa podem ser encontradas no Apêndice F). Depois de tratarmos as imagens, as colocamos em ordem numérica e utilizamos o software de autoria do prof. Dr. Adriano M. Alencar, escrito em $\mathrm{C}++$, para determinar a posição em pixel, frame a frame, do centro da microesfera. Por fim, saliento que a temperatura ambiente foi medida em $(21,60 \pm 0,07){ }^{\circ} \mathrm{C}$, aferida com termômetro digital, após a espera de 10 minutos para que fosse atingido o equilíbrio térmico.

\subsubsection{Calibrações radiais com movimento quadrado médio}

Outro método de análise ${ }^{[4]}$ utilizado a partir desses dados foi encontrar a expressão da distribuição de Maxwell, onde as distribuições das posições medidas terão um padrão gaussiano, como podemos ver nas Gaussianas apresentadas no Capítulo 5. Desta forma, através de um programa, ajustamos a melhor função de probabilidade de Maxwell-Boltzmann onde as moléculas de uma partícula em um fluído térmico estão de acordo com a estatística de Boltzmann e se movimentam num caminho livre aleatório. Desta maneira, a colisão dessas moléculas com a bead faz com que elas adquiram o mesmo movimento, assim, podemos aplicar a mesma equação de distribuição de probabilidade para seu movimento errático. Sendo equação de Maxwell-Boltzmann:

$$
\rho(r)=\rho_{0} \int \exp \left[-\frac{U(r, x, y)}{k_{b} T}\right]
$$

onde $r^{2}=x^{2}+y^{2}$ e $\rho_{0}=\frac{1}{\int \exp \left[-\frac{U(r, x, y)}{k_{b} T}\right]}$ são constantes de renormalização da equação de

probabilidade; sendo que $U(r, x, y)=m \frac{r^{2}}{2}+F(x, y)$ e representa a energia mecânica, onde a derivada de $r$ é muito pequena dado o aprisionamento ótico,então a desprezamos. Além disso, como já mencionado, as forças ópticas próximas ao centro da armadilha podem ser aproximadas pela Lei de Hooke onde: $F(x, y)=-\left(k_{x} x^{2}+k_{y} y^{2}\right)$. A partir dessa relação, podemos chegar na constante da armadilha óptica, só que de maneira mais apurada pois, cada medida está associada ao erro intrínseco de sua posição, a qual somaremos os erros pelo 
método da velocidade quadrática média. Isso nos leva a concluir que, através desse método, temos uma melhor aproximação do valor real da constante.

\subsubsection{Calibrações radiais através da força de Stokes}

Essa calibração baseia-se nas Equações 4.1 e 4.4, onde, conhecendo um deslocamento $x$, quando a partícula está em movimento sofrendo a influência da força de Stokes da água a uma determinada velocidade, podemos determinar sua força. Sabemos a velocidade da partícula, pois esta é a mesma dada pelo motor de passo, e, através da medida desse deslocamento, com a utilização de software, podemos determinar a força de Stokes. A partir dos frames gravados, usando um programa no "Mathematica", podemos determinar esse deslocamento e, com esta informação, podemos usar Equação 4.1 e determinar a constante $k$ para diferentes potências do laser. Detalhes sobre esse programa estão descritos no Apêndice G.

\subsection{Calibração no eixo z}

Não foi possível realizar a calibração em $z$, pois não tínhamos os equipamentos necessários, como a capacidade de mover livremente a amostra em z, além de não possuirmos outro laser para observar microesferas fluorescentes e, assim, detectar o deslocamento axial no poço. No entanto, iremos explicar o processo estudado ${ }^{[1][4]}$, onde uma microesfera fluorescente é acompanhada por um fotodetector que rastreará a emissão de florescência em diferentes planos focais. Desta forma, a partir do uso desse detector e do motor de passo aplicado ao deslocamento em $z$, podemos utilizar os mesmos métodos da detecção radial.

\subsection{Representação em matriz dos componentes ópticos da pinça}

A representação matricial óptica é um meio eficiente para se determinar o ângulo e o tamanho final de feixe de luz, sendo usada com frequência no ramo da óptica. Nesse texto, irei representar, em forma de matriz, os componentes da pinça óptica e determinar através dessa mesma matriz, seu ângulo e comprimento final. Esse método, usado para raios paraaxiais,é freqüentemente chamado método $\mathrm{ABCD}$. Nesta apresentação utilizarei referência de SALEH(1991) $)^{[2]}$, onde um feixe paraxial ao passar por um componente ótico como lente, espelho, etc., interage com o feixe mudando seu tamanho e ângulo:

$$
\left(\begin{array}{l}
y_{1} \\
\theta_{1}
\end{array}\right)=\left(\begin{array}{ll}
A_{1} & B_{1} \\
C_{1} & D_{1}
\end{array}\right)\left(\begin{array}{l}
y_{0} \\
\theta_{0}
\end{array}\right)
$$


Onde os componentes da matriz são constantes que variam de acordo com os componentes representados.Vale lembrar que esse método funciona para raios paraxiais, onde $\theta \approx \operatorname{sen} \theta$, e, considerando que o feixe terá mudança nas condenadas $(x, y)$, estes sistemas ópticos possuem mais variáveis além das coordenadas espaciais. Como nosso sistema não necessita dessa complexidade matemática, não iremos detalhá-las.Logo, se o feixe passa por dois componentes teremos que:

$$
\left(\begin{array}{l}
y_{2} \\
\theta_{2}
\end{array}\right)=\left(\begin{array}{ll}
A_{1} & B_{1} \\
C_{1} & D_{1}
\end{array}\right)\left(\begin{array}{ll}
A_{2} & B_{2} \\
C_{2} & D_{2}
\end{array}\right)\left(\begin{array}{l}
y_{0} \\
\theta_{0}
\end{array}\right)
$$

Onde teremos um novo feixe com comprimento e ângulo de incidência final $\left(y_{2}, \theta_{2}\right)$ logo, expandindo para $\mathrm{n}$ componentes teremos:

$$
\left(\begin{array}{l}
y_{n} \\
\theta_{n}
\end{array}\right)=\prod_{i=1}^{n}\left(\begin{array}{cc}
A_{i} & B_{i} \\
C_{i} & D_{i}
\end{array}\right)\left(\begin{array}{l}
y_{0} \\
\theta_{0}
\end{array}\right)
$$

Desta forma,a pinça óptica possui os seguintes componentes e percurso do laser, representados na Figura 4.7.

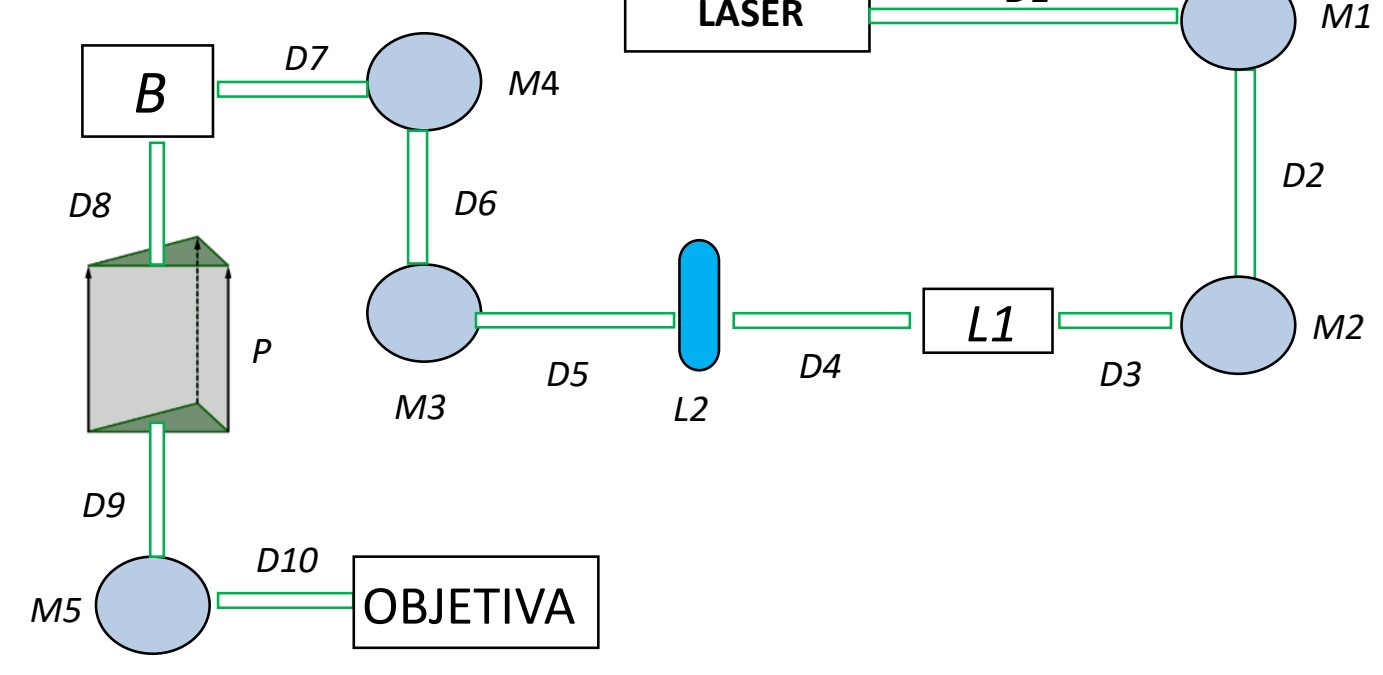

Figura 4.7: Representação do caminho óptico percorrido pelo laser montagens, onde os $M 1$ $M 5$ são espelhos que redirecionam o laser, $L 1$ a lente de $1.5 \mathrm{~cm}, L 2$ a de $5 \mathrm{~cm}, B$ cubo divisor de feixes, $P$ o prisma e a objetiva que focaliza a imagem da amostra. 
Na Figura 4.7 representamos o caminho óptico percorrido pelo laser. Nessa figura, M1, M2, M3, M4 e M5 representam espelhos planos. Os suportes desses espelhos possuem parafusos que permitem realizar ajustes finos em sua direção. Desta forma, por considerarmos que eles estão alinhados sem uma posição fixa, consideramos uma representação matricial de espelho plano. O laser passa por um sistema de lentes convergentes e continua até chegar no divisor de feixes B, passando, depois, pelo prisma $\mathrm{P}$ e, finalmente, atravessa a objetiva principal de 100x.

As formas matriciais são dadas ${ }^{[2]}$ para o deslocamento livre com ângulos pequenos determinado por $T(x)=\left(\begin{array}{ll}1 & x \\ 0 & 1\end{array}\right)$. Para a reflexão do espelho temos $M(x)=\left(\begin{array}{ll}1 & 0 \\ 0 & 1\end{array}\right)$. Para a objetiva, temos o tratamento para uma lente fina delgada que nos dá $o b j=\left(\begin{array}{cc}1 & 0 \\ -\left(\frac{1}{f}\right) & 1\end{array}\right)$. Isso vale para as outras lentes $L 1$ e $L 2$ com distâncias focais $f 1$ e $f 2$. A forma matricial para o cubo divisor de feixes é aproximada para $C=\left(\begin{array}{cc}1 & d 1 n_{\text {vidro }} \\ 0 & 1\end{array}\right)$, e, por fim, o laser passa pelo prisma cuja matriz aproximada é $P=\left(\begin{array}{cc}1 & d 2 n_{\text {vidro }} \\ 0 & 1\end{array}\right)$.

A matriz referente ao espelho plano é a matriz identidade. Por fim, a matriz foi calculada por um programa do "Mathematica" que está apresentado no Apêndice F.

$$
M=A 4 . A 3 \cdot A 2 \cdot A 1
$$

onde A1 tem o valor de:

$$
\begin{gathered}
A 1=T(D 2) \cdot T(D 1)=A 1=\left(\begin{array}{cc}
1 & D 1+D 2 \\
0 & 1
\end{array}\right) \\
A 2=T(D 5) \cdot L 1(f 2) \cdot T(D 4) \cdot O B J(f 1) \cdot T(D 3)= \\
\left(\begin{array}{cc}
1-\frac{D 5-D 4\left(1-\frac{D 5}{f 2}\right)}{f 1}-\frac{D 5}{f 2} & D 5+D 4\left(1-\frac{D 5}{f 2}\right)-D 3\left(1-\frac{D 5-D 4\left(1-\frac{D 5}{f 2}\right)}{f 1}-\frac{D 5}{f 2}\right) \\
-\frac{1-\frac{D 4}{f 2}}{f 1}-\frac{1}{f 2} & 1+D 3\left(\frac{1-\frac{D 4}{f 2}}{f 1}-\frac{1}{f 2}\right)-\frac{D 4}{f 2}
\end{array}\right) \\
A 3=T(D 6) \cdot T(D 7) \cdot B=\left(\begin{array}{cc}
1 & D 6+D 7 \\
0 & 1
\end{array}\right) \\
P=\left(\begin{array}{cc}
1 & k 1 n_{\text {vidro }} \\
0 & 1
\end{array}\right) ; C=\left(\begin{array}{cc}
1 & k 2 n_{\text {vidro }} \\
0 & 1
\end{array}\right)
\end{gathered}
$$

Onde $d 1$, representa a distância que o feixe percorre dentro do cubo divisor de feixes que é $2,54 \mathrm{~cm}$ e $d 2$ a distância que o feixe percorre no prisma que vale $1,92 \mathrm{~cm}$.

$$
A 4=T(D 8) \cdot T(D 9) \cdot T(D 10)=\left(\begin{array}{cc}
1 & D 18+D 9+D 10) \\
0 & 1
\end{array}\right)
$$


Desta maneira, chegamos na matriz final Mf, que pode ser consultado no Apêndice F. Assim, realizamos as medidas dos valores das distâncias utilizando fita métrica e obtivemos os seguintes valores:

Tabela 4.1: Valores das distâncias entre os componentes representados na Figura 4.7.

\begin{tabular}{cc}
\hline $\begin{array}{c}\text { Distância entre os } \\
\text { componentes }\end{array}$ & Valores das distâncias $(\mathrm{cm})$ \\
\hline D1 & 58,00 \\
D3 & 29,50 \\
D4 & 35,50 \\
D5 & 7,10 \\
D6 & 55,50 \\
$D 7$ & 20,50 \\
$D 8$ & 20,00 \\
$D 9$ & 15,50 \\
$D 10$ & 9,75 \\
\hline
\end{tabular}

Logo, sendo o diâmetro inicial do laser, segundo o manual, de $y_{0}=1.0 \mathrm{~mm}$ e $\theta_{0} \approx 0$ ( $\theta_{0}$ não é exatamente zero, mas uma valor bem menor que um radiano), e utilizando as Equações 4.8 e 4.14 com os valores calculados e mostrados na tabela acima, temos que:

$$
y_{0}\left(1-\frac{d 5+d 4\left(1-\frac{d 5}{f 2}\right)}{f 1}+(d 1+d 2)\left(-\frac{1-\frac{d 4}{f 2}}{f 1}-\frac{1}{f 2}\right)-\frac{d 5}{f 2}\right)=y_{14}
$$

Onde o número 14 representa as 14 matrizes multiplicadas que geram a matriz final. Nesse caso, $f 1=1,5 \mathrm{~cm}$ e, $f 2=5 \mathrm{~cm}$. $y_{14}=7.7066 \mathrm{~mm}$, cujo erro é maior que $10^{7}$, ou seja, temos uma grande perda de significância, sendo que o valor final medido foi $y_{\text {experimental }}=3.33 \pm 0.05 \mathrm{~mm}$. Desconsideramos a validade do resultado devido à perda de significância. Provavelmente, essa diferença foi resultado das várias aproximações que fizemos ao construir as matrizes. 


\section{Referências:}

[1] A. A. R. Neves. Força óptica em pinças ópticas: estudo teórico e experimental. 2006. 169 p. Tese (doutorado) - Universidade Estadual de Campinas, Instituto de Física Gleb Wataghin, Campinas, SP.

[2] B. E. A. Saleh, and M. C. Teich, Fundamentals of photonics. Wiley, 2ed., 2006.

[3]F. J. Duarte. Tunable Laser Optics. CRC Press, 2 ed., 2015.

[4]Z. J. Ulanowski, andI. R. Williams, “Optical tweezers”,Physics Education,vol. 31, no. 3, 1996.

[5] Fontes, A.. Microanálise com Pinças Ópticas, 2004, 271 p. Tese (doutorado)-Universidade Estadual de Campinas, Instituto de Física GlebWataghin, Campinas, SP. 


\section{Capítulo 5}

\section{Resultados e Análises}

\subsection{Resultado do experimento de máxima velocidade para força radial}

Como será descrito mais adiante, no experimento 5.3.1 realizamos a movimentação do suporte através da alavanca do microscópio acoplada a um motor de passo que nos permitiu manter uma velocidade aproximadamente constante. Essa velocidade foi determinada através da medida do deslocamento pelo tempo utilizando um software livre chamado "Tracker", disponível em: <https://physlets.org/tracker/>. Para 10 seções de vários vídeos, medimos o deslocamento das microesferas em instantes de 1 a 3 segundos. Os resultados das medidas estão na Tabela 5.1 e esses resultados correspondem ao segundo e ao último ponto, pois, como há oscilação, a velocidade mais correta será a com maior diferença entre os pontos. A partir desses resultados obtivemos uma velocidade média de 12,5 $\mu \mathrm{m} / \mathrm{s}$. Na Figura 5.1 temos a imagem retirada do software "Tracker" após a realização de uma das medidas dos 10 vídeos distintos.

Tabela 5.1: Valores da velocidade do motor obtido pelo software "Tracker" para diferentes vídeos

\begin{tabular}{cc}
\hline Tentativas & \multicolumn{2}{c}{ Velocidade da partícula em $\mu \mathrm{m} / \mathrm{s}$} \\
\hline 1 & 12,367 \\
2 & 12,955 \\
3 & 12,544 \\
4 & 11,548 \\
5 & 12,150 \\
6 & 13,666 \\
7 & 11,118 \\
8 & 11,911 \\
9 & 13,641 \\
10 & 13,175 \\
\hline
\end{tabular}




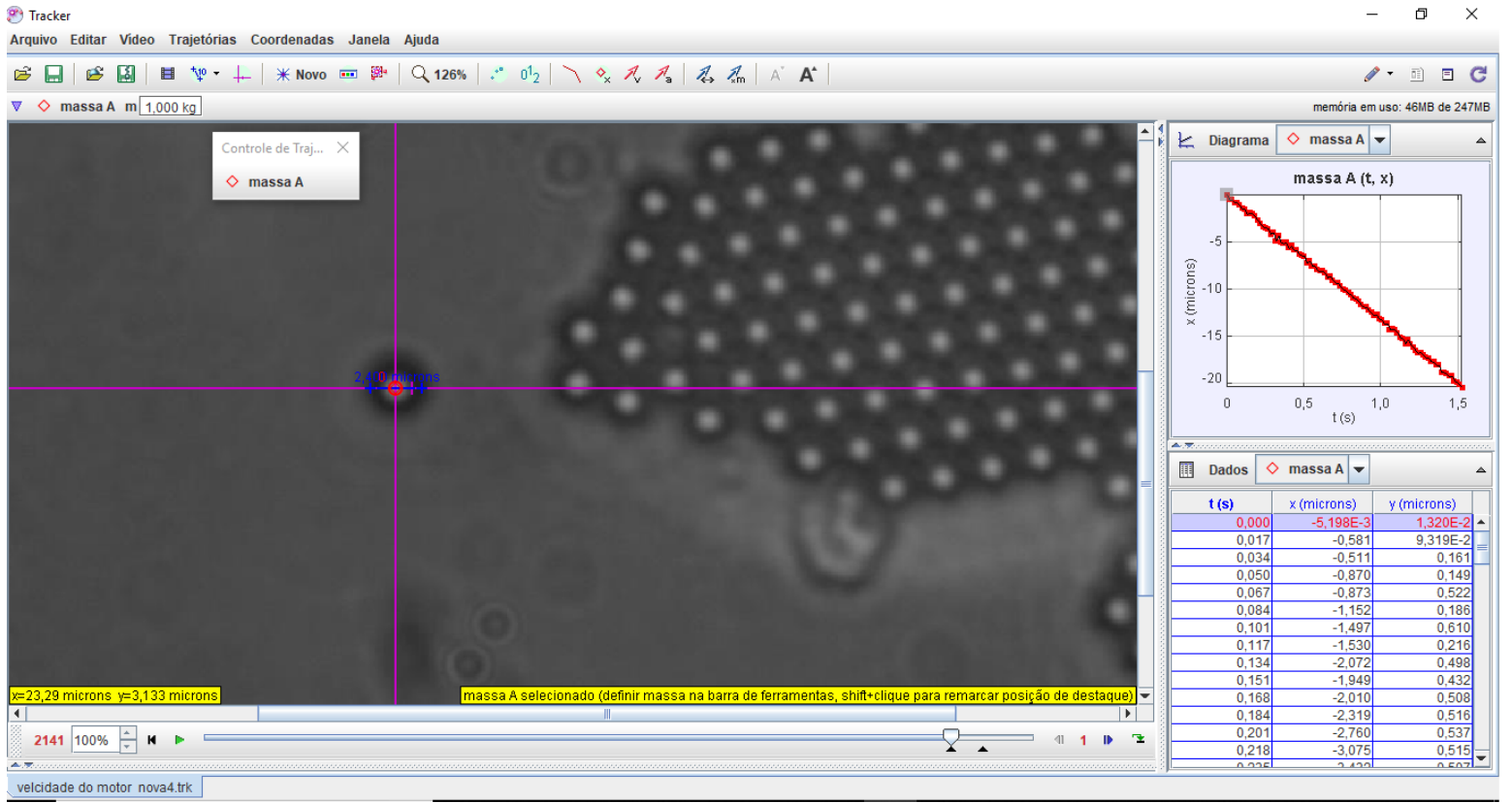

Figura 5.1: Tela de capturada do software "Tracker."A imagem mostra a bead analisada.

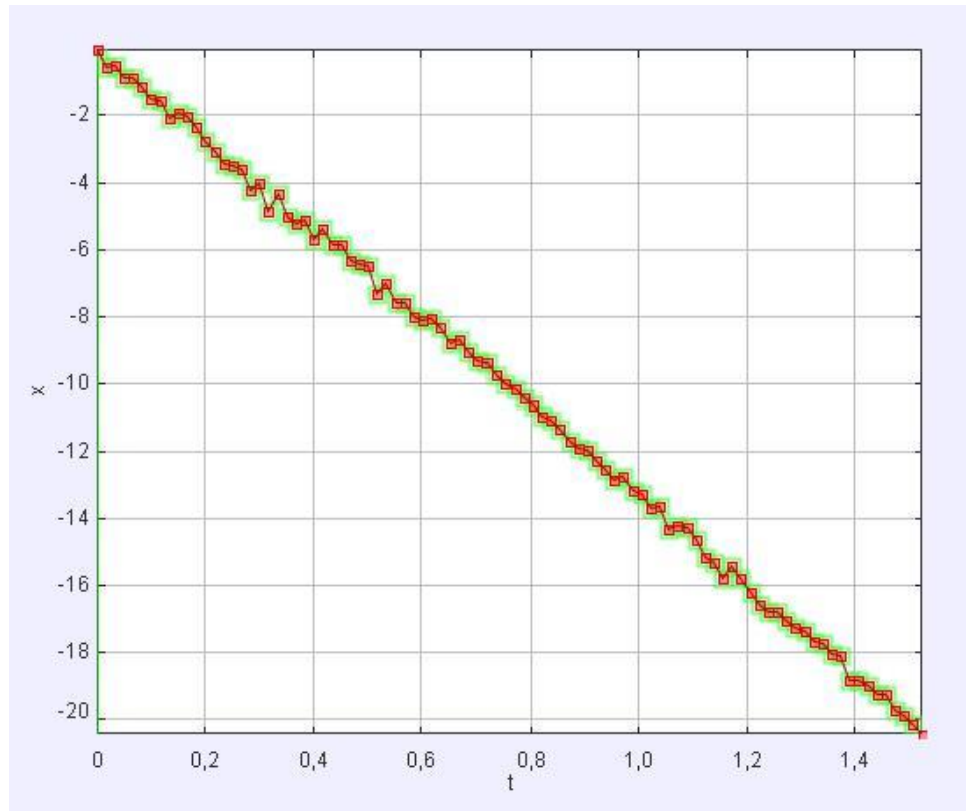

Gráfico 5.1: Gráfico da posição $(\mu m)$ pelo tempo $(\mathrm{s})$ da tentativa $\mathrm{n}^{\circ} 10$. A não suavidade da curva dá-se pelo tremor causado pelo motor de passo ligado ao sistema, sendo esse gráfico o que está representado na Figura 5.1.

Sabendo a velocidade da microesferas podemos aplicar a lei de Stokes (Equação 4.1), e, desta forma, temos que a força exercida sobre uma partícula de 1,2 mícrometro de raio 
imersa em água à temperatura de $294 K$ é de $(2.7 \pm 0,7) \cdot 10^{-13} \mathrm{~N}$. Nesse caso, o erro se dá pelo desvio padrão para o valor da velocidade somado ao erro do raio das microesferas, valor esse dado pelo fabricante.

Neste trabalho, a microesfera aprisionada foi movida utilizando diferentes potências do laser até o momento em que ela se desprendia da armadilha. Após a realização dos ensaios, percebeu-se que a potência em que isso ocorria correspondia a $9 \mathrm{~mW}$ na saída do laser e 2,1 $\mathrm{mW}$ na entrada da objetiva. Esse processo foi filmado diversas vezes para garantir o resultado aqui apresentado. Quatro medidas da velocidade foram retiradas desses vídeos, sendo este escape efetuado 12 vezes. Logo, sabendo a potência, foi possível comparar os gráficos simulados das Equações 2.132, com e sem deslocamento focal. Devemos salientar que a variação está nas funções dos coeficientes de forma da onda, que correspondem às Equações 3.19 a 3.22 e 3.25 a 3.28, tendo, essas últimas, as novas integrais 3.23 e 3.24. Comparamos essa medida da força com a força máxima de retenção da pinça óptica, a qual corresponde ao maior valor apresentado no gráfico.

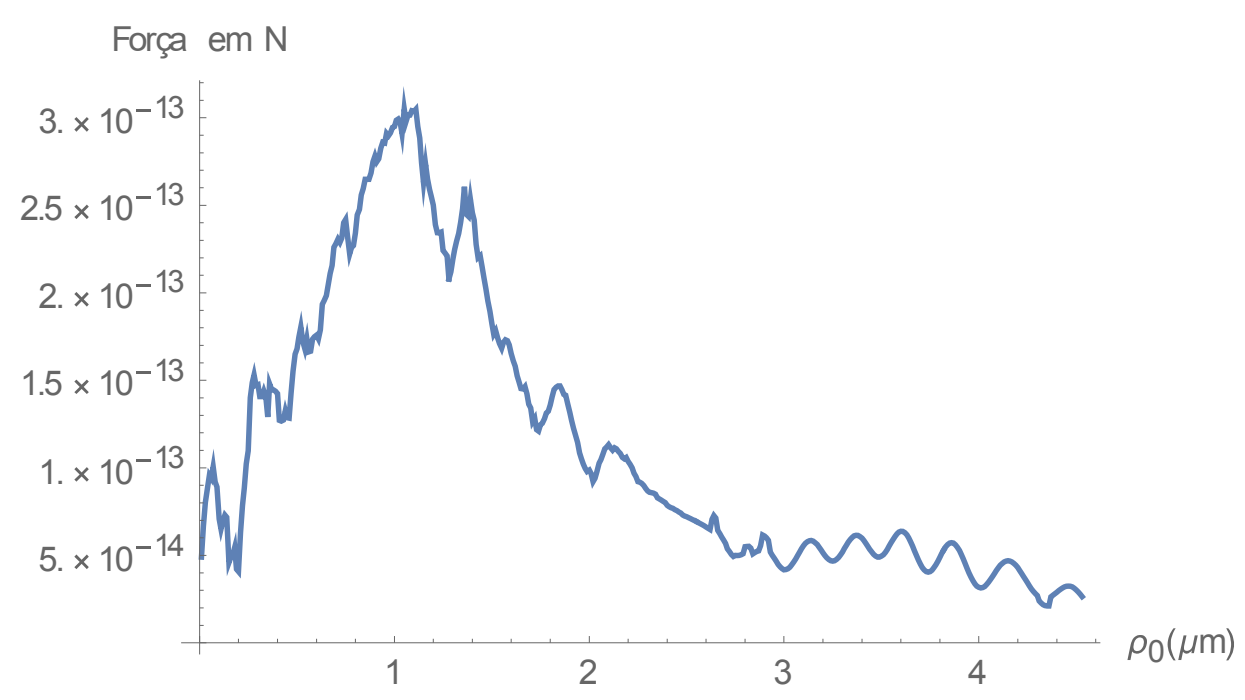

Gráfico 5.2: A força paralela em $x$ com deslocamento focal para a microesfera simulada. A força máxima é de $3 \cdot 10^{-13} \mathrm{~N}$, estando fora do intervalo de erro da força experimental de $(2.7 \pm 0,7) \cdot 10^{-13} N$. 


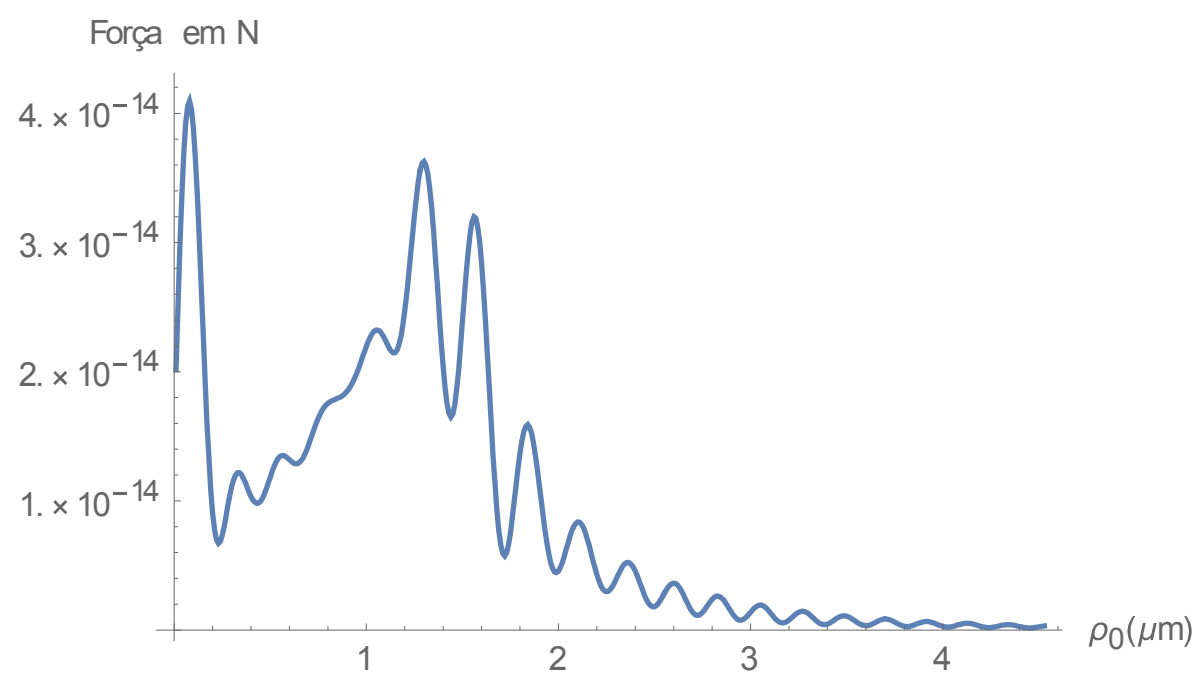

Gráfico 5.3: Gráfico da força sem deslocamento focal em relação ao eixo. A força máxima está próxima a $4 \cdot 10^{-14} N$.

Como podemos ver pelos resultados, a força simulada chega próximo ao valor experimental, mas não se mantém dentro da margem de erro. Durante a simulação, houve fatores que não foram considerados, como a perda por intensidade em relação à distância da lamínula e as forças de absorção e espalhamento gerados pela interação do laser com as microesferas. Outro ponto importante é o fato de que a matriz óptica $\mathrm{C}$ teórica é nula, diferente do que ocorre experimentalmente. Outra questão é a diferença, por mais que pequena, gerada pela adição do óleo de imersão na região entre a lente e a amostra. Assim, para uma melhor aproximação da força seria necessário adicionar esses elementos na simulação para estudos teóricos, além de tentar minimizar os erros experimentais descritos acima. Quanto à forma da força, os resultados estão de acordo com a literatura ${ }^{[1-5]}$.

\subsection{Resultado da simulação da força para o caso axial}

Apesar de não terem sido realizados experimentos para o eixo z, como mencionado no Secçâo 3.4, foram feitas as simulações para essa direção. Onde, sabendo que a força em $z$ tem relação de grandezas com radial ${ }^{[1][8]}$ podemos comparar com o valor obtido radialmente com, as simulações que estão apresentadas nos Gráficos 5.4 e 5.5. Vale salientar que, os fatores relacionados aos fenômenos que faltam implementar na simulação para caso radial, valem para caso axial. 


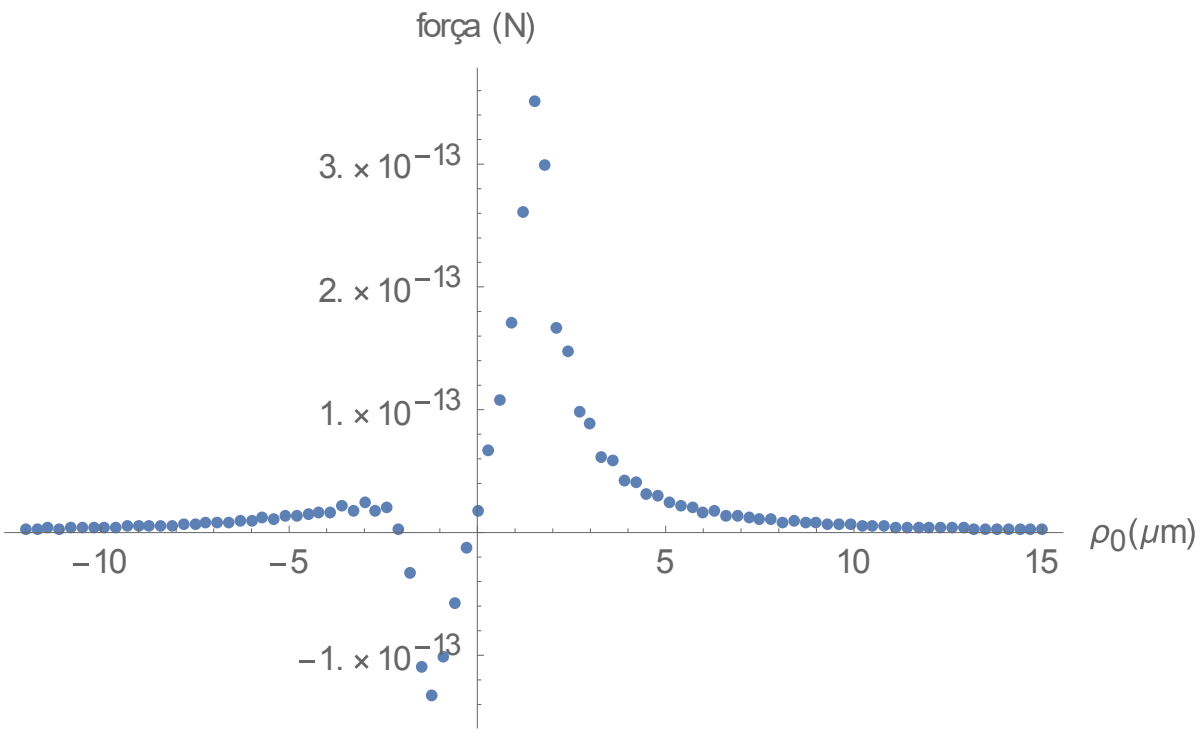

Gráfico 5.4: Simulação da força axial (Equação 2.131) sem deslocamento focal, sendo que a força é da ordem próxima à somatória das forças radiais.

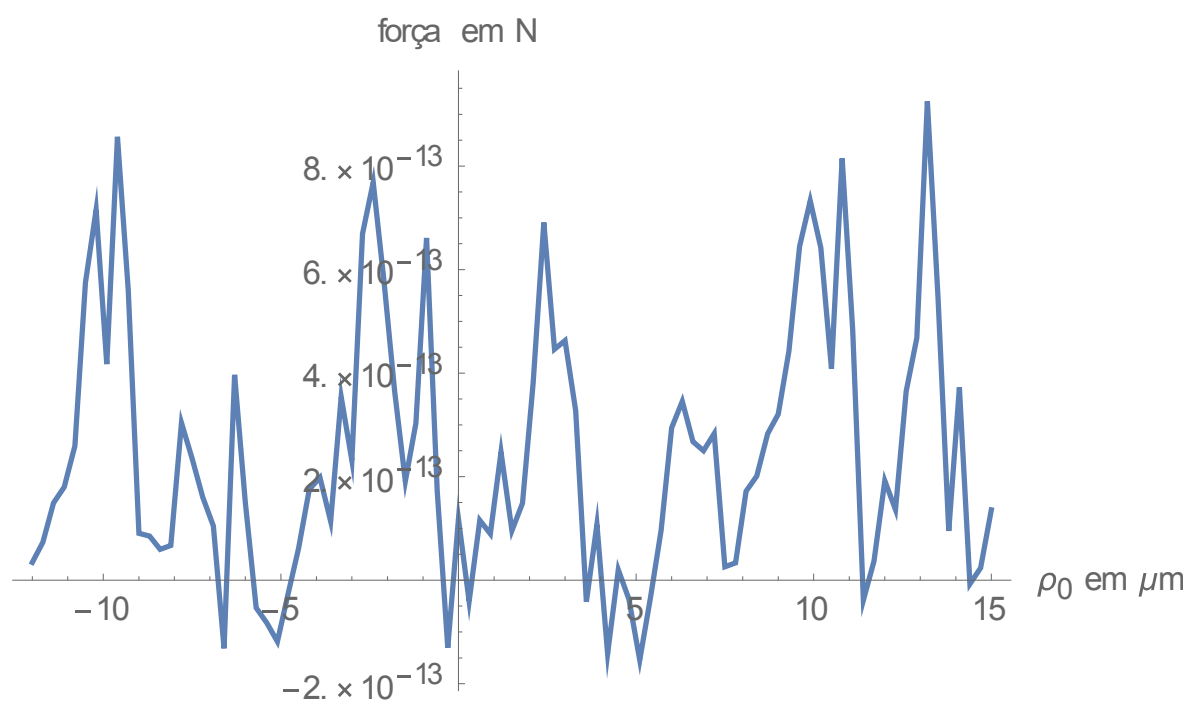

Gráfico 5.5: Força com efeito de deslocamento focal. Devido à alta variação de valores e overflow só foi possível simular o gráfico perto da origem onde, de acordo com a teoria, a força se comporta como reta com probabilidade de $k x$.

Desta maneira, a forma da onda axial sem deslocamento focal se amostra adequada com os dados presentes na literatura ${ }^{[1-5]}$. A forma axial com deslocamento focal também se adéqua ${ }^{[4]}$ com medidas feitas da intensidade elétrica pela distância obtidas em outros estudos. Quanto a seu valor máximo sabe-se ${ }^{[1],[8]}$ que, por causa do espalhamento, a força detectada 
experimentalmente na direção axial é de $10 \%$ ou $1 \%$ da em relação radial. Contudo, como não foi realizado nenhum experimento de medida na direção $\mathrm{z}$ por impedimentos técnicos, não é possível estimar se o verdadeiro valor da força é válido.

\subsection{Resultados do experimento para o movimento Browniano}

\subsubsection{Comparado à simulação em relação a $x$}

Como explicado na Secção 4.3.2, utilizamos as posições em $x, y$ e calculamos, a partir da temperatura, a constante dielétrica do feixe através de dois métodos. Primeiramente tratarei das coordenadas em $x$. Os resultados podem ser observados na Tabela 5.2.

Tabela 5.2: Estão apresentados os valores da potência do laser que prende as microesferas, sua posição quadrática média horizontal, seu $k_{x}$ calculado, o erro dado pelo ajuste na curva, e os valores de $k$ simulados com e sem o fenômeno do deslocamento focal.

\begin{tabular}{cccccc}
\hline $\begin{array}{c}\text { Potência } \\
(\mathrm{mW})\end{array}$ & $\begin{array}{c}<\Delta \mathrm{x}^{2}>\mathrm{MSD} \\
\mathrm{em} 10^{-14} \mathrm{~m}^{2}\end{array}$ & $\begin{array}{c}k_{x}(N / m) \\
\mathrm{x} 10^{-9}\end{array}$ & $\begin{array}{c}\text { Erro } \\
\text { de } k_{x}\end{array}$ & $\begin{array}{c}\left(k_{x} N / m\right) \times 10^{-9} \\
\text { simulado sem } \\
\text { desl. focal }\end{array}$ & $\begin{array}{c}\left(k_{x} N / m\right) \times 10^{-9} \\
\text { simulado com } \\
\text { desl. focal }\end{array}$ \\
\hline 0,695 & 1,837200 & 222 & 16 & 12,8417 & 96,0885 \\
1,195 & 1,110420 & 366 & 44 & 22,0803 & 165,2170 \\
1,655 & 1,006190 & 404 & 54 & 30,5799 & 228,8150 \\
2,100 & 0,865438 & 471 & 73 & 38,8023 & 290,3390 \\
2,565 & 0,825168 & 493 & 80 & 47,3942 & 354,6290 \\
2,980 & 0,638084 & 637 & 135 & 55,0623 & 412,0050 \\
3,410 & 0,527068 & 772 & 197 & 63,0075 & 471,4560 \\
3,470 & 0,487164 & 835 & 231 & 64,1161 & 479,7510 \\
4,890 & 0,481394 & 845 & 237 & 90,3539 & 676,0760 \\
5,700 & 0,277720 & 1470 & 732 & 105,3200 & 788,0640 \\
\hline
\end{tabular}

Para os pontos obtidos foi traçado o gráfico apresentado no Gráfico 5.6. 


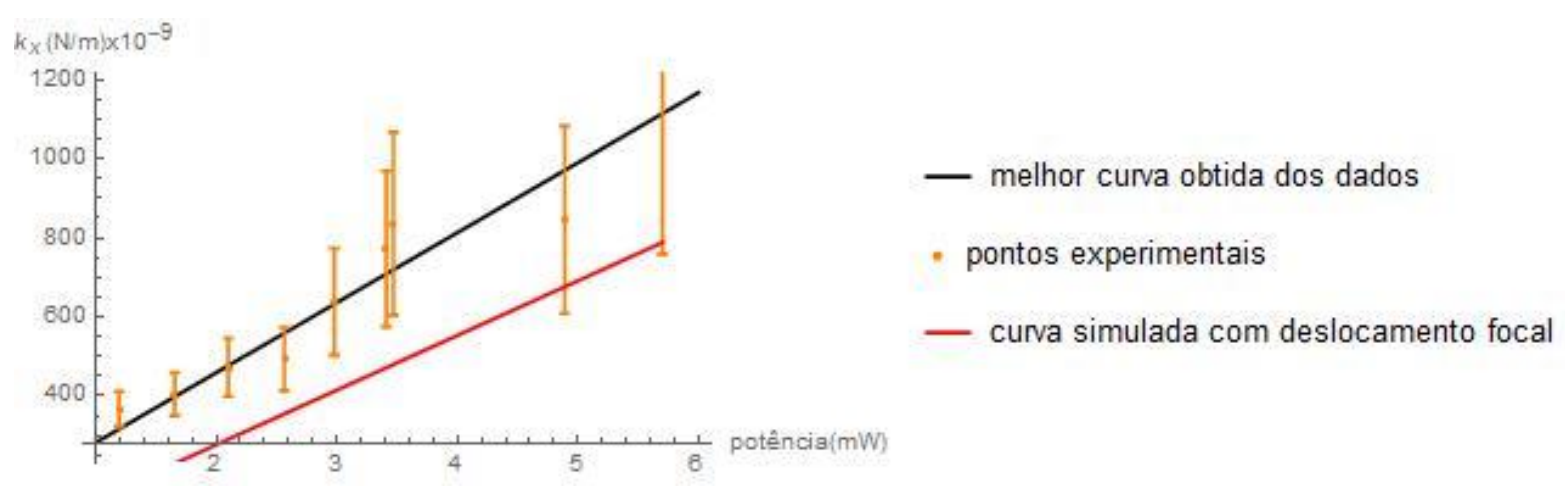

Gráfico 5.6: Os valores de $k_{x}$ obtidos em relação a potência do laser na amostra

Para cada ponto, medimos o tamanho da bead em pixels, e, com as microesferas de diâmetro 2,4 $\mu \mathrm{m}$, obtivemos a posição quadrática média e somamos utilizando a temperatura de $T=294,75 \pm 0,07 K$. Para o erro da posição, foi verificado que a distribuição da posição tem padrão gaussiano (ver Figura 5.11). Desta forma, tomei o desvio padrão como erro e, a partir disso, fiz uma propagação de erros com o erro da temperatura e o desvio padrão. Existem outros erros que não foram possíveis quantificar: primeiro, o erro da medição do tamanho das microesferas; e, segundo, devido ao grande aumento da objetiva, esta não poderia se mover a partir de certo ponto. A distância focal da objetiva era muito pequena e isso impedia o uso de volumes de água maiores que $20 \mathrm{ml}$ no poço onde estavam as esferas. Esse fato impediu a esperada perda de energia cinética resultante da inoculação das microesferas na lamínula, pois se a espera fosse maior que 10 minutos, toda a água secava. Logo, há o componente da energia cinética que não pode ser determinado somada à energia Browniana das microesferas.

Quanto aos resultados das simulações, gostaria primeiramente de salientar que não foi encontrado na literatura nenhum artigo que compare o $k$ obtido experimentalmente e outro simulado a partir Equação 2.132 da forma como está descrito aqui. Por isso, não foi possível comparar estes resultados com o de outros trabalhos. Existem diversos outros fatores, como explicado na Secção 5.1, que interferem na previsão da simulação. Desta maneira, para a simulação sem deslocamento focal, o valor resultante é muito diferente do esperado, representando um centésimo do valor; para o caso com deslocamento focal, dois dos resultados apresentaram valores dentro da margem de erro experimental. 


\subsubsection{Comparado à simulação em relação a y}

Os dados referentes à posição em y estão organizados na Tabela 5.3.

Tabela 5.3: Estão apresentados os valores da potência do laser, sua posição quadrática média vertical, seu $k_{y}$ calculado, o erro dado pelo ajuste na curva, e os valores de $k_{y}$ simulados sem e com o fenômeno do deslocamento focal.

\begin{tabular}{cccccc}
\hline $\begin{array}{c}\text { Potência } \\
(\mathrm{mW})\end{array}$ & $\begin{array}{c}<\Delta \mathrm{y}^{2}>\mathrm{MSD} \\
\mathrm{em} 10^{-14} \mathrm{~m}^{2}\end{array}$ & $k_{y}(N / m) \times 10^{-9}$ & Erro de $k_{y}$ & $\begin{array}{c}k_{y}(N / m) \times 10^{-9} \\
\text { simulado sem } \\
\text { dist. focal }\end{array}$ & $\begin{array}{c}k_{y}(N / m) \times 10^{-9} \\
\text { simulado com } \\
\text { dist. focal }\end{array}$ \\
\hline 0,695 & 1,0557800 & 386 & 49 & 20,612 & 92,7684 \\
1,195 & 1,6511500 & 246 & 20 & 354,408 & 159,508 \\
1,655 & 1,2259300 & 331 & 36 & 490,832 & 220,909 \\
2,100 & 0,5264038 & 775 & 198 & 622,808 & 280,307 \\
2,565 & 0,7975040 & 510 & 86 & 760,716 & 342,375 \\
2,980 & 0,6748280 & 603 & 120 & 883,795 & 397,769 \\
3,470 & 0,4951700 & 821 & 224 & 102,912 & 463,174 \\
3,880 & 0,4174540 & 974 & 315 & 115,071 & 517,901 \\
4,320 & 0,3365900 & 1209 & 485 & 128,121 & 576,632 \\
4,890 & 0,3177070 & 1280 & 544 & 145,025 & 652,716 \\
\hline
\end{tabular}


Para os pontos obtidos, foi traçado o gráfico apresentado abaixo.

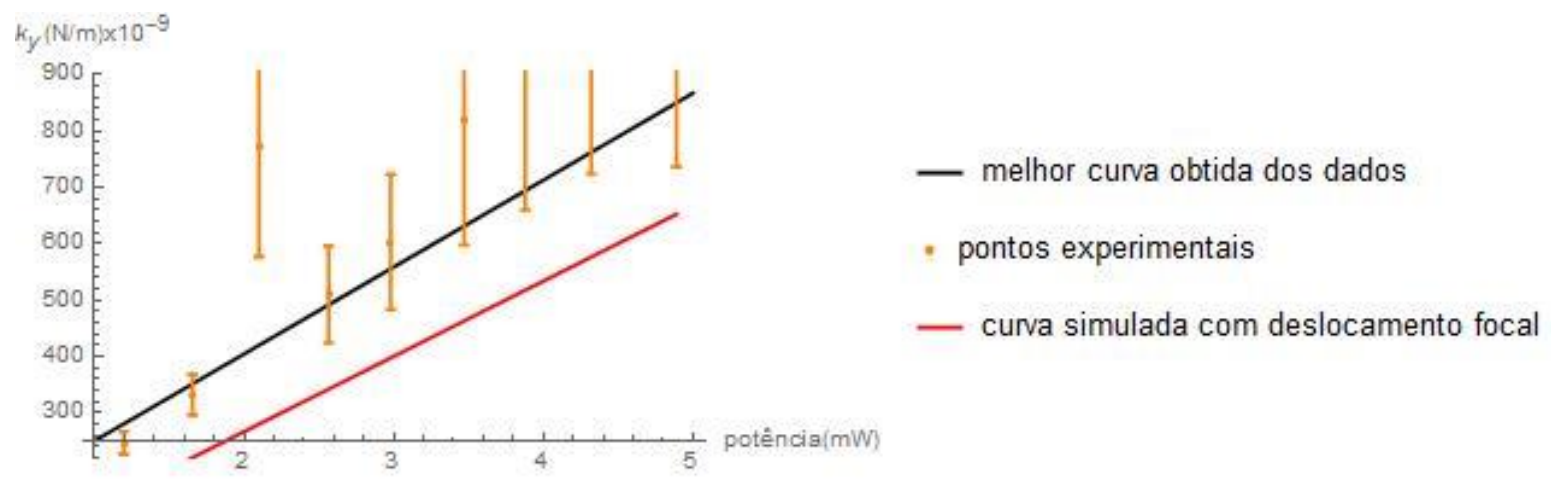

Gráfico 5.7: Os valores de $k_{y}$ obtidos em relação à potência do laser na amostra

Neste caso, os pontos experimentais não foram compatíveis com nenhum ponto simulado.

5.3.3 Com método de análise gaussiano comparado à simulação em relação ao eixo $x$

Agora, serão apresentados os resultados utilizando o mesmo conjunto de dados e o método do ajuste da curva Gaussiana, explicado na Secção 4.3.3. Esses dados estão apresentados na Tabela 5.4. 
Tabela 5.4: Estão apresentados a potência do laser, os valores experimentais de $k_{x}$, o erro experimental e os valores da simulação sem e com deslocamento focal.

\begin{tabular}{ccccc}
\hline $\begin{array}{c}\text { Potência } \\
(\mathrm{mW})\end{array}$ & $k_{x}(N / m) \times 10^{-9}$ & Erro de $k_{x}$ & $\begin{array}{c}k_{x}(N / m) \times 10^{-9} \\
\text { simulado sem desl. } \\
\text { focal }\end{array}$ & $\begin{array}{c}k_{x}(N / m) \times 10^{-9} \\
\text { simulado com desl. } \\
\text { Focal }\end{array}$ \\
\hline 0,695 & 193 & 16 & 12,8417 & 96,0885 \\
1,195 & 353 & 44 & 22,0803 & 165,2170 \\
1,655 & 372 & 54 & 30,5799 & 228,8150 \\
2,100 & 465 & 73 & 38,8023 & 290,3390 \\
2,565 & 475 & 80 & 47,3942 & 354,6290 \\
2,980 & 560 & 135 & 55,0623 & 412,0050 \\
3,410 & 847 & 197 & 63,0075 & 471,4560 \\
3,470 & 879 & 231 & 64,1161 & 479,7510 \\
4,890 & 910 & 237 & 90,3539 & 676,0760 \\
5,700 & 1230 & 732 & 105,3200 & 788,0640 \\
\hline
\end{tabular}

Para os pontos obtidos foi traçado o gráfico apresentado abaixo.

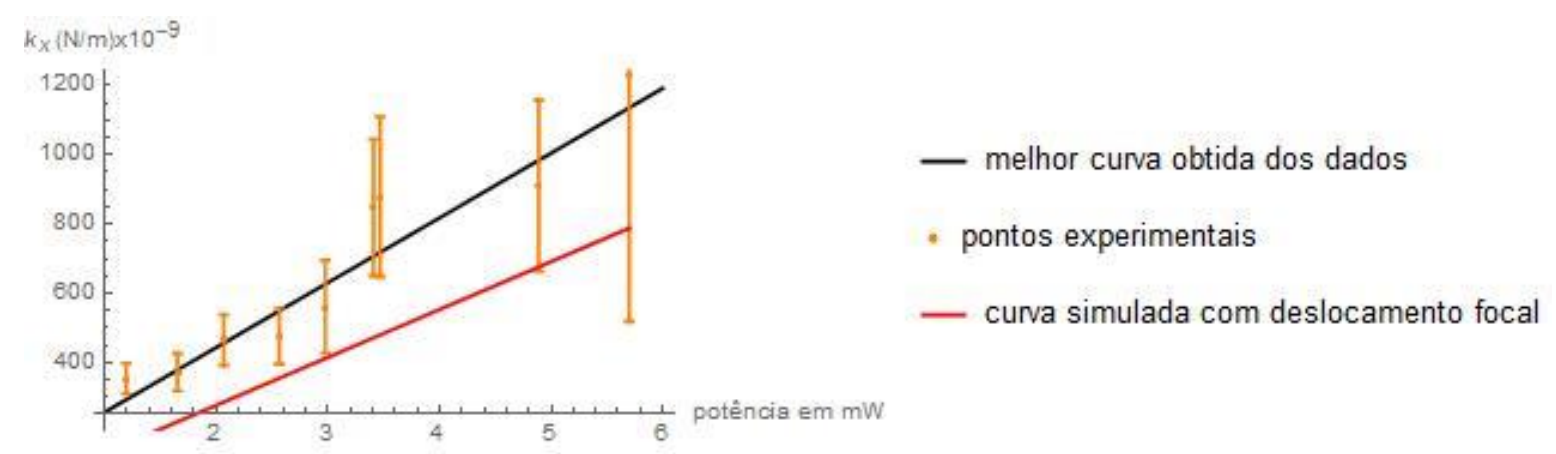

Gráfico 5.8: Os valores de $k_{x}$ obtidos em relação a potência do laser na amostra. 
5.3.4 Com método de análise Gaussiano comparado à simulação em relação ao eixo y

Tomando o conjunto de posições em relação eixo y do ajuste da curva Gaussiana temos os dados apresentados na Tabela 5.5.

Tabela 5.5: Estão apresentados o potencial do laser, os valores experimentais de $k_{y}$, o erro experimental, e os valores da simulação sem e com deslocamento focal.

\begin{tabular}{ccccc}
\hline $\begin{array}{c}\text { Potência } \\
(\mathrm{mW})\end{array}$ & $k_{y}(N / m) \times 10^{-9}$ & Erro de $k_{y}$ & $\begin{array}{c}k_{y}(n / m) \times 10^{-9} \\
\text { simulado sem desl. } \\
\text { focal }\end{array}$ & $\begin{array}{c}k_{y}(n / m) \times 10^{-9} \\
\text { simulado com } \\
\text { desl. focal }\end{array}$ \\
\hline 0,695 & 135 & 20 & 20,612 & 927,684 \\
1,195 & 249 & 20 & 354,408 & 159,508 \\
1,655 & 280 & 36 & 490,832 & 220,909 \\
2,100 & 507 & 86 & 622,808 & 280,307 \\
2,565 & 631 & 86 & 760,716 & 342,375 \\
2,980 & 710 & 120 & 883,795 & 397,769 \\
3,470 & 777 & 224 & 102,912 & 463,174 \\
3,880 & 812 & 315 & 115,071 & 517,901 \\
4,320 & 986 & 485 & 128,121 & 576,632 \\
4,890 & 1395 & 544 & 145,025 & 652,716 \\
\hline
\end{tabular}

Para os pontos obtidos foi traçado o gráfico apresentado abaixo.

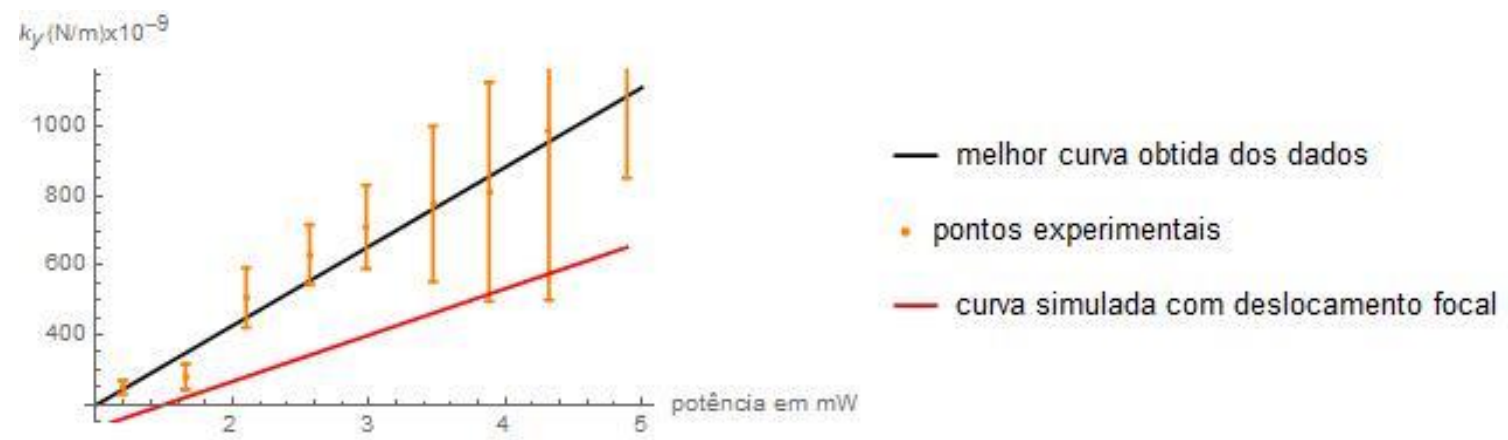

Gráfico 5.9: Os valores de $k_{y}$ obtidos para o ajuste gaussiano. 
No gráfico acima, é possível observar que a curva simulada se ajusta a alguns dos dados experimentais, ou seja, esse método de análise parece estar um pouco mais preciso. Para maior precisão, seria necessária uma maior coleta de dados, sendo que, cada ponto tem em média 1000 pontos coletados de posição das microesferas.

\section{p probabilidade de Marxell}

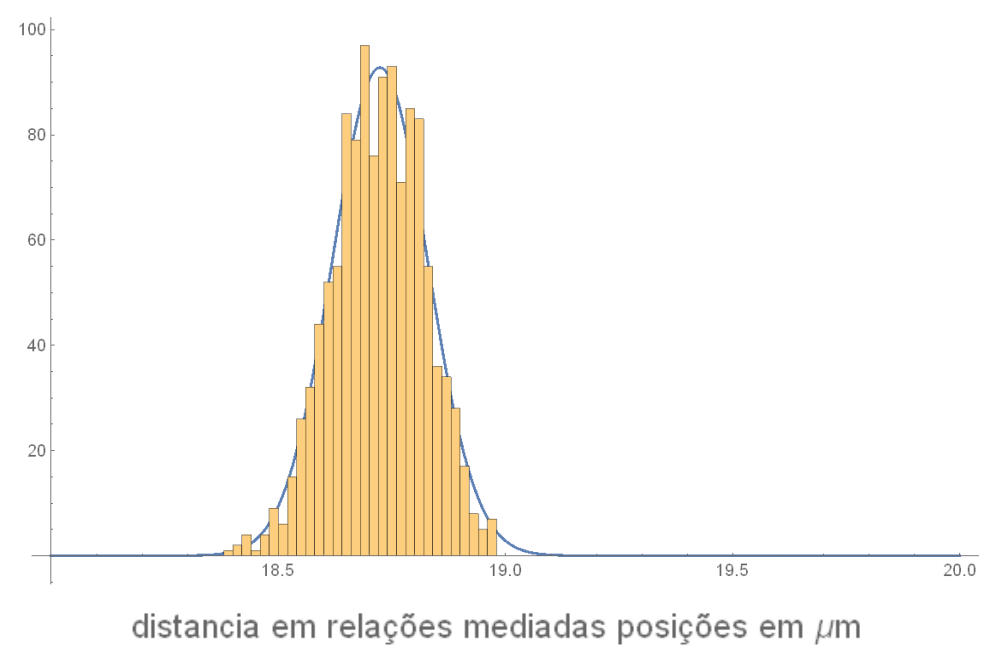

Gráfico 5.10: Ajuste gaussiano dos pontos obtidos das posições das microesferas mostrado pelo histograma que apresenta perfil gaussiano.

\subsection{Resultados experimentais do experimento de força viscosa comparada com as simulações} do $\boldsymbol{k}_{\boldsymbol{x} m}$

Os dados obtidos podem ser observados na Tabela 5.6, onde $k_{x m}$ é denotado assim diferencia do outro $k_{x}$, obtido pelo método Gaussiano. 
Tabela 5.6: Estão apresentados os valores da potência do laser, do deslocamento calculado pelas imagens $\left\langle\Delta \mathrm{x}>\right.$, o valor de $k_{x m}$ experimental, o erro do ajuste da curva e os valores simulados.

\begin{tabular}{cccccc}
\hline $\begin{array}{c}\text { Potência } \\
(\mathrm{mW})\end{array}$ & $<\Delta \mathrm{x}>\mathrm{x} 10^{-6}(\mathrm{~m})$ & $k_{x m}(N / m) \times 10^{-}$ & $\begin{array}{c}\text { Erro de } \\
k_{x m}\end{array}$ & $\begin{array}{c}k_{x m}(N / m) \times 10^{-9} \\
\text { simulado sem } \\
\text { desl. focal }\end{array}$ & $\begin{array}{c}k_{x m}(N / m) \times 10^{-9} \\
\text { simulado com } \\
\text { desl.focal }\end{array}$ \\
\hline 7,20 & 0,6119 & 448 & 12 & 104,551 & 593,352 \\
7,98 & 0,5980 & 458 & 13 & 115,877 & 657,632 \\
9,55 & 0,5470 & 501 & 80 & 138,675 & 787,016 \\
10,70 & 0,3630 & 755 & 80 & 155,374 & 881,787 \\
11,86 & 0,3500 & 783 & 100 & 172,218 & 977,383 \\
13,03 & 0,3300 & 831 & 100 & 189,207 & 1073,800 \\
14,22 & 0,2200 & 1246 & 100 & 206,487 & 1171,870 \\
15,33 & 0,1870 & 1466 & 100 & 222,606 & 1263,350 \\
16,77 & 0,1251 & 2193 & 100 & 243,516 & 1382,020 \\
\hline
\end{tabular}

Para os pontos obtidos foi traçado o gráfico apresentado abaixo.

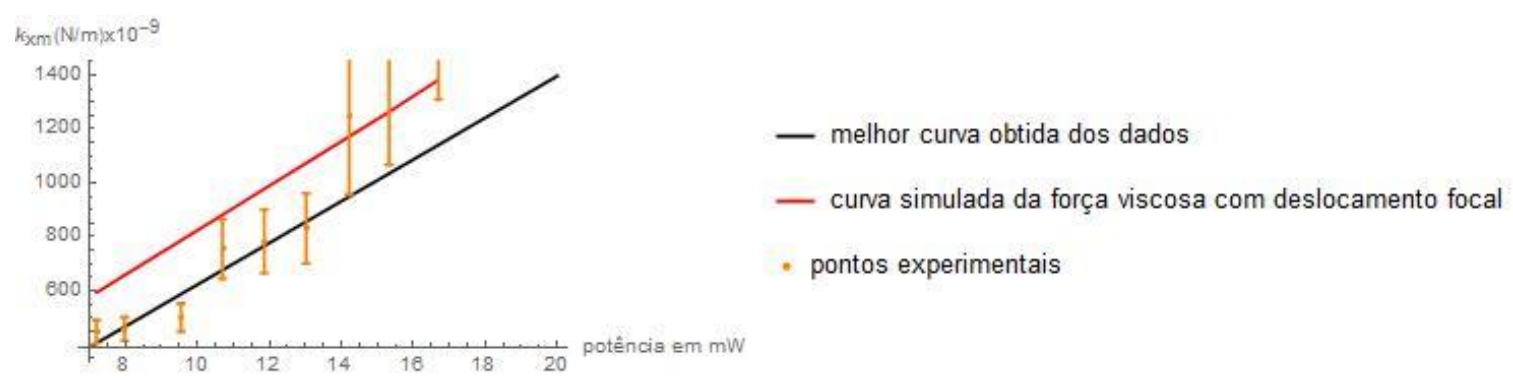

Gráfico 5.11: Curva ajustada para os resultados da força viscosa.

Nessa simulação, observamos que os valores dos pontos experimentais estão menores para maiores potências se comparados ao experimento gaussiano. Sugere-se que esse resultado é decorrente da vibração causada pelo motor de passo, o que grava um movimento na bead indesejado, alterando a força resultante em x. Em decorrência desse problema, o valor de $k_{x}$ tornou-se menor. O teste foi realizado na simulação onde subtraíram-se as forças 
e o resultado parece ser plausível, visto que havia 4 pontos na simulação, e os mesmos encontram-se dentro da barra de erro.

Para o erro desse experimento, tomou-se o erro da posição, o qual correspondia a 0,5 pixel da medida obtida no "Mathematica", mais o erro da velocidade. Foi utilizada propagação de erro para o cálculo final. O programa utilizado para simular os valores de $k_{x m}$ consta no Apêndice E. Neste programa, é usado o mesmo modelo do $k$ axial do programa mostrado no Apêndice C.

5.5 Resultado das simulações para $k_{z}$ ou $k_{\text {axial }}$ com e sem distância focal, com o deslocamento focal igual ao caso experimental radial

Mesmo não sendo possível fazer uma calibração para a direção axial, realizamos o cálculo com a força simulada. Os resultados obtidos para o deslocamento focal estão mostrados na Tabela 5.7 e no Gráfico 5.12. Na Tabela 5.8 e no Gráfico 5.13, temos os resultados sem o deslocamento focal, sendo que, para as Tabelas 5.9 e 5.10, temos as comparações dos valores simulados de $k_{y}$. Na primeira tabela, estão os resultados para $\rho_{0}=0$ e, para as Tabelas 5.9 e 5.10 , foram simulados utilizando $\rho_{0}=0.3$, o qual é o mesmo da simulação de $k_{y}$ e $k_{x}$. 
Tabela 5.7: Estão apresentados os valores de potência, em $\mathrm{mW}$, e os valores para $k_{z}$ simulados, em $10^{-6}(\mathrm{~N} / \mathrm{m})$, levando em conta o deslocamento focal.

\begin{tabular}{|c|c|}
\hline Potência (mW) & $k_{z}(N / m) \times 10^{-6}$ \\
\hline 0,695 & 112,850 \\
\hline 1,195 & 215,768 \\
\hline 1,655 & 298,826 \\
\hline 2,070 & 379,174 \\
\hline 2,565 & 463,135 \\
\hline 2,980 & 538,067 \\
\hline 3,470 & 615,707 \\
\hline 3,880 & 626,541 \\
\hline 4,320 & 700,570 \\
\hline 4,890 & 780,016 \\
\hline 7,200 & 1300,030 \\
\hline 7,980 & 1440,860 \\
\hline 9,550 & 1724,340 \\
\hline 10,700 & 1931,980 \\
\hline 11,860 & 2141,430 \\
\hline 13,030 & 2352,690 \\
\hline 14,220 & 2567,550 \\
\hline 15,330 & 2767,970 \\
\hline 16,770 & 3015,340 \\
\hline
\end{tabular}




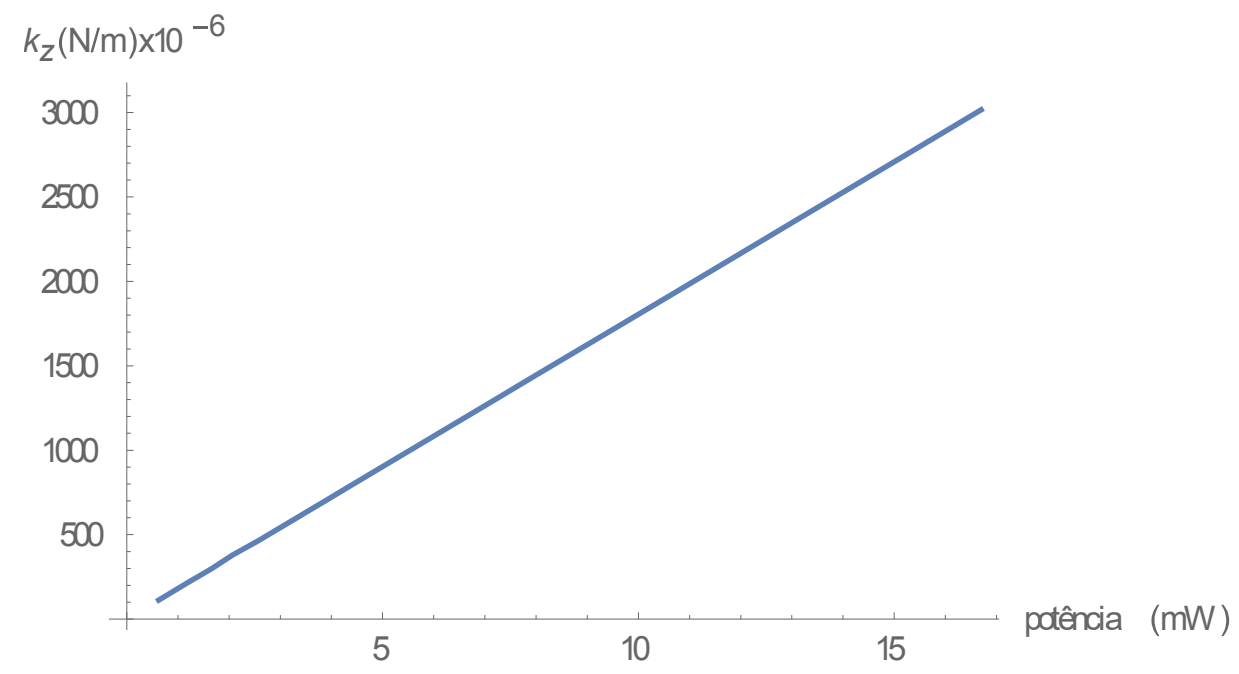

Gráfico 5.12: Gráfico da potência versus $k_{z}$ com deslocamento focal. 
Tabela 5.8: Estão apresentados os valores de potência, em $\mathrm{mW}$, e os valores para $k_{z}$ simulados, em $10^{-9}(\mathrm{~N} / \mathrm{m})$, sem levar em conta o deslocamento focal.

\begin{tabular}{|c|c|}
\hline Potência (mW) & $k_{z}(N / m) \times 10^{-9}$ \\
\hline 0,695 & 54,4035 \\
\hline 1,195 & 104,020 \\
\hline 1,655 & 144,061 \\
\hline 2,070 & 182,796 \\
\hline 2,565 & 223,272 \\
\hline 2,980 & 259,396 \\
\hline 3,470 & 296,826 \\
\hline 3,880 & 302,048 \\
\hline 4,320 & 337,737 \\
\hline 4,890 & 376,037 \\
\hline 7,200 & 626,729 \\
\hline 7,980 & 694,624 \\
\hline 9,550 & 831,286 \\
\hline 10,700 & 931,388 \\
\hline 11,860 & 1032,360 \\
\hline 13,030 & 1134,200 \\
\hline 14,220 & 1237,790 \\
\hline 15,330 & 1334,410 \\
\hline 16,770 & 1453,660 \\
\hline
\end{tabular}




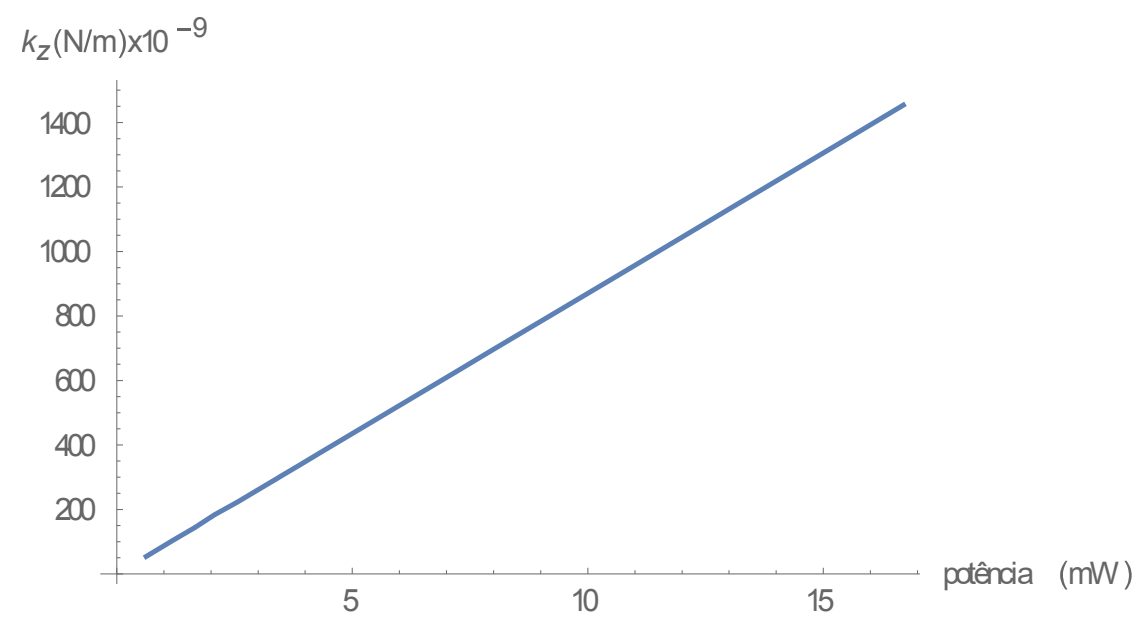

Figura 5.13: Gráfico da simulação do $k_{z}$ sem o deslocamento focal versus a potência.

Por fim, temos a comparação entre o $k_{z}$ e radial para $\rho_{0}=0,3$, como constam na Tabela 5.9.

Tabela 5.9: Estão apresentados os valores da potência do laser e a comparação do $k_{y}$ radial com com $k_{z}$, sem deslocamento focal.

\begin{tabular}{ccc}
\hline Potência $(\mathrm{mW})$ & $\begin{array}{c}k_{\text {radial }}(N / m) \times 10^{-9} \mathrm{sem} \\
\text { deslocamento focal }\end{array}$ & $\begin{array}{c}k_{\text {axial }}(N / m) \times 10^{-9} \mathrm{sem} \\
\text { deslocamento focal }\end{array}$ \\
\hline 0,695 & 20,612 & 314,740 \\
1,195 & 354,408 & 541,172 \\
1,655 & 490,832 & 749,489 \\
2,100 & 622,808 & 951,013 \\
2,565 & 760,716 & 116,159 \\
2,980 & 883,795 & 134,953 \\
3,470 & 102,912 & 157,144 \\
3,880 & 115,071 & 175,711 \\
4,320 & 128,121 & 195,637 \\
4,890 & 145,025 & 221,450 \\
\hline
\end{tabular}


Tabela 5.10: Estão apresentados os valores da potência do laser e a comparação do $k_{y}$ radial com $k_{\text {axial }}$, considerando o deslocamento focal.

\begin{tabular}{ccc}
\hline Potência $(\mathrm{mW})$ & $\begin{array}{c}k_{\text {radial }}(N / m) \times 10^{-9} \mathrm{com} \\
\text { deslocamento focal }\end{array}$ & $\begin{array}{c}k_{\text {axial }}(N / m) \times 10^{-6} \mathrm{com} \\
\text { deslocamneto focal }\end{array}$ \\
\hline 0,695 & 92,7684 & 86,3671 \\
1,195 & 159,5080 & 148,5020 \\
1,655 & 220,9090 & 205,6660 \\
2,100 & 280,3070 & 260,9650 \\
2,565 & 342,3750 & 318,7510 \\
2,980 & 397,7690 & 370,3220 \\
3,470 & 463,1740 & 431,2140 \\
3,880 & 517,9010 & 482,1650 \\
4,320 & 576,6320 & 536,8400 \\
4,890 & 652,7160 & 607,6770 \\
\hline
\end{tabular}

Sabendo que, por outras referências ${ }^{[4]}$, os valores radial e axial da constante estão na mesma magnitude. Para o caso considerando o deslocamento focal, o programa apresentou problemas que ainda não foram identificados, isso fez com que os resultados apresentassem valores com dferenças de até 1000 vezes.

\subsection{Discussão sobre as dificuldades de simular as forças ópticas no regime de Mie}

As Equações 2.131 e 2.132 são muito oscilatórias, além de apresentarem singularidades e funções consideradas difíceis de serem calculadas computacionalmente. Um exemplo dessa dificuldade pode ser dado quando elas apresentam uma grande oscilação. Para esse caso, calcular essas integrais, as quais podem apresentar grande diferença entre o denominador e o numerador, como na função de Bessel presente nelas, pode resultar em overflow e underflow. Isso aconteceu no cálculo realizado, onde o valor da integral variou entre $10^{-148}$ à $10^{148}$, ou seja, números que o "Mathematica" considera zero e infinito. Para contornar esse problema, multiplicamos a integral 
pelo fator $\frac{(n-m) !}{(n+m) !}$, com intuito de diminuir essa oscilação ${ }^{[1][9]}$ e obtermos um resultado satisfatório. Podemos observar nos gráficos 3D construídos para a integral com deslocamento focal, a variação de valores que a função sem ser integrada apresenta.

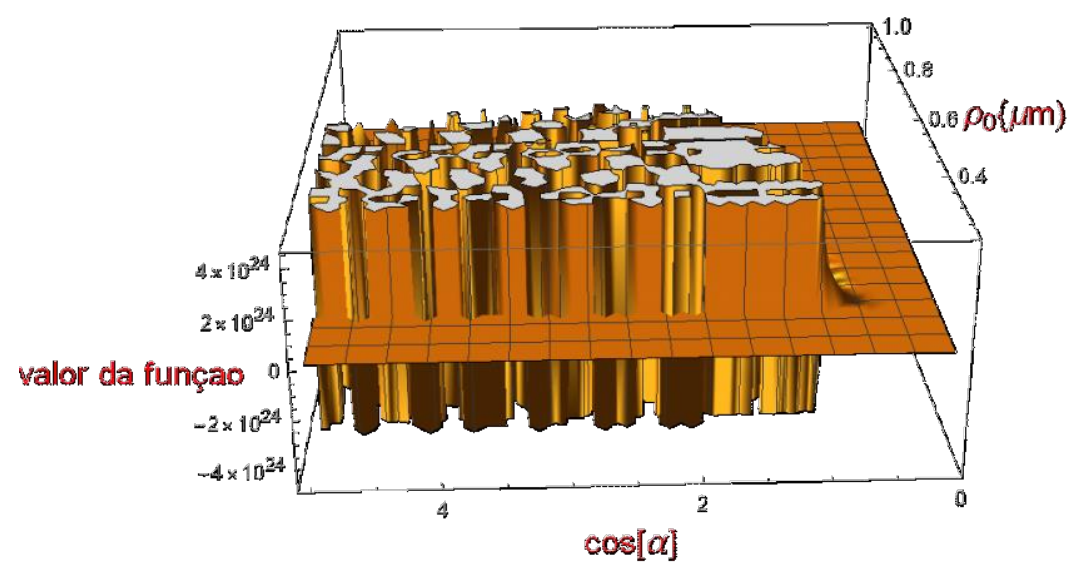

Gráfico 5.14: Valor da integral I1 com deslocamento focal para $n=1, m=0$, variando $\mathrm{x}$ e $\rho_{0}$

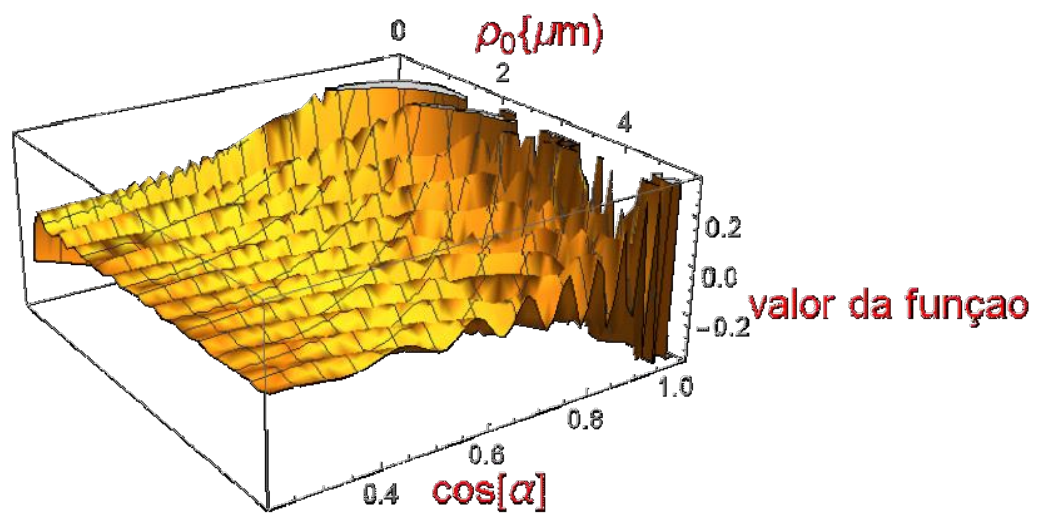

Gráfico 5.15: Valor da integral I1 com deslocamento focal para $n=22, m=22$, variando $x$ e $\rho_{0}$. 


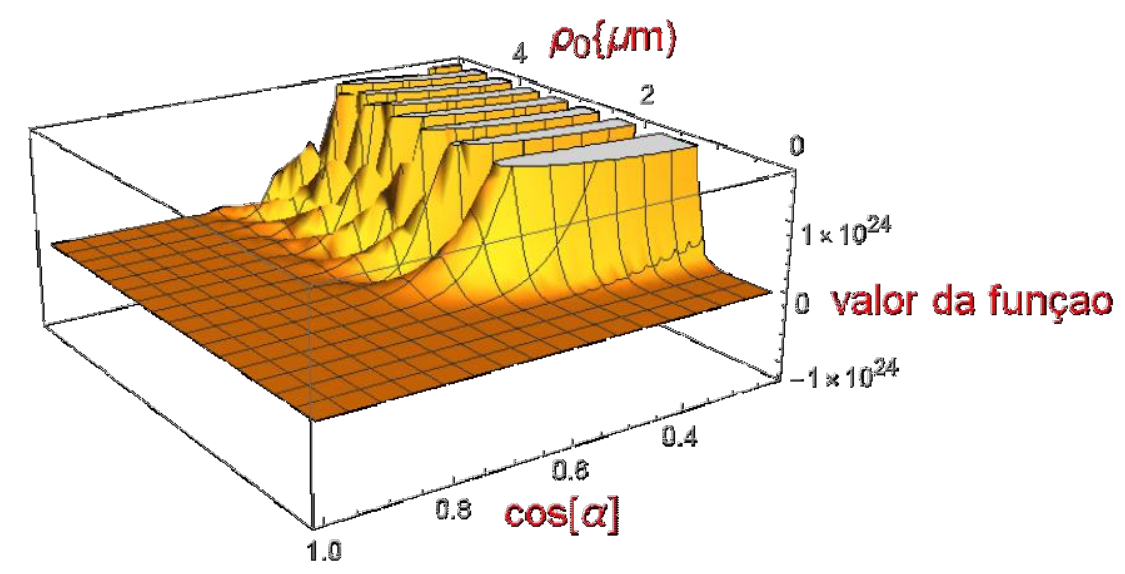

Gráfico 5.16: Valor da integral I1, sem deslocamento focal, para $n=1, m=0$, variando x e $\rho_{0}$.

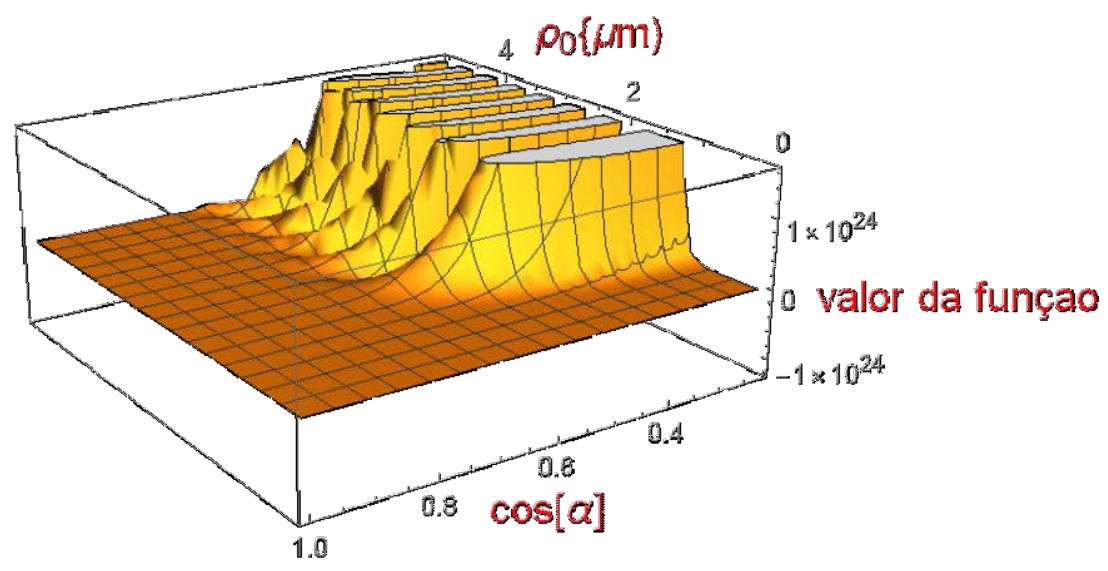

Gráfico 5.17: Valor da integral I1 sem deslocamento focal para $n=22, m=22$,variando x e $\rho_{0}$.

Outro problema é que, como a integral varia em valores, é necessário fazer várias recursões na integral, o que leva à somatória aritmética e conversão de vários números de ponto flutuante. Caso esse número seja pequeno, o erro ou rando-oof do número de ponto flutuante cresceria muito rápido devido ao overflwor e da recursão, fazendo com que ultrapasse o erro absoluto para determinada precisão. Logo, é necessário modificar a precisão que o programa faz as operações como suas recursões, além de que, são necessários vários algoritmos para lidar com funções altamente oscilatórias, como método do trapézio ou de adaptação global. No entanto, não houve tempo para dedicar-me a complexidade do assunto, desta forma, foram usadas duas soluções de métodos do "Mathematica", a primeira é oscilatory seletion que analisa a integral e determina qual é o melhor algarismo a ser usado. A 
segunda solução foi o uso de equações de Bessel, utilizando o algoritmo Bessel Infinite Range Method. 


\section{Referências}

[1] A. A. R. Neves. Força óptica em pinças ópticas: estudo teórico e experimental. 2006. 169 p. Tese (doutorado) - Universidade Estadual de Campinas, Instituto de Física GlebWataghin, Campinas, SP.

[2] M. Jahnel, M.Behrndt, A.Jannasch, E.Schäffer, and S. W. Grill, "Measuring the complete force field of an optical trap,"Optics Letters, vol. 36, no. 7, pp. 1260,2011.

[3] A. Rohrbach, H. Kress, and E. H. K.Stelzer, "Reply to comment on "Trapping force, force constant, and potential depths for dielectric spheres in the presence of spherical aberrations," Applied Optics, vol. 43, no. 9, pp. 1827-1829,2004.

[4] V. Bormuth, A.Jannasch, M. Ander, C. M. van Kats, A. van Blaaderen, J. Howard, and E.Schäffer, "Optical trapping of coated microspheres,"Optics Express, vol. 16, no. 18, pp. 13831,2008 .

[5]Fontes, A. Microanálise com Pinças Ópticas, 2004, 271 p. Tese (doutorado)-Universidade Estadual de Campinas, Instituto de Física GlebWataghin, Campinas, SP.

[6]A. A. R. Neves, A.Fontes, C. L. Cesar, A. Camposeo, R.C ingolani, and D. Pisignano, "Axial optical trapping efficiency through a dielectric interface,"Physical Review E Statistical, Nonlinear, and Soft Matter Physics, vol. 76, no. 6, pp. 1-8,2007.

[7] F. Merenda, G. Boer, J. Rohner, G. Delacrétaz, and R. P.Salathé, "Escape trajectories of single-beam optically trapped micro-particles in a transverse fluid flow,"Optics Express, vol. 14, no. 4, pp. 1685, 2006.

[8] G. Volpe, and G. Volpe, "Simulation of a Brownian particle in an optical trap,"American Journal of Physics, vol. 81, no. 3, pp. 224-230, 2013.

[9] T. Collection, T, “Advanced Numerical Integration in MATHEMATICA,"Mathematica, vol. 179, no. 1, pp. 33-38, 2008.

[10] A. G. Hendricks, and Y. E. Goldman, "Calibration of Optical Tweezers,"Society, vol. 1486, no. 1, pp. 537-552,2004.

[11]W. N. S. Wan Aziz, S. K. Ayop, M. Y. Hamid, and Y. Munajat, "Simple Determination of the Stiffness of an Optical Trap Using Video Microscopy and Particle Tracking,"Buletin Optik, vol. 2, pp. 1-6, 2016. 


\section{Capítulo 6}

\section{Conclusão e Perspectivas}

Neste trabalho, foi proposta a construção de um arranjo simplificado de pinça óptica e sua completa caracterização. Foi estudada também a teoria de seu funcionamento através de simulações que atingiram a ordem de grandeza dos resultados experimentais, mas esses resultados não estão isentos de erro por falta de inclusão de outros fenômenos e de um estudo mais aprofundado sobre integração em funções altamente oscilatórias. Desta forma, as simulações ainda não são perfeitas e necessitam de maiores desenvolvimentos. Quanto à parte experimental, desenvolvemos um arranjo de armadilha óptica controlado por um sistema eletrônico, sendo este devidamente calibrado, estando em funcionamento.

Como continuidade, propomos a adaptação desse arranjo a um sistema de Microscopia de Força e Tração, já em uso no LabMํㄹ de Microrreologia do Instituto de Física da USP. Vimos que é preciso refinar o método experimental, com a proposta de aquisição de um novo microscópio onde seja possível mover a amostra livremente em z, bem como o uso de um motor piezoelétrico e de um motor de passo com velocidade variável bem determinada. Além da possibilidade de mover a amostra nos eixos $\mathrm{x}, \mathrm{y}, \mathrm{z}$, se faz necessário um software melhor para detecção das mudanças de posição de deslocamento das microesferas. Desta forma, com essas melhorias, teríamos bastante confiança no valor experimental da calibração, e, conseqüentemente, poderíamos melhorar a simulação com tais resultados. Mesmo com as limitações experimentais, o sistema funciona e abre possibilidades para diversas aplicações na área de caracterização de propriedades reológicas de sistemas fluídicos. 


\section{Apêndice A}

Para determinar os coeficientes da onda espalhada e interna, primeiro vamos definir a equação de Henkel a partir da sua definição (Equação 2.105). No Capítulo 2 essa equação só foi definida em sua forma assintóptica, porém, nos limites da esfera ela não se apresenta em sua forma assinóptica, mas sim em sua forma esférica.

$$
y_{n}(k r)=\frac{(-1)^{n+1}}{2^{n}(k r)^{n+1}} \sum_{s=0}^{\infty} \frac{(-1)^{s}(s-n) !}{s !(2 s-2 n) !}(k r)^{2 s}
$$

Onde $y_{n}(k r)$ erepresenta a função de Bessel de segunda ordem esférica logo, pela definição 2.105, temos:

$$
h_{n}^{1}=2^{n}(k r)^{n} \sum_{s=0}^{\infty} \frac{(-1)^{s}(s+n) !}{s !(2 s+2 n+1) !}(k r)^{2 s}+i \frac{(-1)^{n+1}}{2^{n}(k r)^{n+1}} \sum_{s=0}^{\infty} \frac{(-1)^{s}(s-n) !}{s !(2 s-2 n) !}(k r)^{2 s}
$$

Usando essas equações é possível definiras equações de Riccati-Bessel, que serão úteis para definir os coeficientes de espalhamento e do interior da esfera que são:

$$
\psi_{n}=\rho j_{n}(\rho) \quad \xi_{n}(\rho)=\rho h_{n}^{(1)}(\rho)
$$

onde, a equação de Maxwell requer que os pontos sejam contínuos entre as regiões internas e externas, e que as ondas estejam espalhadas no exterior inteiro da esfera para que $\varepsilon e \mu$ sejam contínuas, desta forma, temos a condição que geram as novas variáveis.

$$
x=k_{1} d=\frac{2 \pi d n_{2}}{\lambda} \quad \text { e } \quad M x=\frac{n_{1}}{n_{2}} k_{1} d=k_{2} d
$$

Note que o fator de tamanho $x$ e o índice relativo $M$ de refração são independentes matematicamente, mas não fisicamente já que ambos variam o comprimento de onda.

Logo, definimos as condições de contorno na fronteira da esfera, onde $\varepsilon e \mu$ sejam contínuos, porém, há uma descontinuidade em relação ao meio da partícula. Então, introduzimos a condição de que:

$$
\begin{aligned}
& {\left[\vec{E}_{2}(\vec{x})-\vec{E}_{1}(\vec{x})\right] \times \hat{r}=0} \\
& {\left[\vec{H}_{2}(\vec{x})-\vec{H}_{1}(\vec{x})\right] \times \hat{r}=0}
\end{aligned}
$$

onde os campos magnéticos e elétricos forma definidos no Capítulo 2 nas Equações 2.84 e 2.85 .

Para os modos TE do campo elétrico, nas equações temos que campos elétricos têm uma variável $z_{n}(k r) X_{n m}(\theta, \phi)$ onde $z_{n}(k r)$ e é a função de Bessel esférica ou de Henkel. 
Logo, as condições de contorno são:

$$
\begin{gathered}
\left.\left(\vec{E}_{1}(\vec{x})-\vec{E}_{2}(\vec{x})\right)\right)^{T E} \times \hat{r}=\left(\vec{E}_{l}^{T E}(x)+\vec{E}_{e}^{T E}(x)-\vec{E}_{\text {int }}^{T E}(x)\right) \times \hat{r}= \\
E_{0} \sum_{n, n}\left(b_{n m} h_{n}^{1}(x)+G_{n m}^{T E} j_{n}(x)+d_{n m} j_{n}(M x)\right) \vec{X}_{n m}(\theta, \phi) \times \hat{r}=0
\end{gathered}
$$

Que deve ser válida para todos os ângulos.

$$
\left.b_{n m} h_{n}^{1}(x)+G_{n m}^{T E} j_{n}(x)=d_{n m} j_{n}(M x)\right)
$$

Já para os modos TE dos campos magnético temos $\hat{r} \times\left(\nabla \times z_{n}(k r) \vec{X}_{n m}(\theta, \phi)\right)$.Onde, utilizando o fato que para coordenadas esféricas o vetor gradiente é dado por:

$$
\vec{\nabla}=\hat{r} \frac{\partial}{\partial r}+\hat{\theta} \frac{1}{r} \frac{\partial}{\partial \theta}+\hat{\phi} \frac{1}{r \operatorname{sen} \theta} \frac{\partial}{\partial \phi}
$$

e as relações:

$$
\nabla \times \vec{L}=i\left[2 \nabla-\vec{r} \nabla^{2}+(r \cdot \nabla) \nabla\right]
$$

Considerando para equação de Helmozt, temos que:

$$
\left[\frac{1}{r^{2}} \frac{\partial}{\partial r}\left(r^{2} \frac{\partial}{\partial r}\right)-\frac{L^{2}}{r^{2}}+k\right] \psi=0
$$

onde $\psi$ é a equação que satisfaz as equações de Maxwell, ou seja, utilizando as equações do campo elétrico magnético e, sendo k uma constante qualquer, temos que, reescrevendo essas equações:

$$
\begin{aligned}
& r \frac{\partial}{\partial r}\left(\nabla Y_{n m}\right)=r \frac{\partial}{\partial r}\left[\hat{r} \frac{\partial Y_{n m}}{\partial r}+\hat{\theta} \frac{1}{r} \frac{\partial Y_{n m}}{\partial \theta}+\hat{\phi} \frac{1}{r \operatorname{sen} \theta} \frac{\partial Y_{n m}}{\partial \phi}\right]=\frac{-1}{r}\left[\hat{\theta} \frac{\partial Y_{n m}}{\partial \theta}+\right. \\
& \left.\hat{\phi} \frac{1}{\operatorname{sen} \theta} \frac{\partial Y_{n m}}{\partial \phi}\right]=-\nabla Y_{n m}
\end{aligned}
$$

onde, para toda equação $f(r)$, seja ela uma função de Bessel ou não, obedece à seguinte propriedade: 


$$
\begin{gathered}
\left(\nabla \times f(r) \vec{X}_{n m}(\theta, \phi)\right)=\nabla f(r) \times \vec{X}_{n m}+f(r) \nabla \times \vec{X}_{n m}=\frac{\partial f(r)}{\partial r} \hat{r} \times \vec{X}_{n m}+ \\
\frac{f(r)}{\sqrt{n(n+1)}} \nabla \times \vec{L} Y_{n m}=\frac{\partial f(r)}{\partial r} \hat{r} \times \vec{X}_{n m}+\frac{i f(r)}{\sqrt{n(n+1)}}\left[\left[2+r \frac{\partial}{\partial r}\right] \nabla+\vec{r} \frac{n(n+1)}{r^{2}}\right] Y_{n m}=\frac{\partial f(r)}{\partial r} \hat{r} \times \\
\vec{X}_{n m}+\frac{i f(r)}{\sqrt{n(n+1)}}\left[\nabla Y_{n m}+\vec{r} \frac{n(n+1)}{r^{2}} Y_{n m}\right]=\frac{\partial f(r)}{\partial r} \hat{r} \times \vec{X}_{n m}+\frac{i f(r)}{\sqrt{n(n+1)}}\left[-i \frac{\vec{r}}{r^{2}} \times \vec{L} Y_{n m}+\right. \\
\left.\vec{r} \frac{n(n+1)}{r^{2}} Y_{n m}\right]=\frac{\partial f(r)}{\partial r} \hat{r} \times \vec{X}_{n m}+f(r) \frac{\vec{r}}{r^{2}} \times \vec{X}_{n m}+i \sqrt{n(n+1)} \frac{f(r)}{r^{2}} Y_{n m} \vec{r}=\frac{1}{r} \frac{\partial(r f(r))}{\partial r} \hat{r} \times \\
\vec{X}_{n m}+i \sqrt{n(n+1)} \frac{f(r)}{r^{2}} Y_{n m} \vec{r}
\end{gathered}
$$

Como o segundo termo do produto vetorial é nulo, temos que:

$$
\left(\nabla \times f(r) \vec{X}_{n m}(\theta, \phi)=-\frac{1}{r} \frac{\partial(r f(r))}{\partial r} \vec{X}_{n m}\right.
$$

Logo,

$$
\begin{array}{r}
\left(\vec{H}_{1}(\vec{x})-\vec{H}_{2}(\vec{x})\right)^{T E} \times \hat{r}=\left(\vec{H}_{l}^{T E}(x)+\vec{H}_{e}^{T E}(x)-\vec{H}_{i n t}^{T E}(x)\right) \times \hat{r}= \\
E_{0} \sum_{n, n}\left(b_{n m} \frac{\left[x h_{n}^{1}(x)\right]^{\prime}}{Z_{1} x}+G_{n m}^{T E} \frac{\left[x j_{n}(x)\right]^{\prime}}{Z_{1} x}+d_{n m} \frac{\left[M x j_{n}[M x]\right.}{Z_{2} M x}\right) \vec{X}_{n m}(\theta, \phi) \times \hat{r}=0
\end{array}
$$

Logo,

$$
b_{n m}\left[x h_{n}^{1}(x)\right]^{\prime}+G_{n m}^{T E}\left[x j_{n}(x)\right]^{\prime}=\frac{z_{1} k_{1}}{Z_{2} k_{2}} d_{n m}\left[M x j_{n}[M x]\right]^{\prime}=\frac{\mu_{1}}{\mu_{2}} d_{n m}\left[M x j_{n}[M x]\right]^{\prime}
$$

Onde os apóstrofos indicam a diferença entre argumento dentro do parêntese. Assim, usado as equações de Riccati-Bessel e unindo as equações, temos que para os coeficientes TE as equações A.9 e A.10.

$$
\begin{aligned}
& b_{n m} \xi_{n}(x)-\frac{1}{M} d_{n m} \psi_{n}(M x)=-G_{n m}^{T E} \psi_{n}(x) \\
& b_{n m} \xi^{\prime}{ }_{n}(x)-\frac{\mu_{1}}{\mu_{2}} d_{n m} \psi^{\prime}{ }_{n}(M x)=-G_{n m}^{T E} \psi_{n}^{\prime}(x)
\end{aligned}
$$


Igualmente, para os coeficientes TM, ficam:

$$
\begin{aligned}
& a_{n m} \xi_{n}(x)-\frac{\mu_{1}}{\mu_{2}} c_{n m} \psi_{n}(M x)=-G_{n m}^{T M} \psi_{n}(x) \\
& a_{n m} \xi_{n}^{\prime}(x)-\frac{1}{M} c_{n m} \psi_{n}^{\prime}(M x)=-G_{n m}^{T M} \psi_{n}^{\prime}(x)
\end{aligned}
$$

Para saber se as quantidades de observáveis variam com propriedades ópticas do meio, primeiro devemos obter as condições explícitas para a quantidade de espalhamento $a_{n m} b_{n m}$. As quatro equações lineares acima são resolvidas facilmente para coeficientes do campo dentro da partícula $\mu_{1} \approx \mu_{2}$, e assim, obtemos os coeficientes de espalhamento e interno da partícula.

$$
\begin{aligned}
& -a_{n}=\frac{a_{n m}}{G_{n m}^{T M}}=\frac{M \psi_{n}(M x) \psi_{n}{ }^{\top}(x)-\psi^{-}{ }_{n}(M x) \psi_{n}(x)}{\psi^{\prime}{ }_{n}(M x) \xi_{n}(x)-M \psi_{n}(M x) \xi^{\top}{ }_{n}(x)} \\
& -b_{n}=\frac{b_{n m}}{G_{n m}^{T E}}=\frac{M \psi^{{ }^{\prime}}{ }_{n}(M x) \psi_{n}(x)-\psi_{n}(M x) \psi^{{ }^{\prime}}{ }_{n}(x)}{\psi_{n}(M x) \xi^{\top}{ }_{n}(x)-M \psi^{{ }^{\prime}}{ }_{n}(M x) \xi_{n}(x)} \\
& -c_{n}=\frac{c_{n m}}{G_{n m}^{T E}}=\frac{\psi_{n}(x) \xi_{n}{ }_{n}(x)-\psi^{-}{ }_{n}(x) \xi_{n}(x)}{\psi^{{ }^{\prime}{ }_{n}(M x) \xi_{n}(x)-M \psi_{n}(M x) \xi^{{ }^{\prime}}{ }_{n}(x)}} \\
& -d_{n}=\frac{d_{n m}}{G_{n m}^{T E}}=\frac{\psi^{\top}{ }_{n}(x) \xi_{n}(x)-\psi_{n}(x) \xi^{\top}{ }_{n}(x)}{\psi_{n}(M x) \xi^{\prime}{ }_{n}(x)-M \psi^{-}{ }_{n}(M x) \xi_{n}(x)}
\end{aligned}
$$

Desta maneira, aplicado as funções de Bessel e Hankel, chegamos ao seu valor. 


\section{Apêndice B}

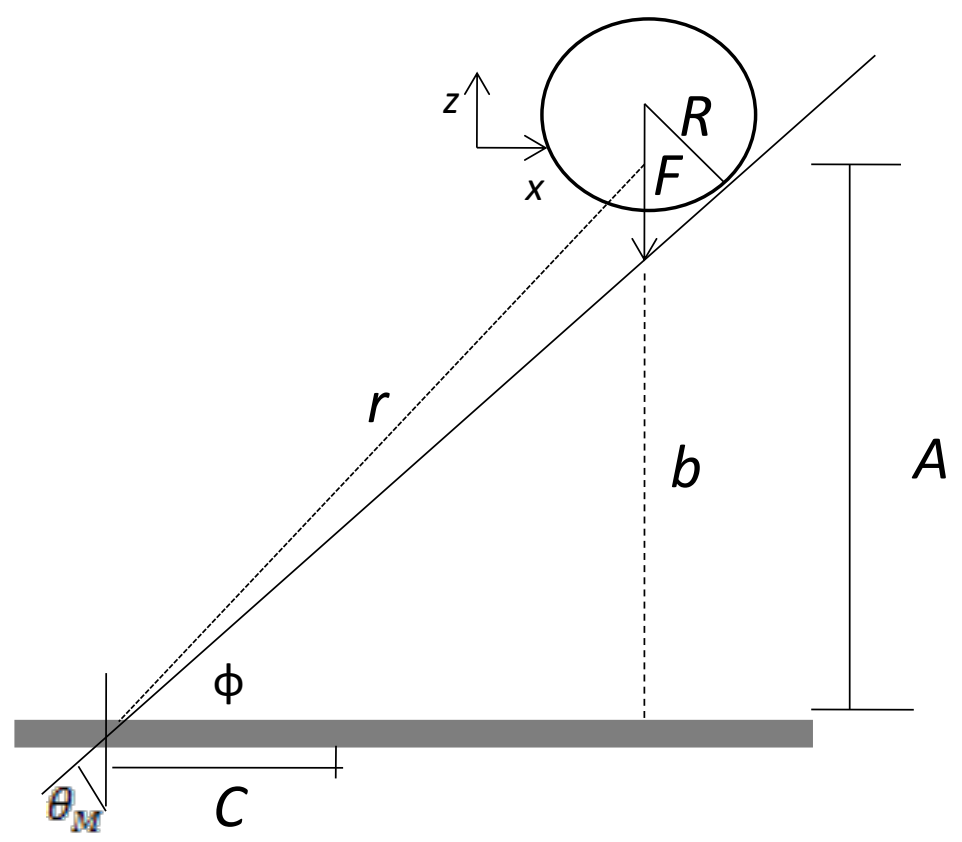

Figura B.1: Representação do raio e seu ângulo máximo $\theta_{M}$ usado no limite de integração da equação 2.58. Na figura é possível observar o raio tangente a esfera; $F$ representa à distância focal da objetiva e $\mathrm{C}$ a distância do ponto de incidência do raio até o centro da esfera; o retângulo escuro representa a lamínula; $\vec{r}$ o vetor que liga o foco da objetiva ao centro da esfera e $\vec{b}$ liga o raio refratado ao longo do eixo $\mathrm{z}^{\prime}$ ao raio da esfera; e, por fim, A representa a distância da lamínula ao centro da esfera.

O limite de raio atinge a esfera na Figura B.1, onde o ângulo $\theta_{M}$ representa o ângulo de incidência e $\phi$ o ângulo de refração, pois o raio passar pela lamínula. Nessa situação não temos outras forças atuando na esfera, apenas a força da pinça, logo, o centro está sendo deslocado em $z$. 
Para o ângulo máximo $\theta_{M}$, representado na Figura B.1, temos:

$$
\begin{aligned}
& \tan \phi=\frac{C}{A-r-b} \\
& \tan \theta_{M}=\frac{C}{A-r}
\end{aligned}
$$

Usando as leis de Snell-Decartes, podemos relacionar os ângulos.

$$
n_{v i d r o} \operatorname{sen} \theta_{M}=n_{2} \operatorname{sen} \phi
$$

Das equações B.1 e B.2 temos:

$$
\frac{\operatorname{sen} \phi \cos \theta_{M}}{\operatorname{sen} \theta_{M} \cos \phi}=\frac{A-r}{A-r-b}
$$

Logo, aplicando-a na equação B.3 chegamos a:

$$
\frac{n_{\text {vidro }}}{n_{2}} \frac{\cos \theta_{M}}{\cos \phi}=\frac{A-r}{A-r-b}
$$

Para eliminar a dependência de $\phi$, usamos, mais uma vez, a lei de Descartes.

$$
\operatorname{sen}^{2} \theta_{M}=\frac{\left(\frac{n_{2}}{n_{v i d r o}}\right)^{2}(A-r)^{2}-(A-r-b)^{2}}{(A-r)^{2}-(A-r-b)^{2}}
$$

Sendo que, de acordo com a Figura B.1, temos que b depende de $\phi$, assim:

$$
\operatorname{sen} \phi=\frac{d}{r+b}
$$

Então:

$$
b=\frac{n_{v i d r o}}{n_{2}} \frac{d}{\operatorname{sen} \theta_{M}}-r
$$

Desta forma, substituindo a equação B.8 na equação B.6, temos:

$$
\begin{gathered}
{\left[(A-r)^{2}-h^{2}\right] \operatorname{sen}^{4} \theta_{M}+2 d A\left(\frac{n_{2}}{n_{\text {vidro }}}\right) \operatorname{sen}^{3} \theta_{M}+\left[A^{2}-\left(\frac{n_{2}}{n_{\text {vidro }}}\right)^{2}\left(A^{2}-r^{2}\right)-\right.} \\
\left.\left(\frac{n_{2}}{n_{\text {vidro }}}\right)^{2} d^{2}\right] \operatorname{sen}^{2} \theta_{M}-2 A d\left(\frac{n_{2}}{n_{\text {vidro }}}\right) \operatorname{sen} \theta_{M}+\left(\frac{n_{2}}{n_{\text {vidro }}}\right)^{2} d^{2}=0
\end{gathered}
$$

E, desta forma, podemos resolver essa equação numericamente para valores fixos de $h, r, a$. 


\section{Apêndice C}

Nesse apêndice apresento o programa desenvolvido no software "Mathematica", versão 11.3, usado para realizar a simulação da força axial, com e sem deslocamento focal. Baseado no programa utilizado por NEVES (2006) ${ }^{1}$, sendo a primeira referência no capítulo 2 onde está o programa em que me baseei.

\section{Programa para o cálculo da força óptica axial}

Secção 1: Força axial sem deslocamento focal

(Inicialização do programa)

Clear["Global *"];

(definições das variáveis das equações; a distância focal, o comprimento de onda, o comprimento do feixe e o tamanho da bead estão em mícrons, o ângulo máximo está em radiano)

$\lambda=0.532$;

$\mathrm{NA}=1.30$

$\mathrm{f}=1700$;

$\omega b=1666$;

$\mathrm{d}=2.4 ; \mathrm{c}=3, \omega=1666$

$\mathrm{n} 2=1.3330$;

$\mathrm{n} 3=\operatorname{Sqrt}\left[1.03961212 * \lambda^{\wedge} 2 /\left(\lambda^{\wedge} 2-0.00600069867\right)+0.231792344 * \lambda^{\wedge} 2 /\left(\lambda^{\wedge} 2-\right.\right.$

$\left.0.0200179144)+1.01046945^{*} \lambda^{\wedge} 2 /\left(\lambda^{\wedge} 2-103.560653\right)+1\right]$;

$\mathrm{n} 1=\operatorname{Sqrt}\left[1+1.4435^{*} \lambda^{\wedge} 2 /\left(\lambda^{\wedge} 2-0.020216\right)\right]$;

$\mathrm{k}=(2 * \mathrm{Pi} * \mathrm{n} 2) / \lambda$;

$\mathrm{n} 0 \mathrm{n} 1=1 / \mathrm{Sqrt}[\mathrm{n} 3]$

$\mathrm{P}=2.100$

$\mathrm{eE} 2=(2 * \mathrm{P} *(0.7)) /(\mathrm{n} 2 * \mathrm{c} *$

$\left.\mathrm{Pi}^{*}\left(\omega^{2}\right)\right)^{*} 10^{\wedge}(-5)$,

$\alpha \max =\operatorname{ArcSin}[\mathrm{NA} / \mathrm{n} 2]$;

$\mathrm{kf}=\mathrm{k} * \mathrm{f}$;

$\mathrm{f} \omega=(\mathrm{f} / \omega \mathrm{b})^{*} 0.1$;

$f_{o}=1 /\left(f \omega^{*} \operatorname{Sin}[\alpha \max ]\right)$;

$\eta=\mathrm{n} 1 / \mathrm{n} 2$;

$\chi=\mathrm{Pi}^{*} \mathrm{~d} *(\mathrm{n} 2 / \lambda)$

\footnotetext{
${ }^{1}$ A. A. R. Neves. Força óptica em pinças ópticas: estudo teórico e experimental. 2006. 169 p. Tese (doutorado) -
} Universidade Estadual de Campinas, Instituto de Física GlebWataghin, Campinas, SP. 
$\mathrm{X}=1 ; \mathrm{Nmax}=\operatorname{IntegerPart}[\chi]+10$;

Nmax2=IntegerPart $[\chi]-40$;

Zini=-12;

Zinc $=0.3$;

Zfin $=15$;

$\operatorname{cosmax}=\operatorname{Cos}[\alpha \max ]$;

GridZ=Range[Zini,Zfin,Zinc];

GridN1=Range[1,Nmax];

GridN=Range[1,Nmax +1$]$;

GridZN=Outer[List,GridN,GridZ];

Zini=First[GridZ];

Zfin=Last[GridZ];

GridN12=Range[1,Nmax2];

GridN2=Range[1,Nmax2+1];

GridZN2=Outer[List,GridN2,GridZ];

Nessa parte o programa começa com uma inicialização que serve para limpar a memória das variáveis, se o desejado for reinicializar o programa novamente utilizam-se os comandos das linhas [1] a [3]. A linha 4 é utilizada para definir o diretório que o programa será executando, caso se queira importar algum arquivo para o programa e para importar ou exportar dados. As linhas de [5] a [36] definem as constantes do problema, as variáveis aqui utilizam os mesmos símbolos citados no capítulo 2 , como, por exemplo, $\lambda$ que refere-se ao comprimento de onda; com exceção de wb e $\alpha$ max, que no capítulo 2 são denotados por $w_{0}, \theta_{\text {max }}$, todos os valores estão em mícrons e radianos. Para a linha [20] definimos x como as variáveis usadas nas equações (A.23) e (A.24). Nas linhas [21] e [22] definimos os valores para $n$, onde, temos que a condição para $n$ é de $k r \gg n^{2}$,; então, consideramos as funções das equações (A.23) e (A.24), na forma não assinóptica, e, considerando a distância $r$ com comprimento de milímetros, que está comparado a mícrons, temos que, a condição se satisfaz; o número $n$ é escolhido a partir do valor menor $x$, pegando sua parte inteira, sendo que os números somados são colocados como melhor adição por tentativa e erro. Para as linhas [23] a [26] definimos o intervalo da força medida que vai de -12 a 15 mícrons.

A partir do ponto de equilíbrio, nas linhas [28] a [36] definimos as matrizes em forma de linha que serão utilizadas para o cálculo. Os valores são colocados em ordem, de acordo com o comando Range, sendo que, na linha [27] definimos o ângulo máximo $\theta_{\text {max }}$ utilizado para o cálculo da integral apresentada nas equações 3.3, 3.8, 3.18, 3.19, 3.24, e 3.25.

$\mathrm{Na}$ linha [14] temos a constante de normalização que depende da potência do laser. 
(Cálculo das integrais do coeficiente de incidência do feixe)

$\mathrm{I} 0[\mathrm{n}, \mathrm{xo}]:=\mathrm{Block}\left[\{\right.$ func $\}$, func $\left[\left(\mathrm{x} \_\right)\right.$NumericQ $]:=\left(\operatorname{Sqrt}\left[\mathrm{x} /\left(1-\mathrm{x}^{\wedge} 2\right)\right]^{*}\left(\operatorname{LegendreP}[\mathrm{n}, 1, \mathrm{x}]^{*}(1-\mathrm{x})-\right.\right.$ $\mathrm{n}^{*}(\mathrm{n}+1)^{*} \operatorname{Sqrt}\left[1-\mathrm{x}^{\wedge} 2\right]^{*}$ LegendreP $\left.\left.[\mathrm{n}, \mathrm{x}]\right)\right) /\left(\mathrm{E}^{\wedge}((1-\right.$

$\left.\left.\left.\mathrm{x}^{\wedge} 2\right)^{*} \mathrm{f} \omega^{\wedge} 2\right)^{*} \mathrm{E}^{\wedge}\left(\mathrm{I}^{*} \mathrm{k}^{*} \mathrm{xo} \mathrm{*}^{*} \mathrm{x}\right)\right)$; NIntegrate[func $[\mathrm{x}],\{\mathrm{x}, \cos m a x, 1\}$, AccuracyGoal-> 7, Compiled-

$>$ False,MaxRecursion- $>100]]$;

$\mathrm{MI} 0=A p p l y[\mathrm{I} 0[\# 1, \# 2] \&, \operatorname{GridZN},\{2\}]$;

Na linha [37] integramos nos limites que vão de cosmax a 1, na linha [38] aplicam-se os valores listados no início do programa de $n$ e $m$.

\section{(Cálculo dos expoentes de Mie ou de Espalhamento)}

$\psi\left[\mathrm{n}_{-}, \mathrm{y}\right]=\mathrm{y} *$ SphericalBesselJ $[\mathrm{n}, \mathrm{y}]$;

$\zeta\left[\mathrm{n}_{-}, \mathrm{y}_{-}\right]=\mathrm{y} *$ SphericalHankelH1[n,y];

$\mathrm{D} \psi\left[\mathrm{n}_{-}, \mathrm{y} \_\right]=\mathrm{D}[\psi[\mathrm{n}, \mathrm{y}], \mathrm{y} ;$

$\left.\mathrm{D} \zeta\left[\mathrm{n}_{-}, \mathrm{y}\right]\right]=\mathrm{D}[\zeta[\mathrm{n}, \mathrm{y}], \mathrm{y}]$;

$\mathrm{AB}\left[\mathrm{n} \_, \mathrm{xx}, \mathrm{mm}\right]=$ Module$[\{\mathrm{vPsi}, \mathrm{vPsiM}, \mathrm{vZeta}, \mathrm{vDPsi}, \mathrm{vDPsiM}, \mathrm{vDZeta}\}, \mathrm{vPsi}=\psi[\mathrm{n}, \mathrm{xx}] ; \mathrm{vPsiM}=$ $\psi\left[\mathrm{n}, \mathrm{mm} \mathrm{m}^{*} \mathrm{xx}\right]$;

$\mathrm{vZeta}=\zeta[\mathrm{n}, \mathrm{xx}]$;

$\mathrm{vDPsi}=\mathrm{D} \psi[\mathrm{n}, \mathrm{xx}] ; \mathrm{vDPsiM}=\mathrm{D} \psi[\mathrm{n}, \mathrm{mm} * \mathrm{xx}] ; \mathrm{vDZeta}=\mathrm{D} \zeta[\mathrm{n}, \mathrm{xx}]$

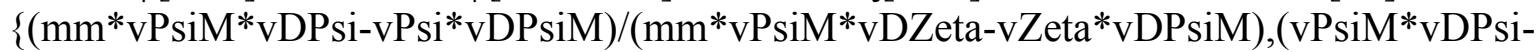
$\left.\mathrm{mm}^{*}{ }^{\mathrm{v} P s i}{ }^{*} \mathrm{vDPsiM}\right) /\left(\mathrm{vPsiM}{ }^{*} \mathrm{vDZ}\right.$ eta-mm*vZeta*vDPsiM $\left.\left.)\right\}\right]$; $\{\mathrm{At}, \mathrm{Bt}\}=\mathrm{AB}[\mathrm{GridN}, \chi, \eta]$

Nas linhas de [39] a [46] escrevemos as funções A.23 e A.24, que são os coeficientes de Mie no software, e, na linha [46] aplicamos os valores definidos no início, onde usamos a equação para função direta esférica de Henkel e Bessel. No programa utilizado como referência ${ }^{1}$, essa equação não estava presente, pois ela utilizava a versão 5.2 do software que não possuía essas funções, sendo usadas identidades matemática para simulá-las. Com a versão atual, elas não foram necessárias.

\section{(Cálculo da secção de choque da força de pressão de radiação)}

$\mathrm{CpZ}\left[\mathrm{n} \_\right.$,xo $]:=\left(1 /(\mathrm{n}+1)^{\wedge} 3\right) * \operatorname{Re}[(\operatorname{At}[[\mathrm{n}]]+$ Conjugate$[\operatorname{At}[[\mathrm{n}+1]]]-$

$2 * \operatorname{At}[[\mathrm{n}]] *$ Conjugate[At $[[\mathrm{n}+1]]]+\mathrm{Bt}[[\mathrm{n}]]+$ Conjugate$[\mathrm{Bt}[[\mathrm{n}+1]]]-$

$2 * \operatorname{Bt}[[\mathrm{n}]] *$ Conjugate$[\mathrm{Bt}[[\mathrm{n}+1]]]) * \operatorname{MI} 0[[\mathrm{n}, \mathrm{xo}]] *$ Conjugate[MI0[[n+1,xo]]]];

SetAttributes[CpZ,Listable];

Na linha [48], aplicamos a integral e os coeficientes de Mie ao coeficiente de impacto que é a equação 3.9, e na linha [49] deixamos o coeficiente de impacto numa forma de lista. 


\section{(Cálculo da força axial)}

ForcaZ[xo ]:=(1/2)*Pi*(f/10^6)^2*n0n1*Tr[CpZ[Range[Nmax],xo]];

ForcaAxial=(ForcaZ[\#1]\&)/@Range[Length[GridZ]];

fForcaAxial=ListInterpolation[Re[ForcaAxial], $\{\{$ Zini,Zfin $\}\}]$;

data $=$ Transpose $[\{$ GridZ,Re[ForcaAxial $]\}]$;

ListPlot[data,Joined->False,PlotRange- $>$ All]

E, por fim, o cálculo da força na linha [50] é somado em relação a $n$ na linha [51] e, aplicando o comprimento de $15 \mu \mathrm{m}$ a $z_{0}$ na linha [52], construímos uma matriz ou tabela com esses elementos na linha [53] que é usada para criar o gráfico da posição pela força radial na linha [54] através do comando ListPlot.

Secção 2: Força axial com deslocamento focal

$1=10$

$\mathrm{k} 2=\mathrm{k} *(\mathrm{n} 2 / \mathrm{n} 3)$

$\mathrm{z}[\mathrm{x}] \mathrm{]}=\operatorname{Sqrt}\left[1-\left(\left((\mathrm{n} 3 / \mathrm{n} 2)^{\wedge} 2\right)^{*}\left(\left(1-\mathrm{x}^{\wedge} 2\right)^{\wedge} 2\right)\right)\right]$;

$\operatorname{tp}[\mathrm{x}]:=(2 * \mathrm{x}) /((\mathrm{n} 2 / \mathrm{n} 3) * \mathrm{x}+\mathrm{z}[\mathrm{x}])$

ts $[\mathrm{x}]:=(2 * \mathrm{x}) /(\mathrm{x}+\mathrm{z}[\mathrm{x}] *(\mathrm{n} 2 / \mathrm{n} 3))$

(Cálculo da nova integral com as novas funções tp, ts, e uma nova variável $\mathrm{z}=\cos (\alpha 2)$, que constituem a equação com deslocamento focal; equações 2.110 a 2.112)

$\mathrm{I} 02[\mathrm{n}, \mathrm{xo}]:=$

Block $[\{$ func $\}$,

func $[(\mathrm{x})$ ? Numeric $\mathrm{Q}]:=$

$\operatorname{Sqrt}[\mathrm{x}]^{*}\left(\operatorname{LegendreP}[\mathrm{n}, 1, \mathrm{z}[\mathrm{x}]]^{*}\left(\operatorname{ts}[\mathrm{x}]-\operatorname{tp}[\mathrm{x}]^{*} \mathrm{z}[\mathrm{x}]\right)-\right.$

$\mathrm{n}^{*}(\mathrm{n}+1) * \operatorname{Sqrt}\left[\left(1-\mathrm{x}^{\wedge} 2\right)\right]^{*} \operatorname{tp}[\mathrm{x}]^{*}$

LegendreP[n, $\mathrm{z}[\mathrm{x}]]) /\left(\mathrm{E}^{\wedge}\left(\left(1-\mathrm{x}^{\wedge} 2\right) * \mathrm{f}\left[[\mathrm{Omega}]^{\wedge} 2\right)^{*}\right.\right.$

$\left.\mathrm{E}^{\wedge}\left(\mathrm{I}^{*}\left(\left(\mathrm{k}^{*} \mathrm{x} * \mathrm{l}\right)+(\mathrm{k} 2 *(\mathrm{xo}-1) * \mathrm{z}[\mathrm{x}])\right)\right)\right)$

NIntegrate $[((n-1) ! /(n+1) !) *$ func $[x],\{x, \operatorname{cosmax}, 1\}$,

AccuracyGoal $->1$, Compiled $->$ False, WorkingPrecision $->30$,

MaxRecursion $->$ 30,

Method -> \{"OscillatorySelection", "SymbolicProcessing" -> 0\}]];

MI20=Apply[I02[\#1,\#2]\&,GridZN2,\{2\}];

(Cálculo da seção de pressão de radiação com fenômeno de deslocamento focal)

$\operatorname{CpZ3}\left[\mathrm{n}_{-}, \mathrm{xo}\right]:=\left(\left(1 /(\mathrm{n}+1)^{\wedge} 2\right)^{*} \operatorname{Sqrt}[\mathrm{n} /(\mathrm{n}+2)]\right) * \operatorname{Re}[(\operatorname{At}[[\mathrm{n}]]+\operatorname{Conjugate}[\operatorname{At}[[\mathrm{n}+1]]]-$ $2 * \operatorname{At}[[\mathrm{n}]] *$ Conjugate[At $[[\mathrm{n}+1]]]+\mathrm{Bt}[[\mathrm{n}]]+\operatorname{Conjugate}[\mathrm{Bt}[[\mathrm{n}+1]]]-$ $2 * \mathrm{Bt}[[\mathrm{n}]] *$ Conjugate $[\mathrm{Bt}[[\mathrm{n}+1]]]) * \operatorname{MI} 20[[\mathrm{n}, \mathrm{xo}]] *$ Conjugate[MI20[[n+1,xo] $]]]$; SetAttributes[CpZ2,Listable]; 
(Cálculo da força axial de radiação com fenômeno de deslocamento focal)

ForcaZ2[xo]: $=(1 / 2) * \mathrm{Pi}^{*}\left(\mathrm{f} / 10^{\wedge} 6\right)^{\wedge} 2 *(\mathrm{n} 2 / \mathrm{n} 3)^{*} \operatorname{Tr}[\mathrm{CpZ} 2[\mathrm{Range}[\mathrm{Nmax} 2], \mathrm{xo}]]$;

ForcaAxial2=(ForcaZ2[\#1]\&)/@Range[Length[GridZ]];

fForcaAxial2=ListInterpolation[Re[ForcaAxial2], $\{\{$ Zini,Zfin $\}\}]$;

ListPlot[data2,Joined-> True,PlotRange->All]

Das linha [55] a [68] temos o cálculo da equaçao da força axial levando em conta o delocamento focal, utilizando a sequações 3.8 e 3.12, onde temos o mesmo processo para o caso não focal, apenas adicionamos as novas equações que são as correções que corresponde a linha [55], o deslocamento do foco em mícrons; na linha [58] e [59] as funções tp e ts; e na linha [57] a nova variável integrável z[x]. Na linha [56] temos um novo $k_{2}$, que depende de $k$ , desta forma, essa variáveis são escritas como $d, t^{p}, t^{s}, x_{2}=\cos \left[\alpha_{2}\right]$, e $k_{2}$, apresentados nas equações do capítulo 3 . Onde a equação 3.8 corresponde à linha [60], a equação 3.9 à linha [62], e a equação 3.12 à linha [64]. Foi necessário mudar o arredondamento para 2 e o intervalo x0 calculado que é $[-1,1]$ e não mais $[-12,15]$, como no anterior, pois a função possui uma tremenda não linearidade que toma muito esforço computacional para calcular, sendo que esse tópico já discutido no Capítulo 5.

Secção 3: Derivação da força perto da origem para obtenção teórica kz da armadilha óptica (Cálculo de k sem deslocamento focal)

$\operatorname{IOd}\left[\mathrm{n}_{-}\right]:=\mathrm{Block}\left[\{\right.$ func $\}$,func $\left[\left(\mathrm{x}_{-}\right)\right.$? NumericQ] $:=-\mathrm{I} \mathrm{E}^{\wedge}\left(-\mathrm{f}\left[[\text { Omega }]^{\wedge} 2\left(1-\mathrm{x}^{\wedge} 2\right)\right) \mathrm{k} \mathrm{x} \mathrm{Sqrt}[\mathrm{x} /(1-\right.$ $\left.\left.x^{\wedge} 2\right)\right]\left(-n(1+n) \operatorname{Sqrt}\left[1-x^{\wedge} 2\right]\right.$ LegendreP[n,x]+(1-x)

LegendreP[n,1,x]);NIntegrate[func $[\mathrm{x}],\{\mathrm{x}, \operatorname{cosmax}, 1\}$,AccuracyGoal- $>$ 7,Compiled-

$>$ False,MaxRecursion->100]];

I0d'=Table[I0d[n], \{n,1,Nmax+1\}];

$\mathrm{I} 03\left[\mathrm{n} \_\right.$]:=Block$\left[\{\right.$func $\}$,func $\left[\left(\mathrm{x} \_\right)\right.$?NumericQ $]:=\left(\operatorname{Sqrt}[\mathrm{x}]^{*}\left(\operatorname{LegendreP}[\mathrm{n}, 1, \mathrm{x}]^{*}(1-\mathrm{x})-\right.\right.$ $\mathrm{n}^{*}(\mathrm{n}+1) * \operatorname{Sqrt}\left[1-\mathrm{x}^{\wedge} 2\right]^{*}$ LegendreP $\left.\left.[\mathrm{n}, \mathrm{x}]\right)\right) /\left(\mathrm{E}^{\wedge}((1-\right.$ $\left.\left.\mathrm{x}^{\wedge} 2\right)^{*} \mathrm{f}\left(\omega^{\wedge} 2\right)\right) ;$ NIntegrate[func $[\mathrm{x}],\{\mathrm{x}, \cos m a x, 1\}$, AccuracyGoal->7,Compiled$>$ False,MaxRecursion- $>100]]$;

$\mathrm{I} 03^{\prime}=$ Table $[\mathrm{I} 03[\mathrm{n}],\{\mathrm{n}, 1, \mathrm{Nmax}+1\}]$

$\mathrm{CpZd}[\mathrm{n}]:=\left(1 /(\mathrm{n}+1)^{\wedge} 3\right) * \operatorname{Re}[(\operatorname{At}[[\mathrm{n}]]+$ Conjugate$[\operatorname{At}[[\mathrm{n}+1]]]-$

$2 * \operatorname{At}[[n]] *$ Conjugate[At $[[n+1]]]+\operatorname{Bt}[[\mathrm{n}]]+$ Conjugate[Bt[[n+1]]]-

$2 * \mathrm{Bt}[[\mathrm{n}]] *$ Conjugate $[\mathrm{Bt}[[\mathrm{n}+1]]]) *\left(\operatorname{IOd} \mathrm{d}^{\prime}[[\mathrm{n}]] *\right.$ Conjugate $\left[\mathrm{I03}{ }^{\prime}[[\mathrm{n}+1]]\right]+\mathrm{I03}{ }^{\prime}[[\mathrm{n}]]^{*}$ Conjugate $[\mathrm{IOd}$ $'[[n+1]]])]$; 
SetAttributes[CpZd,Listable];

$\mathrm{Kz}=\mathrm{eE} 2 *(1 / 2) * \mathrm{Pi}^{*}\left(\mathrm{f} / 10^{\wedge} 6\right)^{\wedge} 2 *(\mathrm{n} 0 \mathrm{n} 1)^{\wedge} 2 * \mathrm{Sum}[\mathrm{CpZd}[\mathrm{n}],\{\mathrm{n}, 1, \mathrm{Nmax}\}]^{*} 10^{\wedge} 6$

Nessa parte, simulamos os valores de $k$, primeiro sem o deslocamento focal, onde calculamos a derivada de I0 para xô $=0$, e calculamos sua integral nas linhas [69] e [70]. E, depois, calculamos Io para o ponto $x \hat{0}=0$ nas linhas [71] e [72]. Usamos as integrais no cálculo da somatória da derivada na linha [73], tornando esses valores em uma lista na linha [74], e, na linha [75] fazemos suas somatórias vezes as constantes, onde $10^{6}$ se dá devido ao falto de $k$ cair do denominador, sendo esse o resultado $\mathrm{k}$ na direção axial sem deslocamento focal.

\section{(Cálculo de k com deslocamento focal)}

ç[xo]: $=\mathrm{IE}^{\wedge}\left(-\mathrm{f} \backslash[\mathrm{Omega}]^{\wedge} 2\left(1-\mathrm{x}^{\wedge} 2\right)-\mathrm{I}(\mathrm{k} 1 \mathrm{x}-\mathrm{k} 21 \mathrm{z}[\mathrm{x}])\right) \mathrm{k} 2 \operatorname{Sqrt}[\mathrm{x}] \mathrm{z}[\mathrm{x}]\left(-\mathrm{n}(1+\mathrm{n}) \operatorname{Sqrt}\left[1-\mathrm{x}^{\wedge} 2\right]\right.$ LegendreP $[\mathrm{n}, \mathrm{z}[\mathrm{x}]] \operatorname{tp}[\mathrm{x}]+$ LegendreP[n,1,z[x]] (ts[x]-tp[x] z[x]));

$c^{\prime}[0]$

$-\mathrm{E}^{\wedge}\left(-\mathrm{f} \backslash[\mathrm{Omega}]^{\wedge} 2\left(1-\mathrm{x}^{\wedge} 2\right)\right) \mathrm{k} \mathrm{x} \operatorname{Sqrt}\left[\mathrm{x} /\left(1-\mathrm{x}^{\wedge} 2\right)\right]\left(-\mathrm{n}(1+\mathrm{n}) \operatorname{Sqrt}\left[1-\mathrm{x}^{\wedge} 2\right]\right.$ LegendreP[n,x]+(1-x) LegendreP $[\mathrm{n}, 1, \mathrm{x}])$

$\operatorname{Ik} 02\left[\mathrm{n}_{-}\right]:=\mathrm{B} \operatorname{lock}\left[\{\right.$ func $\}$, func $\left[\left(\mathrm{x} \_\right)\right.$NumericQ $]:=\mathrm{I} \mathrm{E} \mathrm{E}^{\wedge}\left(-\mathrm{f} \backslash[\text { Omega }]^{\wedge} 2\left(1-\mathrm{x}^{\wedge} 2\right)-\mathrm{I}(\mathrm{k} 1 \mathrm{x}-\mathrm{k} 21 \mathrm{z}[\mathrm{x}])\right)$ k2 Sqrt $[\mathrm{x}] \mathrm{z}[\mathrm{x}]\left(-\mathrm{n}(1+\mathrm{n}) \operatorname{Sqrt}\left[1-\mathrm{x}^{\wedge} 2\right]\right.$ LegendreP[n,z[x]] tp[x]+LegendreP[n,1,z[x]] (ts[x]$\operatorname{tp}[\mathrm{x}] \mathrm{z}[\mathrm{x}]))$; NIntegrate $[((\mathrm{n}-1) ! /(\mathrm{n}+1) !) *$ func $[\mathrm{x}],\{\mathrm{x}, \operatorname{cosmax}, 1\}$,AccuracyGoal- $>1$, Compiled$>$ False, WorkingPrecision- $>30$, MaxRecursion- $>30$, Method-

$>\{$ "OscillatorySelection","SymbolicProcessing"->0\}]];

$\operatorname{I0d} 2^{\prime}=$ Table[Ik02[n], \{n,1,Nmax+1\}];

I02focal[n_]:=Block $\left[\{\right.$ func $\}$, func $\left[\left(\mathrm{x} \_\right)\right.$? NumericQ $]:=\operatorname{Sqrt}[\mathrm{x}] *\left(\operatorname{LegendreP}[\mathrm{n}, 1, \mathrm{z}[\mathrm{x}]]^{*}(\operatorname{ts}[\mathrm{x}]-\right.$ $\left.\operatorname{tp}[\mathrm{x}]^{*} \mathrm{z}[\mathrm{x}]\right)-\mathrm{n}^{*}(\mathrm{n}+1)^{*} \operatorname{Sqrt}\left[\left(1-\mathrm{x}^{\wedge} 2\right)\right]^{*} \operatorname{tp}[\mathrm{x}]^{*}$ LegendreP $\left.[\mathrm{n}, \mathrm{z}[\mathrm{x}]]\right) /\left(\mathrm{E}^{\wedge}((1-\right.$ $\left.\left.\mathrm{x}^{\wedge} 2\right) * \mathrm{f}\left[[\mathrm{Omega}]^{\wedge} 2\right)^{*} \mathrm{E}^{\wedge}\left(\mathrm{I}^{*}\left(\left(\mathrm{k}^{*} \mathrm{x} * 1\right)+(\mathrm{k} 2 *(-1) * \mathrm{z}[\mathrm{x}])\right)\right)\right) ;$ NIntegrate $[((\mathrm{n}-$ $1) ! /(\mathrm{n}+1) !) *$ func $[\mathrm{x}],\{\mathrm{x}$, cosmax, 1$\}$,AccuracyGoal- $>1$, Compiled- $>$ False, WorkingPrecision$>30$, MaxRecursion->30,Method->\{"OscillatorySelection","SymbolicProcessing"->0 $\}]$;;

$\mathrm{I} 02^{\prime}=$ Table $[\mathrm{Ik} 02[\mathrm{n}],\{\mathrm{n}, 1, \mathrm{Nmax}+1\}]$

$\operatorname{CpZd} 2\left[\mathrm{n} \_\right]:=\left(\left(1 /(\mathrm{n}+1)^{\wedge} 3\right)^{*}\left(1 /(\mathrm{n}+1)^{\wedge} 2\right)^{*} \operatorname{Sqrt}[\mathrm{n} /(\mathrm{n}+1)]\right) * \operatorname{Re}[(\operatorname{At}[[\mathrm{n}]]+\operatorname{Conjugate}[\operatorname{At}[[\mathrm{n}+1]]]-$ $2 * \operatorname{At}[[\mathrm{n}]] *$ Conjugate[$[\mathrm{At}[[\mathrm{n}+1]]]+\mathrm{Bt}[[\mathrm{n}]]+$ Conjugate$[\mathrm{Bt}[[\mathrm{n}+1]]]-$ $2 * \operatorname{Bt}[[\mathrm{n}]] *$ Conjugate $[\mathrm{Bt}[[\mathrm{n}+1]]]) *(((\mathrm{n}+1) ! /(\mathrm{n}-1) !) \wedge 2) *(((\mathrm{n}+1) ! /(\mathrm{n}-$ $\left.1) !)^{\wedge} 2\right) \operatorname{IOd} 2 \backslash . \mathrm{b} 4[[\mathrm{n}]]^{*}$ Conjugate$[\mathrm{I} 02 \backslash . \mathrm{b} 4[[\mathrm{n}+1]]]+\mathrm{I} 02 \backslash . \mathrm{b} 4[[\mathrm{n}]]^{*}$ Conjugate$\left.\left.[\mathrm{IOd} 2 \backslash . \mathrm{b} 4[[\mathrm{n}+1]]]\right)\right]$;

SetAttributes[CpZd2,Listable]; 
Nas linhas [76] e [77] definimos a equação de integração com deslocamento focal I02 da linha [60] para calcular sua derivada em relação xô $=0$, depois integramos a linha [78] no mesmo processo descrito no cálculo de $k$ sem deslocamento focal. Isso nos fornece $\mathrm{kz}$ simulado levando em conta o deslocamento focal na linha [85]. 


\section{Apêndice D}

Neste apêndice apresento o programa desenvolvido no software "Mathematica", versão 11.3, usado para realizar a simulação da força radial, com e sem deslocamento focal. Baseado no programa utilizado por NEVES (2006) ${ }^{2}$.

\section{Programa para o cálculo da força óptica radial.}

(Inicialização do programa)

Clear["Global *"];

Off[General::spell];

Off[General::spell1];

(definições das variáveis das equações; a distância focal, o comprimento de onda, o comprimento do feixe e o tamanho da bead estão em mícrons, o ângulo máximo está em radiano)

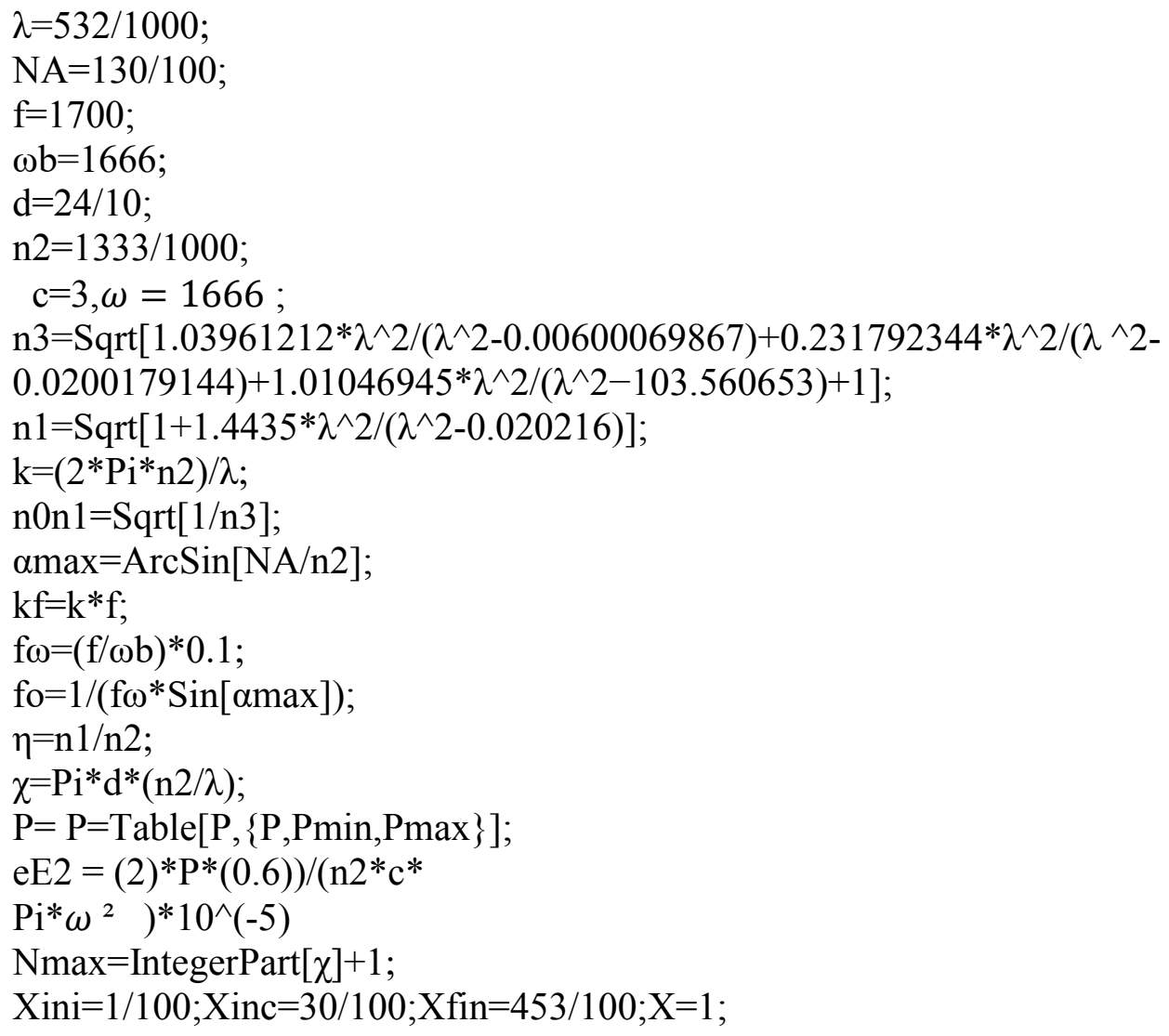

Nmax $=$ IntegerPart $[\chi]+1$;

$\mathrm{Xini}=1 / 100 ; \mathrm{Xinc}=30 / 100 ; \mathrm{Xfin}=453 / 100 ; \mathrm{X}=1$;

${ }^{2}$ A. A. R. Neves. Força óptica em pinças ópticas: estudo teórico e experimental. 2006.169 p. Tese (doutorado) - Universidade Estadual de Campinas, Instituto de Física GlebWataghin, Campinas, SP. 
$\operatorname{cosmax}=\operatorname{Cos}[\alpha \max ] ; \operatorname{GridX}=$ Range$[\mathrm{Xini}, \mathrm{Xfin}, \mathrm{Xinc}]$;

GridN=Range[Nmax];

GridN1=Range $[\mathrm{Nmax}+1]$;

GridXN=Table $[\{\mathrm{n}, \mathrm{m}, \mathrm{xx}\},\{\mathrm{n}, 1, \mathrm{Nmax}+1\},\{\mathrm{m}, 0, \mathrm{n}\},\{\mathrm{xx}, \mathrm{Xini}, \mathrm{Xfin}, \mathrm{Xinc}\}] ;$

Xini=First $[$ GridX];

Xfin=Last[GridX];

O programa é quase idêntico, em seu começo, ao caso axial explicado no Apêndice C. No entanto, o programa muda anotação para a distância radial percorrida, na linha [24], e o número de variação n que é menor, pois as equações são maiores e o tempo de computacional também, então, escolhemos um n max menor, onde os valores são colocados na forma de listra a matriz, igual ao da secção 3.2.

\section{(Cálculo dos coeficientes de espalhamento ou coeficientes de Mie)}

$\psi\left[\mathrm{n} \_\mathrm{y}\right]=\mathrm{y} *$ SphericalBesselJ[n,y];

$\zeta\left[\mathrm{n}_{-}, \mathrm{y} \_\right]=\mathrm{y}^{*}$ SphericalHankelH1[n,y];

$\mathrm{D} \psi\left[\mathrm{n}_{-}, \mathrm{y}\right]=\mathrm{D}[\psi[\mathrm{n}, \mathrm{y}], \mathrm{y}] ;$

$\mathrm{D} \zeta\left[\mathrm{n}_{-}, \mathrm{y}_{-}\right]=\mathrm{D}[\zeta[\mathrm{n}, \mathrm{y}], \mathrm{y}]$;

$\mathrm{AB}\left[\mathrm{n} \_\mathrm{xx} \_, \mathrm{mm}\right]=$ Module[ $\{\mathrm{vPsi}, \mathrm{vPsiM}, \mathrm{vZeta}, \mathrm{vDPsi}, \mathrm{vDPsiM}, \mathrm{vDZeta}\}, \mathrm{vPsi}=\psi[\mathrm{n}, \mathrm{xx}] ; \mathrm{v}$

PsiM $=\psi\left[n, m m^{*} x x\right]$;

$\mathrm{vZeta}=\zeta[\mathrm{n}, \mathrm{xx}]$;

vDPsi $=\mathrm{D} \psi[\mathrm{n}, \mathrm{xx}] ; \mathrm{vDPsiM}=\mathrm{D} \psi[\mathrm{n}, \mathrm{mm} * \mathrm{xx}] ; \mathrm{vDZeta}=\mathrm{D} \zeta[\mathrm{n}, \mathrm{xx}]$;

$\left\{\left(\mathrm{mm} * \mathrm{vPsiM} * \mathrm{vDPsi}-\mathrm{vPsi}{ }^{*} \mathrm{vDPsiM}\right) /(\mathrm{mm} * \mathrm{vPsiM} * \mathrm{vDZ}\right.$ eta-

vZeta*vDPsiM),(vPsiM*vDPsi-mm*vPsi*vDPsiM)/(vPsiM*vDZetamm*VZeta*vDPsiM) $\}$;

$\{\mathrm{At}, \mathrm{Bt}\}=\mathrm{AB}[\mathrm{GridN}, \chi, \eta]$

0 cálculo dos coeficientes de Mie é idêntico ao cálculo do caso axial.

\section{(Cálculo das Integrais dos coeficientes de incidência do feixe)}

$\mathrm{I} 1\left[\mathrm{n} \_\mathrm{m} \_, \mathrm{xo}\right]$ ]:=Block$\left[\{\right.$ func $\}$, func $\left[\left(\mathrm{x} \_\right)\right.$NumericQ $]:=\operatorname{Sqrt}[\mathrm{x}]^{*}\left(\mathrm{~m} *\right.$ LegendreP $[\mathrm{n}, \mathrm{m}, \mathrm{x}]^{*}$ $\left(\left(\mathrm{x}^{*} \operatorname{BesselJ}\left[\mathrm{m}+1, \mathrm{k}^{*} \mathrm{xo} * \operatorname{Sqrt}\left[1-\mathrm{x}^{\wedge} 2\right]\right]\right) / \operatorname{Sqrt}\left[1-\mathrm{x}^{\wedge} 2\right]\left(\mathrm{m}^{*} \operatorname{Besse} 1 \mathrm{~J}\left[\mathrm{~m}, \mathrm{k}^{*} \mathrm{xo} \mathrm{o}^{*} \operatorname{Sqrt}[1-\right.\right.\right.$ $\left.\left.\left.\left.\mathrm{x}^{\wedge} 2\right]\right]\right) /\left(\mathrm{k}^{*} \mathrm{xo}^{*}(\mathrm{x}+1)\right)\right)-(\mathrm{m}+\mathrm{n}) *(-\mathrm{m}+\mathrm{n}+1) *$ LegendreP[n,m$1, \mathrm{x}]^{*}\left(\left(\mathrm{~m} * \operatorname{BesselJ}\left[\mathrm{m}, \mathrm{k}^{*} \mathrm{xo}^{*} \operatorname{Sqrt}\left[1-\mathrm{x}^{\wedge} 2\right]\right]\right) /\left(\mathrm{k}^{*} \mathrm{xo}^{*} \operatorname{Sqrt}\left[1-\mathrm{x}^{\wedge} 2\right]\right) \operatorname{BesselJ}\left[\mathrm{m}+1, \mathrm{k}^{*} \mathrm{xo}^{*} \operatorname{Sqrt}[1-\right.\right.$ $\left.\left.\left.\left.\mathrm{x}^{\wedge} 2\right]\right]\right)\right) / \mathrm{E}^{\wedge}\left(\mathrm{f} \omega{ }^{\wedge} 2^{*}\left(1-\mathrm{x}^{\wedge} 2\right)\right)$;

NIntegrate $[((n-m) ! ! *$ func $[x]) /(m+n) ! !,\{x, \operatorname{cosmax}, 1\}$,AccuracyGoal- $>4$, Compiled$>$ False,MaxRecursion- $>100$

, Method->\{"OscillatorySelection","SymbolicProcessing"->0\}]];

MI1=Apply[I1[\#1,\#2,\#3]\&,GridXN, $\{3\}]$; 
$\mathrm{I} 2[\mathrm{n}, \mathrm{m}, \mathrm{xo}]:=$

Block $[\{$ func $\}$,

func $\left[\left(\mathrm{x}_{-}\right)\right.$? NumericQ] :=

$\operatorname{Sqrt}[\mathrm{x}]^{*}($ LegendreP $[\mathrm{n}, \mathrm{m}$,

$\mathrm{x}] *\left(\left(\mathrm{~m} * \operatorname{BesselJ}\left[\mathrm{m}, \mathrm{k}^{*} \mathrm{xo}^{*} \operatorname{Sqrt}\left[1-\mathrm{x}^{\wedge} 2\right]\right]\right) /\left(\mathrm{k}^{*} \mathrm{xo}^{*}(\mathrm{x}+1)\right)-\right.$

BesselJ $\left[\mathrm{m}+1, \mathrm{k}^{*} \mathrm{xo}{ }^{*} \operatorname{Sqrt}\left[1-\mathrm{x}^{\wedge} 2\right]\right] /$

$\left.\operatorname{Sqrt}\left[1-x^{\wedge} 2\right]\right)-\left((m+n) *(-m+n+1)^{*}\right.$

LegendreP[n, m - 1, x] $]^{*}$ BesselJ $\left.\left[\mathrm{m}, \mathrm{k}^{*} \mathrm{xo}^{*} \operatorname{Sqrt}\left[1-\mathrm{x}^{\wedge} 2\right]\right]\right) /\left(\mathrm{k}^{*}\right.$

xo*Sqrt $\left.\left.\left[1-\mathrm{x}^{\wedge} 2\right]\right)\right) / \mathrm{E}^{\wedge}\left(\mathrm{f}[\mathrm{O} \text { Omega }]^{\wedge} 2^{*}\left(1-\mathrm{x}^{\wedge} 2\right)\right)$;

NIntegrate $\left[\left((\mathrm{n}-\mathrm{m}) ! !^{*}\right.\right.$ func $\left.[\mathrm{x}]\right) /(\mathrm{m}+\mathrm{n}) !$ !, $\{\mathrm{x}, \operatorname{cosmax}, 1\}$,

AccuracyGoal -> 4, Compiled -> False, MaxRecursion -> 100,

Method -> \{"OscillatorySelection", "SymbolicProcessing" -> 0 \}]];

MI2 = Apply[I2[\#1,\#2,\#3]\&, GridXN, $\{3\}]$;

Nas linhas [40] e [43] escrevemos a integral, equaçao 3.18,319, nas linhas [41] e [44] no utilizamos o cômodo para o cálculo da integral numérica, temos os comandos para recursão, precisão, e procissão de ponto flutuante, e métodos que não tratam ela como processo simbólico e uma seleção das oscilações, se tiver altas oscilações. Já nas linhas [42] e [45] aplicamos os valores em forma de matriz de n, m, x0 que denotam $\rho_{0}$ nas equações dos capítulos 2 e 3. Desta forma, o programa calcula os coeficientes de forma da onda da equação 3.20 a 3.23 .

\section{(Cálculo dos coeficientes do feixe incidente)}

GTM[n_m_, xo_] $/ ; \mathrm{m}>=0:=\left(4 * \mathrm{Pi}^{*} \mathrm{I}^{\wedge}(\mathrm{n}-\mathrm{m}) *\right.$

$\operatorname{Sqrt}\left[((2 * \mathrm{n}+1) *(\mathrm{n}-\mathrm{m}) !) /\left(\left(4 * \mathrm{Pi}^{*} \mathrm{n} *(\mathrm{n}+1)\right) *(\mathrm{~m}+\mathrm{n}) !\right)\right]^{*}(\mathrm{~m}+\mathrm{n}) ! !^{*}$

$\operatorname{MI} 1[[\mathrm{n}, \mathrm{m}+1, \mathrm{xo}]]) /(\mathrm{n}-\mathrm{m}) !$ !;

GTE $\left[\mathrm{n}_{-}, \mathrm{m}, \mathrm{xo}\right] / ; \mathrm{m}>=0:=\left(4 * \mathrm{Pi}^{*} \mathrm{I}^{\wedge}(-\mathrm{m}+\mathrm{n}+1) * \mathrm{~m} *\right.$

$\operatorname{Sqrt}\left[((2 * n+1) *(n-m) !) /\left(\left(4 * \mathrm{Pi}^{*} \mathrm{n} *(\mathrm{n}+1)\right) *(\mathrm{~m}+\mathrm{n}) !\right)\right] *(\mathrm{~m}+\mathrm{n}) !$ !*

MI2[[n, m + 1, xo]])/(n - m)!!;

GTM $\left[\mathrm{n}_{-}, \mathrm{m}_{-}\right.$, xo_] $/$;

$\mathrm{m}<0:=\overline{(4 *} * \overrightarrow{\mathrm{Pi}^{*}} * \mathrm{I}^{\wedge}(\mathrm{n}-\mathrm{m}) *$

$\operatorname{Sqrt}\left[((2 * n+1) *(m+n) !) /\left(\left(4 * \operatorname{Pi}^{*} n *(n+1)\right) *(n-m) !\right)\right] *(m+n) ! ! *$

$\operatorname{MI} 1[[\mathrm{n}, \operatorname{Abs}[\mathrm{m}]+1, \mathrm{xo}]]) /(\mathrm{n}-\mathrm{m}) !$ !;

GTE $\left[\mathrm{n}_{-}, \mathrm{m}_{-}, \mathrm{xo}\right] / ; \mathrm{m}<0:=\left(4 * \mathrm{Pi}^{*} \mathrm{I}^{\wedge}(-\mathrm{m}+\mathrm{n}+1) * \mathrm{~m}^{*}\right.$

$\operatorname{Sqrt}\left[((2 * n+1) *(m+n) !) /\left(\left(4 * P^{*}{ }^{*}{ }^{*}(n+1)\right) *(n-m) !\right)\right] *(m+n) ! ! *$

$\operatorname{MI} 2[[\mathrm{n}, \operatorname{Abs}[\mathrm{m}]+1, \mathrm{xo}]]) /(\mathrm{n}-\mathrm{m}) !$ !;

GTMp[n_m_, xo_] $/ ; \mathrm{m}>=0:=\left(4 * \mathrm{Pi}^{*} \mathrm{I}^{\wedge}(\mathrm{n}+1) * \mathrm{~m} *\right.$

$\operatorname{Sqrt}\left[((2 * n+1) *(n-m) !) /\left(\left(4 * \operatorname{Pi}^{*} n *(n+1)\right) *(m+n) !\right)\right] *(m+n) ! ! *$

MI2[[n, m + 1, xo]])/(n - m)!!;

GTEp $\left[\mathrm{n}_{-}, \mathrm{m}_{-}, \mathrm{xo}_{-}\right] / ; \mathrm{m}>=0:=-\left(\left(4 * \mathrm{Pi}^{*} \mathrm{I}^{\wedge} \mathrm{n}^{*}\right.\right.$

$\operatorname{Sqrt}\left[((2 * n+1) *(n-m) !) /\left(\left(4 * \operatorname{Pi}^{*} n *(n+1)\right) *(m+n) !\right)\right] *(m+n) ! ! *$

$\operatorname{MI} 1[[\mathrm{n}, \mathrm{m}+1, \mathrm{xo}]]) /(\mathrm{n}-\mathrm{m}) !$ !);

GTMp $\left[\mathrm{n}_{-}, \mathrm{m}_{-}, \mathrm{xo}_{-}\right] / ; \mathrm{m}<0:=\left(4 * \mathrm{Pi}^{*} \mathrm{I}^{\wedge}(\mathrm{n}+1) * \mathrm{~m} *\right.$

$\operatorname{Sqrt}\left[((2 * n+1) *(m+n) !) /\left(\left(4 * P^{*}{ }^{*} n *(n+1)\right) *(n-m) !\right)\right] *(m+n) ! ! *$

$\operatorname{MI} 2[[\mathrm{n}, \operatorname{Abs}[\mathrm{m}]+1, \mathrm{xo}]]) /(\mathrm{n}-\mathrm{m}) !$ !; 
GTEp $\left[\mathrm{n}_{-}, \mathrm{m}_{-}, \mathrm{xo}_{-}\right] / ; \mathrm{m}<0:=-\left(\left(4 * \mathrm{Pi}^{*} \mathrm{I}^{\wedge} \mathrm{n} *\right.\right.$

$\operatorname{Sqrt}\left[((2 * \mathrm{n}+1) *(\mathrm{~m}+\mathrm{n}) !) /\left(\left(4 * \mathrm{Pi}^{*} \mathrm{n} *(\mathrm{n}+1)\right) *(\mathrm{n}-\mathrm{m}) !\right)\right] *(\mathrm{~m}+\mathrm{n}) !$ !*

$\operatorname{MI} 1[[\mathrm{n}, \operatorname{Abs}[\mathrm{m}]+1, \mathrm{xo}]]) /(\mathrm{n}-\mathrm{m}) !$ !;

Em cada linha temos uma equação onde separamos o caso que $\mathrm{m}>0$ do caso em que $\mathrm{m}<0$, e consideramos o valor absoluto para o cálculo, que começa em $\mathrm{m}+1$ e não em $\mathrm{m}$, pois o software inicia a lista em $\mathrm{m}=0$, o que torna a função nula se começa secom $\mathrm{m}$, além disso, tiramos todos os termos que não depende de $\mathrm{m}, \mathrm{n}$ e colocamos na forma final da força. Para $m<0$ usamos a propriedade da integral que faz parte da propriedade da função de Legrande e Bessel em que $\mathrm{m}<0$ torna-se $I(n,-m)=$ $\frac{(n-m) !}{(n+m) !} I(n, m)$, onde I são integrais dos coeficientes, logo, aplicando essa propriedade não é necessário calcular I para $\mathrm{m}<0$,sendo necessária apenas a parte fora da integral que são os coeficientes descritos pelas linhas [47], [48], [52] e [53].

TermosM[n_m_,xo_]:=Block[ $\{$ An,Bn,Cn,Fat1,Fat2 $\}, A n=-$ $2 * \operatorname{At}[[n]] *$ Conjugate $[\operatorname{At}[[n+1]]]+$ Conjugate $[\operatorname{At}[[n+1]]]+\operatorname{At}[[n]]$; $\mathrm{Bn}=-2 * \mathrm{Bt}[[\mathrm{n}]] *$ Conjugate$[\mathrm{Bt}[[\mathrm{n}+1]]]+\operatorname{Conjugate}[\mathrm{Bt}[[\mathrm{n}+1]]]+\mathrm{Bt}[[\mathrm{n}]]$; Fat $1=\operatorname{Re}\left[I^{*}\left(\right.\right.$ Conjugate $\left[B n^{*} \mathrm{GTE}[\mathrm{n},-\mathrm{m}, \mathrm{xo}]\right]^{*} \mathrm{GTE}[\mathrm{n}+1,-$ $(\mathrm{m}+1), \mathrm{xo}]+\mathrm{Bn} *$ Conjugate $[\mathrm{GTE}[\mathrm{n}+1, \mathrm{~m}+1, \mathrm{xo}]] * \mathrm{GTE}[\mathrm{n}, \mathrm{m}, \mathrm{xo}]+$ Conjugate[An*GTM[n,$\mathrm{m}, \mathrm{xo}]]^{*} \mathrm{GTM}[\mathrm{n}+1,-(\mathrm{m}+1), \mathrm{xo}]+\mathrm{An}{ }^{*}$ Conjugate[GTM[n+1,m+1,xo]]*GTM[n,m,xo])];

Fat2 $=\mathrm{If}\left[\mathrm{m}==-\mathrm{n}, 0, \operatorname{Re}\left[\mathrm{I}^{*}(\mathrm{Cn} * \mathrm{GTM}[\mathrm{n}, \mathrm{m}-1, \mathrm{xo}] *\right.\right.$ Conjugate[GTE[n,m,xo]]-

Conjugate[Cn*GTM[n,m,xo] $\left.\left.\left.]^{*} \mathrm{GTE}[\mathrm{n}, \mathrm{m}-1, \mathrm{xo}]\right)\right]\right]$;

$(\operatorname{Sqrt}[(n *(n+2)) /((2 * n+3) *(2 * n+1))] * \operatorname{Sqrt}[(m+n+2) *(m+n+1)] *$ Fat 1$) /(n+1)-(\operatorname{Sqrt}[(-$ $\mathrm{m}+\mathrm{n}+1) *(\mathrm{~m}+\mathrm{n})]^{*}$ Fat 2$\left.) /(\mathrm{n} *(\mathrm{n}+1))\right]$

TermosMp[n,m,xo $]:=\operatorname{Block}[\{$ An,Bn,Cn,Fat1,Fat2 $\}, A n=-$ $2 * \operatorname{At}[[\mathrm{n}]] *$ Conjugate $[\mathrm{At}[[\mathrm{n}+1]]]+$ Conjugate $[\mathrm{At}[[\mathrm{n}+1]]]+\operatorname{At}[[\mathrm{n}]] ; \mathrm{Bn}=-$ $2 * \mathrm{Bt}[[\mathrm{n}]] *$ Conjugate $[\mathrm{Bt}[[\mathrm{n}+1]]]+\operatorname{Conjugate}[\mathrm{Bt}[[\mathrm{n}+1]]]+\mathrm{Bt}[[\mathrm{n}]]$ $\mathrm{Cn}=-2 * \operatorname{At}[[\mathrm{n}]] *$ Conjugate$[\mathrm{Bt}[[\mathrm{n}]]]+$ Conjugate $[\mathrm{Bt}[[\mathrm{n}]]]+\mathrm{At}[[\mathrm{n}]]$

Fat $1=\operatorname{Im}\left[I^{*}\left(\right.\right.$ Conjugate $\left[B n^{*} \mathrm{GTEp}[\mathrm{n},-\mathrm{m}, \mathrm{xo}]\right]^{*} \mathrm{GTEp}[\mathrm{n}+1,-$ $(\mathrm{m}+1), \mathrm{xo}]+\mathrm{Bn} *$ Conjugate[GTEp $[\mathrm{n}+1, \mathrm{~m}+1, \mathrm{xo}]{ }^{*} \mathrm{GTEp}[\mathrm{n}, \mathrm{m}, \mathrm{xo}]+$ Conjugate$\left[\mathrm{An}{ }^{*} \mathrm{GTMp}[\right.$ $\mathrm{n}, \mathrm{m}, \mathrm{xo}]]^{*} \mathrm{GTMp}[\mathrm{n}+1,(\mathrm{~m}+1), \mathrm{xo}]+\mathrm{An} *$ Conjugate $\left.[\mathrm{GTMp}[\mathrm{n}+1, \mathrm{~m}+1, \mathrm{xo}]] * \mathrm{GTMp}[\mathrm{n}, \mathrm{m}, \mathrm{xo}]\right)$ ];

Fat2 $=$ If $\left[m==-n, 0, \operatorname{Im}\left[I^{*}(\mathrm{Cn} * \mathrm{GTMp}[\mathrm{n}, \mathrm{m}-1, \mathrm{xo}] *\right.\right.$ Conjugate[GTEp[n,m,xo]]-

Conjugate[Cn*GTMp[n,m,xo]]*GTEp[n,m-1,xo])]];

$(\operatorname{Sqrt}[(n *(n+2)) /((2 * n+3) *(2 * n+1))] * \operatorname{Sqrt}[(m+n+2) *(m+n+1)] *$ Fat 1$) /(n+1)-(\operatorname{Sqrt}[(-$ $\mathrm{m}+\mathrm{n}+1) *(\mathrm{~m}+\mathrm{n})]^{*}$ Fat 2$\left.) /(\mathrm{n} *(\mathrm{n}+1))\right]$;

SetAttributes[TermosM,Listable];SetAttributes[TermosMp,Listable];

SomaM[n_,xo ]:=Tr[TermosM[n,Range[-n,n],xo]];

SomaMp[n_,xo ]:=Tr[TermosMp[n,Range[-n,n],xo]];

SetAttributes[SomaM,Listable];

SetAttributes[SomaMp,Listable];

ForcaPar[xo_]: $=\left(-\left(\left(0.5^{*} \mathrm{f}\right) / 10^{\wedge} 6\right)^{\wedge} 2\right)^{*} \mathrm{n} 0 \mathrm{n} 1 * \operatorname{Tr}[\operatorname{SomaM}[\operatorname{Range}[\mathrm{Nmax}-1], \mathrm{xo}]]$;

ForcaPerp[xo_]:=((0.5*f)/10^6)^2*n0n $1 * \operatorname{Tr}[$ SomaMp[Range[Nmax-1],xo]];

ForcaParalelo=(ForcaPar[\#1]\&)/@Range[Length[GridX]]; 
dataPar $=$ Transpose $[\{$ GridX,Re[ForcaParalelo $]\}]$;

ForcaPerpendicular $=($ ForcaPerp $[\# 1] \&) / @$ Range $[$ Length[GridX]];

dataPerp $=$ Transpose $[\{$ GridX,Re[ForcaPerpendicular $]\}]$;

TermosZM[n_m_,xo $]:=B \operatorname{lock}[\{$ An,Bn,Cn,Fat1,Fat2,Fat 3$\}, A n=-$

$2 * \operatorname{At}[[\mathrm{n}]]^{*}$ Conjugate $[\operatorname{At}[[\mathrm{n}+1]]]+$ Conjugate $[\operatorname{At}[[\mathrm{n}+1]]]+\operatorname{At}[[\mathrm{n}]]$;

$\mathrm{Bn}=-2 * \mathrm{Bt}[[\mathrm{n}]] *$ Conjugate $[\mathrm{Bt}[[\mathrm{n}+1]]]+$ Conjugate $[\mathrm{Bt}[[\mathrm{n}+1]]]+\mathrm{Bt}[[\mathrm{n}]]$;

$\mathrm{Cn}=-2 * \operatorname{At}[[\mathrm{n}]] *$ Conjugate $[\mathrm{Bt}[[\mathrm{n}]]]+$ Conjugate $[\mathrm{Bt}[[\mathrm{n}]]]+\mathrm{At}[[\mathrm{n}]]$;

Fat $1=\operatorname{Re}\left[I^{*} A n * G T M[n, m, x o] *\right.$ Conjugate $\left.[\mathrm{GTM}[\mathrm{n}+1, \mathrm{~m}, \mathrm{xo}]]\right]$;

Fat2 $=\operatorname{Re}\left[I^{*} \mathrm{Bn} * \mathrm{GTE}[\mathrm{n}, \mathrm{m}, \mathrm{xo}] *\right.$ Conjugate $\left.[\mathrm{GTE}[\mathrm{n}+1, \mathrm{~m}, \mathrm{xo}]]\right] ;$

Fat3 $=\operatorname{Re}\left[I^{*} \mathrm{Cn} * \mathrm{GTM}[\mathrm{n}, \mathrm{m}, \mathrm{xo}] *\right.$ Conjugate[GTE[n,m,xo]]];

$(\operatorname{Sqrt}[(\mathrm{m}+\mathrm{n}+1) *(\mathrm{~m}+\mathrm{n}+1)] * \operatorname{Sqrt}[(\mathrm{n} *(\mathrm{n}+2)) /((2 * \mathrm{n}+3) *(2 * \mathrm{n}+1))] *($ Fat $1+$ Fat 2$)) /(\mathrm{n}+1)+\left(\mathrm{m}^{*}\right.$

Fat3 $) /(n *(n+1))]$;

[75]

TermosZMp[n_,m_,Xo_]:=Block[ $\{$ An,Bn,Cn,Fat1,Fat2,Fat3 $\}, A n=-$

$2 * \operatorname{At}[[\mathrm{n}]]^{*}$ Conjugate[At $\left.[[\mathrm{n}+1]]\right]+$ Conjugate $[\operatorname{At}[[\mathrm{n}+1]]]+\operatorname{At}[[\mathrm{n}]] ;$

$\mathrm{Bn}=-2 * \mathrm{Bt}[[\mathrm{n}]] *$ Conjugate$[\mathrm{Bt}[[\mathrm{n}+1]]]+\operatorname{Conjugate}[\mathrm{Bt}[[\mathrm{n}+1]]]+\mathrm{Bt}[[\mathrm{n}]]$;

$\mathrm{Cn}=-2 * A t[[\mathrm{n}]] *$ Conjugate $[\mathrm{Bt}[[\mathrm{n}]]]+$ Conjugate $[\mathrm{Bt}[[\mathrm{n}]]]+\mathrm{At}[[\mathrm{n}]]$;

Fat $1=\operatorname{Re}\left[I^{*} A n * G T M p[n, m, x o] *\right.$ Conjugate $\left.[\mathrm{GTMp}[\mathrm{n}+1, \mathrm{~m}, \mathrm{xo}]]\right]$;

Fat2 $=\operatorname{Re}\left[I^{*} B n^{*} \mathrm{GTEp}[\mathrm{n}, \mathrm{m}, \mathrm{xo}] *\right.$ Conjugate[GTEp[n+1,m,xo] $]$;

Fat3 $=\operatorname{Re}\left[I^{*} \mathrm{Cn} * \mathrm{GTMp}[\mathrm{n}, \mathrm{m}, \mathrm{xo}]^{*}\right.$ Conjugate[GTEp[n,m,xo]]];

$(\operatorname{Sqrt}[(m+n+1) *(m+n+1)] * \operatorname{Sqrt}[(n *(n+2)) /((2 * n+3) *(2 * n+1))] *($ Fat $1+F a t 2)) /(n+1)+(m *$

Fat 3$) /(n *(n+1))$;

SetAttributes[TermosZM,Listable];SetAttributes[TermosZMp,Listable];

SomaZM[n_,xo_]:=Tr[TermosZM[n,Range[-n,n],xo]];

SomaZMp[n_,xo_]:=Tr[TermosZMp[n,Range[-n,n],xo]];

SetAttributes[SomaZM,Listable];

SetAttributes[SomaZMp,Listable];

ForcaZPar[xo ] : $=0.5^{*}\left(\mathrm{f} / 10^{\wedge} 6\right)^{\wedge} 2 * \mathrm{n} 0 \mathrm{n} 1 * \operatorname{Tr}[$ SomaZM[Range[Nmax-1],xo]];

ForcaZPerp[xo_]: $=0.5^{*}\left(\mathrm{f} / 10^{\wedge} 6\right)^{\wedge} 2 * \mathrm{n} 0 \mathrm{n} 1 * \operatorname{Tr}[$ SomaZMp[Range[Nmax-1],xo]]

ForcaZParalelo=(ForcaZPar[\#1]\&)/@Range[Length[GridX]];

dataZPar $=$ Transpose[ $\{$ GridX,Re[ForcaZParalelo] $\}]$;

ForcaZPerpendicular $=($ ForcaZPerp[\#1]\& $) / @$ Range[Length[GridX]];

dataZPerp=Transpose[ $\{$ GridX,Re[ForcaZPerpendicular] $\}$;

data=Transpose[ \{GridX,ForcaParalelo,ForcaPerpendicular,ForcaZParalelo,ForcaZPerp endicular\}];

ListPlot[dataPar,Joined->False,PlotRange- $>$ All]

Forçatotal $=\operatorname{Sqrt}\left[\left(\operatorname{Re}[\text { ForcaParalelo }]^{\wedge} 2\right)+\left(\operatorname{Re}[\text { ForcaPerpendicular }]^{\wedge} 2\right)+(\operatorname{Re}[\right.$ ForcaZParalel $\left.\left.0]^{\wedge} 2\right)+\left(\operatorname{Re}[\text { ForcaZPerpendicular }]^{\wedge} 2\right)\right]$;

datal $=$ Transpose $[\{$ GridX,Forçatotal $\}]$;

ListPlot[data1,Joined->True,PlotRange->All]

data $2=$ Transpose $[\{$ GridX,Forçatotal $\}]$;

ListPlot[data2,Joined->True,PlotRange- $>$ All]

forçatotal3 $=\mathrm{Re}[$ ForcaParalelo] $+\mathrm{Re}[$ ForcaPerpendicular] $+\mathrm{Re}[$ ForcaZParalelo] $+\mathrm{Re}[$ Forca

ZPerpendicular];

data3 $=$ Transpose $[\{$ GridX,forçatotal 3$\}]$;

ListPlot[data3,Joined-> True,PlotRange->All]

Forçatotal4=Re[ForcaZParalelo];

data4=Transpose[ $\{$ GridX,Forçatotal4 $\}]$; 
ListPlot[data4,Joined->True,PlotRange->All,AxesLabel->

$\left\{" \rho_{0}(\mu \mathrm{m}) "\right.$, "Força em N" $\left.\}\right]$

ListPlot[\{data4,data2 $\}$,Joined->True,PlotRange->All,AxesLabel->\{" $\rho_{0}(\mu \mathrm{m}) "$,"Força em N"\},PlotLegends->\{"Força radial ","Força no eixo x,z"\}] data5=Transpose $[\{$ GridX,ForcaParalelo $\}]$;

ListPlot[data5,Joined-> True,PlotRange- $>$ All,AxesLabel-> $\left\{" \rho_{0}(\mu \mathrm{m}) "\right.$, "Força em N" $\left.\}\right]$

data6=Transpose $[\{$ GridX,ForcaPerpendicular $\}]$

ListPlot[data6,Joined->True,PlotRange->All,AxesLabel->

$\left\{" \rho_{0}(\mu \mathrm{m}) "\right.$, "Força em N"\}]

Forçatotal5=Re[ForcaParalelo]+Re[ForcaZParalelo];

data7=Transpose $[\{$ GridX,Forçatotal5 $\}$;

ListPlot[data7,Joined->True,PlotRange->All,AxesLabel->

$\left\{" \rho_{0}(\mu \mathrm{m}) "\right.$, "Força em N" $\left.\}\right]$

Das linhas [54] a [70] temos construído a parte dos coeficientes da força óptica em x paralela e y perpendicular, que são primeiro os coeficientes de Choque que são as equações 2.134 e 2.135 e depois a força óptica que são as equações 2.132 , sendo que a equação tem seus coeficientes somados nas linhas [61] e [62] e colocado na forma de lista nas linhas [63] e [64]. Depois são adicionadas as constantes para cálculo final da força de acordo com a equação 2.132 nas linhas [65] e [66], onde ela será calculada para todos os valores n, m. As outras variáveis, com exceção de xo, então nas linhas [67]. A linha [70] é calculada para cada xo, que é uma variável continua na teoria, mas que no programa é trata como discreta para evitar logos tempos computacionais. Depois os resultados são colocados em forma de tabela para confeccionar o gráfico.

Da linha [71]a [91] temos o mesmo processo para a equação 3.131, que exerce força, pois não é possível colocar uma força axial nula experimentalmente, e, por fim, da linha [92] a [111] escrevemos somadas as forças em diversas maneiras e construímos os seus gráficos de força em relação a x0 para as quatro forças. 


\section{Apêndice E}

Nesse apêndice apresento o programa para a simulação da força radial com deslocamento focal desenvolvido no software" Mathematica”, versão 11.3, baseado no programa apresentado no apêndice anterior.

Programa para o cálculo da força óptica radial com deslocamento focal

Clear["Global*"];

Off[General::spell];

Off[General::spell1];

SetDirectory[]

$\mathrm{k} 2=\mathrm{k} *(\mathrm{n} 2 / \mathrm{n} 3)$

$\mathrm{z}[\mathrm{x}] \mathrm{e}:=\operatorname{Sqrt}\left[1-\left((\mathrm{n} 3 / \mathrm{n} 2) *\left(1-\mathrm{x}^{\wedge} 2\right)\right)\right]$;

$[6]$ $1=100$;

$\operatorname{tp}[\mathrm{x}]:=(2 * \mathrm{x}) /((\mathrm{n} 2 / \mathrm{n} 3) * \mathrm{x}+\mathrm{z}[\mathrm{x}])$

$\operatorname{ts}[\mathrm{x}]:=(2 * \mathrm{x}) /\left(\mathrm{x}+\mathrm{z}[\mathrm{x}]^{*}(\mathrm{n} 2 / \mathrm{n} 3)\right)$

$\lambda=532 / 1000$;

$\mathrm{NA}=130 / 100$;

$\mathrm{f}=1700$;

$\omega b=1666$

$\mathrm{d}=24 / 10$;

$\mathrm{n} 2=1333 / 1000$;

$\mathrm{n} 3=\operatorname{Sqrt}\left[(103961212 / 100000000)^{*} \lambda^{\wedge} 2 /\left(\lambda^{\wedge} 2-\right.\right.$

$(600069867 / 100000000000))+(231792344 / 1000000000) * \lambda^{\wedge} 2 /(\lambda \wedge 2-$

$(200179144 / 10000000000))+(101046945 / 100000000) * \lambda^{\wedge} 2 /\left(\lambda^{\wedge} 2-(103560653 / 1000000)\right.$

$+1]$;

$\mathrm{n} 1=\operatorname{Sqrt}\left[1+(14435 / 10000) * \lambda^{\wedge} 2 /\left(\lambda^{\wedge} 2-(20216 / 1000000)\right)\right]$;

$\mathrm{k}=(2 * \mathrm{Pi} * \mathrm{n} 2) / \lambda$;

n0n $1=\operatorname{Sqrt}[1 / \mathrm{n} 3]$

$\alpha \max =\operatorname{ArcSin}[\mathrm{NA} / \mathrm{n} 2]$;

$\mathrm{kf}=\mathrm{k} * \mathrm{f}$;

$\mathrm{f} \omega=(\mathrm{f} / \omega \mathrm{b}) * 0.1$

fo $=1 /(f \omega * \operatorname{Sin}[\alpha \max ])$;

$\eta=\mathrm{n} 1 / \mathrm{n} 2$;

$\chi=\mathrm{Pi}^{*} \mathrm{~d} *(\mathrm{n} 2 / \lambda)$

$\mathrm{P}=$ Table $[\mathrm{P},\{\mathrm{P}, \mathrm{Pmin}, \mathrm{Pmax}\}]$;

$\mathrm{eE} 2=(2 * \mathrm{P} *(0.6)) /(\mathrm{n} 2 * \mathrm{c} *$

$\left.\mathrm{Pi}^{*}\left(\backslash[\mathrm{Omega}]^{\wedge} 2\right)\right)^{*} 10^{\wedge}(-5)$

Nmax $=$ IntegerPart $[\chi]+2$;

$\mathrm{Xini}=1 / 100 ; \mathrm{Xinc}=1 / 100 ; \mathrm{Xfin}=453 / 100 ; \mathrm{X}=1$; 
GridXN=Table $[\{\mathrm{n}, \mathrm{m}, \mathrm{xx}\},\{\mathrm{n}, 1, \mathrm{Nmax}+1\},\{\mathrm{m}, 0, \mathrm{n}\},\{\mathrm{xx}, \mathrm{Xini}, \mathrm{Xfin}, \mathrm{Xinc}\}]$;

Xini=First[GridX];Xfin=Last[GridX];

(Cálculo dos coeficientes de espalhamento ou coeficientes de Mie)

$\psi\left[\mathrm{n}_{2}, \mathrm{y}\right]=\mathrm{y} *$ SphericalBesselJ $[\mathrm{n}, \mathrm{y}]$;

$\zeta\left[\mathrm{n}_{-}, \mathrm{y}_{-}\right]=\mathrm{y} *$ SphericalHankelH1[n,y];

$\mathrm{D} \psi\left[\mathrm{n}_{-}, \mathrm{y}\right]=\mathrm{D}[\psi[\mathrm{n}, \mathrm{y}], \mathrm{y}]$;

$\mathrm{D} \zeta\left[\mathrm{n}_{-}, \mathrm{y}\right]=\mathrm{D}[\zeta[\mathrm{n}, \mathrm{y}], \mathrm{y}]$;

$\mathrm{AB}[\mathrm{n}, \mathrm{xx}, \mathrm{mm}]=\operatorname{Module}[\{\mathrm{vPsi}, \mathrm{vPsiM}, \mathrm{vZeta}, \mathrm{vDPsi}, \mathrm{vDPsiM}, \mathrm{vDZeta}\}, \mathrm{vPsi}=\psi[\mathrm{n}, \mathrm{xx}] ; \mathrm{v}$

PsiM $=\psi\left[n, m m^{*} x x\right]$;

$\mathrm{vZeta}=\zeta[\mathrm{n}, \mathrm{xx}]$;

$\mathrm{vDPsi}=\mathrm{D} \psi[\mathrm{n}, \mathrm{xx}] ; \mathrm{vDPsiM}=\mathrm{D} \psi[\mathrm{n}, \mathrm{mm} * \mathrm{xx}] ; \mathrm{vDZeta}=\mathrm{D} \zeta[\mathrm{n}, \mathrm{xx}]$

$\left\{\left(\mathrm{mm}^{*}{ }_{\mathrm{vPsiM}}{ }^{*} \mathrm{vDPsi}-\mathrm{vPsi}{ }^{*} \mathrm{vDPsiM}\right) /\left(\mathrm{mm}^{*} \mathrm{vPsiM}{ }^{*} \mathrm{vDZeta}-\right.\right.$

vZeta*vDPsiM),(vPsiM*vDPsi-mm*vPsi*vDPsiM)/(vPsiM*vDZeta-

mm*vZeta*vDPsiM) $\}$ ];

$\{\mathrm{At}, \mathrm{Bt}\}=\mathrm{AB}[\mathrm{GridN}, \chi, \eta]$

$\mathrm{I} 12\left[\mathrm{n}_{-}, \mathrm{m}_{-}, \mathrm{xo}\right] \mathrm{e}:=$

Block $[\{$ func $\}$,

func $\left[\left(\mathrm{x}_{-}\right)\right.$?NumericQ] :=

$\operatorname{Sqrt}[\mathrm{x}] *\left(\left(\left(\mathrm{~m}^{\wedge} 2\right)^{*}\right.\right.$

LegendreP[n, $\mathrm{m}$,

$\mathrm{z}[\mathrm{x}]]^{*}($ BesselJ $[\mathrm{m}$,

$\left.\left.\mathrm{k}^{*} \mathrm{xo} * \operatorname{Sqrt}\left[1-\mathrm{x}^{\wedge} 2\right]\right] /\left(\mathrm{k}^{*} \mathrm{xo} *\left(1-\mathrm{x}^{\wedge} 2\right)\right)\right) *(\mathrm{ts}[\mathrm{x}]-$

$\left.\mathrm{z}[\mathrm{x}]^{*} \mathrm{tp}[\mathrm{x}]\right)+\left(\mathrm{m}^{*} \mathrm{z}[\mathrm{x}] * \text { LegendreP[n, } \mathrm{m}, \mathrm{z}[\mathrm{x}]\right]^{*}$

BesselJ $\left.\left.\left[\mathrm{m}+1, \mathrm{k}^{*} \mathrm{xo} \mathrm{o}^{*} \operatorname{Sqrt}\left[1-\mathrm{x}^{\wedge} 2\right]\right]^{*} \operatorname{tp}[\mathrm{x}]\right)\right)-(\mathrm{m}+\mathrm{n}) *(-\mathrm{m}+$

$\mathrm{n}+1)^{*} \operatorname{tp}[\mathrm{x}]^{*}\left(\operatorname{Sqrt}\left[1-\mathrm{x}^{\wedge} 2\right]\right)^{*}$

LegendreP[n, $\mathrm{m}-1$,

$\mathrm{z}[\mathrm{x}]]^{*}\left(\mathrm{~m}^{*}\right.$

$\operatorname{Besse} 1 \mathrm{~J}\left[\mathrm{~m}, \mathrm{k}^{*} \mathrm{xo}^{*} \operatorname{Sqrt}\left[1-\mathrm{x}^{\wedge} 2\right]\right] /\left(\mathrm{k}^{*} \mathrm{xo}^{*} \operatorname{Sqrt}\left[1-\mathrm{x}^{\wedge} 2\right]\right)-$

BesselJ $[\mathrm{m}+1$,

$\left.\left.\left.\mathrm{k}^{*} \mathrm{xo}^{*} \operatorname{Sqrt}\left[1-\mathrm{x}^{\wedge} 2\right]\right]\right)\right) /\left(\mathrm{E}^{\wedge}\left(\mathrm{f}[\mathrm{O} \text { Omega }]^{\wedge} 2 *\left(1-\mathrm{x}^{\wedge} 2\right)\right)^{*}\right.$

$\left.\mathrm{E}^{\wedge}\left(-\left(\mathrm{I}^{*} \mathrm{k} 2 * 1^{*} \mathrm{z}[\mathrm{x}]\right)\right)^{*} \mathrm{E}^{\wedge}\left(\mathrm{I}^{*} \mathrm{k}^{*} 1^{*} \mathrm{x}\right)\right)$;

NIntegrate $[((\mathrm{n}-\mathrm{m}) !$ !*func $[\mathrm{x}]) /(\mathrm{m}+\mathrm{n}) !$ !, $\{\mathrm{x}, \operatorname{cosmax}, 1\}$,

AccuracyGoal -> 1, Compiled $->$ False, MaxRecursion $->$ 30,

WorkingPrecision $->30$,

Method -> \{"OscillatorySelection", "SymbolicProcessing" ->0\}]];

MI1 = Parallelize[Apply[I12[\#1, \#2, \#3] \&, GridXN, \{3\}]];

$\mathrm{I} 22\left[\mathrm{n}_{-}, \mathrm{m}_{-}, \mathrm{xo}\right] \mathrm{:}:=$

Block $[\{$ func $\}$,

func $\left[\left(\mathrm{x}_{-}\right)\right.$?NumericQ $]:=$

Sqrt $[\mathrm{x}]^{*}\left(\left(\right.\right.$ LegendreP $[\mathrm{n}, \mathrm{m}, \mathrm{z}[\mathrm{x}]]^{*}$

$\operatorname{ts}[\mathrm{x}])^{*}\left(\mathrm{~m} * \operatorname{BesselJ}\left[\mathrm{m}, \mathrm{k}^{*} \mathrm{xo} \mathrm{O}^{*} \operatorname{Sqrt}\left[1-\mathrm{x}^{\wedge} 2\right]\right]\right) /\left(\mathrm{k}^{*} \mathrm{xo}^{*}\right.$

Sqrt $\left.\left.\left[1-x^{\wedge} 2\right]\right)-(\text { LegendreP[n, m, z }[x]]^{*} \operatorname{ts}[x]\right)^{*}$

BesselJ $[\mathrm{m}+1$,

$$
\left.\mathrm{k}^{*} \mathrm{xo}^{*} \operatorname{Sqrt}\left[1-\mathrm{x}^{\wedge} 2\right]\right]-\left((\mathrm{m}+\mathrm{n}) *(-\mathrm{m}+\mathrm{n}+1) * \operatorname{tp}[\mathrm{x}]^{*}\right.
$$

Sqrt $\left[1-\mathrm{x}^{\wedge} 2\right]^{*}$ LegendreP[n, $\left.\mathrm{m}-1, \mathrm{z}[\mathrm{x}]\right]^{*}$

BesselJ[m, k*xo*Sqrt[1 - $\left.\left.\left.\mathrm{x}^{\wedge} 2\right]\right]\right) /\left(\mathrm{k}^{*} \mathrm{xo}^{*} \operatorname{Sqrt}\left[1-\mathrm{x}^{\wedge} 2\right]\right)-\left(\mathrm{m}^{*}\right.$

$\operatorname{tp}[\mathrm{x}]^{*}$ LegendreP $[\mathrm{n}, \mathrm{m}, \mathrm{z}[\mathrm{x}]]^{*} \mathrm{z}[\mathrm{x}]^{*}$

$\left.\operatorname{BesselJ}\left[\mathrm{m}, \mathrm{k}^{*} \mathrm{xo}{ }^{*} \operatorname{Sqrt}\left[1-\mathrm{x}^{\wedge} 2\right]\right]\right) /\left(\mathrm{k}^{*}\right.$ 
$\left.\mathrm{E}^{\wedge}\left(\mathrm{I}^{*} \mathrm{k} * \mathrm{I}^{*} \mathrm{x}\right)\right)$

$\left.\left.\mathrm{xo}^{*}\left(1-\mathrm{x}^{\wedge} 2\right)\right)\right) /\left(\mathrm{E}^{\wedge}\left(\mathrm{f} \backslash[\mathrm{Omega}]^{\wedge} 2 *\left(1-\mathrm{x}^{\wedge} 2\right)\right)^{*} \mathrm{E}^{\wedge}\left(-\mathrm{I}^{*} \mathrm{k} 2 * 1 * \mathrm{z}[\mathrm{x}]\right)^{*}\right.$

NIntegrate $\left[\left((\mathrm{n}-\mathrm{m}) ! !^{*}\right.\right.$ func $\left.[\mathrm{x}]\right) /(\mathrm{m}+\mathrm{n}) !$ !, $\{\mathrm{x}, \operatorname{cosmax}, 1\}$,

AccuracyGoal $->1$, Compiled $->$ False, MaxRecursion $->$ 30,

WorkingPrecision $->30$,

Method -> \{"OscillatorySelection", "SymbolicProcessing" -> 0 $\}]$;

MI2 = Parallelize[Apply[I22[\#1, \#2, \#3] \&, GridXN, \{3\}]];

A diferença desse programa se dá nas novas formas das integrais que levam em conta o deslocamento focal, apresentadas nas equações 3.24 e 3.25, através da adição de novas variáveis: na linha [7] o deslocamento do foco, em mícrons; nas linhas [8] e [9], as funções tp e ts; e na linha [6] a nova variável integrável z[x]; além de que, na linha [5], temos um novo k2, que depende de k. Desta forma, essa variáveis são escritas como $d, t^{p}, t^{s}, x_{2}=\cos \left[\alpha_{2}\right]$ e $k_{2}$, como descrito nas equações do capítulo 3. Essas são as únicas diferenças em relação ao programa sem deslocamento focal, além dos parâmetros de precisão de máquina e a máxima recursão que são menores, pois a equação ficaria muito mais trabalhosa para o computador, demorando muito mais tempo para calcular.

\section{(Os coeficientes do feixe incidente com fenômeno de deslocamento focal)}

GTM[n_m_,xo_] $/ ; \mathrm{m}>=0:=\left(4 * \mathrm{Pi}^{*} \mathrm{I}^{\wedge}(\mathrm{n}-\mathrm{m}) * \operatorname{Sqrt}[((2 * \mathrm{n}+1) *(\mathrm{n}-\right.$ $\left.\mathrm{m}) !) /\left(\left(4 * \mathrm{Pi}^{*} \mathrm{n}^{*}(\mathrm{n}+1)\right) *(\mathrm{~m}+\mathrm{n}) !\right)\right]^{*}(\mathrm{~m}+\mathrm{n}) !$ ! $\left.\left.{ }^{*} \mathrm{MI} 1[\mathrm{n}, \mathrm{m}+1, \mathrm{xo}]\right]\right) /(\mathrm{n}-\mathrm{m}) !$ !;

GTE[n_m_,xo $] / ; m>=0:=\left(4 * \operatorname{Pi}^{*} I^{\wedge}(-m+n+1) * m * \operatorname{Sqrt}[((2 * n+1) *(n-\right.$

$\left.\left.\mathrm{m}) !) /\left(\left(4 * \mathrm{Pi}{ }^{*} \mathrm{n} *(\mathrm{n}+1)\right) *(\mathrm{~m}+\mathrm{n}) !\right)\right] *(\mathrm{~m}+\mathrm{n}) ! ! * \operatorname{MI} 2[[\mathrm{n}, \mathrm{m}+1, \mathrm{xo}]]\right) /(\mathrm{n}-\mathrm{m}) ! !$

GTM $[\mathrm{n}, \mathrm{m}, \mathrm{xo}] / ; \mathrm{m}<0:=\left(4 * \mathrm{Pi}^{*} \mathrm{I}^{\wedge}(\mathrm{n}-\right.$

$\mathrm{m}) * \operatorname{Sqrt}\left[((2 * \mathrm{n}+1) *(\mathrm{~m}+\mathrm{n}) !) /\left(\left(4 * \mathrm{Pi}^{*} \mathrm{n} *(\mathrm{n}+1)\right) *(\mathrm{n}-\right.\right.$

$\left.\mathrm{m}) !)]^{*}(\mathrm{~m}+\mathrm{n}) ! !^{*} \operatorname{MI} 1[[\mathrm{n}, \mathrm{Abs}[\mathrm{m}]+1, \mathrm{xo}]]\right) /(\mathrm{n}-\mathrm{m}) !$ !;

GTE $[\mathrm{n}, \mathrm{m}, \mathrm{xo}] / ; \mathrm{m}<0:=\left(4 * \mathrm{Pi}^{*} \mathrm{I}^{\wedge}(-\right.$

$\mathrm{m}+\mathrm{n}+1) * \mathrm{~m} * \operatorname{Sqrt}\left[((2 * \mathrm{n}+1) *(\mathrm{~m}+\mathrm{n}) !) /\left(\left(4 * \mathrm{Pi}^{*} \mathrm{n} *(\mathrm{n}+1)\right) *(\mathrm{n}-\right.\right.$

$\left.\mathrm{m}) !)]^{*}(\mathrm{~m}+\mathrm{n}) ! !^{*} \mathrm{MI} 2[[\mathrm{n}, \mathrm{Abs}[\mathrm{m}]+1, \mathrm{xo}]]\right) /(\mathrm{n}-\mathrm{m}) !$ !;

GTMp[n_m_,xo_] $/ ; \mathrm{m}>=0:=\left(4 * \operatorname{Pi}^{*} \mathrm{I}^{\wedge}(\mathrm{n}+1) * \mathrm{~m} * \operatorname{Sqrt}[((2 * \mathrm{n}+1) *(\mathrm{n}-\right.$

$\left.\mathrm{m}) !) /\left(\left(4 * \mathrm{Pi}^{*}{ }^{*} *(\mathrm{n}+1)\right) *(\mathrm{~m}+\mathrm{n}) !\right)\right] *(\mathrm{~m}+\mathrm{n}) !$ !*MI2[[n,m+1,xo]])/(n-m)!!;

GTEp[n_,m_,xo $] / ; m>=0:=-\left(\left(4 * \operatorname{Pi}^{*} \mathrm{I}^{\wedge} \mathrm{n} * \operatorname{Sqrt}[((2 * \mathrm{n}+1) *(\mathrm{n}-\right.\right.$

$\left.\mathrm{m}) !) /\left(\left(4 * \mathrm{Pi}^{*} \mathrm{n}^{*}(\mathrm{n}+1)\right) *(\mathrm{~m}+\mathrm{n}) !\right)\right] *(\mathrm{~m}+\mathrm{n}) !$ ! $\left.\left.* \operatorname{MI} 1[[\mathrm{n}, \mathrm{m}+1, \mathrm{xo}]]\right) /(\mathrm{n}-\mathrm{m}) ! !\right) ; \quad[50]$

GTMp[n_m_,Xo_] $/ ; \mathrm{m}<0:=\left(4 * \mathrm{Pi}^{*} \mathrm{I}^{\wedge}(\mathrm{n}+1) * \mathrm{~m} * \operatorname{Sqrt}\left[((2 * \mathrm{n}+1) *(\mathrm{~m}+\mathrm{n}) !) /\left(\left(4 * \mathrm{Pi}^{*} \mathrm{n}^{*}(\right.\right.\right.\right.$

$\left.\left.\mathrm{n}+1))^{*}(\mathrm{n}-\mathrm{m}) !\right)\right]^{*}(\mathrm{~m}+\mathrm{n}) !$ !*MI2[[n,Abs[m]+1,xo]])/(n-m)!!;

GTEp[n_m_,xo $] / ; \mathrm{m}<0:=-$

$\left(\left(4 * \mathrm{Pi}^{*} \mathrm{I}^{\wedge} \mathrm{n} * \operatorname{Sqrt}\left[((2 * \mathrm{n}+1) *(\mathrm{~m}+\mathrm{n}) !) /\left(\left(4 * \mathrm{Pi}^{*} \mathrm{n} *(\mathrm{n}+1)\right) *(\mathrm{n}-\right.\right.\right.\right.$

$\mathrm{m}) !)]^{*}(\mathrm{~m}+\mathrm{n}) !$ !*MI1[[n,Abs[m]+1,xo]])/(n-m)!!); 
TermosM[n_m_,xo_]:=Block $[\{\mathrm{An}, \mathrm{Bn}, \mathrm{Cn}$, Fat 1, Fat 2$\}, \mathrm{An}=-$

$2 * \operatorname{At}[[\mathrm{n}]] *$ Conjugate[At $[[\mathrm{n}+1]]]+$ Conjugate$[\operatorname{At}[[\mathrm{n}+1]]]+\operatorname{At}[[\mathrm{n}]] ;$

$\mathrm{Bn}=-2 * \mathrm{Bt}[[\mathrm{n}]] *$ Conjugate$[\mathrm{Bt}[[\mathrm{n}+1]]]+$ Conjugate $[\mathrm{Bt}[[\mathrm{n}+1]]]+\mathrm{Bt}[[\mathrm{n}]]$;

$\mathrm{Cn}=-2 * A t[[\mathrm{n}]] *$ Conjugate $[\mathrm{Bt}[[\mathrm{n}]]]+$ Conjugate $[\mathrm{Bt}[[\mathrm{n}]]]+\mathrm{At}[[\mathrm{n}]]$;

Fat1 $=\operatorname{Re}\left[I^{*}\left(\right.\right.$ Conjugate $\left[B n^{*} \mathrm{GTE}[\mathrm{n},-\mathrm{m}, \mathrm{xo}]\right]^{*} \mathrm{GTE}[\mathrm{n}+1,-$

$(\mathrm{m}+1), \mathrm{xo}]+\mathrm{Bn} *$ Conjugate $[\mathrm{GTE}[\mathrm{n}+1, \mathrm{~m}+1, \mathrm{xo}]] * \mathrm{GTE}[\mathrm{n}, \mathrm{m}, \mathrm{xo}]+$ Conjugate$[A n * G T M[n,-$ $\mathrm{m}, \mathrm{xo}]]^{*} \mathrm{GTM}[\mathrm{n}+1,-(\mathrm{m}+1), \mathrm{xo}]+\mathrm{An}{ }^{*}$ Conjugate[GTM $\left.\left.\left.[\mathrm{n}+1, \mathrm{~m}+1, \mathrm{xo}]\right] * \mathrm{GTM}[\mathrm{n}, \mathrm{m}, \mathrm{xo}]\right)\right]$;

Fat2 $=\mathrm{If}\left[\mathrm{m}==-\mathrm{n}, 0, \operatorname{Re}\left[\mathrm{I}^{*}\left(\mathrm{Cn}{ }^{*} \mathrm{GTM}[\mathrm{n}, \mathrm{m}-1, \mathrm{xo}] *\right.\right.\right.$ Conjugate[GTE[n,m,xo]]-

Conjugate $\left.\left.\left.[\mathrm{Cn} * \mathrm{GTM}[\mathrm{n}, \mathrm{m}, \mathrm{xo}]]^{*} \mathrm{GTE}[\mathrm{n}, \mathrm{m}-1, \mathrm{xo}]\right)\right]\right]$;

$(\operatorname{Sqrt}[(n *(n+2)) /((2 * n+3) *(2 * n+1))] * \operatorname{Sqrt}[(m+n+2) *(m+n+1)] *$ Fat 1$) /(n+1)-(\operatorname{Sqrt}[(-$

$\mathrm{m}+\mathrm{n}+1) *(\mathrm{~m}+\mathrm{n})]^{*}$ Fat 2$\left.) /(\mathrm{n} *(\mathrm{n}+1))\right]$;

TermosMp[n_m_,Xo_]:=Block[ $\{\mathrm{An}, \mathrm{Bn}, \mathrm{Cn}, \mathrm{Fat} 1, \mathrm{Fat} 2\}, \mathrm{An}=$

$2 * \operatorname{At}[[\mathrm{n}]] *$ Conjugate $[\mathrm{At}[[\mathrm{n}+1]]]+$ Conjugate $[\mathrm{At}[[\mathrm{n}+1]]]+\mathrm{At}[[\mathrm{n}]] ; \mathrm{Bn}=-$

$2 * \mathrm{Bt}[[\mathrm{n}]] *$ Conjugate $[\mathrm{Bt}[[\mathrm{n}+1]]]+$ Conjugate $[\mathrm{Bt}[[\mathrm{n}+1]]]+\mathrm{Bt}[[\mathrm{n}]]$

$\mathrm{Cn}=-2 * A t[[\mathrm{n}]] *$ Conjugate $[\mathrm{Bt}[[\mathrm{n}]]]+$ Conjugate $[\mathrm{Bt}[[\mathrm{n}]]]+\mathrm{At}[[\mathrm{n}]]$;

Fat $1=\operatorname{Im}\left[I^{*}\left(\right.\right.$ Conjugate $[\mathrm{Bn} * \mathrm{GTEp}[\mathrm{n},-\mathrm{m}, \mathrm{xo}]]^{*} \mathrm{GTEp}[\mathrm{n}+1,-$

$(\mathrm{m}+1), \mathrm{xo}]+\mathrm{Bn} *$ Conjugate[GTEp[n+1,m+1,xo]]*GTEp[n,m,xo]+Conjugate[An*GTMp[

$\mathrm{n},-\mathrm{m}, \mathrm{xo}]]^{*} \mathrm{GTMp}[\mathrm{n}+1,-$

$(\mathrm{m}+1), \mathrm{xo}]+\mathrm{An} *$ Conjugate $\left.\left.[\mathrm{GTMp}[\mathrm{n}+1, \mathrm{~m}+1, \mathrm{xo}]]^{*} \mathrm{GTMp}[\mathrm{n}, \mathrm{m}, \mathrm{xo}]\right)\right]$;

Fat2 $=$ If $\left[m==-n, 0, \operatorname{Im}\left[I^{*}\left(\mathrm{Cn} * \mathrm{GTMp}[\mathrm{n}, \mathrm{m}-1, \mathrm{xo}]^{*}\right.\right.\right.$ Conjugate[GTEp[n,m,xo]]-

Conjugate[Cn*GTMp[n,m,xo]]*GTEp[n,m-1,xo])]];

$(\operatorname{Sqrt}[(\mathrm{n} *(\mathrm{n}+2)) /((2 * \mathrm{n}+3) *(2 * \mathrm{n}+1))] * \operatorname{Sqrt}[(\mathrm{m}+\mathrm{n}+2) *(\mathrm{~m}+\mathrm{n}+1)] *$ Fat 1$) /(\mathrm{n}+1)-(\operatorname{Sqrt}[(-$

$\mathrm{m}+\mathrm{n}+1) *(\mathrm{~m}+\mathrm{n})]^{*}$ Fat 2$\left.) /(\mathrm{n} *(\mathrm{n}+1))\right]$;

SetAttributes[TermosM,Listable];SetAttributes[TermosMp,Listable];

SomaM[n_,xo ]: $=\operatorname{Tr}[$ TermosM[n,Range[-n,n],xo]];

SomaMp[n_,xo ]:=Tr[TermosMp[n,Range[-n,n],xo]];

SetAttributes[SomaM,Listable];

SetAttributes[SomaMp,Listable];

ForcaPar[xo_]:=eE2*(-(((1/2)*f)/10^6)^2)*n0n1*Tr[SomaM[Range[Nmax-1],xo]];

ForcaPerp[xo_]:=eE2*(((1/2)*f)/10^6)^2*n0n1*Tr[SomaMp[Range[Nmax-1],xo]];

ForcaParalelo=(ForcaPar[\#1]\&)/@Range[Length[GridX]];

dataPar $=$ Transpose[ $\{$ GridX, Re[ForcaParalelo] $\}$;

ForcaPerpendicular $=($ ForcaPerp[\#1]\& $) / @$ Range[Length[GridX]]

dataPerp=Transpose[ $\{$ GridX,Re[ForcaPerpendicular $]\}]$

TermosZM[n_m_,xo_]:=Block[ $\{\mathrm{An}, \mathrm{Bn}, \mathrm{Cn}$, Fat1,Fat2,Fat3 $\}, \mathrm{An}==$ $2 * \operatorname{At}[[n]] *$ Conjugate $[\operatorname{At}[[n+1]]]+$ Conjugate $[\operatorname{At}[[n+1]]]+\operatorname{At}[[n]] ;$

$\mathrm{Bn}=-2 * \mathrm{Bt}[[\mathrm{n}]] *$ Conjugate $[\mathrm{Bt}[[\mathrm{n}+1]]]+$ Conjugate $[\mathrm{Bt}[[\mathrm{n}+1]]]+\mathrm{Bt}[[\mathrm{n}]]$;

$\mathrm{Cn}=-2 * \mathrm{At}[[\mathrm{n}]] *$ Conjugate $[\mathrm{Bt}[[\mathrm{n}]]]+$ Conjugate$[\mathrm{Bt}[[\mathrm{n}]]]+\mathrm{At}[[\mathrm{n}]] ;$

Fat1 $=\operatorname{Re}\left[I^{*} A n^{*} \mathrm{GTM}[\mathrm{n}, \mathrm{m}, \mathrm{xo}]^{*}\right.$ Conjugate[GTM[n+1,m,xo]]]; 
Fat2 $=\operatorname{Re}\left[I^{*} \mathrm{Bn} * \mathrm{GTE}[\mathrm{n}, \mathrm{m}, \mathrm{xo}] *\right.$ Conjugate $\left.[\mathrm{GTE}[\mathrm{n}+1, \mathrm{~m}, \mathrm{xo}]]\right] ;$

Fat3 $=\operatorname{Re}\left[I^{*} \mathrm{Cn} * \mathrm{GTM}[\mathrm{n}, \mathrm{m}, \mathrm{xo}] *\right.$ Conjugate $\left.[\mathrm{GTE}[\mathrm{n}, \mathrm{m}, \mathrm{xo}]]\right]$;

$(\operatorname{Sqrt}[(\mathrm{m}+\mathrm{n}+1) *(-$

$\mathrm{m}+\mathrm{n}+1)] * \operatorname{Sqrt}[(\mathrm{n} *(\mathrm{n}+2)) /((2 * \mathrm{n}+3) *(2 * \mathrm{n}+1))] *($ Fat1 + Fat 2$)) /(\mathrm{n}+1)+\left(\mathrm{m}^{*}\right.$ Fat 3$\left.) /(\mathrm{n} *(\mathrm{n}+1))\right]$;

[66]

TermosZMp[n_m_,xo_]:=Block[ $\{$ An,Bn,Cn,Fat1,Fat2,Fat3 $\}, A n=-$

$2 * \operatorname{At}[[n]] *$ Conjugate $[\operatorname{At}[[n+1]]]+$ Conjugate[At $[[n+1]]]+\operatorname{At}[[n]] ;$

$\mathrm{Bn}=-2 * \mathrm{Bt}[[\mathrm{n}]] *$ Conjugate $[\mathrm{Bt}[[\mathrm{n}+1]]]+\operatorname{Conjugate}[\mathrm{Bt}[[\mathrm{n}+1]]]+\mathrm{Bt}[[\mathrm{n}]]$;

$\mathrm{Cn}=-2 * \mathrm{At}[[\mathrm{n}]]^{*}$ Conjugate $[\mathrm{Bt}[[\mathrm{n}]]]+$ Conjugate $[\mathrm{Bt}[[\mathrm{n}]]]+\mathrm{At}[[\mathrm{n}]]$;

Fat $1=\operatorname{Re}\left[I^{*} A n * G T M p[n, m, x o] *\right.$ Conjugate $\left.[\mathrm{GTMp}[\mathrm{n}+1, \mathrm{~m}, \mathrm{xo}]]\right]$;

Fat2 $=\operatorname{Re}\left[I^{*} B n * G T E p[n, m, x o] *\right.$ Conjugate[GTEp[n+1,m,xo]]];

Fat3 $=\operatorname{Re}\left[I^{*} \mathrm{Cn} * \mathrm{GTMp}[\mathrm{n}, \mathrm{m}, \mathrm{xo}] *\right.$ Conjugate[GTEp[n,m,xo]]];

$(\operatorname{Sqrt}[(\mathrm{m}+\mathrm{n}+1) *(-$

$m+n+1)] * \operatorname{Sqrt}[(n *(n+2)) /((2 * n+3) *(2 * n+1))] *($ Fat $1+$ Fat 2$)) /(n+1)+(m *$ Fat 3$) /(n *(n+1))] ;$

SetAttributes[TermosZM,Listable];SetAttributes[TermosZMp,Listable];

SomaZM[n_,xo_]:=Tr[TermosZM[n,Range[-n,n],xo]];

SomaZMp[n_,X_]:=Tr[TermosZMp[n,Range[-n,n],xo]];

SetAttributes[SomaZM,Listable];

SetAttributes[SomaZMp,Listable;

ForcaZPar[xo ] : = eE2* $(1 / 2)^{*}\left(\mathrm{f} / 10^{\wedge} 6\right)^{\wedge} 2 * \mathrm{n} 0 \mathrm{n} 1 * \operatorname{Tr}[\operatorname{SomaZM}[\operatorname{Range}[\mathrm{Nmax}-1], \mathrm{xo}]]$;

ForcaZPerp[xo_]:= eE2* $(1 / 2)^{*}\left(\mathrm{f} / 10^{\wedge} 6\right)^{\wedge} 2 * \mathrm{n} 0 \mathrm{n} 1 * \operatorname{Tr}[\operatorname{SomaZMp}[\operatorname{Range}[\mathrm{Nmax}-1], \mathrm{xo}]]$;

ForcaZParalelo=(ForcaZPar[\#1]\&)/@Range[Length[GridX]];

dataZPar $=$ Transpose $[\{$ GridX,Re[ForcaZParalelo $]\}]$;

ForcaZPerpendicular $=($ ForcaZPerp $[\# 1] \&) / @$ Range $[$ Length[GridX]];

dataZPerp=Transpose[ $\{$ GridX,Re[ForcaZPerpendicular $]\}$;

data $=$ Transpose $[\{$ GridX,ForcaParalelo,ForcaPerpendicular,ForcaZParalelo,ForcaZPerp endicular\}];

Forçatotal $=\operatorname{Sqrt}\left[\left(\operatorname{Re}[\text { ForcaZParalelo }]^{\wedge} 2\right)+\left(\operatorname{Re}[\text { ForcaZPerpendicular }]^{\wedge} 2\right)+(\operatorname{Re}[\right.$ ForcaParal elo $\left.\left.]^{\wedge} 2\right)+\left(\operatorname{Re}[\text { ForcaPerpendicular }]^{\wedge} 2\right)\right]$;

data $1=$ Transpose $[\{$ GridX,Forçatotal $\}]$;

ListPlot[data1,Joined-> True,PlotRange- $>$ All]

forçatotal3 $=\mathrm{Re}[$ ForcaParalelo] $+\mathrm{Re}[$ ForcaPerpendicular] $+\mathrm{Re}[$ ForcaZParalelo] $+\mathrm{Re}[$ Forca ZPerpendicular];

data3 $=$ Transpose $[\{$ GridX,forçatotal3 $\}]$;

ListPlot[data3,Joined-> True,PlotRange- $>$ All]

Forçatotal $2=\operatorname{Sqrt}\left[\left(\operatorname{Re}[\text { ForcaParalelo }]^{\wedge} 2\right)+\left(\operatorname{Re}[\text { ForcaPerpendicular }]^{\wedge} 2\right)\right.$

data2 $=$ Transpose $[\{$ GridX,Forçatotal2 $\}]$; 
Forçatotal4=Re[ForcaZParalelo];

data4=Transpose $[\{$ GridX,Forçatotal4 $\}]$;

ListPlot[data4,Joined-> True,PlotRange->All,AxesLabel->

$\left\{" \rho_{0}(\mu \mathrm{m}) "\right.$, "Força em N" $\left.\}\right]$

ListPlot [\{data4,data2 $\}$,Joined->True,PlotRange->

All,AxesLabel->\{" $\rho_{0}(\mu \mathrm{m})$ ", "Força em N" $\}$,PlotLegends->\{"Força no eixo(x,z) ","Força

radial $\left.\mathrm{x}, \mathrm{y}^{\prime \prime}\right\}$ ]

data5 $=$ Transpose $[\{$ GridX,ForcaParalelo $\}]$;

ListPlot[data5,Joined->True,PlotRange->

All,AxesLabel->\{"$\rho_{0}(\mu \mathrm{m}) "$, "Força em N" $\left.\}\right]$

data6=Transpose $[\{$ GridX,ForcaPerpendicular $\}$;

ListPlot[data6,Joined-> True,PlotRange- $>$ All,AxesLabel->

"$\rho_{0}(\mu \mathrm{m}) "$, "Força em N"\}]

Forçatotal5 $=\operatorname{Sqrt}\left[(\operatorname{Re}[\text { ForcaParalelo }])^{\wedge} 2+\left(\operatorname{Re}[\text { ForcaZParalelo }]^{\wedge} 2\right)\right]$;

data7 $=$ Transpose $[\{$ GridX,Forçatota15 $\}]$;

ListPlot[data7,Joined->True,PlotRange->All,AxesLabel-> 


\section{Apêndice F}

Programa para processamentos das imagens das microesferas em movimento Browniano para obtenção da velocidade quadrática media

1 Importação das imagens e aplicação da função de mudança de cores

SetDirectory[NotebookDirectory[]]

li=Import["nome do vídeo1 .avi",\{"Frames",Range[Frames(inicial),Frames(final)]\}];

$\operatorname{li}[[1]]$

reduce[image $]:=$ Module $[\{\mathrm{t} 1, \mathrm{t} 2\}$, $\mathrm{t} 1=$ Dilation[image,DiskMatrix[i]];

$\mathrm{t} 2=$ Sharpen $[\mathrm{t} 1,3]$;

ColorNegate[t2]

]

Nessa parte do programa, para cada vídeo, nós definimos o diretório onde o vídeo deveria estar salvo e, com o programa, o importamos para o software, representado pela linha 2, onde o comando Range[Frames(inicial),Frames (final)] seleciona os frames que serão importados. Esses frames são determinamos após assistirmos aos vídeos e selecionarmos os trechos que achamos adequados. A taxa de captura é conhecida nessa etapa. Na linha [3], selecionamos o frame inicial e medimos o diâmetro da bead em pixels com o editor de imagem do "Mathematica", e, sabendo o diâmetro das microesferas sabemos a proporção em pixels. A linha 4 faz com que os pixels em branco sejam expandidos em forma de esfera e o sherpen delimita as áreas sem uma cor definida. O Colornegate inverte as cores de branco para preto, onde i é o número que mudamos até o que a bead torne-se uma esfera de tamanho similar à bead do primeiro frame. Vale salientar que as microesferas deve estar no mesmo foco durante o intervalo analisado. 
reduce[12[[1]]]

resulti=reduce/@li;

For $[\mathrm{n}=1, \mathrm{n}<=$ Length[resulti], $\mathrm{n}++$,

Export[StringJoin["novo1_00",ToString[n],.’bmp"],resulti[[n]] ]]

Na linha [6] aplicamos a função da linha [4] a todos os Frames e, na linha [7], exportamos os Frames em imagem bmp para o diretório onde estão salvos o programa e os vídeos. 


\section{Apêndice G}

Cálculo da matriz óptica

\section{programa da matriz ótica}

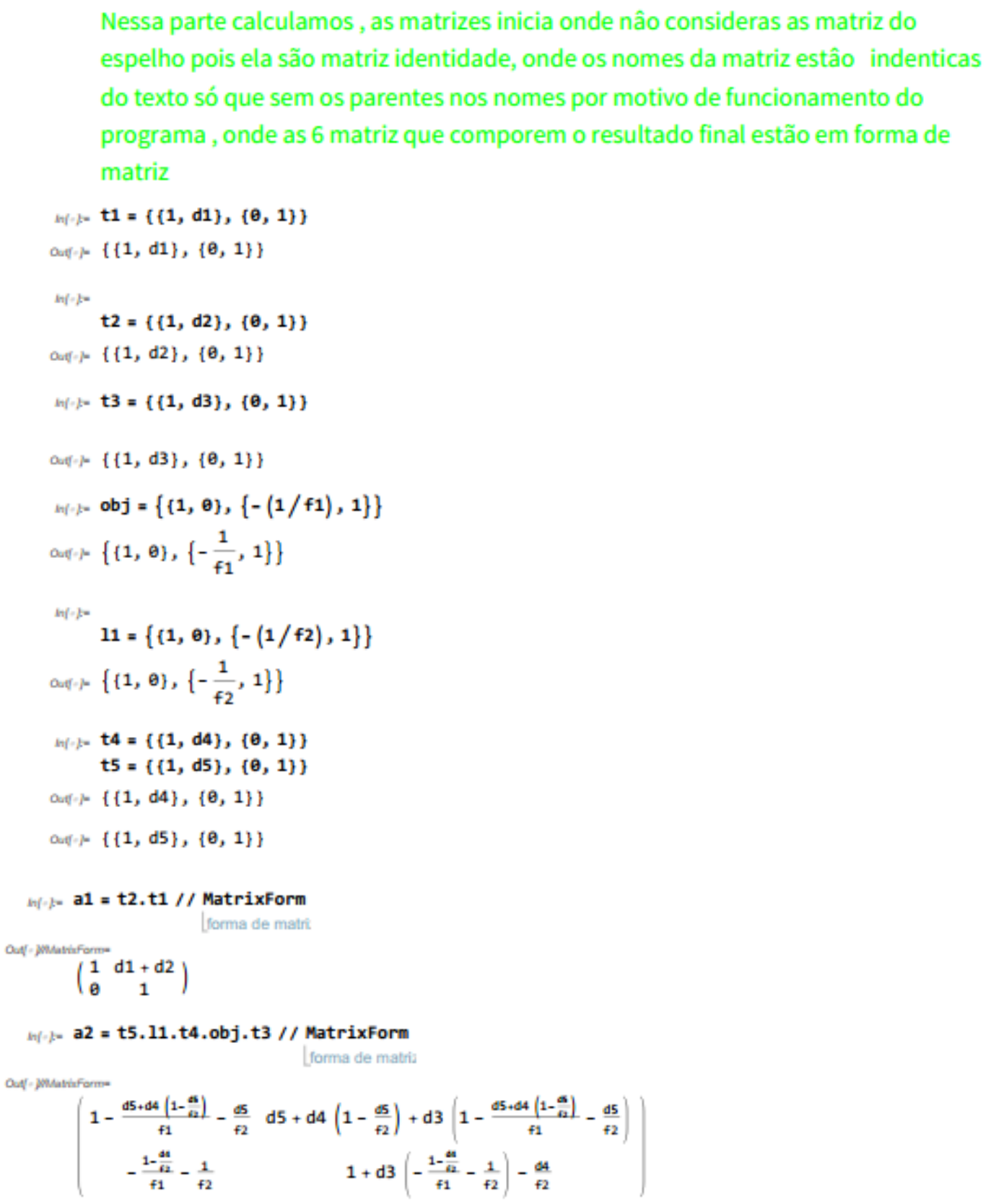




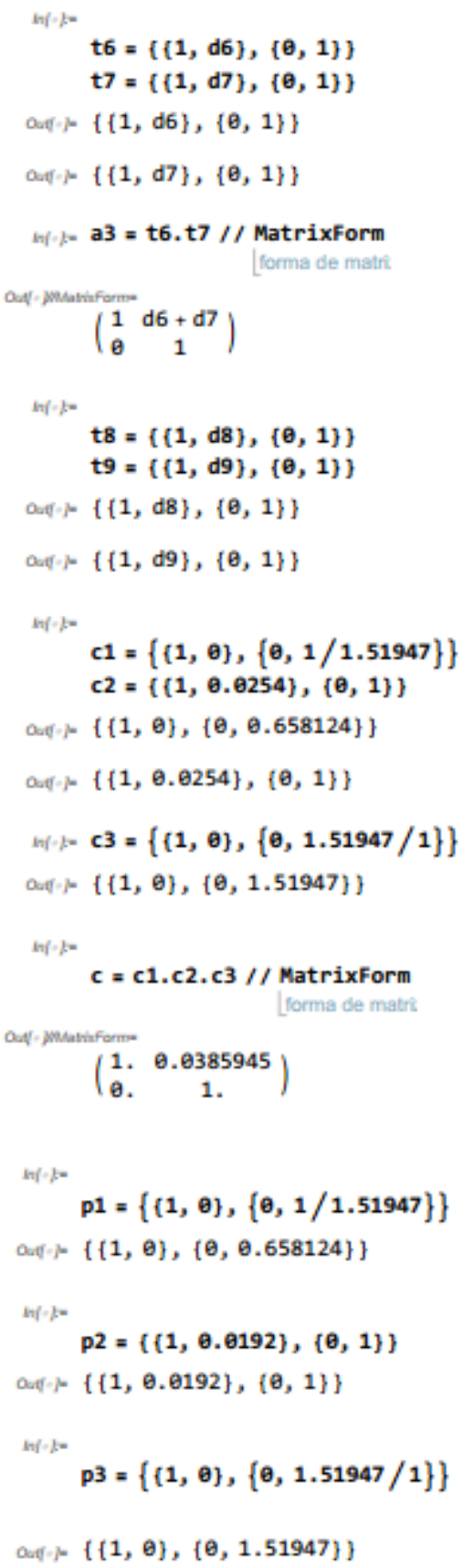




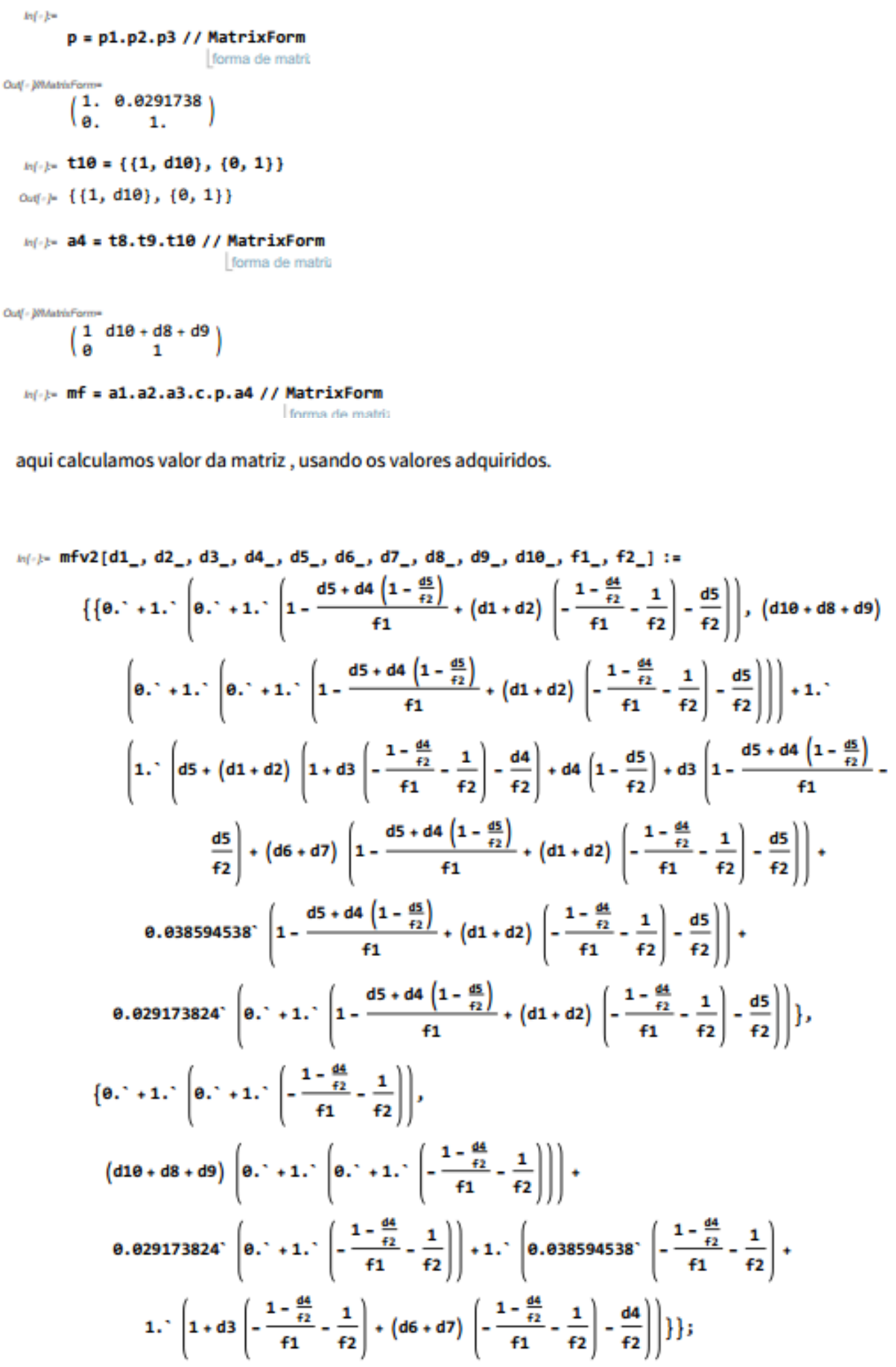

aqui calculamos valor da matriz, usando os valores adquiridos.

$$
\begin{aligned}
& h(n)=m f=m 2\left[d 1_{-}, d 2_{-}, d 3_{-}, d 4_{-}, d 5_{-}, d 6_{-}, d 7_{-}, d 8_{-}, d 9_{-}, d 10_{-}, f 1_{-}, f 2_{-}\right]:= \\
& \left\{\left\{\theta .^{*}+1 .^{v}\left(\theta .^{*}+1 .^{*}\left(1-\frac{d 5+d 4\left(1-\frac{d 5}{f 2}\right)}{f 1}+(d 1+d 2)\left(-\frac{1-\frac{d 4}{f 2}}{f 1}-\frac{1}{f 2}\right)-\frac{d 5}{f 2}\right)\right),(d 10+d 8+d 9)\right.\right.
\end{aligned}
$$

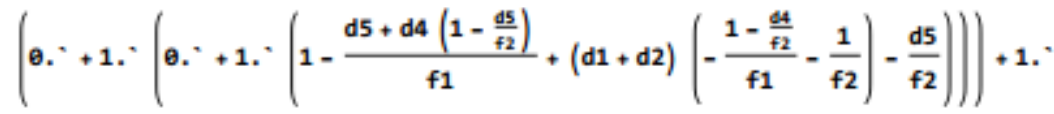

$$
\begin{aligned}
& \left(1 . \cdot\left(d 5+(d 1+d 2)\left(1+d 3\left(-\frac{1-\frac{d 4}{f 2}}{f 1}-\frac{1}{f 2}\right)-\frac{d 4}{f 2}\right)+d 4\left(1-\frac{d 5}{f 2}\right)+d 3\left(1-\frac{d 5+d 4\left(1-\frac{d 5}{f 2}\right)}{f 1}-\right.\right.\right. \\
& \left.\left.\frac{d 5}{f 2}\right)+(d 6+d 7)\left(1-\frac{d 5+d 4\left(1-\frac{d 5}{f 2}\right)}{f 1}+(d 1+d 2)\left(-\frac{1-\frac{d 4}{f 2}}{f 1}-\frac{1}{f 2}\right)-\frac{d 5}{f 2}\right)\right)+ \\
& \left.0.038594538^{\circ}\left(1-\frac{d 5+d 4\left(1-\frac{d 5}{f 2}\right)}{f 1}+(d 1+d 2)\left(-\frac{1-\frac{d 4}{f 2}}{f 1}-\frac{1}{f 2}\right)-\frac{d 5}{f 2}\right)\right)+ \\
& \left.0.029173824^{-}\left(\theta .^{*}+1 .^{\cdot}\left(1-\frac{d 5+d 4\left(1-\frac{d 5}{f 2}\right)}{f 1}+(d 1+d 2)\left(-\frac{1-\frac{d 4}{f 2}}{f 1}-\frac{1}{f 2}\right)-\frac{d 5}{f 2}\right)\right)\right\} \text {, }
\end{aligned}
$$

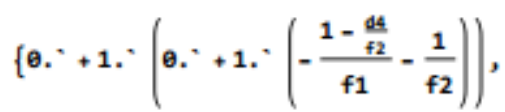

$$
\begin{aligned}
& (d 10+d 8+d 9)\left(\theta .^{*}+1 .^{*}\left(\theta .^{*}+1 .^{*}\left(-\frac{1-\frac{d 4}{f 2}}{f 1}-\frac{1}{f 2}\right)\right)\right)+ \\
& 0.029173824^{\prime}\left(0 . V^{\prime}+1 .^{\prime}\left(-\frac{1-\frac{d 4}{f 2}}{f 1}-\frac{1}{f 2}\right)\right)+1 .^{\prime}\left(0.038594538^{*}\left(-\frac{1-\frac{d 4}{f 2}}{f 1}-\frac{1}{f 2}\right)+\right. \\
& \text { 1. } \left.\left.\left.\left(1+d 3\left(-\frac{1-\frac{d 4}{f 2}}{f 1}-\frac{1}{f 2}\right)+(d 6+d 7)\left(-\frac{1-\frac{d 4}{f 2}}{f 1}-\frac{1}{f 2}\right)-\frac{d 4}{f 2}\right)\right)\right\}\right\} ;
\end{aligned}
$$


